\title{
'Be prepared! : motivational interviewing as pre-treatment in chronic pain rehabilitation
}

Citation for published version (APA):

Mertens, V. C. (2015). 'Be prepared! : motivational interviewing as pre-treatment in chronic pain rehabilitation. [Doctoral Thesis, Maastricht University]. Maastricht University. https://doi.org/10.26481/dis.20151216vm

Document status and date:

Published: 01/01/2015

DOI:

10.26481/dis.20151216vm

Document Version:

Publisher's PDF, also known as Version of record

\section{Please check the document version of this publication:}

- A submitted manuscript is the version of the article upon submission and before peer-review. There can be important differences between the submitted version and the official published version of record.

People interested in the research are advised to contact the author for the final version of the publication, or visit the DOI to the publisher's website.

- The final author version and the galley proof are versions of the publication after peer review.

- The final published version features the final layout of the paper including the volume, issue and page numbers.

Link to publication

\footnotetext{
General rights rights.

- You may freely distribute the URL identifying the publication in the public portal. please follow below link for the End User Agreement:

www.umlib.nl/taverne-license

Take down policy

If you believe that this document breaches copyright please contact us at:

repository@maastrichtuniversity.nl

providing details and we will investigate your claim.
}

Copyright and moral rights for the publications made accessible in the public portal are retained by the authors and/or other copyright owners and it is a condition of accessing publications that users recognise and abide by the legal requirements associated with these

- Users may download and print one copy of any publication from the public portal for the purpose of private study or research.

- You may not further distribute the material or use it for any profit-making activity or commercial gain

If the publication is distributed under the terms of Article $25 \mathrm{fa}$ of the Dutch Copyright Act, indicated by the "Taverne" license above, 


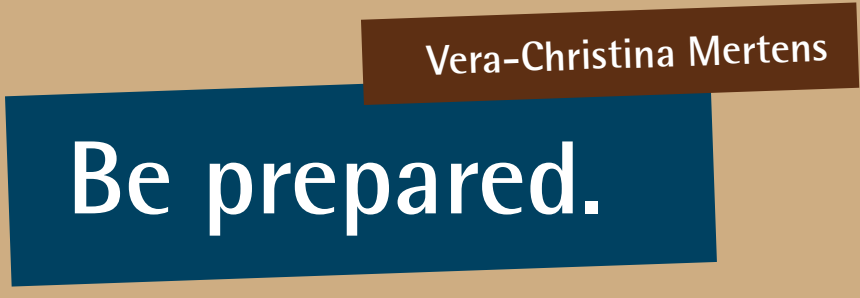

Motivational Interviewing as pre-treatment in chronic pain rehabilitation.

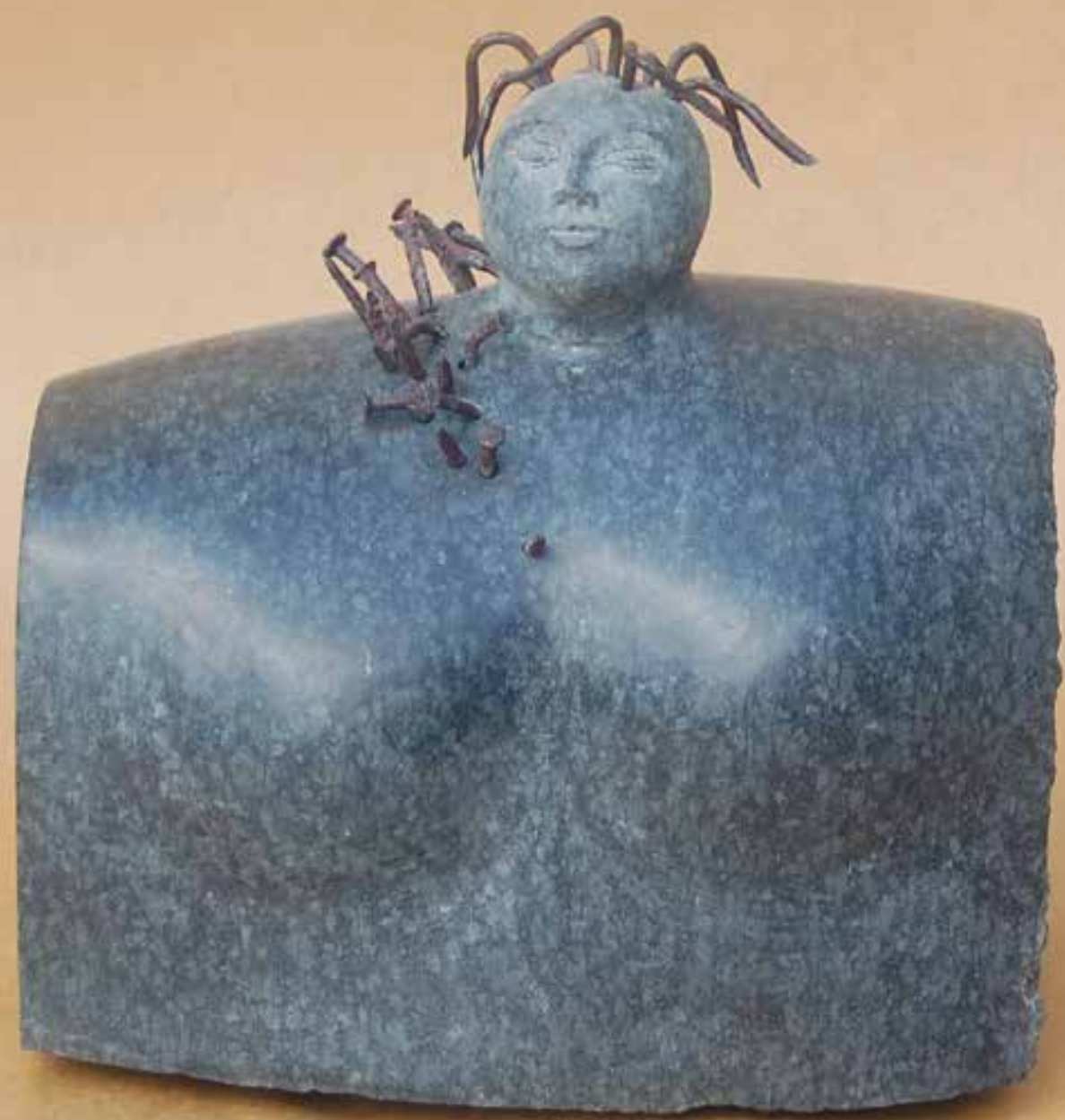


○ Vera-Christina Mertens, Dalhem 2015

ISBN 978-94-6233-114-3

Illustration and Layout: Birgit Blum, www.blum-unternehmenskommunikation.de Skulpturen: Christiane Püttmann, Wuppertal, www.chr-puettmann.de

Fotos: Gregor Rasch

All rights reserved. No part of this thesis may be reproduced or transmitted in any form or by any means, electronic or mechanical, including photocopying, recording or any information storage or retrieval system, without prior written permission of the holder of the copyright. 


\section{Be prepared.}

Motivational Interviewing as

pre-treatment in chronic pain rehabilitation. 
Motivational Interviewing as pre-treatment in chronic pain rehabilitation.

\section{DISSERTATION}

to obtain the degree of Doctor at Maastricht University, on the authority of the Rector Magnificus, Prof Dr. L.L.G. Soete in accordance with the decision of the Board of Deans, to be defended in public on Wednesday, 16 December 2015, at 16.00 hours

by Vera-Christina Mertens born 7th July in Bad Kreuznach, Germany 


\section{PROMOTORES}

Prof. Dr. R.J.E.M. Smeets

Prof. Dr. J.A. Verbunt

\section{CO-PROMOTOR}

Dr. M.E.J.B. Goossens

\section{READING COMIMITTEE}

Prof. Dr. T. van der Weijden (chair)

Dr. M. D. van Eijsden-Besseling

Dr. H. M. van Keulen, TNO Leiden

Prof. Dr. M.W. Post, Rijksuniversiteit Groningen

Prof. Dr. J.W.S. Vlaeyen, Katholieke Universiteit Leuven, Belgium

The research presented in this thesis was conducted at the School for Public Health and Primary Care: CAPHRI, Department of Rehabilitation Medicine of Maastricht University. CAPHRI participates in the Netherlands School of Primary Care Research CaRe.

Financial support by Adelante Rehabilitation Centre (Hoensbroek) and the Dutch Arthritis foundation (reumafonds) for the publication of this thesis is gratefully acknowledged. 


\section{Contents}

\section{CHAPTER 1}

General Introduction

\section{CHAPTER 2}

Effects of nurse-led Motivational Interviewing of patients with chronic musculoskeletal pain in preparation of rehabilitation treatment (PREPARE) on societal participation, attendance level, and cost-effectiveness: study protocol for a randomized controlled trial.

\section{CHAPTER 3}

Treatment fidelity of a nurse-led Motivational Interviewing-based pre-treatment in pain rehabilitation

\section{CHAPTER 4}

How to measure Motivational Interviewing fidelity in randomized controlled trials:

Practical recommendations

\section{CHAPTER 5}

Prepared for treatment? Process evaluation of a nurse-led Motivational Interviewing-based pre-treatment in pain rehabilitation 103

\section{CHAPTER 6}

Prepared for treatment? Effectiveness of a nurse-led Motivational Interviewing based pre-rehabilitation-treatment for patients with non-specific chronic pain:

a randomized controlled trial

\section{CHAPTER 7}

What does the CEO mean to you? Content validity of the Credibility and Expectancy Questionnaire (CEO) in a pain rehabilitation setting

\section{CHAPTER 8}

General Discussion

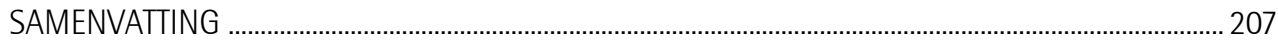

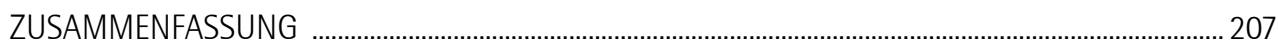

ACKNOWLEDGEMENTS - DANKWOORD - DANKWORT ………........................................................ 217

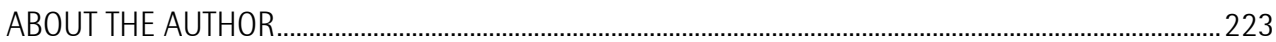


Der Mensch sollte alle seine Werke zunächst einmal in seinem Herzen erwägen, bevor er sie ausführt.

Hildegard von Bingen (1098 - 1179)

für Pütti 


\section{Chapter 1}

General Introduction

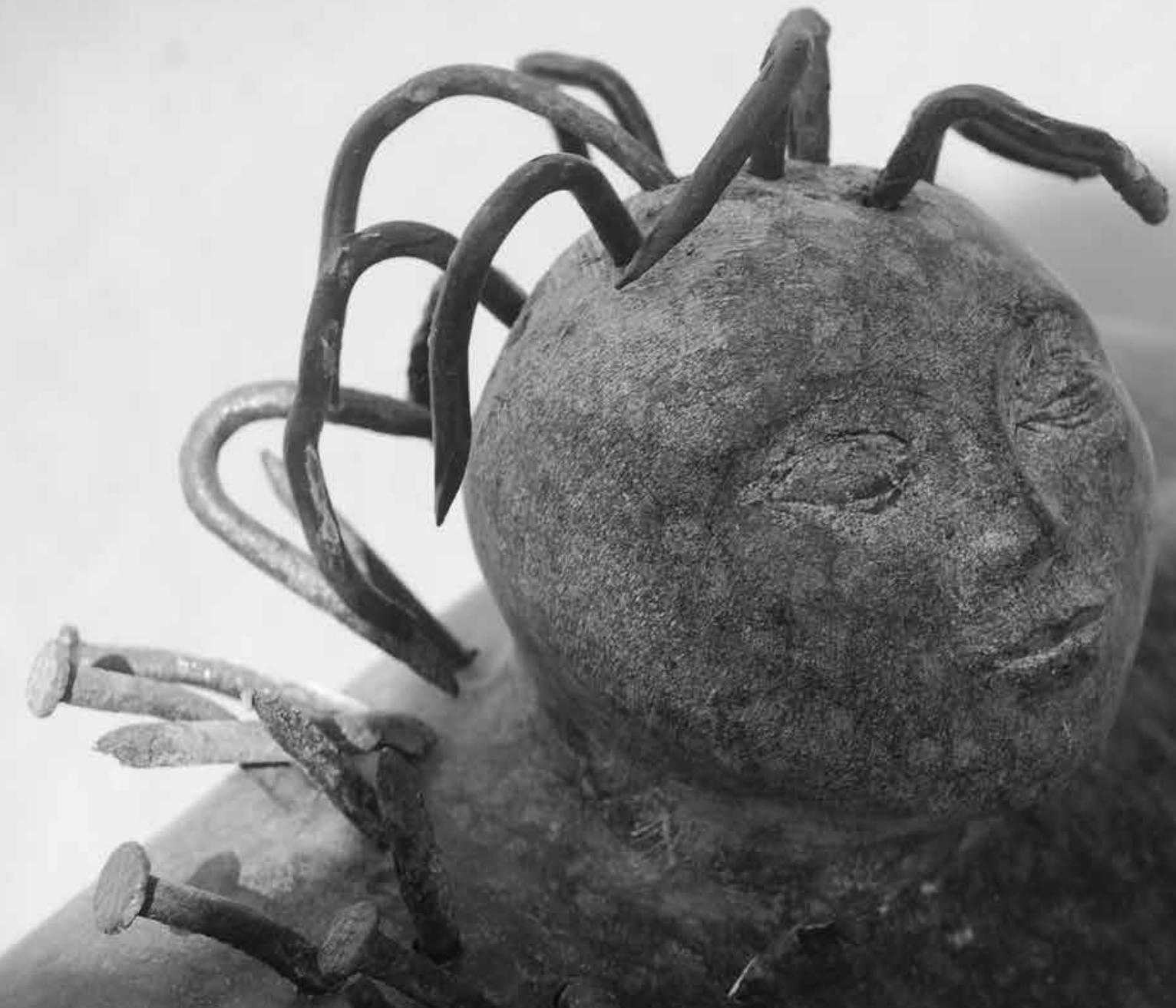




\section{General Introduction}

Chronic musculoskeletal pain is a distressing health condition affecting a person's life and the social surrounding.

This thesis presents the results of the research project 'Pre-treatment Pain Rehabilitation' (Prepare). Therein, a pre-treatment was developed and evaluated by means of a randomized controlled trial and the role of expectancy was investigated.

\section{Chronic pain: Epidemiology and the consequences}

The International Association for the Study of Pain (IASP) defines pain as "an unpleasant sensory or emotional experience associated with actual or potential tissue damage, or described in terms of such damage" and chronic pain is defined as "pain which persists beyond normal tissue damage, or described in terms of such damage" [40].

Chronic musculoskeletal pain (referring to pain existing longer than six months) occurs frequently; circa 19\% of the adult population in Europa is suffering from chronic musculoskeletal pain [10]. Most cases of chronic pain can be categorized as non-specific, referring to no known underlying pathology. Only the small percentage of 5-10\% of all cases can be considered as pain of specific nature such as degenerative, neurological or inflammatory conditions (e.g. rheumatoid arthritis). For low back pain symptoms, this is the case even in the light of new imaging techniques [17]. Examples of non-specific chronic pain are syndromes such as complex regional pain syndrome (CRPS), low back pain, and Complaints of arm, neck, and/or shoulder (CANS).

A particular form of chronic pain is fibromyalgia, characterized by widespread pain, fatigue, unrefreshing sleep and cognitive problems [61]. It is often categorized as centralized pain state [15] referring to pain in different body regions at different times [65]. Its prevalence seems to be independent of country and culture [32] and varies, depending on diagnostic criteria, between $0.7 \%$ and $8 \%$ of a population $[32,59,63,64]$. Women are suffering from this condition approximately two to three times more often than men $[59,62]$.

At this moment, different opinions exist regarding the naming of the diagnosis. Some suggest to replace fibromyalgia by 'polysymptomatic distress', referring to the most prevalent association in those patients [5]. Whereas others argue it should be labeled as 'somatoform disorder' [23] referring also to the Diagnostic and Statistical Manual of Mental Disorders (5th edition; DSM-5, American Psychiatric Association, 2013) wherein it is stated as 'somatic symptom disorder' (SSD) [1]. Once more others propose the label 'complex widespread pain syndrome' [41]. In this dissertation, we use the term fibromyalgia. 


\section{Consequences}

Chronic musculoskeletal pain has multiple consequences for the patients, their families, and for the society as a whole.

It is known that the mental health consequences for those suffering from chronic non-specific pain can be detrimental leading to a higher level of depression and lower quality of life [2]. Although numerous studies report, a positive relationship between chronic pain and disability [2], chronic pain states have also often been referred to as 'invisible disability'.

In qualitative studies exploring the lived experience of those living with chronic pain, furthermore feelings of disintegration of identity are described [45]. Also the feeling not to be listened to by the medical professional system as well as the social surrounding are mentioned (e.g. [54]), acknowledging chronic pain states as 'invisible disability' [56]. Especially by patients with so called non-specific chronic pain the feeling of not been acknowledged and not heard is frequently expressed [14].

The social life (e.g. social activities, relationships and leisure time) is highly influenced [10,18], and this is also applicable for belonging partners [55].

Chronic pain impacts not only the individual with pain, but also the society: The costs for the society are substantial, and even higher as the costs for heart diseases, cancer, and diabetes [19].

To conclude, chronic non-specific pain is a problem occurring frequently. It has significant implications for those in pain, their social surrounding, and the whole society.

\section{State-of-the-art pain rehabilitation treatment}

As mentioned before, chronic pain affects all aspects of human life. In the Netherlands, most rehabilitation treatments therefore are focusing on reducing the impact of pain on one's level of participation in society. Most programs are behaviorally oriented, taking the biopsychosocial nature of chronic pain into account [28]. By learning patients to cope with their pain, the aim of multidisciplinary pain rehabilitation treatment approaches is to improve functioning and increase social participation and quality of life in the long-term [57].

Meta-analyses have shown moderate effects of multidisciplinary pain rehabilitation treatment in chronic low back pain in terms of a reduction of disability [26]. Also for fibromyalgia the effectiveness of cognitive-behavioral oriented treatments in terms of reducing disability, pain, and negative mood at both the end of the treatment and follow-up was shown [7].

The international Classification of Functioning, Disability, and Health (ICF) of the World Health Organization (WHO) is the basis of rehabilitation treatment. The ICF is a holistic concept containing bio-psycho-social aspects of health and well-being [39].

The ICF can serve as conceptual model to define functioning and disability. Participation is therein introduced, and defined as 'involvement in life situations' [39] and serves as important outcome measure for rehabilitation.

\section{Room for improvement in the effectiveness of current pain rehabilitation treatment}

Despite the proven effectiveness of current multidisciplinary pain rehabilitation treatments, high numbers of low adherence and drop-out are reported. The percentage of non-adherence to pain 
rehabilitation ranges from $9 \%$ to $42 \%[6,42,46]$. As most patients with chronic pain have previously undergone several treatments aiming for pain reduction, the initial expectation of most patients is that their pain will also be reduced during the rehabilitation treatment $[43,58]$. This often unresolved discrepancy between the expectations of the patient and the treatment approach may result in demotivation, lack of trust in therapy, non-adherence, and subsequently drop out of pain rehabilitation treatment $[51,52]$. Therefore, achieving sufficiently high treatment credibility and creating right expectations are a big challenge. It is known that higher levels of treatment credibility and expectancy are positively associated with the outcome of treatment. Those concepts are predominantly investigated in the field of psychotherapy research and in the field of complementary medicine (e.g. [44]), but recently some work has also been done in the field of acupuncture and the treatment of musculoskeletal pain $[8,50]$. In the field of pain rehabilitation treatments, the predictive value [53] and moderating role [20] of expectancy on treatment outcome was confirmed.

Several instruments exist to measure expectancy [21]. At this moment there is no gold standard available, and not all aspects of validity of all instruments have been extensively investigated. A frequently used instrument is the Credibility and Expectancy Questionnaire (CEO) [16].

\section{Motivational Interviewing (MI)}

\section{What is MI? (Definition)}

As remedy for motivation and adherence problems, Motivational Interviewing (MI) emerged first in counseling in the field of alcohol addiction [33]. Miller's concepts of motivation were ground breaking in the field of addiction treatment at that time [9] and came along with the fact that $\mathrm{MI}$ coincided with a paradigm shift in the field of addiction treatment [24]. Interestingly, MI got popular before evidence of its efficacy was shown [9].

$\mathrm{MI}$ is defined as a person-centered form of counseling to elicit and strengthen motivation for change [35]. Ml's purpose is to explore and strengthen the inner motivation (intrinsic motivation) within a person in order to enhance behavioral change [48].

\section{Evidence for Motivational Interviewing}

MI became quite popular in health care indicated by almost exponentially rising numbers of trials during the last twenty years. At this moment, several meta analyses are available wherein $\mathrm{Ml}$ has shown to be an effective treatment compared to other active treatments such as cognitive behavior therapy (CBT) in a wide range of behaviors (e.g. physical activity, smoking cessation) and contexts (e.g. primary care, tertiary prevention) in health care $[11,25,30,49]$. MI has shown to be especially effective as pre-treatment prior to the main treatment $[12,25,29]$.

In the field of musculoskeletal health care, first studies on the topic of MI have just recently been published. MI is mostly used as mean to improve adherence to treatment or adherence to physical activity. Habib et al. (2005) aimed to prepare patients with chronic pain for subsequent CBT-oriented pain management workshops by means of two MI-based counseling sessions provided by a 
psychologist and compared its effectiveness with two attention control sessions. Therein, positive results in terms of subsequent attendance in pain management workshops favoring the $\mathrm{Ml}$ intervention were found [22]. Vong et al. (2011) used Motivational Enhancement Therapy, a subtype of $\mathrm{MI}$, in outpatient physiotherapy care for patients with chronic pain and compared its effectiveness with physical therapy [60]. Positive results in terms of improved physical functioning and exercise compliance, and trends in reduction of pain intensity and functional disability were shown [60]. In a telephone-delivered RCT the results of MI were compared with an education control group aiming on an increase of physical activity and the improvement of pain-related impairments in patients with fibromyalgia [3,4]. The primary outcomes yielded no group differences, but beneficial short-term self-reported physical activity and clinically significant increased walking distance in the Ml-group was found [4].

Furthermore, MI was used in another RCT in patients with rheumatoid arthritis in order to increase their physical activity. Therein, a group-based educational session was compared with an intervention consisting of a group session, a motivational interview by a physical therapist and additionally two nurse-led sessions focusing on self-regulation compared to a group session only. In the intervention group this resulted in increased leisure time, significantly more patients met the recommendation for the amount of physical activity per week, and showed a higher level of autonomous motivation, and self-efficacy to be physically active. Disease activity and functional status were not affected [27].

Although first results seem of interest, the number of studies is still restricted. A systematic review regarding $\mathrm{MI}$ in the field of musculoskeletal care mentioned the need for well-designed randomized controlled trials in this field of study [13].

\section{Elements of MI}

MI consists of relational and technical aspects [37]. The relational aspect refers to the so-called 'MI-spirit', a counselor-attitude characterized by genuine interest in the client and empathy. The technical aspect consists of techniques to evocate, elicit, and reinforce change talk [47] (by e.g. the use of open questions and reflections). These technical aspects help to realize the relational aspects of $\mathrm{MI}$, which are more difficult to learn and maintain than the technical aspects. The underlying principles resulting in MI-spirit like empathy are a complex mix of skills that take considerable time to learn [35].

However, so far, MI has mostly been emphasized as a spirit rather than a technique. "MI is simple but not easy" wrote Miller and Rollnick and summarized mixed blessing challenges regarding MI [35]. Four key processes are described in the third version of MI [36]. They emerge in the order described and contain: (1) engaging with a client, (2) focusing on an agreeable direction, (3) evoking ambivalence by questions and reflections, and (4) planning the change. Within this description, MI can be seen as flow of processes which "flow into each other, overlap, and recur" ([36], p. 26).

To ensure this rather flaw flow of Ml's elements and to ensure treatment fidelity within effectiveness studies, coding systems instead of MI manuals have been established within the MI community [9]. The first established was the Motivational Interviewing Skills Code (MISC) [34]. Several 
other instruments have been developed till now [31]. One less comprehensive alternative to the MISC is the Motivational Interviewing Treatment Integrity (MITI) code [38].

\section{Aims and outline of this dissertation}

The previous section has shown that pain rehabilitation is moderately effective for several non-specific chronic pain states but suffers at the same time from non-adherence and drop-out from treatment. Improving adherence and reducing drop out thus seem important targets in order to improve the effectiveness of pain rehabilitation treatment.

Our aim is the evaluation of the effectiveness and applicability of $\mathrm{MI}$ as a pre-treatment. $\mathrm{MI}$ seems particularly promising in improving motivation for and adherence to treatment in order to prevent drop-out from pain rehabilitation treatment.

This dissertation consists of six subsequent chapters in which different aspects of MI as pre-treatment and the role of expectancy are discussed.

Chapter 2 outlines the design and rationale of the Prepare randomized controlled trial. Therein, all measurement instruments chosen and sub studies are introduced as well as rationale and summary of the intervention is given.

Chapter 3 presents the results of the Ml-fidelity check within the Prepare randomized controlled trial. Therein, sessions of the control as well as the intervention arm were evaluated by using the Motivational Interviewing Treatment Integrity Instrument (MITI).

Chapter $\mathbf{4}$ gives practical recommendations regarding the fidelity evaluation of studies using Motivational Interviewing (MI).

Chapter $\mathbf{5}$ describes the results of the process evaluation on the feasibility of the intervention arm of the Prepare trial which was performed prior to knowing the effects of the intervention.

Chapter 6 reports upon the long-term results of the nurse-led Prepare randomized controlled trial, which compared pain pre-treatment education to MI pre-treatment. This study examined the effects in terms of social participation, pain-related functioning, and drop-out from treatment. Additionally, predefined moderating factors were investigated.

Chapter 7 provides insights in the content validity of a questionnaire measuring treatment credibility and expectancy, the Credibility and Expectancy Questionnaires (CEO).

To conclude, in Chapter 8 a summary of the main findings is given, methodological limitations and implications for clinical practice are discussed, and implications for future research in the context of chronic pain, pain rehabilitation, and Motivational Interviewing are presented. 


\section{References}

[1] American Psychiatric Association, Diagnostic and statistical manual of mental disorders:

DSM-5, 2013.

[2] Andersen LN, Kohberg M, Juul-Kristensen B, Herborg LG, Søgaard K, Roessler KK. Psychosocial aspects of everyday life with chronic musculoskeletal pain: A systematic review. Scand J Pain 2014;5(2):131-148.

[3] Ang DC, Kaleth AS, Bigatti S, Mazzuca S, Saha C, Hilligoss J, Lengerich M, Bandy R. Research to Encourage Exercise for Fibromyalgia (REEF): Use of motivational interviewing design and method. Contemp Clin Trials 2010;32(1):59-68.

[4] Ang DC, Kaleth AS, Bigatti S, Mazzuca SA, Jensen MP, Hilligoss J, Slaven J, Saha C. Research to encourage exercise for fibromyalgia (REEF): use of motivational interviewing, outcomes from a randomized-controlled trial. Clin J Pain 2013;29(4):296-304.

[5] Bass C, Henderson M. Fibromyalgia: an unhelpful diagnosis for patients and doctors. BMJ 2014;348:g2168.

[6] Bendix AE, Bendix T, Haestrup C, Busch E. A prospective, randomized 5-year follow-up study of functional restoration in chronic low back pain patients. Eur Spine J 1998;7(2):111-119.

[7] Bernardy K, Klose P, Busch AJ, Choy EH, Hauser W. Cognitive behavioural therapies for fibromyalgia. Cochrane Database Syst Rev 2013;9:CD009796.

[8] Bialosky JE, Bishop MD, Cleland JA. Individual expectation: an overlooked, but pertinent, factor in the treatment of individuals experiencing musculoskeletal pain. Phys Ther 2010;90(9):1345-1355.

[9] Björk A. Stabilizing a fluid intervention: The development of Motivational Interviewing, 1983-2013. Addict Res Theory 2014;22(4):313-324.

[10] Breivik H, Collett B, Ventafridda V, Cohen R, Gallacher D. Survey of chronic pain in Europe: prevalence, impact on daily life, and treatment. Eur J Pain 2006;10(4):287-333. 
[11] Burke B, Arkowitz H, Menchola M. The efficacy of motivational interviewing: a meta-analysis of controlled clinical trials. J Consult Clin Psychol 2003;71(5):843-861.

[12] Burke BL, Arkowitz $H$, Menchola M. The efficacy of motivational interviewing: a meta-anaIysis of controlled clinical trials. J Consult Clin Psychol 2003;71(5):843-861.

[13] Chilton R, Pires Yfantouda R, Wylie M. A systematic review of motivational interviewing within musculoskeletal health. Psychol Health Med 2012;17(4):392-407.

[14] Clarke A, Martin D, Jones D, Schofield P, Anthony G, McNamee P, Gray D, Smith BH. "I Try and Smile, I Try and Be Cheery, I Try Not to Be Pushy. I Try to Say 'I'm Here for Help' but I Leave Feeling... Worried": A Qualitative Study of Perceptions of Interactions with Health Professionals by Community-Based Older Adults with Chronic Pain. PLoS One 2014;9(9):e105450.

[15] Clauw DJ. Fibromyalgia: a clinical review. JAMA 2014;311(15):1547-1555.

[16] Devilly GJ, Borkovec TD. Psychometric properties of the credibility/expectancy questionnaire. J Behav Ther Exp Psychiatry 2000;31(2):73-86.

[17] Deyo RA, Weinstein JN. Low back pain. N Engl J Med 2001;344(5):363-370.

[18] Froud R, Patterson S, Eldridge $S$, Seale C, Pincus T, Rajendran D, Fossum C, Underwood M. A systematic review and meta-synthesis of the impact of low back pain on people's lives. BMC Musculoskelet Disord 2014;15:50.

[19] Gaskin DJ, Richard P. The economic costs of pain in the United States. J Pain 2012;13(8):715-724.

[20] Goossens ME, Vlaeyen JW, Hidding A, Kole-Snijders A, Evers SM.

Treatment expectancy affects the outcome of cognitive-behavioral interventions in chronic pain. Clin J Pain 2005;21(1):18-26; discussion 69-72.

[21] Haanstra TM, van den Berg T, Ostelo RW, Poolman RW, Jansma EP, Cuijpers P, de Vet HC. Systematic review: do patient expectations influence treatment outcomes in total knee and total hip arthroplasty? Health Qual Life Outcomes 2012;10:152.

[22] Habib S, Morrissey S, Helmes E. Preparing for pain management: a pilot study to enhance engagement. J Pain 2005;6(1):48-54. 
[23] Häuser W, Burgmer M, Kollner V, Schaeferts R, Eich W, Hausteiner-Wiehle C, Henningsen P. Fibromyalgia syndrome as a psychosomatic disorder - Diagnosis and therapy according to current evidence-based guidelines. Z Psychosom Med Psychother 2013;59(2):132-152.

[24] Heather N. Motivational interviewing: Is it all our clients need?

Addict Res Theory 2005;13(1):1-18.

[25] Hettema J, Steele J, Miller WR. Motivational interviewing.

Annu Rev Clin Psychol 2005;1:91-111.

[26] Kamper SJ, Apeldoorn AT, Chiarotto A, Smeets RJ, Ostelo RW, Guzman J, Van Tulder MW. Multidisciplinary biopsychosocial rehabilitation for chronic low back pain.

Cochrane Database Syst Rev 2014;9:CD000963.

[27] Knittle K, De Gucht V, Hurkmans E, Peeters A, Ronday K, Maes S, Vlieland TV.

Targeting motivation and self-regulation to increase physical activity among patients with rheumatoid arthritis: a randomised controlled trial. Clin Rheumatol 2013;34(2):231-238.

[28] Köke A, Brouwers M, Heuts P, Schiphorst Preuper R, Smeets R, Swaan L, Vlaeyen J, Patijn J. Consensus

Rapport Pijnrevalidatie Nederland. Een beschrijvend overzicht van pijnrevalidatieprogramma's in Nederland met betrekking tot doelen en inhoud. [Consensus Report on pain rehabilitation in the Netherlands. A descriptive overview of pain rehabilitaion programs in the Netherlands as regards goals and content].

Maastricht: PijnKennis Centrum Maastricht [Pain Knowledge Centre Maastricht], 2005.

[29] Lundahl B, Burke BL. The effectiveness and applicability of motivational interviewing: A practice-friendly review of four meta-analyses. J Clin Psychol 2009;65(11):1232-1245.

[30] Lundahl B, Moleni T, Burke BL, Butters R, Tollefson D, Butler C, Rollnick S.

Motivational interviewing in medical care settings: a systematic review and meta-analysis of randomized controlled trials. Patient Educ Couns 2013;93(2):157-168.

[31] Madson MB, Campbell TC. Measures of fidelity in motivational enhancement: a systematic review. J Subst Abuse Treat 2006;31(1):67-73.

[32] McBeth J, Jones K. Epidemiology of chronic musculoskeletal pain.

Best Pract Res Clin Rheumatol 2007;21(3):403-425.

[33] Miller WR. Motivational interviewing with problem drinkers.

Behav Psychother 1983;11:147-172. 
[34] Miller WR, Mount KA. A small study of training in Motivational Interviewing: does one workshop change clinician and client behavior?

Behav Cogn Psychoth 2001;29(04):457-471.

[35] Miller WR, Rollnick S. Ten things that motivational interviewing is not.

Behav and Cogn Psychoth 2009;37(2):129-140.

[36] Miller WR, Rollnick S. Motivational interviewing: Helping people change.

New York: The Guilford Press, 2013.

[37] Miller WR, Rose GS. Toward a theory of motivational interviewing.

Am Psychol 2009;64(6):527-537.

[38] Moyers TB, Martin T, Manuel JK, Miller WR, Ernst D. Revised Global Scales: Motivational Interviewing Treatment Integrity 3.1.1 (MITI 3.1.1). In: CoA University of New Mexico, Substance Abuse and Addictions (CASAA) editor, 2010.

[39] Organization WH. International Classification of Functioning, Disability and Health (ICF). 2001.

[40] PAIN IAftSo. IASP taxonomy, 2012.

[41] Perrot S. What can fibromyalgia teach us about chronic pain? An emblematic condition to discuss mind and body concepts, predictive medicine and assessment of chronic condition management. Eur J Pain 2014;18(8):1049-1051.

[42] Peters J, Large RG, Elkind G. Follow-up results from a randomised controlled trial evaluating in- and outpatient pain management programmes. Pain 1992;50(1):41-50.

[43] Petrie KJ, Frampton T, Large RG, Moss-Morris R, Johnson M, Meechan G. What do patients expect from their first visit to a pain clinic? Clin J Pain 2005;21(4):297-301.

[44] Prady SL, Burch J, Vanderbloemen L, Crouch S, MacPherson H. Measuring expectations of benefit from treatment in acupuncture trials: A systematic review.

Complement Ther Med 2015;23(2):185-199.

[45] Råheim M, Haland W. Lived experience of chronic pain and fibromyalgia: women's stories from daily life. Qual Health Res 2006;16(6):741-761.

[46] Rainville J, Ahern DK, Phalen L. Altering beliefs about pain and impairment in a functionally oriented treatment program for chronic low back pain. Clin J Pain 1993;9(3):196-201. 
[47] Rollnick S, Butler CC, Kinnersley P, Gregory J, Mash B. Motivational interviewing BMJ 2010;340.

[48] Rollnick S, Miller WR, Butler C. Motivational Interviewing for Health Care Professionals. New York, NY, USA: Guilford Press, 2007.

[49] Rubak S, Sandbaek A, Lauritzen T, Christensen B. Motivational interviewing: a systematic review and meta-analysis. Br J Gen Pract 2005;55(513):305-312.

[50] Sherman KJ, Cherkin DC, Ichikawa L, Avins AL, Delaney K, Barlow WE, Khalsa PS, Deyo RA. Treatment expectations and preferences as predictors of outcome of acupuncture for chronic back pain. Spine (Phila Pa 1976) 2010;35(15):1471-1477.

[51] Sloots M, Dekker JH, Pont M, Bartels EA, Geertzen JH, Dekker J. Reasons of drop-out from rehabilitation in patients of Turkish and Moroccan origin with chronic low back pain in The Netherlands: a qualitative study. J Rehabil Med 2010;42(6):566-573.

[52] Sloots M, Scheppers EF, Van de Weg FB, Dekker JH, Bartels EA, Geertzen JH, Dekker J. Higher dropout rate in non-native patients than in native patients in rehabilitation in The Netherlands. Int J Rehabil Res 2009;32(3):232-237.

[53] Smeets RJ, Beelen S, Goossens ME, Schouten EG, Knottnerus JA, Vlaeyen JW. Treatment expectancy and credibility are associated with the outcome of both physical and cognitive-behavioral treatment in chronic low back pain.

Clin J Pain 2008;24(4):305-315.

[54] Söderberg S, Lundman B, Norberg A. Struggling for dignity: the meaning of women's experiences of living with fibromyalgia. Qual Health Res 1999;9(5):575-587.

[55] Söderberg S, Strand M, Haapala M, Lundman B. Living with a woman with fibromyalgia from the perspective of the husband. J Adv Nurs 2003;42(2):143-150.

[56] Sturge-Jacobs M. The experience of living with fibromyalgia: confronting an invisible disability. Res Theory Nurs Pract 2002;16(1):19-31.

[57] Turk DC, Wilson HD, Cahana A. Treatment of chronic non-cancer pain. Lancet 2011;377(9784):2226-2235.

[58] Verbeek J, Sengers MJ, Riemens L, Haafkens J. Patient expectations of treatment for back pain: a systematic review of qualitative and quantitative studies. Spine (Phila Pa 1976) 2004;29(20):2309-2318. 
[59] Vincent A, Lahr BD, Wolfe F, Clauw DJ, Whipple MO, Oh TH, Barton DL, St Sauver J. Prevalence of fibromyalgia: a population-based study in Olmsted County, Minnesota, utilizing the Rochester Epidemiology Project.

Arthritis Care Res (Hoboken) 2013;65(5):786-792.

[60] Vong SK, Cheing GL, Chan F, So EM, Chan CC. Motivational enhancement therapy in addition to physical therapy improves motivational factors and treatment outcomes in people with low back pain: a randomized controlled trial. Arch Phys Med Rehabil 2011;92(2):176-183.

[61] Wolfe F, Clauw DJ, Fitzcharles MA, Goldenberg DL, Hauser W, Katz RS, Mease P, Russell AS, Russell IJ, Winfield JB. Fibromyalgia criteria and severity scales for clinical and epidemiological studies: a modification of the ACR Preliminary Diagnostic Criteria for Fibromyalgia. J Rheumatol 2011;38(6):1113-1122.

[62] Wolfe F, Ross K, Anderson J, Russell IJ. Aspects of fibromyalgia in the general population: sex, pain threshold, and fibromyalgia symptoms. J Rheumatol 1995;22(1):151-156.

[63] Wolfe F, Ross K, Anderson J, Russell IJ, Hebert L. The prevalence and characteristics of fibromyalgia in the general population. Arthritis Rheum 1995;38(1):19-28.

[64] Wolfe F, Walitt BT, Hauser W. What is fibromyalgia, how is it diagnosed, and what does it really mean? Arthritis Care Res (Hoboken) 2014;66(7):969-971.

[65] Woolf CJ. Central sensitization: implications for the diagnosis and treatment of pain. Pain 2011;152(3 Suppl):S2-15. 


\section{Chapter 2}

Effects of nurse-led Motivational Interviewing of patients with chronic musculoskeletal pain in preparation of rehabilitation treatment (PREPARE) on societal participation, attendance level, and cost-effectiveness: study protocol for a randomized controlled trial.

Mertens, V.-C.

Goossens, M.E.

Verbunt, J.A.

Köke, A.J.

Smeets, R.J.

Trials. 2013 Apr 2; p.14:90.

DOI: 10.1186/1745-6215-14-90. 


\section{Abstract}

\section{Background}

Non-adherence and drop-out are major problems in pain rehabilitation. For patients with various health problems, Motivational Interviewing (MI) has have shown promising effects to tackle these problems. In chronic pain patients the effectiveness of MI is however unknown. Therefore, a MIbased pre-pain rehabilitation intervention (MIP) addressing motivation, expectations and beliefs has been developed to prepare eligible patients for rehabilitation treatment.

\section{Methods/ design}

Study design: A parallel RCT including two interventions: a Motivational Interviewing pre-pain rehabilitation intervention (MIP) and a usual care (UC) control arm. Follow-up will be six months after completion of rehabilitation treatment.

Study population: 160 ( $n=80$ per arm) patients with chronic non-specific musculoskeletal pain visiting an outpatient rehabilitation department, who are eligible to participate in an outpatient cognitive behavioral pain rehabilitation program.

Intervention: MIP consists of 2 sessions to prepare and motivate the patient for pain rehabilitation treatment and its bio psychosocial approach. UC consists of information and education about the etiology and the general rehabilitation approach of chronic pain. Both the MIP and UC contain two sessions of 45 to 60 minutes, each.

Objective: The aim of the current study is to evaluate the effectiveness of MIP compared to usual care (UC) in terms of an increase in the long-term level of societal participation and decrease of drop-out during treatment of patients with chronic pain receiving rehabilitation treatment.

Main study endpoints: Primary outcome is the change in level of participation (according to the ICF-definition: "involvement in a life situation") six months after completion of rehabilitation treatment. Secondary outcomes are adherence and treatment drop-out, disability, pain intensity, self-reported main complaints, (pain-specific) self-efficacy, motivation, and quality of life. Costs are calculated including the costs of the pre-treatment intervention, productivity losses and health care utilization. Potential moderators and active ingredients of $\mathrm{MI}$ are explored. For the process evaluation, parameters such as MI-fidelity, feasibility, and experiences are explored. 


\section{Discussion}

The results of this study will provide evidence on the effectiveness of this Ml-based pretreatment in pain rehabilitation. Furthermore, a cost-effectiveness analysis and exploration of moderating and working mechanisms of $\mathrm{MI}$ and an extensive process evaluation takes place.

Trial registration: Nederlands Trial Register NTR3065

\section{Background}

Chronic non-specific musculoskeletal pain is a major health burden. It occurs in approximately $10 \%$ of the general population [1] and causes disability [2], medical expenses [3], and a high amount of work absenteeism [4].

In the Netherlands, cognitive behavioural therapy (CBT)-based approaches are part of usual rehabilitation care for patients with non-specific chronic pain [5], and also recommended in the Dutch guidelines [6]. The common assumption of CBT in rehabilitation is that pain and disability are not only influenced by biomedical factors but also by psychological and social factors, which is referred to as the bio psychosocial approach [7]. The primary aim of rehabilitation treatment is to increase the patient's ability to cope with pain instead of curing pain. The ultimate intention is to increase the patient's level of participation in society and his/her quality of life [8]. The ultimate goal and key outcome of rehabilitation is therefore societal participation. According the (International Classification of Functioning, Disability and Health) ICF, participation is therein defined as "involvement in a life situation" whereas the opposite, participation restrictions, are defined as "problems an individual may experience in involvement in life situations" [9].

The Initiative on Methods, Measurements, and Pain Assessment in Clinical Trials (IMMPACT) does not mention the measurement of participation [10]. But in the field of rehabilitation, participation is seen as an important concept in order to reflect meaningful patient-centred outcomes [11]. It is suggested that those outcomes should be included more often in trials [12].

Reviews have shown a moderate effectiveness of cognitive behavioral treatments in chronic non-specific musculoskeletal pain [13] and low back pain [14]. Behavioural therapy is more effective than usual care in terms of pain relief [15], and similar effects are shown for functioning after an intensive multidisciplinary bio-psycho-social rehabilitation program [16].

Unfortunately, in the current rehabilitation care, non-adherence and drop-out are major problems. Adherence rates are low in patients with chronic conditions [17], and subsequently, dropout of pain rehabilitation programmes ranging from $9-42 \%$, is high [18-20]. Previous research showed that adherence and non-drop-out of treatment is related to a better outcome in physical and emotional functioning and pain severity [21]. Adherence is influenced by multiple factors such as the health care provider-patient relationship, the patient's self-efficacy to be able to make changes [22], and patient's satisfaction with improvement [23]. 
In order to improve adherence and motivation to prevent drop-out, and to strengthen selfefficacy, Motivational Interviewing (MI) has been proposed [24]. Miller and Rollnick, the founders of $\mathrm{MI}$, defined it as 'directive, client-centred counselling style for eliciting behaviour change by helping clients to explore and resolve ambivalence' [25]. The overall goal is to increase the client's intrinsic motivation to change and enhance behavioural change [26]. MI was originally developed for problem drinkers [27] and in the beginning applied in the addiction field only (e.g. [28, 29]). Meta-analyses showed that using $\mathrm{MI}$ as a pretreatment yielded the best outcomes compared with other active treatments [30-32]. Therein, MI was designed to prepare clients for further treatment such as CBT or an inpatient program. Furthermore, the effective application ranged from a variety of disorders such as addiction (except smoking cessation), increasing healthy behaviours, and the management of chronic illnesses. MI as treatment approach is fairly new in the field of chronic pain. MI has been applied with promising results in two small RCTs within primary care for patients with chronic pain conditions. Habib et al. (2005) found significant increases in attending self-management workshops after a psychologist-led 2-session MI-based feedback interview compared with an attention placebo interview [33]. Another recent study found a Ml-adapted intervention added to outpatient physiotherapy for patients with back pain effective in enhancing motivation and exercise adherence compared to physiotherapy alone [34]. With the results of these studies in mind, it seems promising to explore the effects of $\mathrm{MI}$ on non-adherence and drop-out in chronic pain rehabilitation care, and participation afterwards, where patients are characterized by high levels of disability and complex problems.

\section{Aims}

The primary objective of this current project is to study the effectiveness of MI by means of the MIP intervention. The intervention aims at decreasing drop-out rate and increasing adherence to treatment program to reach a high level of societal participation ultimately in patients with non-specific musculoskeletal chronic pain, who have been selected for pain rehabilitation treatment.

\section{The main research questions are:}

1. What is the effectiveness of MI-based pre-treatment (MIP) compared to usual care (UC) on the level of participation, treatment drop-out rate and adherence in patients with chronic non-specific musculoskeletal pain following pain rehabilitation?

2. What is the cost-effectiveness and cost-utility of MIP, compared to UC from a societal perspective?

3. What is the feasibility of the MIP intervention in terms of MI-fidelity (process evaluation)?

4. What are experiences of nurses and patients in terms of satisfaction with and barriers of the MIP intervention (process evaluation)?

\section{Hypotheses}

1a. It is expected that patients' level of social participation will be higher in the MIP intervention condition compared to the UC after pain rehabilitation treatment and at 6 months follow-up after finishing the rehabilitation treatment. 
1b. It is hypothesized that drop-out rates will be lower and adherence to the treatment and the level performing daily activities will be higher in the MIP condition compared to the UC.

2. It is hypothesized, that MIP will be more efficient compared to UC, both in effects as well as utilities.

3. It is hypothesized that the MIP intervention is feasible for the participating nurses and the MI-fidelity of the MIP intervention is sufficient.

\section{Methods/ Design}

\section{Study design}

The PREPARE study is a parallel single-blind randomized controlled trial (RCT).

\section{Recruitment}

Participants will be recruited from the outpatient department of Rehabilitation Medicine in an academic and a regional hospital in the Southern part of the Netherlands, starting in January 2012 and lasting till June 2013.

Patients visiting one of the participating outpatient rehabilitation departments for an intake interview will be evaluated whether they are eligible for rehabilitation treatment by the consultant in rehabilitation medicine.

\section{Participants}

Participants are patients who meet the inclusion criteria as stated below.

In current care, patients are selected for rehabilitation treatment by the consultant in rehabilitation medicine based on expert opinion. For this, both medical (origin and severity of the pain problem and interfering co-morbidity) and motivational factors are evaluated. In patients eligible for outpatient pain rehabilitation treatment, additional in- and exclusion criteria for participation in the PREPARE study are checked and in case of eligibility, patients will be invited to participate.

Inclusion criteria are: Non-specific chronic (duration $>3$ months) musculoskeletal pain; age between 18 and 65 years; the chronic pain syndrome is not attributable to a recognizable, known specific pathology (e.g. infection, tumour, osteoporosis, fracture, structural deformity, inflammatory disorder (e.g. ankylosing spondylitis); medium to high level of motivation for pain rehabilitation from the consultant's perspective; adequate literacy to complete assessment measures.

Exclusion criteria: Pregnancy; surgery planned in the foreseeable future; patient involved in litigation procedures; suspicion of a psychiatric disease that will interfere with rehabilitation treatment (according the expert opinion of the consultant in rehabilitation medicine). 


\section{Sample size}

The sample size calculation is based upon an estimated difference in change in level of participation between baseline and six months follow up (after completion of the rehabilitation treatment) as assessed by the Utrecht Scale for Evaluation of Rehabilitation-Participation (USER-P) [35, 36]. Reproducibility [37], responsiveness [38], and validity [37] of the USER-P are satisfactory. As participation is the important outcome in rehabilitation, we have chosen this as primary outcome. The scale consists of the three aspects of participation represented by the subscales Frequency, Restriction, and Satisfaction. The sum score of each subscale is converted to scores on a scale ranging from $0-100$. There is no USER-P total score.

Our sample-size calculation is based upon the subscale Satisfaction. This subscale is the most important part of participation as it marks the subjective experience of participation [9]; therefore we have chosen this one.

As at this moment no information is available about the USER-P in a pain population regarding normative values, clinically relevant change, and clinically relevant difference, our study is exploratory of character. In a study of van der Zee (2011) the USER-P is taken in different patient groups at three different points in time [38]. As these groups were part of a cohort study, nothing is known about differences after a specific treatment. We know from other studies that multidisciplinary pain rehabilitation treatment is moderate effective with effect sizes ranging from 0.30 for behavioural outcomes up to 0.40 for functional outcomes [14]. We expect an additional effect for the MIP-intervention since systematic reviews of Motivational interviewing-based interventions show an effect size ranging from 0.27 up to 0.40 when compared with a weak comparison group [30-32, 39]. It has to be said that in those reviews no study in the field of chronic pain or rehabilitation treatment was taken into account. Therefore, our power calculation is based on the following two assumptions:

1. Using an expected mean change of 5 points within UC is expected whereas a difference of additional 5 points (thus at least 10 points in total) within MIP in the USER-P sub scale Satisfaction is considered as clinically relevant. Those numbers are estimates as the clinically relevant difference is not known yet.

2. Assuming a standard deviation of 10 points in both groups, the standardized effect size, Cohen's $d$, is (10-5)/ 10=0.5 indicating a medium additional effect size of the MIP [40].

Assuming power $(1-\alpha)$ of $80 \%, \alpha=0.05$, two-sided testing, and holding the above mentioned assumptions, would necessitate $n=128$ participants ( $n=64$ per arm). With an expected total dropout of 20\% (10\% drop-out from the study, another 10\% drop-out during the usual rehabilitation treatment as our unsystematic registration has shown), $n=160$ ( $n=80$ per arm) was chosen as the optimal total sample size. Additionally, as the recruitment of 160 participants in the region of South-Limburg/ the Netherlands is feasible in a time range of 18 months, we have chosen for this amount of participants. 


\section{Informed consent, randomisation, and blinding}

Standard informed consent procedures will be used. After having received informed consent, the participants are randomly assigned to either the experimental intervention condition (MIP) or the control condition (UC). Block randomisation with block size of four are used per study site (hospital) to obtain equal numbers in both arms. An independent research assistant will execute the randomisation by means of a computerized random number generator resulting in a computer-generated list of random numbers to allocate the participant either to the experimental or the control condition. The consultant in rehabilitation medicine, the rehabilitation team and the participant will all be blind for the result of the allocation to the UC or the MIP but not for participation in the study at all. The research assistant responsible for the logistics of the present study and the participating nurses administering the intervention and control condition are not part of the treatment team and are not blinded for allocation.

\section{Treatment}

To understand the features of both PREPARE conditions, first current care in pain rehabilitation in the Netherlands will be explained. Thereafter, both PREPARE conditions are described. At last, the role of the nurses is illustrated.

\section{Current care in pain rehabilitation}

In the current pain rehabilitation treatment in the Netherlands [41] roughly four phases can be identified. The specific content of the phases can differ per center.

\section{The four phases are:}

1. Screening: In this phase, a patient's eligibility and readiness for pain rehabilitation is assessed. Also information is provided by either a consultant in rehabilitation medicine or a nurse practitioner. Most centres for this purpose use existing educational material, such as 'De pijn de baas' ('Mastering pain') [42] or 'Explain Pain' [43] (WPN, 2012, internal document) as information tools. In most places, information is given before the definite start of the treatment.

2. Observation: A patient's pre-treatment situation and motivation is assessed, and the most suitable type of treatment is selected. In some centres, this phase takes two or three sessions in two to four weeks. Often this is accompanied by the use of self-report questionnaires.

3. Rehabilitation treatment: The multidisciplinary pain rehabilitation treatment lasts for around three months with an intensity of two or three days a week on average. Depending on the patients' limitations and underlying problems, different treatment approaches such as graded activity and/ or graded exposure are used in combination with other psychosocial and physical interventions. 
4. Aftercare and post rehabilitation treatment assessment: Booster sessions, tailored to patient's needs, are given for relapse prevention and long-term facilitation of applying learned skills to maintain and further improve participation. The moment of the assessment is around 6, 12, 24 to 52 weeks post-treatment. These follow-up appointments are mostly accompanied by completion of self-report questionnaires for treatment evaluation. It has to be said that this is not routine care in all Dutch rehabilitation centres.

\section{PREPARE conditions}

The PREPARE intervention focuses on phase one. This is also the period during which patients are waiting before they can start their actual treatment (observation and rehabilitation treatment). During the study, patients will receive either the new Ml-based intervention or care as usual. In order to standardize treatment options, both arms (MIP as well as UC) will include two sessions with identical contact time. In order to prevent contamination of treatment, each treatment condition will be delivered by different nurses. Figure 1 illustrates the situation during the PREPARE study (see next page). A comparison of the features of both conditions is given in table 1.

\section{Usual care (UC)}

In the usual care (UC) arm, participants will receive pain education according to the information 'De pijn de baas' [40] (Mastering pain). The ultimate goal is to provide the participant with information about e.g. differences between acute and chronic pain, its nature, and strategies to handle cognitions about pain.

\section{MIP intervention condition}

The MI intervention condition is based on the four general principles of MI, which are incorporated into all sessions in the MIP-condition [44]:

1. Expressing empathy by the use of reflective listening,

2. Developing discrepancy between client goals and current problem behaviour,

3. Rolling with resistance by avoiding argumentation by assuming that the client is responsible for the decision to change,

4. Supporting self-efficacy and optimism for change [44].

The nurse is directive within this process, but the participant's autonomy is strengthened and his or her right to decide on everything related to the person is respected.

During the 1st appointment, a trustful relationship between participant and nurse is built, the actual (life) situation, burden and impairments of the chronic pain in daily life, motivation, self-efficacy, and readiness to change behaviour is assessed and enhanced. Next, the session is summarized and closed. The 2nd appointment starts with a brief motivational feedback session. The process of the 1st appointment will be discussed with the participant by giving feedback adapted to the state of readiness-to-change. Therein, motivation and self-efficacy for behaviour change is enhanced. In addition, topics related to chronic pain and treatment, such as educa- 
tion about the influence of exercise and a background in the bio psychosocial approach will be discussed. Next, the session is summarized and closed.

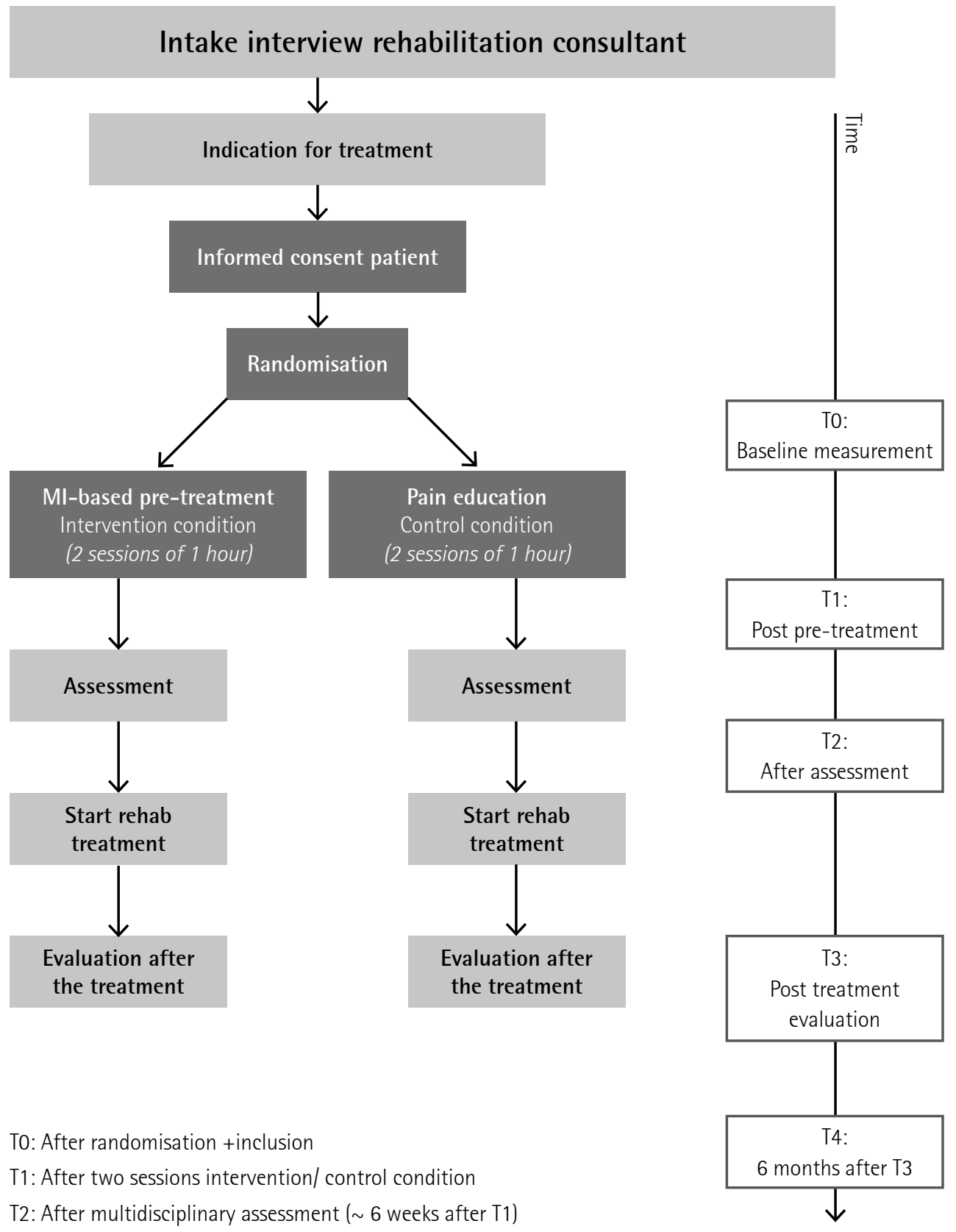

Figure 1: Flow-chart of the design of the PREPARE study 
Table 1: Features of the two interventions

\begin{tabular}{|c|c|c|}
\hline $\begin{array}{l}\text { Motivational interviewing } \\
\text { based pre-treatment (MIP) }\end{array}$ & $\begin{array}{l}\text { Pain education } \\
\text { (Usual Care, UC) }\end{array}$ & \\
\hline $\begin{array}{l}\text { Goal: explore participant's } \\
\text { life situation, impairments } \\
\text { and ambivalences in order to } \\
\text { enhance intrinsic motivation. }\end{array}$ & $\begin{array}{l}\text { Goal: provide the participant } \\
\text { with information. }\end{array}$ & Goal \\
\hline General principles of MI. & $\begin{array}{l}\text { General principles of he- } \\
\text { alth education and patient } \\
\text { education. }\end{array}$ & Basic principle \\
\hline $\begin{array}{l}\text { Content based on patient-dri- } \\
\text { ven topics derived from the } \\
\text { ICF-model. }\end{array}$ & $\begin{array}{l}\text { Content based on the book } \\
\text { 'De pijn de baas'[42] (Maste- } \\
\text { ring pain). }\end{array}$ & Foundation \\
\hline $\begin{array}{l}\text { Content sessions tailored to } \\
\text { the patients' readiness to } \\
\text { change. }\end{array}$ & $\begin{array}{l}\text { Content is fixed by means of } \\
\text { the treatment protocol. }\end{array}$ & Protocol rigidity \\
\hline $\begin{array}{l}\text { Exploration actual (life) situa- } \\
\text { tion, burden and impairments } \\
\text { of the chronic pain in daily } \\
\text { life. } \\
\text { Assessing +enhancing } \\
\text { motivation, self-efficacy, } \\
\text { and readiness to change for } \\
\text { behaviour. }\end{array}$ & $\begin{array}{l}\text { Provision of general health } \\
\text { education about topics rela- } \\
\text { ted to chronic pain. } \\
\text { Provision of information re- } \\
\text { garding core elements of pain } \\
\text { rehabilitation. }\end{array}$ & Content $1^{\text {st }}$ session \\
\hline $\begin{array}{l}\text { Giving feedback adapted to } \\
\text { the state of readiness-to-ch- } \\
\text { ange. } \\
\text { Continuation exploration. } \\
\text { Continuation assessing +en- } \\
\text { hancing motivation, self-effi- } \\
\text { cacy, and readiness to change } \\
\text { for behaviour. }\end{array}$ & $\begin{array}{l}\text { Continuation of general } \\
\text { health education about topics } \\
\text { related to chronic pain. }\end{array}$ & Content $2^{\text {nd }}$ session \\
\hline $\begin{array}{l}\text { Is given related to the stage of } \\
\text { change of the participant ac- } \\
\text { cording to the } \mathrm{Ml} \text {-principles. }\end{array}$ & Is not given. & Feedback \\
\hline
\end{tabular}




\section{Nurses}

Both UC and MIP are administered by registered nurses working in the field of rehabilitation. In order to prevent contamination of treatment by components of the other intervention, nurses will be trained and only guide participants in one specific condition. The two nurses selected to deliver the MI condition, are both experienced MI coaches (four years of experience). To assure optimal quality of the $\mathrm{Ml}$ condition, both nurses receive an expert training by a certified Ml-trainer: Their Ml-knowledge and experience in the context of chronic pain rehabilitation are updated based on an evidence based training tailored to their specific needs [45-49]. During the study the Ml-sessions will also be audio taped and the Ml-quality will be assessed using a coding instrument (Motivational Interviewing Treatment Integrity, MITI) (see also section methods, section process evaluation). Subsequent training will be based on additionally regular supervision, done by training-on-the job and direct feedback on MIP sessions.

\section{Quality of the UC-condition}

The two nurses selected to deliver the usual care condition are experienced in the field of rehabilitation (20 years, and five years, respectively).

Before the start of the study, they will receive a three-hour refresher training in general communication skills and general principles of health education. In addition, the content of relevant chapters of the book 'Mastering pain' is discussed. During the study, the nurses of the UC condition will meet one to two times a year to discuss problems encountered during the sessions.

\section{Data collection}

For the (cost)effectiveness evaluation, measurements will be carried out before the first MIP/ UC pre-treatment sessions (T0), after the MIP/ UC ( $T 1$ = post pre-treatment), after the regular multidisciplinary pre-rehabilitation assessment (before starting rehabilitation), at the start of the pain rehabilitation treatment (T2), after treatment completion (T3), and six months follow up (T4). Study assessments at T0, T2 and T3 will be integrated in current care clinical assessment battery pre-rehabilitation and post rehabilitation (see also figure 1 (flow-chart) and table 2). Cost measurements will be carried out at three moments: T0, T2 and T4. Information on drop-out from and adherence to the (pre-) rehabilitation treatment, start and end date of the treatment and no show are derived from the administrative patient registry.

As patients have to incorporate learned behaviour into daily routine functioning, the improvement of participation, the ultimate goal of pain rehabilitation, is not optimal directly after treatment. We expect relevant change in the level of participation to occur at 6 months. Therefore this time point is chosen as our last measurement moment. Due to the fact that the Prepare trial is embedded in clinical practice, the measurement moments are not precisely planned at the same moment. Especially the waiting list period between T1 and T2 may vary and furthermore the total duration of the pain rehabilitation treatment may vary between 10-16 weeks. 
Table 2: Overview outcome measures, abbreviation instrument, and assessment moments PREPARE study
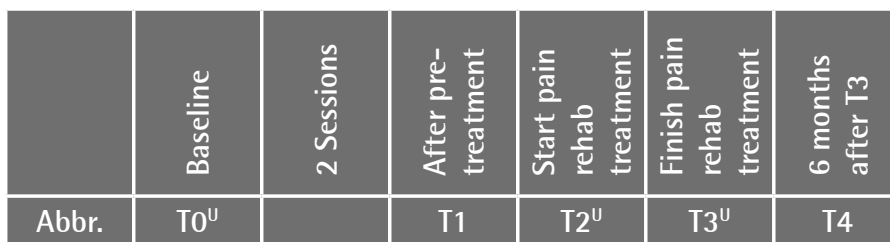

\begin{tabular}{l}
\hline Covariates \\
\hline Demographics patient \\
\hline Primary outcome effect \\
evaluation
\end{tabular}

Participation
Secondary outcomes effect
evaluation

\begin{tabular}{l} 
Physical functioning/ disability \\
\hline Pain intensity \\
\hline Main complaints
\end{tabular}

Main complaints

Drop-out/ adherence

Self-efficacy/ perceived com-
petence

Pain-specific self-efficacy scale

USER-P $\quad X$

\section{Cost-effectiveness evaluation}

Cost questionnaire

Productivity losses

Quality of life/ health status

\begin{tabular}{|l|l|}
\hline PDI & $\mathbf{X}$ \\
\hline VAS & $\mathbf{X}$ \\
\hline PSC & $\mathbf{X}$ \\
\hline
\end{tabular}

Potential active ingredients

MI

Stages of change
MI-integrity/fidelity
Potential moderators

Credibility and expectancy of the treatment

\begin{tabular}{|l|l|}
\hline Motivation & TMO \\
\hline Depression & BD \\
\hline Acceptance & A \\
\hline Flexible goal-adjustment & FGA \\
\hline Process evaluation & \\
\hline
\end{tabular}

\begin{tabular}{|l|l|}
\hline Tic-P & $\mathbf{X}$ \\
\hline SF-HLO & $\mathbf{X}$ \\
\hline SF-36 & $\mathbf{X}$ \\
\hline
\end{tabular}

$$
\mathrm{X}
$$

$\mathrm{X}$

$\mathrm{X}$

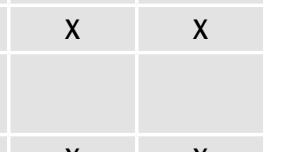

GSE $\quad \mathrm{X}$

\begin{tabular}{|l|l|l|}
\hline CPSS_V2 & $X$ & $x$ \\
\hline
\end{tabular}

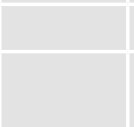

MPR

\begin{tabular}{|l|l|}
\hline MPRCQ 2 & $\mathrm{X}$ \\
\hline
\end{tabular}

\begin{tabular}{|l|l|l|}
\hline $\mathrm{X}$ & $\mathrm{X}$ & $\mathrm{X}$ \\
\hline $\mathrm{X}$ & $\mathrm{X}$ & $\mathrm{X}$ \\
\hline $\mathrm{X}$ & $\mathrm{X}$ & $\mathrm{X}$ \\
\hline
\end{tabular}

\begin{tabular}{|l|l|l|l|}
\hline & $\mathrm{X}$ & $\mathrm{X}$ & $\mathrm{X}$ \\
\hline$\#$ & & $\#$ & \\
\hline $\mathrm{X}$ & & $\mathrm{X}$ & $\mathrm{X}$ \\
\hline & & & \\
\hline
\end{tabular}

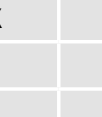

\begin{tabular}{|l|l|}
\hline$x$ & $x$ \\
\hline & \\
\hline
\end{tabular}

\begin{tabular}{l} 
Satisfaction with (pre-)treat- \\
ment \\
Client-centeredness \\
\hline Feasibility
\end{tabular}

\begin{tabular}{|l|l|l|}
\hline CEO & $x$ \\
\hline
\end{tabular}

\begin{tabular}{|l|l|l|}
\hline $\mathrm{X}$ & & $\mathrm{X}$ \\
\hline $\mathrm{X}$ & $\mathrm{X}$ & $\mathrm{X}$ \\
\hline & & \\
\hline & & \\
\hline
\end{tabular}

\begin{tabular}{|l|l|}
\hline TMQ & $\mathbf{X}$ \\
\hline BDI & $\mathbf{X}$ \\
\hline AAQ-II & $\mathbf{X}$ \\
\hline FGA & $\mathbf{X}$ \\
\hline
\end{tabular}

\section{$\mathrm{X}$}

\begin{tabular}{|l|l|l|}
\hline & $\mathrm{X}$ & $\mathrm{X}$ \\
\hline $\mathrm{X}$ & & $\mathrm{X}$ \\
\hline $\mathrm{X}$ & & $\mathrm{X}$ \\
\hline
\end{tabular}

$\mathrm{X}+$

$\mathrm{X}$

$+$ 


\section{Demographic and medical variables}

Baseline assessment includes: Gender, nationality, marital status, educational level, and co-morbidity as well as pain location and pain duration.

In addition, baseline assessment includes the following risk factors for attrition: sick leave [50], level of being active in sports, smoking status [18], level of physical functioning [50], pain intensity [50,51], perceived disability [51], low treatment satisfaction [52], and expectations regarding the content of the treatment [53].

\section{Outcome measures}

Outcome measures (parameters) and assessment moments are presented in table 2.

\section{Primary outcome}

The primary outcome of the effect-evaluation will be the mean difference in change in level of participation of the participants at T4 compared to T0 (baseline).

Participation will be measured by the Utrecht Scale for Evaluation of Rehabilitation-Participation (USER-P) [54]. It consists of 32 items and covers three aspects of participation namely Frequency, Restriction, and Satisfaction. The subscale Frequency consists of twelve items and assesses vocational activities, and frequency of leisure activities and social activities. Each item is scored from 0 ('not at all') up to 5 ('36 hours or more'/ '19 times or more'). The subscale Restriction comprises 10 items assessing experiences in vocational and leisure activities. Items are rated on a 4-point Likert scale ranging from 0 'not possible at all' to 3 ('independent without disability'). The subscale Satisfaction consists of 10 items and rates satisfaction regarding participation in daily life issues such as work and social relations. Items are rated on a five-point Likert scale ranging from 0 ('not satisfied at all') to 4 ('very satisfied'). Higher scores reflect more social participation (each higher frequency, less restrictions, higher satisfaction). The psychometric qualities validity and reproducibility are satisfactory $[36,55]$.

\section{Secondary outcomes}

Level of performing daily activities resulting from chronic pain will be measured by the Pain Disability Index (PDI) [56], which consists of seven items using an 11-point Likert scale ranging from 0 (no disability) to 10 (total disability). A total score is derived by summing up the item responses, ranging from 0 up to 70, with a higher score indicating more disability [56]. Good psychometric properties of the PDI have been shown and normative data are available [57].

Pain intensity is assessed by a 100-mm Visual Analogue Scale (VAS). The VAS is a common and valid tool for measuring pain intensity [58].

In order to assess the participant's self-reported main complaints, the Patient Specific Complaints questionnaire (PSC) will be used $[59,60]$. The participant selects 3 to 5 most limited functional activities and rates the difficulty to perform them during the previous week on a 100-mm Visual Analogue Scale (VAS) [60]. It is tested as valid, reliable and responsive in patients with chronic musculoskeletal pain $[61,62]$.

Drop-out will be registered in the patient registry in the institution. Each patient will be classified 
as either a participant who has completed the pain rehabilitation program as proposed or who has dropped out prematurely. In addition, the attendance of each treatment session will be assessed. The level of adherence to the pre-treatment intervention (MIP and UC) is computed by dividing the number of the PREPARE sessions that an individual participant actually has attended by the number of two pre-treatment sessions as planned.

Adherence to the rehabilitation treatment is computed by dividing the number of scheduled treatment sessions (which amount is tailored to the participants' needs) by the actual attended number of visits. This method has been reported extensively in the literature as a measure of adherence to rehabilitation [63].

General self-efficacy is assessed by the Dutch General Self-efficacy questionnaire [64]. Ten items ranging from 'totally untrue' up to 'totally true' assess coping in general life demands. A confirmatory factors analysis has taken place in elderly [65] showing a good fit.

A disease-specific self-efficacy measure is used, namely the Chronic Pain Self efficacy Scale (CPSS) consisting of 22 items the three subscales, pain management, coping and physical function. It offers good overall internal consistency and acceptable test-retest reliability [66].

\section{Cost-effectiveness and cost-utility}

To evaluate the economic effects of MIP and UC, relevant cost categories of resource use and volumes of these categories will be measured. Finally, volumes are multiplied by the corresponding costs.

The direct (non-)health care costs will be assessed with the Trimbos/iMTA questionnaire for Costs associated with Psychiatric Illness (Tic-P) [67]. This self-reporting questionnaire consists of 15 items and assesses health care use in a recall period of 3 months.

Productivity losses are assessed by the Short Form Health and Labor Questionnaire (SF-HLO) [68]. The SF-HLO measures the extent of production losses of paid and unpaid work in four modules: absence from work, reduced productivity at paid work, unpaid labor production and impediments to paid and unpaid labor. It consists of 11 items.

For the cost valuation, standardized cost prices will be used from the Dutch manual for cost analysis in health care research [69]. Where no standard cost prices are available, real costs or tariffs will be used to estimate costs. Productivity losses will be calculated based on the friction costs method. Cost prices will be presented in Euros and the baseline year is 2012, or otherwise discounted. The discounting rate of costs is $4 \%$, and $1.5 \%$ for effects [70]. To analyze differences in costs, costs per patient-year will be calculated.

For the cost-effectiveness analysis (CEA), costs will be weighted against the primary outcome measure participation. The measure is described above.

For the cost-utility analysis (CUA), costs per year will be weighed against utilities based on the SF-36. The SF-36 is a reliable and valid instrument to measure health related quality of life [71]. Utilities are values or preferences that respondents assign to a particular health state and are overall expressed on a scale ranging from 0 to 1 [72]. The utilities used in this study will be derived with an algorithm of the SF-6D, which estimates utilities based on the health related quality of life scores of the SF-36 [73]. Furthermore, the utilities will be estimated by an algorithm 
of Brazier, Roberts and Deverill (2002). The derived utilities at three measurement points (T0, T2, T4) will be used to compute the Quality Adjusted Life Years (OALY) score by means of the area under the curve method [74]. This means that the weights for each health state (utilities) will be multiplied by the time in that particular health state and then summed to calculate the total number of $Q A L Y S$.

\section{MI-specific possible active ingredients}

Resulting from the existing literature on $\mathrm{MI}$, potential specific active ingredients were identified and will be assessed in this study $[75,76]$.

The stage of change is assessed by the Multidimensional Pain Readiness to Change Questionnaire 2 (short version). It measures the chronic pain participants' readiness to change. The questionnaire is based upon the trans theoretical model of behaviour change of Prochaska and DiClemente. The MPRCO2-26 consists of 26 items and is scored on a 7-point Likert scale [77]. Psychometric properties are evaluated and appeared to be satisfactory [78]. To be able to take it into account as possible moderator, it is measured before and after the pre-treatment.

MI-integrity is hypothesized to be another working mechanism for MI. It is further explained below under the heading 'Ml integrity and fidelity'.

\section{Possible moderators}

We will also measure specific individual factors at baseline that could influence the treatment effect. From the literature, a number of psychosocial attributes moderating the effects of the pain rehabilitation treatment are identified [79].

Research has shown the influential role of expectancy on treatment outcome in pain rehabilitation $[80,81]$. Credibility and expectancy is measured by an adapted and translated version of the Credibility and Expectancy Questionnaire (CEQ)[82]. In two parts, participants are asked to rate in total five items related to the credibility and expectancy regarding pain rehabilitation treatment. Credibility and expectancies regarding the treatment have to be rated in two items. In another item the expected success in terms of an improvement in participation, decrease in disability, and decrease in pain intensity are rated. The CEO uses a rating scale, ranging from 1 (not at all) to 9 (very much). Good psychometric properties have been demonstrated and the same factors structure was confirmed [82].

Motivation is assessed by the Treatment Motivation Questionnaire (TM0). The TM0 assesses intrinsic and extrinsic information about entering and remaining in treatment [83]. It consists of 26 items to be rated on a five-point Likert scale ranging from 1 (not at all true) up to 5 (very much true). The factors internal and external motivation, interpersonal help seeking and confidence in treatment are represented. Items are slightly adapted to the rehabilitation context. The TM0 correlates well with professionals' rating of the above mentioned factors what suggests good construct validity [83].

Depressive symptoms are hypothesized to moderate the effects of the rehabilitation outcomes. Therefore, the level of depression is assessed by the Becks Depression Inventory (BDI)[84]. The BDI is a well-known instrument, and suitable for its use in pain research. It is a reliable, valid and 
widely used instrument $[85,86]$.

Acceptance has shown to explain a significant amount of variance in the prediction of patient functioning and suffering from chronic pain [87]. Acceptance is assessed by the 10-item Acceptance and Action Questionnaire-II (AAQ-II)[88] and scored on 7-point Likert scale items ranging from 'never be true' up to 'always true.' The Dutch version has shown a high internal consistency and a good validity [89].

Flexible goal-adjustment has shown to be a moderator in the relation between self-discrepancies and negative emotions [90]; flexibility has shown to be of moderating influence on pain willingness and activity engagement [91]. Flexible goal-adjustment (FGA), a component of psychological flexibility is measured with the Tenacious Goal Pursuit and Flexible Goal Adjustment Scale Brandstädter and Renner Questionnaire [92], which assesses the tendency to adjust personal goals and standards to situational limitations. It consists of 15 items with belonging five possible answers ranging from 'totally agree' up to 'not agree at all'. The scale's internal consistency is satisfactory $(\alpha=0.80)$ [92].

\section{Process evaluation: MI integrity and MI fidelity}

To check whether MI was implemented and delivered as intended, an evaluation of treatment fidelity and treatment integrity is important.

Also given the explicit emphasis in MI as a spirit rather than a technique [93], treatment fidelity and treatment integrity will be measured. Therefore, the MI Treatment Integrity Code (MITI version 3.1) [94] will be used. It has shown to be a cost-effective and reliable tool $[95,96]$, which is also validated to check MI-fidelity $[94,97]$. Feedback during the training and during intervision sessions will be delivered based also on the MITI. Research has also shown that proficiency rating by skilled coders predicted treatment outcome [98-100]. To be able to do so, all pre-treatment sessions are audio taped, and a random sample of $20 \%$ of all sessions will be scored by one of the authors (VCM). Twenty per cent out of those will also be scored by another skilled coder (N.N) using the abovementioned MITI instrument.

\section{Process evaluation in participants and nurses}

A process evaluation is needed to evaluate the actual delivery of our pre-treatment intervention, the process evaluation focuses on the level of nurses and patients [101].

1. Patients: Patients will be asked to score their satisfaction with pre-treatment in a set of questions for process evaluation purposes at the end of the pre-treatment intervention (included in the questionnaire of T1). In case of treatment drop-out, a patient is asked to answer the questions regarding satisfaction with the (pre-)treatment directly after the decision to stop, including reasons for drop-out from treatment.

Furthermore, client-centeredness will be assessed by the Client Centred Care Questionnaire (CCCO). The questionnaire was originally developed for the use in clients receiving homecare [102]. By making some minor revisions, it can also be used in the rehabilitation setting [103]. It has shown good psychometric qualities [102].

2. Nurses: After both pre-treatment sessions, nurses are asked to fill in a short questionnaire. 
Questions are asked about the steps taken during the intervention, the content of the discussion during the sessions, and the client's active participation. The questionnaire's structure is based on the work of Steckler and Linnan [101] and adapted to the specific situation of our study.

\section{Statistical analysis}

The statistical analysis for the effect evaluation, the cost-effectiveness evaluation and cost-utility analysis, and the process evaluation will be described separately. Data will be analysed using SPSS version 17.

\section{Effect evaluation}

Baseline data will be analysed to describe the characteristics of all participants and to check for significant imbalances between the two groups. Possible baseline differences between the intervention group and the control group will be tested by an independent samples t-test (normal distribution) or Mann Whitney u-tests (non-normal distribution) in the case of continuous variables. In the case of dichotomous variables, a Chi-square test will be used. In case of imbalances in prognostic factors between both randomized groups, an adjustment for those factors will take place. The number of drop-outs and follow-up losses in both groups will be reported based on descriptive data. Differences in primary and secondary outcome measures between MIP and UC will be analysed using repeated measurements techniques and multilevel analysis. This approach is chosen because repeated measures within individual subjects are taken. A p-value $<0.05$ is considered statistically significant. Data will be analyzed according to the intention-to-treat principle. All outcomes will be assessed at each follow-up moment by taking into account a specific a priori hypothesis. Additionally, per protocol analyses will be performed to assess whether protocol deviations have caused bias.

\section{Moderation analysis}

As secondary analyses, the working mechanisms of $\mathrm{MI}$ are investigated. For testing moderation, an interaction term of the potential moderator is constructed and analyzed by linear regression.

\section{Economic evaluation}

In the economic evaluation, cost and effects of usual care and MIP will be calculated and compared. Therefore, the cost per patient year (=participant year) will be calculated. This means that all observed costs will be extrapolated to a one-year period.

The analysis will include those persons for whom at least two of the three follow-up measurements (cost diaries) are available.

For the cost-effectiveness analysis (CEA) the cost-effectiveness ratio will be stated in terms of costs per improvement on participation (as assessed with the USER-P).

For the cost-utility analysis (CUA) the cost-utility ration will be stated based on the cost per (Quality Adjusted Life Year) QALY gained. Bootstrap (5000 times) re-sampling techniques will be used to test for differences and uncertainty in costs and effects between the MIP and the UC intervention. 
The bootstrapped cost-effectiveness and cost-utility ratios will be subsequently plotted in a cost-effectiveness and cost-utility plane. The results of this study will also be depicted in cost-effectiveness acceptability curves (CEACs) [104].

\section{Process evaluation}

Results of the questionnaires for the process evaluation of participants and nurses will be analyzed descriptively. A summary score of Ml-fidelity will be computed by ratings of the Ml-consistent behavior evaluated by the MITI. Summary score thresholds are known for beginning proficiency level and competency level [94]. A binary variable MI-fidelity will be computed and used in analyses for the effectiveness of Ml as well as working mechanisms of MI. MI-fidelity is furthermore used in mediation analyses as hypothesized mediator in the path between the Ml-intervention and its effect.

\section{Discussion}

During the design of this study many choices and selections were made, and three of the most crucial ones will be discussed.

First, we specifically chose for participation as our primary outcome measure instead of for example disability. This is in accordance with the main outcome as suggested in the (International Classification of Functioning, Disability and Health) ICF. The ICF is nowadays accepted in most countries to describe and measure health and disability [105], and the relevant aim of rehabilitation. Therefore, it is pre-eminently adequate to use participation as primary outcome measure in the field of rehabilitation [106]. The choice of the other current outcome measures is congruent with the IMMPACT recommendations [10, 107, 108].

Second, we chose for an attention control group instead of a no-treatment group. Reasons for our choice are: As we hypothesize that attention of health providers alone could be a factor associated with motivation for treatment, it seems important to standardize for this factor when studying the effect of the content of a new intervention aiming at changing motivation for treatment. Therefore, we preferred the design of a trial comparing a Ml-based and an attention control pain education above the design of a trial with a single intervention and one waiting list condition without control intervention. Since the content of pain education is part of usual care in the Netherlands, we designed an attention control intervention based on the content of usual care. For comparability reasons, the duration of the attention control condition is identical to the Ml-intervention.

The quality and the efficacy of MI delivery are troublesome within some research reports, and the documentation of MI fidelity is often lacking (e.g. $[34,109])$. Therefore, we lay a strong emphasis on the Ml-training of the care providers and an on-going Ml-fidelity check. 
MI-training is provided in order to make nurses adequately proficient to learn the principles and acquire the skills of $\mathrm{MI}[46,99,110,111]$. MI-integrity is checked alongside the trial for two aims: For feedback about MI-proficiency during intervision with the nurses [95] and for the interpretation of $\mathrm{Ml}$ effects since intervention fidelity is an important prerequisite for intervention effects. To conclude, this paper describes the design of a randomized controlled trial to study the effectiveness of a nurse-led Motivational Interviewing-based pre-treatment intervention aimed at improving and sustaining participation up until 6 months after the pain rehabilitation. The results of this study will provide evidence of the effectiveness of this pre-treatment intervention as well as insights in working mechanisms as well as cost-effectiveness and cost-utility and moderating mechanisms of such an intervention in a chronic pain population.

\section{Ethical approval}

The study protocol was approved by the Medical Ethical Committee of University Hospital Maastricht and Maastricht University. The study is registered in a public trial registry (Nederlands Trial Register NTR3065).

\section{Trial status}

Prepare is an on-going trial and it is expected that patient recruitment will last till approximately June 2013.

\section{List of abbreviations}

\section{Abbreviation}

\begin{tabular}{|l|l|}
\hline MI & Motivational Interviewing \\
\hline MIP & Motivational Interviewing-based pre-treatment \\
\hline Prepare & Pre-Pain rehabilitation \\
\hline UC & Usual Care \\
\hline
\end{tabular}

\section{Competing interests}

This PhD project is funded by Adelante zorggroep, Hoensbroek, the Netherlands.

\section{Authors' contributions}

VCM, MG, JV, AK, and RS participated in the concept, design of the study and research protocol. All authors read and corrected draft versions of the manuscript and approved the final manuscript.

\section{Acknowledgments}

We thank Wilma Noteborn, the MI-trainer, for her valuable comments on the MIP-protocol. 


\section{References}

[1] Gran JT: The epidemiology of chronic generalized musculoskeletal pain. Best Pract Res Clin Rheumatol 2003, 17:547-561.

[2] Badley EM, Webster GK, Rasooly I: The impact of musculoskeletal disorders in the population: are they just aches and pains? Findings from the 1990 Ontario Health Survey. J Rheumatol 1995, 22:733-739.

[3] Meerding WJ, Bonneux L, Polder JJ, Koopmanschap MA, van der Maas PJ: Demographic and epidemiological determinants of healthcare costs in Netherlands: cost of illness study. BMJ 1998, 317:111-115.

[4] Koes BW, van Tulder MW, Thomas S: Diagnosis and treatment of low back pain. BMJ 2006, 332:1430-1434.

[5] Köke A: Consensus Rapport Pijnrevalidatie Nederland [Consensus Report Pain rehabilitation Netherlands]. Maastricht: Pijn Kennis Centrum Maastricht [Pain Knowledge Centre Maastricht]; 2005.

[6] Bekkering GE, Hendriks HJM, Koes BW, Oostendorp RAB, Ostelo R, Thomassen JMC, van Tulder MW: Dutch Physiotherapy Guidelines for Low Back Pain. Physiotherapy 2003, 89:82-96.

[7] Gatchel RJ, Peng YB, Peters ML, Fuchs PN, Turk DC: The biopsychosocial approach to chronic pain: scientific advances and future directions. Psychol Bull 2007, 133:581-624.

[8] Turk DC, Wilson HD, Cahana A: Treatment of chronic non-cancer pain. Lancet 2011, 377:2226-2235.

[9] International Classification of Functioning, Disability, and Health (ICF). ICF full version. Geneva, Switzerland: World Health Organization; 2001.

[10] Dworkin RH, Turk DC, Farrar JT, Haythornthwaite JA, Jensen MP, Katz NP, Kerns RD, Stucki G, Allen RR, Bellamy N, et al: Core outcome measures for chronic pain clinical trials: IMMPACT recommendations. Pain 2005, 113:9-19. 
[11] Keith RA: Conceptual Basis of Outcome Measures. Am J Phys Med Rehabil 1995, 74:73-80.

[12] Dijkers MP, Whiteneck G, El Jaroudi R: Measures of social outcomes in disability research. Arch Phys Med Rehabil 2000, 81:S63-80.

[13] Morley S, Eccleston C, Williams A: Systematic review and meta-analysis of randomized controlled trials of cognitive behaviour therapy and behaviour therapy for chronic pain in adults, excluding headache. Pain 1999, 80:1-13.

[14] van Tulder MW, Ostelo R, Vlaeyen JW, Linton SJ, Morley SJ, Assendelft WJ: Behavioral treat ment for chronic low back pain: a systematic review within the framework of the Cochrane Back Review Group. Spine 2000, 25:2688-2699.

[15] Henschke N, Ostelo RW, van Tulder MW, Vlaeyen JW, Morley S, Assendelft WJ, Main CJ: Behavioural treatment for chronic low-back pain. Cochrane Database Syst Rev 2010:CD002014.

[16] Guzman J, Esmail R, Karjalainen K, Malmivaara A, Irvin E, Bombardier C: Multidisciplinary bio-psycho-social rehabilitation for chronic low back pain. Cochrane Database Syst Rev 2002:Cd000963.

[17] Sabate E: Adherence to long-term therapies. Evidence for action. Geneva: WHO; 2003.

[18] Bendix AE, Bendix T, Haestrup C, Busch E: A prospective, randomized 5-year follow-up study of functional restoration in chronic low back pain patients. Eur Spine J 1998, 7:111-119.

[19] Peters J, Large RG, Elkind G: Follow-up results from a randomised controlled trial evaluating in- and outpatient pain management programmes. Pain 1992, 50:41-50.

[20] Rainville J, Ahern DK, Phalen L: Altering beliefs about pain and impairment in a functionally oriented treatment program for chronic low back pain. Clin J Pain 1993, 9:196-201.

[21] Curran C, Williams AC, Potts HW: Cognitive-behavioral therapy for persistent pain: does adherence after treatment affect outcome? Eur J Pain 2009, 13:178-188.

[22] Bandura A: Self-efficacy: The exercise of control. New York: WH Freeman; 1997.

[23] Hirsh AT, Atchison JW, Berger JJ, Waxenberg LB, Lafayette-Lucey A, Bulcourf BB, Robinson ME: Patient satisfaction with treatment for chronic pain: predictors and relationship to compliance. Clin J Pain 2005, 21:302-310. 
[24] Jones KD, Burckhardt CS, Bennett JA: Motivational interviewing may encourage exercise in persons with fibromyalgia by enhancing self efficacy. Arthritis Care Res 2004, 51:864-867.

[25] Miller WR, Rollnick S: Motivational interviewing, preparing people to change addictive behavior. New York: The Guildford Press; 1991.

[26] Mesters I: Motivational interviewing: hype or hope? Chronic IIIn 2009, 5:3-6.

[27] Miller WR: Motivational interviewing with problem drinkers. Behav Psychother 1983, 11:147-172.

[28] Heather N, Rollnick S, Bell A, Richmond R: Effects of brief counselling among male heavy drinkers identified on general hospital wards. Drug Alcohol Rev 1996, 15:29-38.

[29] Skutle A, Berg G: Training in Controlled Drinking for Early-stage Problem Drinkers. Br J Addict 1987, 82:493-501.

[30] Burke BL, Arkowitz H, Menchola M: The efficacy of motivational interviewing: a meta-analysis of controlled clinical trials. J Consult Clin Psychol 2003, 71:843-861.

[31] Hettema J, Steele J, Miller WR: Motivational interviewing. Annu Rev Clin Psychol 2005, 1:91-111.

[32] Lundahl B, Burke BL: The effectiveness and applicability of motivational interviewing: A practice-friendly review of four meta-analyses. J Clin Psychol 2009, 65:1232-1245.

[33] Habib S, Morrissey S, Helmes E: Preparing for pain management: a pilot study to enhance engagement. J Pain 2005, 6:48-54.

[34] Vong SK, Cheing GL, Chan F, So EM, Chan CC: Motivational enhancement therapy in addition to physical therapy improves motivational factors and treatment outcomes in people with low back pain: a randomized controlled trial. Arch Phys Med Rehabil 2011, 92:176-183.

[35] van der Zee CH, Post MWM, Kap A, Schouten E, van Royen R, Rambaran Mishre RR, Berdenis van Berlekom $\mathrm{SH}$ : Het meten van participatie als uitkomst van poliklinische revalidatiebehandeling: Meten is Weten 2; een prospectieve multi-centre studie. [Measurement of participation as an outcome of outpatient rehabilitation: To measure is to know 2; a prospective multi-centre study]. Revalidata 2008, 146:12-16. 
[36] van der Zee CH, Priesterbach AR, van der Dussen L, Kap A, Schepers VP, Visser Meily JM, Post MW: Reproducibility of three self-report participation measures: The ICF Measure of Participation and Activities Screener, the Participation Scale, and the Utrecht Scale for Evaluation of Rehabilitation-Participation. J Rehabil Med 2010, 42:752-757.

[37] Van der Zee CH, Post MW, Hennink J, Schafrat CG, Visser Meily JM, Van Berlekom SB: Validity of the Utrecht Scale for Evaluation of Rehabilitiation. Disabil Rehabil 2012, 34:478-485.

[38] Van der Zee CH, Kap A, Rambaran Mishre RR, Schouten E, Post MW: Responsiveness of four participation measures for outcomes of outpatient rehabilitation. J Rehabil Med 2011, 43:1003-1009.

[39] Vasilaki El, Hosier SG, Cox WM: The efficacy of motivational interviewing as a brief intervention for excessive drinking: a meta-analytic review. Alcohol Alcohol 2006, 41:328-335.

[40] Cohen J: Statistical power analysis for the behavioral sciences. 2nd edn. New Jersey: Lawrence Erlbaum Associates; 1988.

[41] Köke A, Brouwers M, Heuts P, Schiphorst Preuper R, Smeets R, Swaan L, Vlaeyen J, Patijn J: Consensus Rapport Pijnrevalidatie Nederland. Een beschrijvend overzicht van pijnrevalidatieprogramma's in Nederland met betrekking tot doelen en inhoud. [Consensus Report Pain rehabilitation Netherlands. A descriptive overview of pain rehabilitaion programs in the Netherlands with regard to goals and content]. Maastricht: PijnKennis Centrum Maastricht [Pain Knowledge Centre Maastricht]; 2005.

[42] Winter F: De pijn de baas [Mastering pain]. 9th edn. Soest: Ruitenberg Boek; 2008.

[43] Butler D, Moseley L, Sunyata: Explain Pain. Orthopedic Physical Therapy Products; 2003.

[44] Emmons KM, Rollnick S: Motivational interviewing in health care settings. Opportunities and limitations. Am J Prev Med 2001, 20:68-74.

[45] Lane $C_{1}$ Hood $\mathrm{K}$, Rollnick S: Teaching motivational interviewing: Using role play is as effective as using simulated patients. Med Educ 2008, 42:637-644.

[46] Madson MB, Loignon AC, Lane C: Training in motivational interviewing: a systematic review. J Subst Abuse Treat 2009, 36:101-109.

[47] Miller WR, Moyers TB: Eight stages in learning motivational interviewing. J Teach Addict 2007, 5:3-17. 
[48] Miller WR, Yahne CE, Moyers TB, Martinez J, Pirritano M: A randomized trial of methods to help clinicians learn motivational interviewing. J Consult Clin Psychol 2004, 72:1050-1062.

[49] Söderlund LL, Nilsen P, Kristensson M: Learning motivational interviewing: Exploring primary health care nurses' training and counselling experiences. Health Educ J 2008, 67:102-109.

[50] Lansinger B, Nordholm L, Sivik T: Characteristics of low back pain patients who do not complete physiotherapeutic treatment. Scand J Caring Sci 1994, 8:163-167.

[51] Carosella AM, Lackner JM, Feuerstein M: Factors associated with early discharge from a multidisciplinary work rehabilitation program for chronic low back pain. Pain 1994, 57:69-76.

[52] Glombiewski JA, Hartwich-Tersek J, Rief W: Attrition in Cognitive-behavioral Treatment of Chronic Back Pain. Clin J Pain 2010, 26:593-601.

[53] Sloots M, Dekker JH, Pont M, Bartels EA, Geertzen JH, Dekker J: Reasons of drop-out from rehabilitation in patients of Turkish and Moroccan origin with chronic low back pain in The Netherlands: a qualitative study. J Rehabil Med 2010, 42:566-573.

[54] Post MW, van der Zee CH, Hennink J, Schafrat CG, Visser Meily JM, van Berlekom SB: Validity of the Utrecht Scale for Evaluation of Rehabilitation-Participation.

Disabil Rehabil 2011, 34(6):478-85.

[55] Post MW, van der Zee CH, Hennink J, Schafrat CG, Visser Meily JM, van Berlekom SB: Validity of the Utrecht Scale for Evaluation of Rehabilitation-Participation.

Disabil Rehabil 2011, 34(6):478-85.

[56] Pollard CA: Preliminary validity study of the Pain Disability Index. Percept Mot Skills 1984, 59:974.

[57] Chibnall JT, Tait RC: The Pain Disability Index: factor structure and normative data. Arch Phys Med Rehabil 1994, 75:1082-1086.

[58] Carlsson AM: Assessment of chronic pain. I. Aspects of the reliability and validity of the visual analogue scale. Pain 1983, 16:87-101.

[59] Köke A: Rubriek ,Meten in de praktijk' Patiënt specifieke klacht. [Section ,Measurement in practice' Patient specific complaint]. Ned Tijdschr Fysiother 2007, 117:154. 
[60] Beurskens AJ, de Vet HC, Koke AJ, Lindeman E, van der Heijden GJ, Regtop W, Knipschild PG: A patient-specific approach for measuring functional status in low back pain. J Manipulative Physiol Ther 1999, 22:144-148.

[61] Ostelo RW, de Vet HC, Knol DL, van den Brandt PA: 24-item Roland-Morris Disability Questionnaire was preferred out of six functional status questionnaires for post-lumbar disc surgery. J Clin Epidemiol 2004, 57:268-276.

[62] Beurskens AJ, de Vet HC, Koke AJ: Responsiveness of functional status in low back pain: a comparison of different instruments. Pain 1996, 65:71-76.

[63] Spetch LA, Kolt GS: Adherence to sport injury rehabilitation: implications for sports medicine providers and researchers. Phys Ther Sport 2001, 2:80-90.

[64] Schwarzer R, Jerusalem M: Generalized Self-Efficacy scale. In: Measures in health psychology: A user's portfolio Causal and control beliefs. Edited by Weinman J, Wright S, Johnston M. Windsor, UK: Nfer-Nelson; 1995: 35-37

[65] Bosscher RJ, Smit JH: Confirmatory factor analysis of the General Self-Efficacy Scale. Behav Res Ther 1998, 36:339-343.

[66] Anderson KO, Dowds BN, Pelletz RE, Edwards WT, Peeters-Asdourian C: Development and initial validation of a scale to measure self-efficacy beliefs in patients with chronic pain. Pain 1995, 63:77-84.

[67] Hakkaart-van Roijen L, van Straten A, Donker M, Tiemens B: Manual Trimbos/iMTA questionnaire for Costs associated with Psychiatric illness (TiC-P). Rotterdam: Institute for Medical Technology Assessment, Erasmus University Rotterdam; 2002.

[68] Hakkaart-van Roijen L, Bouwmans CAM: Short Form-Health and Labour Questionnaire (SF-HLO). Rotterdam: Institute for Medical Technology Assessment. Erasmus Universitair Medisch Centrum Rotterdam; 2007.

[69] Hakkaart- van Roijen L, Tan SS, Bouwmans CAM: Handleiding voor kostenonderzoek, methoden en standaard kostprijzen voor economische evaluaties in de gezondheidszorg [Manual for research of costs, methods and standard prices for economic evaluations in health care]. Diemen, the Netherlands: College voor Zorgverzekeringen (CVZ) [Dutch Health Care Insurance Board (CVZ)]; Geactualiseerde versie 2010. [Updated version 2010].

[70] Oostenbrink JB, Koopmanschap MA, Rutten FF: Standardisation of costs: the Dutch Manual for Costing in economic evaluations. Pharmacoeco 2002, 20:443-454. 
[71] van der Zee KI, Sanderman R: Het meten van de algemene gezondheidstoestand met de RAND-36. Een handleiding [Measuring the General Health Status by the RAND-36. A manual]. Groningen: Noordelijk Centrum voor Gezondheidsvraagstukken (NCG) [Northern centre for health issues (NCG)]; 1993.

[72] Goossens ME, Evers SM, Vlaeyen JW, Rutten-van Mölken MP, van der Linden SM: Principles of economic evaluation for interventions of chronic musculoskeletal pain. Eur J Pain 1999, 3:343-353.

[73] Brazier J, Roberts J, Deverill M: The estimation of a preference-based measure of health from the SF-36. J Health Econ 2002, 21:271-292.

[74] Dolan P: Modeling Valuations for Euro0ol Health States. Med Care 1997, 35:1095-1108.

[75] Moyers TB, Martin T, Christopher PJ, Houck JM, Tonigan JS, Amrhein PC: Client language as a mediator of motivational interviewing efficacy: where is the evidence? Alcohol Clin Exp Res 2007, $31: 40$ s-47s.

[76] Moyers TB, Miller WR, Hendrickson SM: How does motivational interviewing work? Therapist interpersonal skill predicts client involvement within motivational interviewing sessions. J Consult Clin Psychol 2005, 73:590-598.

[77] Nielson WR, Jensen MP, Kerns RD: Initial development and validation of a multidimensional pain readiness to change questionnaire. J Pain 2003, 4:148-158.

[78] Nielson WR, Armstrong JM, Jensen MP, Kerns RD: Two brief versions of the multidimensional pain readiness to change questionnaire, version 2 (MPRCQ2). Clin J Pain 2009, 25:48-57.

[79] Vlaeyen JW, Morley S: Cognitive-behavioral treatments for chronic pain: what works for whom? Clin J Pain 2005, 21:1-8.

[80] Smeets RJ, Beelen S, Goossens ME, Schouten EG, Knottnerus JA, Vlaeyen JW: Treatment expectancy and credibility are associated with the outcome of both physical and cognitive-behavioral treatment in chronic low back pain. Clin J Pain 2008, 24:305-315.

[81] Goossens ME, Vlaeyen JW, Hidding A, Kole-Snijders A, Evers SM: Treatment expectancy affects the outcome of cognitive-behavioral interventions in chronic pain. Clin J Pain 2005, 21:18-26; discussion 69-72. 
[82] Devilly GJ, Borkovec TD: Psychometric properties of the credibility/expectancy questionnaire. J Behav Ther Exp Psychiatry 2000, 31:73-86.

[83] Ryan RM, Plant RW, O'Malley S: Initial motivations for alcohol treatment: Relations with patient characteristics, treatment involvement, and dropout. Addict Behav 1995, 20:279-297.

[84] Beck AT, Ward CH, Mendelsohn M, Mock J, Erbaugh J: An inventory for measuring depression. Arch Gen Psychiatry 1961, 4:561-571.

[85] Beck AT, Steer RA, Carbin MG: Psychometric properties of the Beck Depression Inventory: Twenty-five years of evaluation. Clin Psychol Rev 1988, 8:77-100.

[86] Beck AT, Steer RA: Beck Depression Inventory. San Antonio: Psychological Corporation; 1993.

[87] McCracken LM, Zhao-O'Brien J: General psychological acceptance and chronic pain: There is more to accept than the pain itself. Eur J Pain 2010, 14:170-175.

[88] Bond FW, Hayes SC, Baer RA, Carpenter KM, Guenole N, Orcutt HK, Waltz T, Zettle RD: Preliminary psychometric properties of the Acceptance and Action Questionnaire-II: A revised measure of ssychological inflexibility and experiential avoidance. Behav Ther 2011, 42:676-688.

[89] Jacobs N, Kleen M, De Groot F, A-Tjak J: Het meten van experiëntiële vermijding. De Nederlandse versie van de Acceptance and Action Qustionnaire (AAQ-II). [Measurement of experiental avoidance. The Dutch version of the Acceptance and Action Questionnaire (AAQ-II)]. Gedragstherapie 2008, 41:349-361.

[90] Goossens ME, Kindermans HP, Morley SJ, Roelofs J, Verbunt J, Vlaeyen JW: Self-discrepancies in work-related upper extremity pain: relation to emotions and flexible-goal adjustment. Eur J Pain 2010, 14:764-770.

[91] Kranz D, Bollinger A, Nilges P: Chronic pain acceptance and affective well-being: A coping perspective. Eur J Pain 2010, 14:1021-1025.

[92] Brandtstädter J, Renner G: Tenacious goal pursuit and flexible goal adjustment: explication and age-related analysis of assimilative and accommodative strategies of coping. Psychol Aging 1990, 5:58-67.

[93] Rollnick S, Butler CC, Kinnersley P, Gregory J, Mash B: Motivational interviewing BMJ 2010, 340:c1900. 
[94] Revised Global Scales: Motivational Interviewing Treatment Integrity 3.1.1 (MITI 3.1.1) [http://casaa.unm.edu/download/MITI3_1.pdf]

[95] Moyers TB, Martin T, Manuel JK, Hendrickson SM, Miller WR: Assessing competence in the use of motivational interviewing. J Subst Abuse Treat 2005, 28:19-26.

[96] Pierson HM, Hayes SC, Gifford EV, Roget N, Padilla M, Bissett R, Berry K, Kohlenberg B, Rhode R, Fisher G: An examination of the Motivational Interviewing Treatment Integrity code. J Subst Abuse Treat 2007, 32:11-17.

[97] Forsberg L, Berman AH, Kallmen $H_{1}$ Hermansson U, Helgason AR: A test of the validity of the Motivational Interviewing Treatment Integrity Code. Cogn Behav Ther 2008, 37:1-9.

[98] Madson MB, Campbell TC: Measures of fidelity in motivational enhancement: a systematic review. J Subst Abuse Treat 2006, 31:67-73.

[99] Miller WR, Mount KA: A small study of training in Motivational Interviewing: does one workshop change clinician and client behavior?. Behav Cogn Psychoth 2001, 29:457-471.

[100] Moyers T, Martin T, Catley D, Harris KJ, Ahluwalia JS: Assessing the integrity of motivational interviewing interventions: Reliability of the motivational interviewing skills code. Behav and Cogn Psychoth 2003, 31:177-184.

[101] Steckler A, Linnan L: Process evaluation for public health interventions and research. San Francisco, CA Jossey-Bass; 2002.

[102] de Witte L, Schoot T, Proot I: Development of the client-centred care questionnaire. J Adv Nurs 2006, 56:62-68.

[103] Schoot T, Friesen J, Balk S, Köke A: Implementatie van cliëntgecentreerde zorg binnen het multidisciplinaire pijnteam van Adelante Zorggroep te Hoensbroek [Implementation of client-centred care at the multidisciplinary pain team of the Adelante care group in Hoensbroek.]. 1-10-2010 edn. Hoensbroek, the Netherlands: Adelante 2010.

[104] Fenwick E, O'Brien BJ, Briggs A: Cost-effectiveness acceptability curves - facts, fallacies and frequently asked questions. Health Econ 2004,13:405-415.

[105] Steiner WA, Ryser L, Huber E, Uebelhart D, Aeschlimann A, Stucki G: Use of the ICF model as a clinical problem-solving tool in physical therapy and rehabilitation medicine.

Phys Ther 2002, 82:1098-1107. 
[106] Stucki G, Ewert T, Cieza A: Value and application of the ICF in rehabilitation medicine. Disabil Rehabil 2003, 25:628-634.

[107] Dworkin RH, Turk DC, Wyrwich KW, Beaton D, Cleeland CS, Farrar JT, Haythornthwaite JA, Jensen MP, Kerns RD, Ader DN, et al: Interpreting the clinical importance of treatment outcomes in chronic pain clinical trials: IMMPACT recommendations. J Pain 2008, 9:105-121.

[108] Dworkin RH, Turk DC, McDermott MP, Peirce-Sandner S, Burke LB, Cowan P, Farrar JT, Hertz S, Raja SN, Rappaport BA, et al: Interpreting the clinical importance of group differences in chronic pain clinical trials: IMMPACT recommendations. Pain 2009, 146:238-244.

[109] Leonhardt C, Keller S, Chenot JF, Luckmann J, Basler HD, Wegscheider K, Baum E, Donner-Banzhoff N, Pfingsten M, Hildebrandt J, et al: TTM-based motivational counselling does not increase physical activity of low back pain patients in a primary care setting A cluster-randomized controlled trial. Patient Educ Couns 2008, 70:50-60.

[110] Walters ST, Matson SA, Baer JS, Ziedonis DM: Effectiveness of workshop training for psychosocial addiction treatments: a systematic review. J Subst Abuse Treat 2005, 29:283-293.

[111] Söderlund LL, Madson MB, Rubak S, Nilsen P: A systematic review of motivational interviewing training for general health care practitioners. Patient Educ Couns 2011, 84:16-26. 


\section{Chapter 3}

Treatment fidelity of a nurse-led Motivational Interviewing-based pre-treatment in pain rehabilitation

Mertens, V.-C.

Forsberg, L.

Verbunt, J. A.

Smeets, R. J.

Goossens, M. E.

Behavioral Health Services \& Research. 2015.

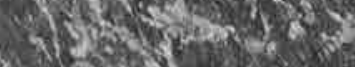

asting

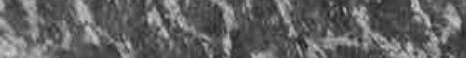

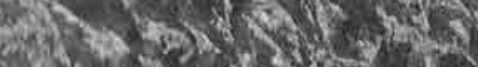

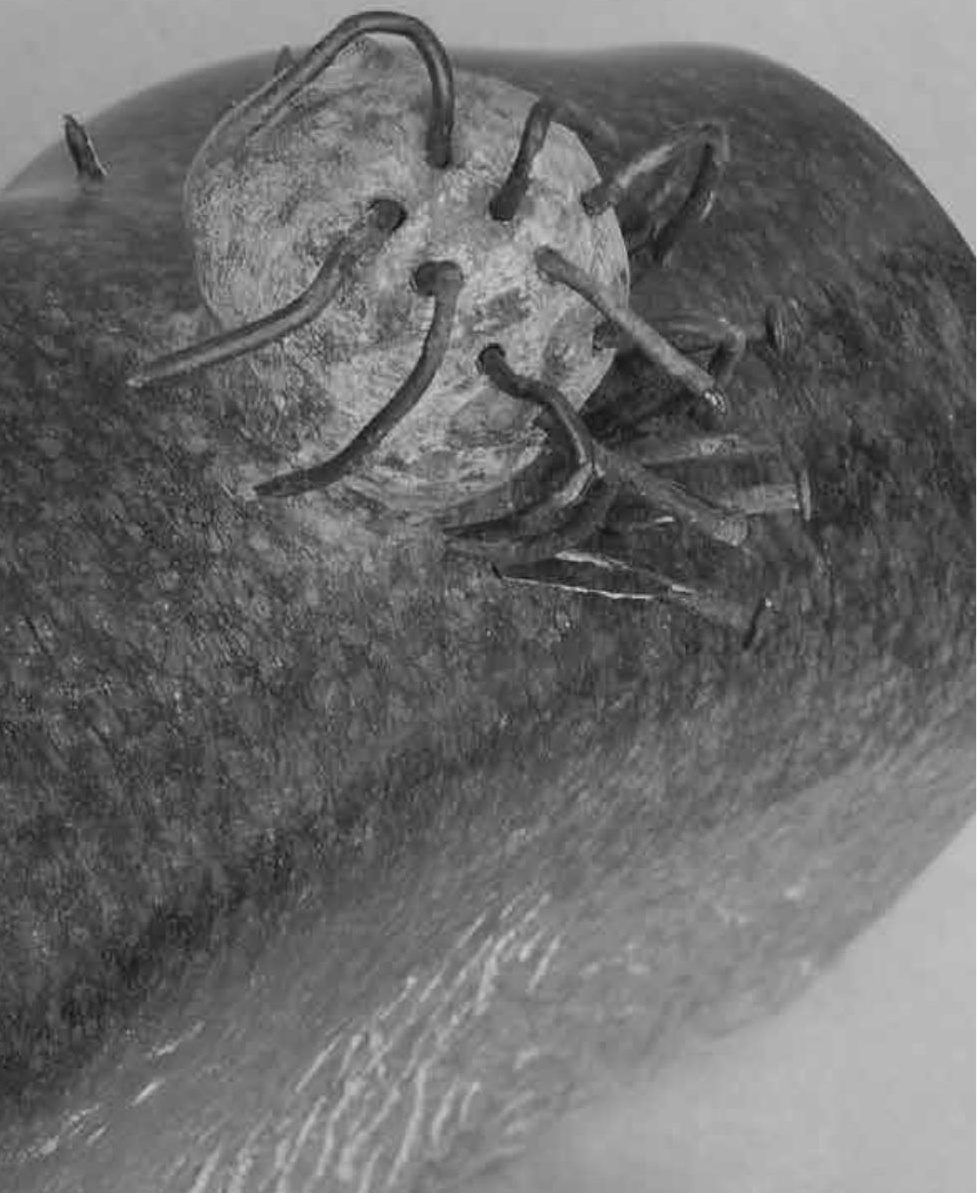




\section{Abstract}

Treatment fidelity and proficiency of a nurse-led Motivational Interviewing (MI)-based pretreatment and control condition was evaluated. A random sample was scored by means of the Motivational Interviewing treatment Integrity (MITI) Scale, and a second rater was in charge. MI fidelity was satisfactory for three out of five ratings. Most mean ratings were higher in the MI-based intervention, however, differences were not statistically significant. The threshold for beginning Ml-proficiency was only exceeded for one score and one additional measure. In general higher levels of fidelity in the intervention condition confirmed that MI was partially applied there. Although the quality of MI delivery as well as mixed inter-rater reliabilities of the fidelity scores leaves room for improvement, robust findings between the two raters were found. These results suggest the need for rigor selection of Ml-counselors on beforehand, and continuous supervision. Furthermore, fidelity check in studies using $\mathrm{MI}$ is needed.

\section{Introduction}

Motivational interviewing (MI) is a person-centered form of counseling to elicit and strengthen motivation for change [1]. Motivation and adherence challenges are not unique to addiction treatment, wherein the historical roots of MI lay, but MI is also promising for other applications such as to promote treatment adherence [2]. Several systematic reviews and meta-analyses showed the effectiveness of Motivational interviewing across behaviors and contexts in health care [3-6]. The effect of MI as pre-treatment has specifically been acknowledged $[3,4,7]$. Positive effects in pain rehabilitation treatment [8-11], and moderate quality evidence has been provided for its successful application to promote physical activity in people with chronic health conditions [12].

\section{MI has two components:}

1) The relational component consists of the so-called 'MI-spirit', a counselor-attitude characterized by genuine interest in the client and empathy;

2) The technical component consists of techniques to evocate, elicit, and reinforce change talk [13] (by e.g. the use of open questions and reflections). However, so far, MI has mostly been emphasized as a spirit rather than a technique [14]. At first sight, MI-principles -asking open questions, 
giving reflections- look simple. But the underlying principles resulting in Ml-spirit like empathy are a complex mix of skills that take considerable time to learn [1]. As a consequence, the quality of MI delivery can vary tremendously. Since the quality of MI delivered is an important factor for the beneficial effect, this diversity can have a huge impact [15]. For this reason, it is very important to check and accurately test whether $\mathrm{MI}$ is delivered as intended $[16,17]$. Or in other words, to check its treatment fidelity (sometimes referred to as treatment integrity).

This can have important implications for the conclusion drawn regarding effectiveness. E.g. results could be related to something else than the hypothesized working mechanism of the MI intervention because of non-adherence to procedures or failing therapist competence.

From a few other studies it is known, that Ml-fidelity measures have predictive validity to predict patient behavior following MI treatment [18-20]. Additionally, in terms of therapists' competence, Ml-training is related to $\mathrm{Ml}$ - fidelity by suppressing $\mathrm{Ml}$-countering responses related to resistance and poorer outcome which has been linked to increased change talk which in turn predicts behavior change in $\mathrm{MI}$ [20].

As $\mathrm{MI}$-practitioners seem to overestimate their functioning, self-report of MI-fidelity can be seen as unreliable $[21,22]$. Furthermore, fidelity measures can serve as manipulation check to discriminate MI reliably from non-MI-based control interventions [23]. Thus, quality assurance based upon recordings of the actual sessions and the usage of MI-specific coding instruments can estimate whether MI was actually delivered.

However, regardless this hypothesized impact of variety in MI quality delivered, only 17\% of research specifically within the field of MI research assessed fidelity adequately [5]. In the domain somatoform disorders and of research in chronic pain specifically, two of the four studies using $\mathrm{MI}$ checked for treatment fidelity $[8,9]$, two other studies did not $[10,11]$.

Aim of the present study is to evaluate the treatment fidelity of an MI-based intervention and an educational control treatment in pain rehabilitation. It is hypothesized that MI-proficiency will be higher in the intervention condition and that the intervention condition can be distinguished from a non-MI based educational control condition. 


\section{Methods}

This study is part of a large two-armed randomized controlled trial (RCT) (a detailed description of the RCT is given in detail elsewhere) [24]. This study assessed the effectiveness of a nurse-led MI-based pre-treatment compared to an attention-control pre-treatment pain education in a Dutch chronic musculoskeletal pain population before the start of the actual pain rehabilitation treatment.

In figure 1 an overview of the content of both study conditions is given [24].

\begin{tabular}{|c|c|c|}
\hline & $\begin{array}{l}\text { Motivational interviewing } \\
\text { based pre-treatment (MIP) }\end{array}$ & $\begin{array}{l}\text { Pain education pre-treatment } \\
\text { (Usual Care, UC) }\end{array}$ \\
\hline Goal & $\begin{array}{l}\text { Explore participant's life situati- } \\
\text { on, impairments and ambivalen- } \\
\text { ces in order to enhance intrinsic } \\
\text { motivation }\end{array}$ & $\begin{array}{l}\text { Provide the participant with } \\
\text { information. }\end{array}$ \\
\hline Basic principle & Motivational Interviewing & $\begin{array}{l}\text { Health education and patient } \\
\text { education. }\end{array}$ \\
\hline Foundation & $\begin{array}{l}\text { Content based on patient-dri- } \\
\text { ven topics derived from the } \\
\text { ICF-model }\end{array}$ & $\begin{array}{l}\text { Content based on the book } \\
\text { 'De pijn de baas' (Winter 2008) } \\
\text { (Mastering pain). }\end{array}$ \\
\hline Protocol rigidity & $\begin{array}{l}\text { Content sessions tailored to the } \\
\text { patients' readiness to change }\end{array}$ & $\begin{array}{l}\text { Content sessions is fixed by me- } \\
\text { ans of the treatment protocol }\end{array}$ \\
\hline \multirow[t]{2}{*}{ Content $1^{\text {st }}$ session } & $\begin{array}{l}\text { Exploration actual (life) situation, } \\
\text { burden and impairments of the } \\
\text { chronic pain in daily life, }\end{array}$ & $\begin{array}{l}\text { Provision of general health } \\
\text { education about topics related } \\
\text { to chronic pain }\end{array}$ \\
\hline & $\begin{array}{l}\text { Assessing + enhancing motiva- } \\
\text { tion, self-efficacy, and readiness } \\
\text { to change for behavior }\end{array}$ & $\begin{array}{l}\text { Provision of information } \\
\text { regarding core elements of pain } \\
\text { rehabilitation }\end{array}$ \\
\hline Content $2^{\text {nd }}$ session & $\begin{array}{l}\text { Giving feedback adapted to the } \\
\text { state of readiness-to-change. } \\
\text { Continuation exploration. } \\
\text { Continuation assessing +enhan- } \\
\text { cing motivation, self-efficacy, } \\
\text { and readiness to change for } \\
\text { behavior }\end{array}$ & $\begin{array}{l}\text { Continuation of general health } \\
\text { education about topics related } \\
\text { to chronic pain }\end{array}$ \\
\hline Feedback & $\begin{array}{l}\text { Is given related to the stage of } \\
\text { change of the participant accor- } \\
\text { ding to the MI-principles. }\end{array}$ & Is not given. \\
\hline
\end{tabular}

Figure 1: Main features of the two interventions 


\section{Training of the nurses}

Nurses provided one treatment condition only and were trained specifically for the intervention they had to deliver.

Training for the intervention (Motivational interviewing based pre-treatment (MIP)): In the MIP condition, the nurses were both experienced MI coaches. In two half-day sessions the nurses' $\mathrm{MI-knowledge} \mathrm{and} \mathrm{experience} \mathrm{in} \mathrm{the} \mathrm{context} \mathrm{of} \mathrm{chronic} \mathrm{pain} \mathrm{rehabilitation} \mathrm{was} \mathrm{updated} \mathrm{based}$ on an evidence based Ml- training tailored to their specific needs. The training was provided by a certified MI-trainer. Follow up training during the trial consisted of regular supervision (three times half a day during the trial period of 1,5 years). The training was based upon actual cases and by providing direct feedback on audio taped MIP sessions by the same MI-trainer.

Training for the control condition: The two nurses of the pain education control condition were experienced in the field of (pain) rehabilitation and received a three-hour refresher training in communication skills and general principles of health education. In addition, the content of relevant chapters of the book 'Mastering pain' was discussed. Follow up training included two sessions lasting two hours in which problems encountered were discussed.

\section{Measurement instrument: MITI and procedures of scoring}

To test the treatment integrity the Motivational Interviewing Treatment Integrity (MITI version 3.1.1) Scale was used [25]. This scale has shown to be reliable $[23,26-28]$ and valid $[25,28]$. The MITI focuses exclusively on therapist's functioning [23].

The MITI assessment instrument is composed of two different parts: 'Global Counselor Ratings' and 'Behavior Counts' (see figure 2). Both were evaluated and rated during the preselected twenty-minute long session sample in two separated rounds. Coding in the first round was performed without interruption. In the second round, each utterance was categorized in one of the five counselor-related Behavior Counts, and the total frequency of each specific behavior such as 'Giving Information' was counted [28]. After those two rounds of rating, five Summary Scores were calculated and compared to existing thresholds to evaluate fidelity finally (see end of this section).

\section{First round: Global Counselor Ratings}

The MITI's Global Counselor Ratings were designed to capture the rater's overall impression of the session and cover five aspects:

1. Evocation,

2. Collaboration,

3. Autonomy/Support,

4. Direction, and

5. Empathy.

A 5-point scale ranging from 1 (low) to 5 (high) has to be scored by the assessor. A precise definition of each variable can be found in the MITI's manual [25]. 


\section{Motivational Interviewing Treatment Integrity Code (MITI) Coding Sheet}

\begin{tabular}{|c|c|c|c|c|c|c|}
\hline \multirow[b]{2}{*}{ Evocation } & \multirow{2}{*}{$\begin{array}{l}\text { Abbreviation } \\
\text { (E) }\end{array}$} & \multicolumn{5}{|c|}{ Rating } \\
\hline & & $\begin{array}{l}1 \\
\text { low }\end{array}$ & 2 & 3 & 4 & $\begin{array}{l}5 \\
\text { high }\end{array}$ \\
\hline Collaboration & (C) & $\begin{array}{l}1 \\
\text { low }\end{array}$ & 2 & 3 & 4 & $\begin{array}{l}5 \\
\text { high }\end{array}$ \\
\hline Autonomy / Support & $(A / S)$ & $\begin{array}{l}1 \\
\text { low }\end{array}$ & 2 & 3 & 4 & $\begin{array}{l}5 \\
\text { high }\end{array}$ \\
\hline Direction & & $\begin{array}{l}1 \\
\text { low }\end{array}$ & 2 & 3 & 4 & $\begin{array}{l}5 \\
\text { high }\end{array}$ \\
\hline Empathy & & $\begin{array}{l}1 \\
\text { low }\end{array}$ & 2 & 3 & 4 & $\begin{array}{l}5 \\
\text { high }\end{array}$ \\
\hline
\end{tabular}

\begin{tabular}{|l|l|l|}
\hline \multicolumn{2}{|l|}{$2^{\text {nd }}$ Round: Behaviour Counts } & Nr. of Counts \\
\hline Giving Information & Giving Information (GI) & \\
\hline MI Adherent & $\begin{array}{l}\text { Asking permission, affirm, emphasize } \\
\text { control, support (MIA) }\end{array}$ \\
\hline MI Non-Adherent & Advise, confront, direkt (MINA) & \\
\hline Question & Closed Questions (CO) & \\
\hline & Open Questions (O0) & \\
\hline Reflection & Total Questions (CQ + O0) & \\
\hline & Simple Reflection (SR) & \\
\hline & Complex Reflection (CR) & \\
\hline & Total Reflections (SR + CR) & \\
\hline
\end{tabular}

\section{MITI Summary Scores and belonging thresholds}

\begin{tabular}{|l|l|l|}
\hline & Calculation & Threshold beginning proficiency \\
\hline Global Spirit & $(\mathrm{E}+\mathrm{C}+\mathrm{A} / \mathrm{S}) / 3$ & $\emptyset 3,5$ \\
\hline \% Open Questions & $\mathrm{OQ} /(\mathrm{OQ}+\mathrm{CQ})$ & $50 \%$ \\
\hline \% Complex Reflections & CR / Total Reflections & 1 \\
\hline Reflections Questions-ratio & Total Reflections / Total Questions & $40 \%$ \\
\hline$\%$ MI Adherent & MIA / (MIA + MINA) & $90 \%$ \\
\hline
\end{tabular}

Two additional Ml-fidelity measures and belonging thresholds

\begin{tabular}{|l|l|l|}
\hline & Derived from & Threshold \\
\hline Empathy & Global Counselor Ratings & $\emptyset 3,5$ \\
\hline MI Non-adherent & Behaviour Count & As low as possible \\
\hline
\end{tabular}

Figure 2: The MITI coding form 


\section{Second round: Behavior Counts}

Next the assessor counted the total frequency of 5 categories of verbal behavior:

1. Giving information,

2. MI-adherent responses (i.e. asking permission, emphasizing control, affirming or supporting),

3. $\mathrm{MI}$ non-adherent responses (i.e. advising without permission, confronting or directing),

4. Question (open vs. closed), and

5. Reflection (simple vs. complex) [25].

\section{MITI Summary Scores and belonging thresholds for beginning proficiency}

After the two rounds five indices (MITI Summary Scores) were calculated to evaluate MI-fidelity. One is gained from the Global Counselor Ratings: Ml-spirit and four were gained from the Behavior Counts:

1. Percentage of Open questions,

2. Percentage of Complex reflections,

3. Percentage of MI-adherent responses, and

4. A Reflections-to-questions ratio.

After this, the Summary Scores were checked against the thresholds score for 'beginning proficiency' level [25]. This threshold was defined as follows: 'Beginning proficiency' needs at least 3.5 points (out of 5) for the global score Spirit, and a 'Reflection to Question ratio' of 1. The 'percentage of Open Questions' and 'Complex Reflections' had to be at least 50\% and 40\% respectively. And finally, the 'percentage of MI-adherent behaviors' had to be $90 \%$.

\section{Two additional MI-fidelity measures and belonging thresholds}

Furthermore, as Empathy and MI non-adherent behaviors are specifically mentioned as predictors for successful treatment $[20,29]$, both were considered of such importance that they were also taken into account in the evaluation of MI fidelity. This was done by checking whether Empathy crossed the threshold of the other Global Counselor Ratings (namely 3.5), and counting the amount of $\mathrm{MI}$ non-adherent responses (being as low as possible as avoiding $\mathrm{MI}$ non-adherent responses might be more important than using $\mathrm{Ml}$-adherent responses) [25].

\section{Procedures of the sampling}

All sessions (intervention and control condition) were audio taped, and a random sample of $20 \%(n=64)$ was used to test treatment fidelity. To collect this sample, randomization of audio taped samples was stratified for first and second session as well as for intervention and control condition. In case the actual audiotape was not available due to non-consent of the participant to record $(n=4)$ or technical problems $(n=12)$, the consecutive tape of the next participant was chosen. A research assistant selected the 20 minutes session.

All the selected samples were scored by the first rater (VCM), half of these recordings ( $n=32$ ) were also scored by a second rater $(\mathrm{JJ})$ blinded for group allocation. 


\section{Ethics}

The study was approved by the Medical Ethical the University Hospital Maastricht and Maastricht University. The study is registered in a public trial registry (Nederlands Trial Register NTR 3065). All participants provided written informed consent for as well study participation as well as audio recording of the sessions.

\section{Training of the MITI raters}

Before the start of the study, both raters received initially a 40 hours training program in MITI coding according to Moyers et al. (2010) [25]. Furthermore, both raters were re-trained at the start of the coding work by using English-spoken training materials from the Center for Alcoholism, Substance Abuse and Addictions (CASAA, University of New Mexico) and worked under supervision of an employee of one of the existing MI coding labs, MIC lab, Karolinska Institutet, Stockholm, Sweden.

Furthermore, the first six double-coded sessions served as training material. Reliability of both raters was stated two times: 1) Before the start of the actual rating by calculating ICCs between the two raters and the coding lab's rating who served as 'gold standard' on similar English spoken training materials. 2) During the actual rating, reliability was stated also (see section Statistical analysis).

During the period of rating, the first rater (VCM) participated in weekly intervision of the coding lab and could furthermore consult experienced raters. Thirty two sessions (19 intervention condition, 13 control condition) were independently scored by the second rater (JJ).

\section{Scoring double coded sessions}

For the double-coded sessions the raters scored the sessions independently and also had to reach consensus for the Global Counselor Ratings afterwards. To score the Behavior Counts the arithmetic mean was calculated. In the following this is referred to as consensus approach.

\section{Statistical analysis}

To evaluate MI-quality in both study conditions, scores on all MITI domains were interpreted according the manual and belonging thresholds [25], and the two additional Ml-fidelity measures. To test for differences in MI fidelity scores between conditions, an independent t-test (significance level of $>$.05) was used. In case of non-normal distribution of either sample of the first or the second rater, the Mann-Whitney test was used.

Interrater reliability was calculated based on the intraclass correlation coefficients (ICCS) [30] by means of a 2-way mixed effects ICC model (absolute agreement) and interpreting single measures in the SPSS output. Inter rater reliability was classified according Cicchetti and Sparrow (1981) who indicated ICC $<0.40$ as poor, $0.40-0.59$ as fair, $0.60-0.74$ as good, and $0.75-1.00$ as excellent [30]. Furthermore, in case of an ordinal scale (present in the Global Counselor Ratings) Krippendorff's alpha (KALPHA) [31] was also calculated by using a macro [32].

Post hoc comparisons took place on nurse-level in order to get more insight in nurse-specific fidelity. 
Data were analyzed using Statistical Software Package for Social Sciences (SPSS), version 21 (SPSS Inc., Chicago, IL).

Reliability/ Sensitivity analysis

Sensitivity analysis consisted of two subsequent steps:

1. Checking inter-rater reliability between the results of the double-coded sessions and,

2. comparing the results of two approaches of the ratings: Ratings of the first rater with the second rater as well as a consensus approach between the two raters.

\section{Results}

The four nurses of both conditions participating in this study were experienced in the working field of (pain) rehabilitation. The two nurses of the MI-based intervention condition had four years of experience with $\mathrm{Ml}$; the two nurses of the educational control condition had experience with patient education in rehabilitation care.

A random sample $n=64$ of all nurse-led sessions ( $n=37$ intervention condition, $n=27$ control condition) was scored by the first rater (VCM). Out of this sample, $n=26$ sessions ( 18 intervention condition, 8 control condition) were also scored by a second rater $(\mathrm{JJ}) .6$ sessions were used as training material at the start of the double coding.

\section{Overall results}

\section{Global Counselor Ratings}

According to the MITI, the nurses' beginning proficiency competence in the use of MI was satisfactory for the Global Counselor Ratings Direction (intervention as well as control condition), and Empathy and Evocation (intervention condition only).

Table 1 shows that all mean Global Counselor Ratings were, except for Direction (.18), higher (between 0.34 and 1.97 points higher on a 5 point Likert scale) in the Ml-based intervention condition compared to the education control condition. However, mean differences were not statistically significant different. 
Table 1 Comparison of the means for the MITI global scores in educational control condition and Ml-based intervention condition of the investigated study

\begin{tabular}{|c|c|c|c|c|c|c|c|}
\hline Criterion & $\begin{array}{c}\text { Mean } \\
\text { control } \\
\text { condition } \\
(n=27)\end{array}$ & $\begin{array}{c}\text { Mean in- } \\
\text { tervention } \\
\text { condition } \\
(n=37)\end{array}$ & $\begin{array}{l}\text { MITI 3.1.1 } \\
\text { threshold } \\
\text { (begin- } \\
\text { ning pro- } \\
\text { ficiency) }\end{array}$ & $\begin{array}{l}\text { Threshold } \\
\text { exceede- } \\
\text { d?c }\end{array}$ & $\begin{array}{c}\text { Mann- } \\
\text { Whit-ney } \\
\text { U }\end{array}$ & z & $\begin{array}{l}\mathrm{p} \text { (two- } \\
\text { tailed) }\end{array}$ \\
\hline \multicolumn{8}{|c|}{ Global Counselor Ratings (range 1-5) } \\
\hline Evocation & 1.65 & 3.62 & 3.5 & + & 421.00 & -0.93 & 0.34 \\
\hline Collaboration & 1.59 & 3.43 & 3.5 & - & 438.50 & -0.87 & 0.39 \\
\hline Autonomy/ Support & 2.85 & 3.19 & 3.5 & - & 418.50 & -0.17 & 0.10 \\
\hline Direction & 4.58 & 4.49 & 3.5 & ++ & 404.50 & -1.38 & 0.18 \\
\hline Empathy & 2.67 & 3.95 & 3.5 & + & 410.00 & -1.12 & 0.18 \\
\hline \multicolumn{8}{|l|}{ Behavior Counts } \\
\hline $\begin{array}{l}\text { \# Giving } \\
\text { Information }^{a}\end{array}$ & 17.81 & 16.86 & - & $\mathrm{n} / \mathrm{a}$ & -0.447 & 62 & 0.65 \\
\hline \# Ml-adh. responses & 1.15 & 0.59 & - & $\mathrm{n} / \mathrm{a}$ & 446.00 & -0.79 & 0.43 \\
\hline $\begin{array}{l}\text { \# Ml-nonadh } \\
\text { responses }\end{array}$ & 2.37 & 1.24 & - & $\mathrm{n} / \mathrm{a}$ & 471.00 & -0.40 & 0.69 \\
\hline \# Closed Questions & 5.56 & 13.89 & - & $\mathrm{n} / \mathrm{a}$ & 6.34 & 62 & 0.00 \\
\hline \# Open Questions & 2.30 & 10.62 & - & $\mathrm{n} / \mathrm{a}$ & 488.00 & -0.16 & 0.87 \\
\hline Total Questions ${ }^{\mathrm{a}}$ & 7.85 & 24.51 & - & $\mathrm{n} / \mathrm{a}$ & 7.99 & 62 & 0.00 \\
\hline \# Simple Reflections ${ }^{a}$ & 7.78 & 16.89 & - & $\mathrm{n} / \mathrm{a}$ & 6.50 & 55.27 & 0.00 \\
\hline $\begin{array}{l}\text { \#Complex } \\
\text { Reflections }^{\mathrm{a}}\end{array}$ & 2.93 & 4.84 & - & $\mathrm{n} / \mathrm{a}$ & 2.51 & 62 & 0.01 \\
\hline Total Reflections ${ }^{\mathrm{a}}$ & 10.70 & 21.73 & - & $\mathrm{n} / \mathrm{a}$ & 5.89 & 62 & 0.00 \\
\hline \multicolumn{8}{|c|}{ Summary Scores and belonging thresholds } \\
\hline Spirit & 2.01 & 3.41 & 3.5 & - & 394.50 & -1.29 & 0.20 \\
\hline$\%$ Open Questions & 35.66 & 40.09 & 50 & - & 447.00 & -0.54 & 0.59 \\
\hline $\begin{array}{l}\text { \% Complex } \\
\text { Reflections }^{\mathrm{a}}\end{array}$ & 23.01 & 22.72 & 40 & - & -0.17 & 44.49 & 0.86 \\
\hline $\begin{array}{l}\text { Reflections- } \\
\text { questions ratio }\end{array}$ & 3.53 & 1.01 & 1 & ++ & 464.50 & -0.30 & 0.76 \\
\hline \% Ml-adh. responses & 33.70 & 37.65 & 90 & - & 223.50 & -0.75 & 0.45 \\
\hline \multicolumn{8}{|c|}{ Two additional MI-fidelity measures and belonging thresholds } \\
\hline Empathy & 2.67 & 3.95 & 3.5 & + & 410.00 & -1.12 & 0.18 \\
\hline $\begin{array}{l}\text { \# MI-nonadh } \\
\text { responses }\end{array}$ & 2.37 & 1.24 & - & + & 471.00 & -0.40 & 0.69 \\
\hline
\end{tabular}

${ }^{a}$ For those ratings, assumptions for parametric testing (indep. t-test) are fulfilled, therefore mean, $t$ and $d f$ are presented in the subsequent cells; ${ }^{c}+:$ Threshold exceeded for one condition; ${ }^{c}++:$ for both conditions 


\section{Behavior Counts}

The Ml-based intervention condition had statistically significant higher scores for the amount of Closed Questions, Total questions, Simple reflections, Complex reflections and the amount of Total reflections.

\section{Summary Scores and belonging thresholds of beginning proficiency}

In terms of MI competence according to the MITI, the threshold for beginning proficiency was exceeded for the Reflections-to-questions ratio only (intervention as well as control condition). The Percentage of Open Questions and the Percentage of Ml-adherent responses showed slightly higher mean ratings in the intervention condition compared to the control condition (40.09\% and $35.66 \%$, respectively by a threshold of $50 \%$, and $37.65 \%$ and $33.70 \%$, respectively by a threshold of 90\%).

The Percentage of Complex reflections (23.01\% and $22.72 \%$, respectively by a threshold of $40 \%$ ) as well as Reflections-to-questions ratio (3.53 and 1.01 respectively) were higher in the control condition compared to the Ml-based intervention condition.

\section{Two additional MI-fidelity measures and belonging thresholds}

Empathy was scored higher, but not statistically different in the MI-based intervention condition and the threshold for beginning competence was reached for the Ml-based intervention.

One time less $\mathrm{MI}$ non-adherent responses in the intervention condition compared to the control condition were found (2.37, and 1.24 respectively).

\section{Reliability/ Sensitivity analysis}

Quality of reliability between the two raters was mixed (see table 2), ranging from poor up to excellent.

The ratings of the second rater as well as the consensus approach of both raters together confirmed the previously mentioned findings of the first rater. This led to the overall conclusion that all ratings for Global Counselor Ratings, and five out of the seven Behavior Count (sub)scores, were in terms of Ml-fidelity higher in the intervention condition compared to the control condition. Similarly, it was found that not all thresholds for beginning Ml-proficiency were exceeded. Only in the consensus approach a statically significant difference for the Global Counselor Rating Evocation $(<0.01)$ between the intervention and control condition was found. However, in no condition the score did exceed the threshold in ratings of both raters.

In the consensus approach, intervention condition and control condition can be clearly discriminated for global scores Spirit $(<0.01)$ and Collaboration $(<0.02)$. Furthermore the Behavior Counts Closed questions $(<0.01)$, Total questions $(<0.01)$, Simple reflections $(<0.01)$, Complex reflections $(<0.01)$, and Total reflections $(<0.01)$ were also discriminative.

\section{Nurse-specific fidelity}

In the post-hoc analysis, a considerable variation in nurses' MI-fidelity of the different MITI aspects within the intervention condition and control condition was found. 
Table 2: Reliability MITI-scoring for the two raters $(n=26)$

\begin{tabular}{|c|c|}
\hline Global Counselor Ratings MITI & KALPHA ordinal \\
\hline Direction & .63 \\
\hline Empathy & .55 \\
\hline Spirit & .62 \\
\hline Evocation & .58 \\
\hline Collaboration & .57 \\
\hline Autonomy/ Support & .17 \\
\hline Behavior Counts MITI & ICC \\
\hline \# Giving Information & $.45^{*}$ \\
\hline \% Open questions & $.89^{* *}$ \\
\hline \# Closed Questions & $.89^{* *}$ \\
\hline \# Open Questions & $.95^{* *}$ \\
\hline \# Total Questions & $.96^{* *}$ \\
\hline \% Complex reflections & .31 \\
\hline \# Simple Reflections & $.84^{* *}$ \\
\hline \# Complex Reflections & $.37^{*}$ \\
\hline \# Total Reflections & $.79^{* *}$ \\
\hline Reflections-to-questions ratio & $.94^{* *}$ \\
\hline \% MI-adherent responses & .24 \\
\hline \#MI-adherent responses & .12 \\
\hline \# MI-nonadherent responses & $.41^{* *}$ \\
\hline
\end{tabular}

${ }^{*} p<.05 ;{ }^{* *} p<.01 ; \mathrm{ICC}=$ Intraclass Correlation Coefficient, Single Measures; KALPHA= Krippendorff's alpha 
The nurses' individual behavior influenced the overall ratings of Ml-fidelity: One nurse of the control condition scored high on the Reflections-to- questions ratio explaining the higher -and threshold crossing- Reflections-to-questions ratio in the control condition (see table 2).

The fact that the nurses of the intervention condition used twice as many reflections as the nurses of the control condition four times as many Open Questions indicated that the intervention nurses used more $\mathrm{MI}$ required behaviors which means that $\mathrm{MI}$ took place in the intervention condition. This was hidden by just looking on the Summary Scores and belonging thresholds.

Within the intervention group, one of the two nurses of the intervention group scored higher and crossed the threshold proficiency for two additional Global Counselor Ratings, but did not cross another threshold of the other Summary Scores.

\section{Discussion and Conclusion}

The higher MITI mean ratings for treatment proficiency and treatment fidelity in four out of five Global Counselor Ratings, six out of nine Behavior Counts, threes out of five Summary Scores, and two out of two additional fidelity measures confirmed that Motivational Interviewing was applied in the Ml-based intervention condition of the underlying trial compared to its educational control condition.

MI-proficiency, referred to by crossing belonging thresholds, was not present for all available domains. Furthermore, on the basis of several available domains a statically significantly discrimination between both conditions could not be achieved. Thus, mean MI-proficiency scores were higher in the Ml-based intervention condition, but the levels are such that it can be debated whether the MI was delivered in such a way that it really influenced the patients' behavior. As such there is concern whether the Ml-based intervention is not advanced enough to make a change in the outcome of the pain rehabilitation treatment.

Sensitivity analysis by taking into account different ratings approaches (first rater, second rater, consensus approach of both raters together) seems to confirm the robustness of the findings, with the remark that these findings have to be seen in the light of rather mixed reliability. Findings of poor reliability were especially present in ratings, which did not exceed the threshold. For this reason, it cannot be concluded whether this is cause or consequence of the low reliability.

A general point of concern is that the current MITI-thresholds of proficiency are based upon expert opinion, and lack empirical support [25]. This implies also that it is currently unclear which level of $\mathrm{Ml}$ is minimally required to make a change [33].

Subsequently, as delivering $\mathrm{MI}$ is more complex than generally believed even in the case of regular and intensive training [34], an even higher level of competency than currently stated would be necessary to reach significant effects in outcome. 
An explanation for the mixed findings of inter-rater reliability could be that ratings of the first and second rater did not take place in the same time span, but three months later, which could have led to drift (decreased intra-rater variability and increased inter-rater reliability) [35]. On the other hand, the robustness of the Ml-fidelity findings during sensitivity analysis does not indicate this.

A comparison of the current findings with that of other intervention studies using $\mathrm{Ml}$ is hampered by the frequent use of other instruments than the MITI [36-38] or the usage of the previous version MITI 1.0 or 2.0 (e.g.[39]).

In the MITI, the more important fidelity measures are either related to change talk or sustain talk or to a behavior change at a later stage [20]. Therefore, important measures in the working chain of $\mathrm{Ml}$ are:

1. Empathy, and

2. Spirit [40].

Empathy, a fundamental factor in $\mathrm{MI}$, although non-specific in psychotherapy, was associated with better client outcomes in $\mathrm{Ml}$ delivered in the domain of addiction treatment [20]. Spirit, which is a combination of the global scores Autonomy/ Support, Evocation, and Collaboration, is a preliminary condition for change talk [20] Therein, the current finding of Evocation is embedded.

In a study of Forsberg et al. (2010), it was shown that some counselors with monthly training sessions needed two and a half year to reach the level of beginning proficiency for the global variable Spirit [35]. In two other studies assessing MI training effects, the thresholds for Spirit and Empathy were exceeded immediately after the training $[41,42]$.

In the study presented, the Reflection-to-questions ratio crossed the threshold for beginning proficiency, and seemed also in another study easy to learn and cross [35]. As a low amount of Complex reflections was found in the current study, it can be concluded that it was difficult for the nurses to provide Complex reflections. This was also mentioned in a study investigating $\mathrm{Ml}$ skills and counselor characteristics before, during, and after Ml-training [39] which is also in line with the statement that Complex reflections are one of the hardest to improve skills during training $[22,43,44]$.

The current finding that MI non-adherent responses were less common in the MI-based intervention condition compared to the educational control condition is as promising as it is known that MI non-adherent counselor behaviors hinders the subsequent occurrence of change talk which predicts behavior change $[13,18]$.

Another explanation for the fact that not all MITI-thresholds for beginning proficiency were crossed in the MI-based intervention condition could be due that the nurses had to follow a treatment-manual since they participated in a scientific study. The usage of a manual could have resulted in a situation in which the counselor felt less free to completely focus on actual needs of the client (e. g. pushing too hard for commitment in line with the manual) resulting in a negative impact on the achieving sufficient effect sizes $[4,45]$.

Whereas for most of the MITI variables higher mean ratings of MITI sub scores in the MI-based 
intervention condition compared to the control condition were found, it could not be statistically discriminate between the two conditions for most of the MITI variables. This finding is in accordance with the study of Maissi et al. (2011). It has to be said that some of the before mentioned studies did not use the MITI to discriminate between conditions at all, thus they were not included in this comparison $[15,43,44]$. Only one study indeed provided differences in MITI scores between $\mathrm{MI}$ conditions [41].

The current findings of differences in Ml-fidelity on nurse-level in the intervention condition in the post-hoc analysis are in line with a study on Ml-training effects, which also showed a broad variation in counselor functioning [46] and fluctuations over time [35].

In addition, it seems that some counselors are not able to acquire skills $[34,47]$ whereas others may easily learn the new technique, no matter the extent of training provided. This inter-person difference is in line with findings of this study and also with some other studies in the field of the effects of Ml-training [35,48,49]. As a consequence, a stricter selection policy (e.g. for empathy) before entry as a potential solution for undesired variations in counselors' Ml-functioning is advocated [47]. In addition, future study results should enlighten the most effective MI-training approaches as the important role of continuous supervision and feedback for Ml-practitioners is also reported elsewhere [39].

Although in a systematic review of O'Halloran et al. (2014) higher results for the effectiveness of Ml-intervention were yielded if fidelity assessment had taken place [12], Lundahl et al. (2013) reported in contrast in their systematic review that checking Ml-fidelity was inversely related to Ml-outcomes [5]. This was a surprising outcome, and those findings have to be seen in the light of two comments $[50,51]$ regarding methodological aspects of this review published. Apart from that, in accordance with Lundahl et al. [5] future studies are necessary and recommended to find an explanation for this phenomenon. The results of the underlying trial of this current study will be available next year and hopefully will shed some more light on this topic.

Although several studies investigating MI-fidelity do not even mention reliability between two or more raters $[44,52]$, results of the present and other studies (e.g. $[41,46])$ seem to underscore that a study of rater reliability is required and the procedure has to include regular rater meetings to prevent rater drift and probably prevent to hamper reliability [27].

At this moment, two overviews are available who describe a tool for treatment fidelity in health behavior change trials [53], and in trials using Motivational interviewing specifically [54].

Furthermore, several instruments are available for the assessment of MI quality. The MITI has shown to have good inter-rater-reliability [27] and predictive validity [28], and seems the most suitable if the specific focus is to specifically evaluate counselor behavior.

Furthermore, a limitation in the use of the MITI is that complex therapist (counselor) competence such as intentional or strategic use of MI may be insufficiently assessed [55] and one cannot evaluate the timing of interventions and techniques [56] as well as that the MITI does not take into account the context in which an interview takes place. A second limitation is the very time consuming coding of the sessions by means of the MITI; intensive training of the raters and continuous consulting between raters on intervention-specific topics and MITI-specific rating topics is necessary in order to rate unanimously. 
Some limitations of the present study need to be considered. First, the fidelity of the nurses in the Ml-intervention condition prior to or at the start of the trial was not assessed. This could have flawed the effectiveness of Ml-training and also the overall effectiveness of the Ml-intervention. Second, the first rater was not blinded for treatment allocation in the intervention versus control condition, which could have hampered validity of the findings. However, the finding that the blinded second rater confirmed the first rater's findings invalidates this supposition.

\section{Conclusion}

In general higher levels of MI-fidelity in the intervention condition were found as well as were it possible to partially discriminate between Ml-based intervention and education-based control condition. Although the quality of $\mathrm{MI}$ delivery as well as mixed inter-rater reliabilities of the fidelity scores leaves room for improvement, robust findings between the two raters and also their consensus approach were found.

Further analyses of the long-term effectiveness of the underlying trial will show whether a MIbased pretreatment is more effective compared to a pain education pre-treatment and whether this improves participation and functioning of patients with fibromyalgia and chronic musculoskeletal pain undergoing pain rehabilitation.

\section{Implications for Behavioral Health}

The present study confirms the need for rigor selection of Ml-counselors before training, and the important role of continuous supervision and feedback for Ml-practitioners in order to reach proper Ml-fidelity as well as the need for fidelity check in studies using MI. 


\section{Acknowledgements}

The research was funded by Adelante, the department of Rehabilitation Medicine, Maastricht University and in addition by a travel grant of the Dutch Arthritis Foundation (reumafonds). All authors worked independently of the funding body, and funding had no role in the collection, analysis, interpretation, writing or decision to submit this article.

Coding of VCM took place during a research visit at the MIC-lab of the Karolinska institutet, Stockholm, Sweden. The authors would also like to thank the whole team of the MIC lab. The considerable advice and coding as second rater of Judith Jelsma (JJ) is gratefully acknowledged. Draft versions of some results were presented at the International Conference on Motivational Interviewing in Amsterdam, the Netherlands, 18th, June 2014. 


\section{References}

[1] Miller WR, Rollnick S. Ten things that motivational interviewing is not. Behavioural and Cognitive Psychotherapy. 2009;37(2):129-140.

[2] Solomon DH, Iversen MD, Avorn J, et al. Osteoporosis telephonic intervention to improve medication regimen adherence: a large, pragmatic, randomized controlled trial. Archives of Internal Medicine. 2012;172(6):477-483.

[3] Burke B, Arkowitz H, Menchola M. The efficacy of motivational interviewing: a meta-analysis of controlled clinical trials. Journal of Consulting and Clinical Psychology. 2003; $71(5): 843-861$.

[4] Hettema J, Steele J, Miller WR. Motivational interviewing. Annual Review of Clinical Psychology. 2005;1:91-111.

[5] Lundahl B, Moleni T, Burke BL, et al. Motivational interviewing in medical care settings: a systematic review and meta-analysis of randomized controlled trials. Patient Education and Counseling. 2013;93(2):157-168.

[6] Rubak S, Sandbaek A, Lauritzen T, Christensen B. Motivational interviewing: a systematic review and meta-analysis. Br J Gen Pract. 2005;55(513):305-312.

[7] Lundahl B, Burke BL. The effectiveness and applicability of motivational interviewing: A practice-friendly review of four meta-analyses. Journal of Clinical Psychology. 2009;65(11):1232-1245.

[8] Ang DC, Kaleth AS, Bigatti S, et al. Research to Encourage Exercise for Fibromyalgia (REEF): Use of motivational interviewing design and method. Contemporary Clinical Trials. $2011 ; 32(1): 59-68$.

[9] Habib S, Morrissey S, Helmes E. Preparing for pain management: a pilot study to enhance engagement. Journal of Pain. 2005;6(1):48-54. 
[10] Leonhardt C, Keller S, Chenot JF, et al. TTM-based motivational counselling does not increase physical activity of low back pain patients in a primary care setting-A cluster-randomized controlled trial. Patient Education and Counseling. 2008;70(1):50-60.

[11] Vong SK, Cheing GL, Chan F, So EM, Chan CC. Motivational enhancement therapy in addition to physical therapy improves motivational factors and treatment outcomes in people with low back pain: a randomized controlled trial. Archives of Physical Medicine and Rehabilitation. 2011;92(2):176-183.

[12] O'Halloran PD, Blackstock F, Shields N, et al. Motivational interviewing to increase physical activity in people with chronic health conditions: a systematic review and meta-analysis. Clinical Rehabilitation. 2014;28(12):1159-71.

[13] Miller WR, Rose GS. Toward a theory of motivational interviewing. American Psychologist 2009;64(6):527-537.

[14] Rollnick S, Butler CC, Kinnersley P, Gregory J, Mash B. Motivational interviewing. British Medical Journal. 2010;340:c1900.

[15] Gaume J, Bertholet N, Faouzi M, Gmel G, Daeppen J-B. Counselor motivational interviewing skills and young adult change talk articulation during brief motivational interventions. Journal of Substance Abuse Treatment. 2010;39(3):272-281.

[16] Bellg AJ, Borrelli B, Resnick B, et al. Enhancing treatment fidelity in health behavior change studies: best practices and recommendations from the NIH Behavior Change Consortium. Health Psychology. 2004;23(5):443-451.

[17] Perepletchikova F, Treat TA, Kazdin AE. Treatment integrity in psychotherapy research: analysis of the studies and examination of the associated factors. Journal of Consulting and Clinical Psychology. 2007;75(6):829-841.

[18] Moyers TB, Martin T. Therapist influence on client language during motivational interviewing sessions. Journal of Substance Abuse Treatment. 2006;30(3):245-251.

[19] Lovejoy TI, Heckman TG, Suhr JA, Anderson T, Heckman BD, France CR. Telephone-administered motivational interviewing reduces risky sexual behavior in HIV-positive late middle-age and older adults: a pilot randomized controlled trial.

AIDS Behav. 2011;15(8):1623-1634. 
[20] Apodaca TR, Longabaugh R. Mechanisms of change in motivational interviewing: a review and preliminary evaluation of the evidence. Addiction. 2009;104(5):705-715.

[21] Miller W, Mount K. A small study of training in motivational interviewing: does one workshop change clinician and client behavior? Behav Cogn Psychother. 2001;29:457-471.

[22] Miller W, Yahne C, Moyers T, Martinez J, Pirritano M. A randomized trial of methods to help clinicians learn motivational interviewing. J Consult Clin Psychol. 2004;72(6):1050-1062.

[23] Moyers TB, Martin T, Manuel JK, Hendrickson SM, Miller WR. Assessing competence in the use of motivational interviewing. Journal of Substance Abuse Treatment. 2005;28(1):19-26.

[24] Mertens VC, Goossens ME, Verbunt JA, Koke AJ, Smeets RJ. Effects of nurse-led motivational interviewing of patients with chronic musculoskeletal pain in preparation of rehabilitation treatment (PREPARE) on societal participation, attendance level, and cost-effectiveness: study protocol for a randomized controlled trial. Trials. 2013;14(1):90.

[25] Moyers TB, Martin T, Manuel JK, Miller WR, Ernst D. Revised Global Scales: Motivational Interviewing Treatment Integrity 3.1.1 (MITI 3.1.1). 2010; http://casaa.unm.edu/download/MITI3_1.pdf.

[26] Pierson HM, Hayes SC, Gifford EV, et al. An examination of the Motivational Interviewing Treatment Integrity code. Journal of Substance Abuse Treatment. 2007;32(1):11-17.

[27] Forsberg L, Kallmen H, Hermansson U, Berman AH, Helgason AR. Coding counsellor behaviour in motivational interviewing sessions: inter-rater reliability for the Swedish Motivational Interviewing Treatment Integrity Code (MITI). Cognitive Behaviour Therapy. 2007;36(3):162-169.

[28] Forsberg L, Berman AH, Kallmen $H_{1}$ Hermansson U, Helgason AR. A test of the validity of the Motivational Interviewing Treatment Integrity Code. Cognitive Behaviour Therapy. 2008;37(3):1-9.

[29] Moyers TB, Miller WR. Is low therapist empathy toxic? Journal of Addictive Behaviors. 2013;27(3):878-884.

[30] Cicchetti DV. Guidelines, criteria, and rules of thumb for evaluating normed and standardized assessment instruments in psychology. Psychological Assessment. 1994;6(4):284-290.

[31] Krippendorff K. Agreement and Information in the Reliability of Coding. Communication Methods and Measures. 2011;5(2):93-112. 
[32] Hayes AF, Krippendorff K. Answering the Call for a Standard Reliability Measure for Coding Data. Communication Methods and Measures. 2007;1(1):77-89.

[33] Miller WR, Rollnick S. The effectiveness and ineffectiveness of complex behavioral interventions: Impact of treatment fidelity. Contemporary Clinical Trials. 2014;37(2):234-241.

[34] Bohman B, Forsberg L, Ghaderi A, Rasmussen F. An Evaluation of Training in Motivational Interviewing for Nurses in Child Health Services. Behavioural and Cognitive Psychotherapy. 2013;41(03):329-343.

[35] Forsberg $L$, Forsberg LG, Lindqvist $H$, Helgason AR. Clinician acquisition and retention of Motivational Interviewing skills: a two-and-a-half-year exploratory study.

Substance Abuse Treatment Prevention and Policy. 2010;5:8.

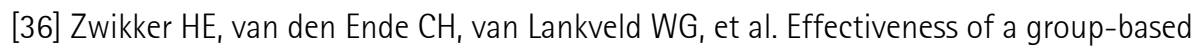
intervention to change medication beliefs and improve medication adherence in patients with rheumatoid arthritis: A randomized controlled trial. Patient Education and Counseling. 2014;94(3):356-361.

[37] Noordman J, de Vet E, van der Weijden T, van Dulmen S. Motivational interviewing within the different stages of change: An analysis of practice nurse-patient consultations aimed at promoting a healthier lifestyle. Social Science \& Medicine. 2013;87(0):60-67.

[38] Jansink R, Braspenning J, Van Der W, Niessen L, Elwyn G, Grol R. Nurse-led motivational interviewing to change the lifestyle of patients with type 2 diabetes (MILD-project): protocol for a cluster, randomized, controlled trial on implementing lifestyle recommendations. BMC Health Service Research. 2009;9:19.

[39] Carpenter KM, Cheng WY, Smith JL, et al. „Old dogs" and new skills: how clinician characteristics relate to motivational interviewing skills before, during, and after training. Journal of Consultant and Clinical Psychology. 2012;80(4):560-573.

[40] Miller WR, Rollnick S. Motivational Interviewing: Preparing People for Change. (Second Ed.) ed. New York: The Guilford Press; 2002.

[41] Daeppen JB, Fortini C, Bertholet N, et al. Training medical students to conduct motivational interviewing: a randomized controlled trial. Patient Education and Counseling. 2012;87(3):313-318. 
[42] Maissi E, Ridge K, Treasure J, et al. Nurse-led psychological interventions to improve diabetes control: assessing competencies. Patient Education and Counseling. 2011;84(2):e37-43.

[43] Baer JS, Rosengren DB, Dunn CW, Wells EA, Ogle RL, Hartzler B. An evaluation of workshop training in motivational interviewing for addiction and mental health clinicians. Drug and Alcohol Dependence. 2004;73(1):99-106.

[44] Van Eijk-Hustings YJL, Daemen L, Schaper NC, Vrijhoef HJM. Implementation of Motivational Interviewing in a diabetes care management initiative in the Netherlands. Patient Education and Counseling. 2011;84(1):10-15.

[45] Lundahl BW, Kunz C, Brownell C, Tollefson D, Burke BL. A meta-analysis of motivational interviewing: Twenty-five years of empirical studies. Research on Social Work Practice. 2010;20(2):137-160.

[46] Maissi E, Ridge K, Treasure J, et al. Nurse-led psychological interventions to improve diabetes control: assessing competencies. Patient Education and Counseling. 2011;84(2):37-43.

[47] Lindhardt CL, Rubak S, Mogensen O, Hansen HP, Lamont RF, Jorgensen JS. Training in motivational interviewing in obstetrics: a quantitative analytical tool. Acta Obstetricia et Gynecologica Scandinavica. 2014;93(7):698-704.

[48] Miller WR, Moyers TB, Arciniega L, Ernst D, Forcehimes A. Training, supervision and quality monitoring of the COMBINE Study behavioral interventions. Journal of Studies on Alcohol and Drugs Supplement. 2005(15):188-195; discussion 168-189.

[49] Moyers TB, Manuel JK, Wilson PG, Hendrickson SML, Talcott W, Durand P. A Randomized Trial Investigating Training in Motivational Interviewing for Behavioral Health Providers. Behavioural and Cognitive Psychotherapy. 2008;36(02):149-162.

[50] Mullin D. Seeking clarification and corrections of motivational interviewing meta-analysis. Patient Education and Counseling. 2014;95(2):295.

[51] Van der Wouden JC, Rietmeijer C. Motivational interviewing in the medical care setting. Patient Education and Counseling. 2014;96(1):142.

[52] Robbins LB, Pfeiffer KA, Maier KS, Ladrig SM, Berg Smith SM. Treatment fidelity of motivational interviewing delivered by a school nurse to increase girls' physical activity. The Journal of School Nursing. 2012;28(1):70-78. 
[53] Borrelli B. The Assessment, Monitoring, and Enhancement of Treatment Fidelity In Public Health Clinical Trials. Journal of Public Health Dentistry. 2011;71(s1):S52-S63.

[54] Jelsma JG, Mertens VC, Forsberg L, Forsberg L. How to Measure Motivational Interviewing Fidelity in Randomized Controlled Trials: Practical Recommendations. Contemporary Clinical Trials. 2015;43:93-99.

[55] Madson MB, Campbell TC. Measures of fidelity in motivational enhancement: a systematic review. Journal of Substance Abuse Treatment. 2006;31(1):67-73.

[56] de Jonge JM, Schippers GM, Schaap CPDR. The Motivational Interviewing Skill Code: Reliability and a Critical Appraisal. Behavioural and Cognitive Psychotherapy. 2005;33(03):285-298. 


\section{Chapter 4}

How to Measure Motivational Interviewing Fidelity in Randomized Controlled Trials:

Practical Recommendations.

Jelsma J.G, Mertens V.-C. (shared 1st authorship) Forsberg L. Forsberg L.

Contemporary Clinical Trials. 2015 July; 43:93-99. DOI: 10.1016/j.cct.2015.05.001.

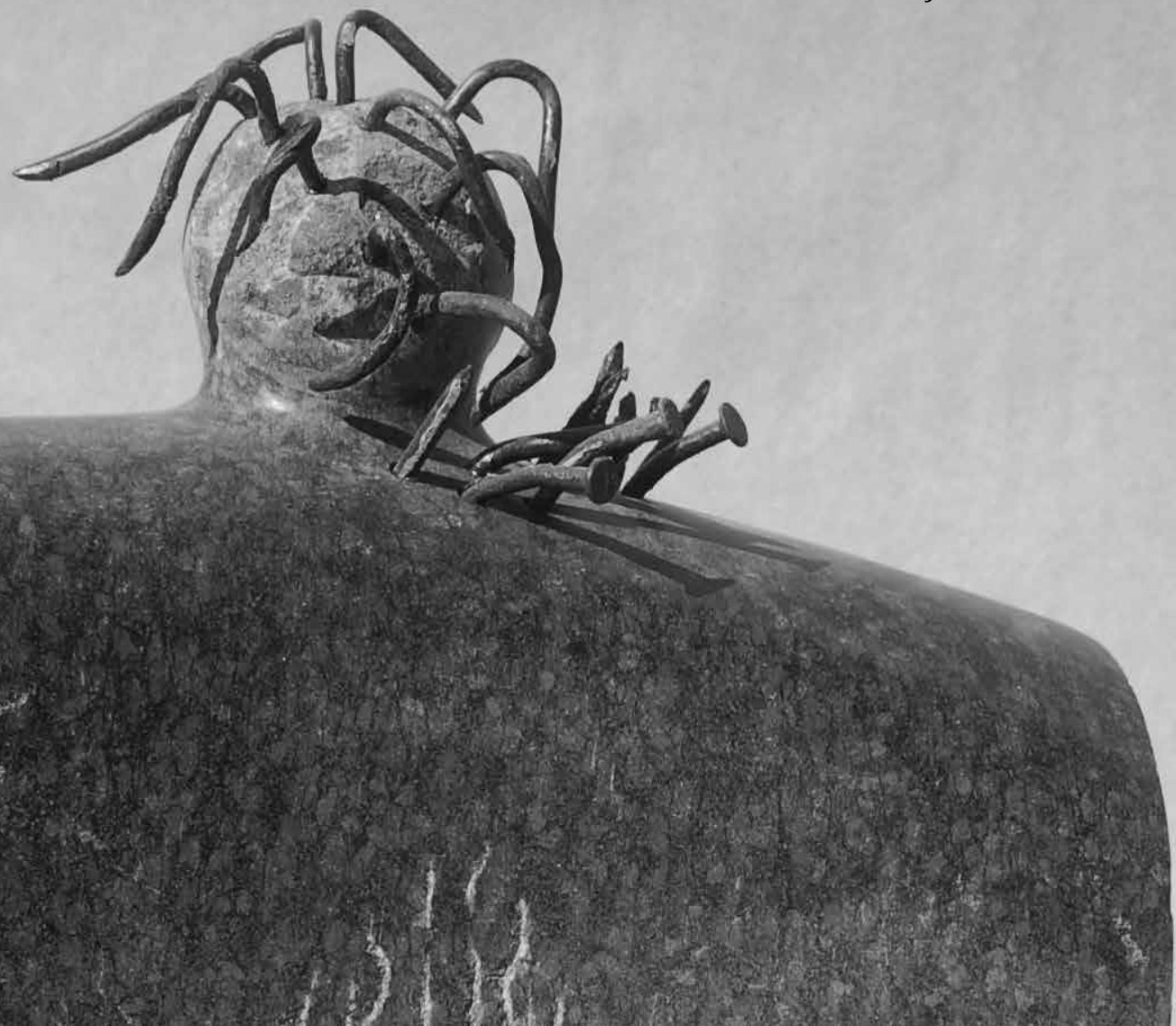




\section{Abstract}

Many randomized controlled trials in which Motivational Interviewing (MI) is a key intervention make no provision for the assessment of treatment fidelity. This methodological shortcoming makes it impossible to distinguish between high- and low-quality MI interventions, and, consequently, to know whether $\mathrm{Ml}$ provision has contributed to any intervention effects. This article makes some practical recommendations for the collection, selection, coding and reporting of MI fidelity data, as measured using the Motivational Interviewing Treatment Integrity code. We hope that researchers will consider these recommendations and include MI fidelity measures in future studies.

\section{Introduction}

"Motivational Interviewing (MI) is a collaborative, goal-oriented style of communication with particular attention to the language of change. It is designed to strengthen personal motivation for and commitment to a specific goal by eliciting and exploring the person's own reasons for change within an atmosphere of acceptance and compassion"[1]. MI has been shown to be superior to no intervention, and as efficacious as other evidence-based interventions in systematic reviews and meta-analyses across a variety of different problem behaviors and health care settings [2-6].

A randomized controlled trial (RCT) tests whether an intervention is efficacious in an ideal situation by maximizing internal validity through controlling all variables except the intervention to be tested. A controlled clinical trial tests instead whether an intervention is effective in a real life setting, maximizing the external validity to ensure generalizability [7]. In order for the move from an efficacy trial to an effectiveness trial to be successful, it is important to have identified the active mechanism of the intervention tested in the efficacy study [8]. In respect of behavior change intervention research, the reporting of treatment fidelity is likely to improve the credibility of evidence that results from a trial [9]. Treatment fidelity refers to the "methodological strategies used to monitor and enhance the reliability and validity of behavioral interventions" [9] (p.443). Treatment fidelity in MI has predictive validity in respect of patient behavior following the intervention [10-12]. However, many research trials conducted have failed to assess treatment 
fidelity of the intervention that is being delivered. This makes it impossible to ascertain whether the result can accurately be attributed to the $\mathrm{MI}$ intervention, that is, whether we can in fact be sure that $\mathrm{Ml}$ is the actual working mechanism that is "doing the job" [13]. Miller and Rollnick (2014) suggest that treatment fidelity should be assessed throughout a study, through a reliable assessment procedure ('coding'), and be reported in a manner that allows for comparison across trials [13].

The aim of this paper is to provide guidance to researchers in respect of assessing and reporting $\mathrm{Ml}$ treatment fidelity. The practical recommendations offered are important to consider in designing, developing and conducting research, including in grant applications.

\section{The Motivational Interviewing Treatment Integrity (MITI) Code}

The Motivational Interviewing Treatment Integrity (MITI) code is the most frequently used [13] instrument for assessing MI fidelity in RCTs [14-16]. The MITI has been derived from the Motivational Interviewing Skill Code (MISC) [17], and while reducing the MISC's complexity and length [18], the MITI focuses exclusively on the verbal behavior of the practitioner, and does not take client responses into account [15]. The MITI is continuously revised and improved. Almost ten years ago, MITI version 2.0 was being used, and at present, the MITI 4.1 has just been published. Definitions of variables that measure important aspects of $\mathrm{MI}$ practice are improved in each new version, with the aim to carefully follow and progress developments in MI research. Every previous version of the MITI instrument [14] has been shown to be reliable $[15,16,19,20]$ and valid $[14,16]$. The recently published MITI 4.1 has been shown to have face validity, but the collection of data regarding its validity and reliability is still underway.

In the recent MITI 4.1, substantial changes have been made in comparison to MITI 3.1.1. The authors claim that the two versions are not comparable, and advise researchers to use the MITI 4.1 from now on. However, before MITI 4.1 may be used more widely, the instrument has to show predictive validity at least in respect of some problem behaviors and in some languages, and coders using the new instrument need to be able to achieve an adequate inter-rater reliability. In order to assist researchers both in conducting treatment fidelity assessment in future research, and in interpreting research conducted so far, both versions of the MITI are briefly discussed in this paper.

The MITI comprises two separate components: global variables and behavior counts. In both versions of the MITI, a 20-minute segment is used both for the behavior counts and for rating the global variables. The global ratings reflect the coder's overall impression of how well or poorly 
a practitioner performed in a certain aspect of $\mathrm{MI}$ practice, rated on a five point Likert scale. In MITI 3.1.1, the global scales are Empathy, Evocation, Collaboration, Autonomy/Support, and Direction. In both MITI 3.1.1 and MITI 4.1, the Empathy rating captures how well the practitioner understands the client's perspective, experiences, and feelings. In MITI 3.1.1, the global variable MI Spirit combines the ratings of Evocation, Collaboration and Autonomy/Support (by taking an average of the ratings of all three variables). In MITI 4.1, the MI Spirit variable is replaced by the variables Cultivating Change Talk, Softening Sustain Talk, and Partnership. This modification emphasizes the importance of the practitioner adapting her behavior in response to client utterances. A further change in respect of the global variables in MITI 4.1 is that the Direction variable has been removed.

The behavior counts are intended to capture specific practitioner verbal behaviors that are relevant to good practice of MI. The MITI 4.1 retains the behavior count categories Giving Information, Simple Reflections, and Complex Reflections. However, some other behavior count categories have been changed in the MITI 4.1. First, the two categories Open and Closed Questions have been combined into one single Questions category. Second, the category for MI Adherent practitioner behavior has been divided into several categories for subtypes of such behavior, each of which is given a separate code: Seeking Collaboration, Emphasizing Autonomy, and Affirm. Third, the category for MI Non-Adherent practitioner behavior in MITI 3.1.1 has been split up into Confront, and Persuade (with and without permission) in MITI 4.1. For a more detailed discussion of the different variables in the two different versions of the MITI we refer to the MITI 3.1.1 manual [14] and the MITI 4.1 manual [21].

\section{Assessing Treatment Fidelity in MI Sessions}

Prior to the start of an RCT where MI is one of the interventions being tested, it is important to consider the following three things: 1) which samples of MI practice (sessions) will be collected and selected for fidelity assessment; 2) who will do the assessment (coding) of these sessions; and 3) how will the results be reported. These three essential questions will be discussed in detail below.

\subsection{Collection of Audio-Recorded Sessions}

Since audio-recorded sessions are used to assess treatment fidelity -that is, what really happened in the interaction between practitioner and client- it is important to audio record all, or as much as possible, of the conducted conversations. Recording all sessions allows the researcher to minimize selection bias, which is easily introduced if practitioners are permitted to select the sessions submitted for treatment integrity assessment themselves [22]. Approval by the relevant ethics review board, and the consent of clients and practitioners, have to be obtained prior to the audio 
recording. Informing clients and practitioners that the data will be anonymized might make them less reluctant to consent. In addition, providing practitioners with digital audio recording devices (and checking compliance throughout the study) could assist in obtaining the full spectrum of conversations.

\subsection{Selection of Samples for Assessment}

A random representative sample of the collected audio-recorded sessions should be selected. It will often not be possible to assess the treatment fidelity of all sessions, but coding multiple work samples from each practitioner provides a more accurate assessment of his or her proficiency [23]. So the question is, how large should this representative sample be, keeping in mind that studies have different design in respect of the number of participating practitioners, the number of sessions per client, and so forth.

In previously conducted RCTs where attempts have been made to assess treatment fidelity, between 11-32\% of the total number of sessions were assessed (e.g. [24] (25\%); [25] (16\%); [26] (11\%); [27] (28\%); [28] (25\%); [29] (23\%); [30] (32\%)). However, the study of Smith et al. (2012) is an exception to this since $100 \%$ of recorded sessions were assessed, although the total number of sessions in this study only comprised 38 [31]. In studies where the intervention was delivered by more than one practitioner, 10-17 sessions per practitioner were selected for assessment ([32], $(n=17) ;[26](n=10))$ to represent a reliable overview of the quality of the individual practitioner throughout the study period. El-Mallakh et al. (2012) assessed 18 sessions (25\% of total sessions) [28], and McCarthy et al. (2014) assessed 4 sessions (20\% of total sessions) [33] throughout the study period (both with only one practitioner delivering the intervention), providing an indication of the MI skill fluctuation in the practitioner delivering the intervention over time.

Some studies require a comparison of overall group results (average of multiple practitioners), for example, when usual care conditions containing an attention control intervention without an $\mathrm{MI}$ component and an $\mathrm{Ml}$ intervention condition are compared (e.g. [31]), or when practitioners with different backgrounds/experience are compared (e.g. [29] $(n=19)$ ). In the study of Smith et al. (2012), one practitioner delivered both the intervention and the control arm of the study [31]. Here, it was examined if $\mathrm{MI}$ was more pronounced in the intervention group than in the control group by assessing 20 intervention sessions and 18 control sessions.

Audio-recorded session may vary in length between 10 min and over an hour. The MITI is used to assess a 20-minute segment of each session. It may be the case that sessions shorter in length than 20 min could not be reliably coded using the MITI [20]. For longer sessions, it may be hard to decide how to choose the segment submitted for assessment, in particular since the quality of a practitioner's MI practice might fluctuate throughout a session. This was, for example, found in the psycholinguistic study of Amrhein et al. (2003), in which there was an explicit requirement for practitioners to agree a change plan with the client at the end of a face-to-face-session. In cases where the client was not yet ready to agree to a change plan, this led practitioners to adopt a directive (rather than a collaborative) approach with the client towards the end of their sessions, when the change plan had to be discussed. This resulted in low MI fidelity, despite the beginning of the sessions being at an adequate level of MI fidelity [34]. 
Different approaches may be adopted to decide which segment of a session should be coded. These range from decisions being made based on the content of sessions, where the coding of segments that are off-topic or which do not focus on the target behavior is avoided, to the selection at random of segments to be coded. To improve generalizability, the random sample should comprise some segments from the beginning of sessions, some from the middle, and some from the end of sessions, so that the whole spectrum of sessions' content is captured. However, it may be advisable to avoid coding the very beginning and the very end of audio-recordings, since these will often contain off topic material and talk not related to the targeted behavior change (e.g. information about the trial or scheduling of new appointments), and might therefore introduce bias.

Most RCTs run over a year or more, due to the recruitment phase and subsequent follow-up. Considering that practitioners will experience changes during this time, and that their level of MI skill may fluctuate [35], it is important to select sessions from different points in time throughout the entire intervention period, to get a complete and representative picture of practitioners' MI skill. In particular, if practitioners receive ongoing supervision or additional training, improvements in MI proficiency could be expected $[25,36]$. Moreover, the client also influences the session; therefore it is desirable to include sessions with different clients, who are at different stages of change, in the sample. For example, Noordman et al. (2013) found that nurses applied more MI skills when their patients were in the so-called preparation phase of behavior change [37].

In most research trials more than one intervention session is delivered to participants. To get an accurate picture of the quality of the delivered $\mathrm{MI}$ across the whole intervention period, not only the first session with a participant should be selected, but subsequent ones as well.

\section{Coding}

\subsection{Coders}

Several questions will need to be considered before it is possible to assess MI fidelity. The first question is: Who will code the sessions? To the best of our knowledge, there are only a few permanent coding labs consisting of a group of coders and with established procedures for maintaining inter-rater reliability. These have been founded for the purposes of assessing treatment fidelity in research, for carrying out quality assurance of routine clinical practice, and for providing feedback to participants as part of MI trainings. These coding labs are MIC Lab (Karolinska Institutet, Stockholm, Sweden, director: Lars Forsberg), KoRus (Bergen, Norway, directors: Nina-Elin Andresen and Solveig Storbakken), and the research group that developed the MITI and MISC instruments, based at CASAA, University of New Mexico (Albuquerque, United States, leader: Terri Moyers). To involve one of these in one's research project may be the best option, since these coding labs strive to maintain a gold standard for inter-rater reliability. 
However, there may be several reasons (including language related ones) that it may not always be feasible to involve an established coding lab in one's research projects. As an alternative in cases where this may not be possible, individuals associated with the MI Network of Trainers (the MINT network) in many countries code sessions as part of their MI trainings, and who might more easily be able to learn to code reliably. There are also at any given time likely to exist several non-permanent coding labs, established for the purposes of assessing treatment fidelity in particular on-going research projects, but which tend to be dissolved once these projects come to an end.

Earlier research has shown that training inexperienced individuals (e.g. students) to use the MITI is possible $[25,27,29,31,38]$. However, training people to use the MITI is time consuming (more than $40 \mathrm{~h}$ ). It is necessary to create an environment where (prospective) coders can discuss questions and uncertainties and reach a consensus when faced with difficulties during the coding process. To minimize the risk of drift among coders and to promote adherence to the MITI manual, it is advised to assess coders' competence and calculate their inter-rater reliability prior to permitting coding of the study sample to begin, and to only allow coders to begin to code the study material once they have reached an adequate inter-reliability (see below for recommendations regarding what an adequate inter-rater reliability is).

\subsection{Inter-rater Reliability}

Assessing and reporting coders' inter-rater reliability is absolutely crucial, but something that has often been neglected in MI research [13]. In order for reported results to be reliable, it is advised to have (at least) one second coder. At present, there are no official recommendations regarding the proportion of sessions that should be coded by two coders (double-coded) for the purposes of assessing inter-rater reliability. Most previously conducted RCTs report that 4\%-32\% of sessions were double-coded (e.g. [38] (32\%; $n=11) ;$ [32](4\%; $n=6) ;$ [39] (20\%; $n=19) ;$ [27] (8\%; $n=115)$; [25](30\%; $n=54) ;$ [40](10\%; n unknown); [22](25\%; n unknown) [36] (27\%: $n=15))$. However, in some studies, all sessions were coded by at least two of the coders [28, 31, 41, 42]. Having a large proportion, or indeed all sessions, double-coded will of course increase the validity of the results, but will not always be feasible were the total number of audio-recorded sessions is large.

It is important that coders rate the exact same segment of each of the recorded sessions when inter-rater reliability is tested. This requires that the exact start and end time of each segment, along with the first and the last sentence of the segment, is noted and made available to coders prior to their coding. It is also helpful to make a note of utterances that could be interpreted differently (e.g. the subtypes of MI Adherent, the subtypes of MI Non-Adherent, and Complex Reflections), along with the time at which they occurred, since this will facilitate subsequent discussion among coders. It is also helpful for coders to make a note of observations that are relevant to the global ratings, such as things that affected their rating of a particular global variable either positively or negatively. This makes it easier for coders to provide concrete examples to justify their rating.

The MITI 4.1 manual [21] (as previous iterations of the MITI) advises that only audio-recordings are to be used for the purposes of the coding process. It is fine to use transcripts of sessions 
for the purposes of training coders (and indeed, it is probably necessary to do so at least in the beginning of coder training, since coders need to be able to discuss in detail nuances in each utterance in order to develop an understanding of how the variables are distinguished, etc.), but the use of (only) transcripts for the purposes of assessing treatment fidelity in sessions is clearly against the recommendations. Using audio-recordings only, rather than transcripts, saves much time (no time needed for transcription). More importantly, however, audio-recordings allow for vital aspects such as voice intonation, which are lost when sessions are transcribed, to be taken into account. This is crucial in respect of, for example, discriminating between a Reflection and a Question, or when deciding whether an utterance should receive a Persuade or Confront (MITI 4.1) or an MI Non-Adherent (MITI 3.1.1.) code. A quiet environment without distraction is all that is needed for the coding of audio-recorded sessions. The only disadvantage of audio-recordings could be the unblinding of the coder to the identities of the practitioner or the client, if these are persons familiar to the coder.

\subsection{Study Specific Situations}

In research trials, the $\mathrm{MI}$ intervention is sometimes accompanied by other study specific requirements (e.g. if weight is a study outcome, measuring weight progression at each session might be required), or study specific manuals that need to be followed or used in the interaction. Although such requirements are intended to assist in promoting behavior change, they might impact on the quality of practitioners' MI practice [5]. If certain study-specific situations are not covered by the existing MITI manual, coders should determine how to code these deviations prior to beginning to code the study material [14]. There may also be other aspects that might not be covered by the MITI. For example, client encounters that serve as attention-control interventions may be difficult to rate in respect of the Direction and Evocation variables (in MITI 3.1.1), if they are non-directive and/or do not relate to a specified target behavior (both these variables require a target behavior [14]). For such encounters, it may be preferable to omit them. In MITI 4.1, this problem may arise in respect of the variables Cultivating Change Talk and Softening Sustain Talk, which both require that the coder is aware of the designated target behavior in the interaction.

\section{Reporting}

\subsection{Reporting MITI Results}

MITI results are reported in a variety of ways in the literature. Some studies report results in respect of all MITI variables, while others present results according to the standard way of reporting MITI results, by providing outcomes for the global variables and the behavior count summary scores. For materials coded using MITI 3.1.1, the standard approach would report the ratio of Complex Reflections to Simple and Complex Reflections, the ratio of Open Questions to Open 
and Closed Questions, the ratio of MI Adherent to MI Adherent and MI Non-Adherent utterances, and the ratio of Reflections to Questions, along with the scores for the global variables Empathy, MI Spirit and Direction. In respect of these behavior count summary scores and global variables, there are recommended thresholds for Beginning Proficiency and Competency, which are based on expert opinion and in need of validation [14]. In the MITI 4.1 this approach would report the global components Technical Global (Cultivating Change Talk and Softening Sustain Talk), Relational Global (Partnership and Empathy), and the summary scores percentage of Complex Reflections (of all Reflections), the ratio of Reflections to Questions, Total MI adherent (Seeking Collaboration, Affirm, and Emphasizing Autonomy) and Total MI Non-Adherent (Confront and Persuade) [21]. In MITI 4.1, the recommended thresholds are for Fair and Good MI practice.

Some studies report the average MITI results for different interventions [25, 29, 31, 36], others for different (groups of) practitioners [39, 43], and some for individual practitioners over time $[28,43]$. In one study, the MITI results of two practitioners were weighed based on the number of participants each practitioner had counseled to obtain an overall MI fidelity for the study intervention [26]. In the study of Ang et al. (2013), the results for individual MITI variables were not reported, but the proportion of audio-recorded sessions that reached the recommended threshold for Beginning Proficiency (in all MITI variables) was reported for both the MI and the control condition [24]. These differences in the reporting MITI results depend in part on the research question asked in various studies (whether the researchers are interested in improvements in Ml skill over time, or in the respective efficacy or effectiveness of different interventions, or in differences in $\mathrm{Ml}$ skill across practitioners, etc.).

In MITI 4.1, the authors encourage the full reporting of all MITI scores in clinical trials where MITI is used to assess treatment fidelity, since this data, when related to clinical outcomes, could provide empirical support needed to confidently establish recommended thresholds for Fair and Good MI practice, in particular with regard to the MI Adherent and MI Non-Adherent variables, where data is currently lacking and in respect of which no thresholds have been recommended [21].

\subsection{Reporting inter-rater reliability}

A wish for the future is that inter-rater reliability scores are presented in all scientific articles. It is striking and puzzling that this is not done routinely at the moment. Reaching a sufficient degree of inter-rater reliability is difficult and requires intensive training, collaboration and ongoing discussion, which might be a reason that these scores are not reported frequently. In the literature to date, the inter-rater reliability reported for different MITI variables has varied tremendously, ranging from 'poor' to 'excellent' (see below for thresholds) (e.g. $[25,36])$.

It is important to report the inter-rater reliability in each of the variables [44]. So for example, both the inter-rater reliability of the Complex Reflections behavior count and the Simple Reflections behavior count should be reported, and rather than just the inter-rater reliability of the coding of the behavior count summary score for the ratio of Complex Reflection to Simple and Complex Reflections. This is because measurement errors could be overestimated in the summary scores. In MITI 3.1.1, this was a problem, in particular in respect of some variables that did not occur 
frequently, most notably the MI Adherent and MI Non-Adherent variables. For example, when the summary score for the ratio of MI Adherent to MI Adherent and MI Non-Adherent behavior is calculated (dividing the total of MI Adherent utterances by the total of MI Adherent and MI Non-Adherent utterances), it could be the case that one coder did not detect any MI Adherent utterances, and therefore has summary score of $0 \%$, while another coder who has detected at least one MI Adherent utterances might get a score of 100\%. In this case, the inter-rater reliability score would be low for the summary score, even though it would have been acceptable for the raw variables. In MITI 4.1, this problem may have been evaded in respect of these variables, since the MI Adherent and MI Non-Adherent scores are a summation instead of a percentage.

Judging from the existing literature, it may be easier to obtain sufficient inter-rater-reliability in respect of some MITI variables than others. For example, it seems that coders more easily code the global Direction variable and behavior counts Giving Information and Reflections (e.g. [27, 36] reliably. The global variable Autonomy/Support and the MI Adherent and MI Non-Adherent behavior counts seem considerably more difficult for coders to reach agreement on. In respect of these, along with the Reflections to Questions ratio, the reported inter-rater reliability has ranged 'poor' to 'fair' in several studies (e.g. [27, 36]).

It is important to note that some MITI variables may be more important with regard to predicting outcome, and therefore also more important to be able to code reliably. For example, high Empathy scores and low levels of MI Non-Adherent behavior may be predictive of successful client outcome $[12,45]$. Reporting the inter-rater reliability for those variables is therefore vital.

If the inter-rater reliability between coders is high, it is fine to report the results of the first coder only. If the inter-rater reliability is medium or low, one might consider using a more pragmatic approach, such as presenting the results of each coder separately, or presenting an aggregated score of both coders' ratings. Coders could decide to discuss their respective ratings and reach consensus in respect of the global scores for each of the variables, and to use arithmetic averaging for the behavior counts. This method offers a pragmatic and practical solution based upon two coders that provides an indication of the level of MI fidelity.

\subsection{Calculation of Inter-rater reliability}

The most common way to calculate inter-rater reliability scores for the MITI global variables and behavior counts is by intra-class correlation coefficients (ICC), using a two-way mixed model with absolute agreement [46]. ICC scores are generally compared against the following benchmark values [47]: $0.40=$ poor; $0.40-0.59=$ fair; $0.60-0.74=$ good, and $0.75-1.00=$ excellent. Hayes and Krippendorff (2007) argue that the calculation of inter-rater reliability scores for ordinal variables (the global MITI variables) should not be done using Cronbach's alpha or percent agreement [48]. Cronbach's alpha is a statistic for interval-level data, which is not sensitive to the level of agreement in judgment, but only acts as quantification method of judgment [48]. Percent agreement is limited to nominal or categorical levels, can only be calculated for two coders, and there is no correction for the minimal chance of agreement of scoring the same variable [49]. Furthermore, it is proposed that Krippendorff's Alpha (KALPHA) [49] is used instead of ICCs for calculating the (ordinal) global MITI variables. This is more suitable where data might be missing [44]. 
Additionally, a restricted range, which the global MITI variables have, reduces the utility of the ICC for assessing inter-rater reliability [32]. At the moment, KALPHA is frequently used in content analysis, although not in the context of treatment fidelity assessment. The KALPHA can be used regardless of the number of coders and levels of measurement, and can deal with missing scores [48]. KALPHA takes into account the prevalence of answer categories (and not the amount of existing categories), meaning that the rarity of categories will impact KALPHA. As norm for good reliability testing a KALPHA of 0.80 has been suggested [50].

It is worth noting that variables that are generally scored across a smaller range of the intended scale (e.g. the Direction global scale in the MITI 3.1.1), on which practitioners tend to score in the high end of the scale, and for which a score in the lower end is rare), will end up with a lower KALPHA when a rare event is not detected (or rated in the same way) by all coders, even if the general agreement between coders is otherwise high [50]. One of the reasons that KALPHA has thus far not been widely used is that it does not form part of standard software packages, such as SPSS. For this reason, a specific KALPHA macro for SPSS [48], which can be downloaded here http://www.afhayes.com/spss-sas-and-mplus-macros-and-code.html, and a guideline written by de Swert [50] have been made available to assist researchers in its use.

\section{Discussion}

The assessment of treatment fidelity is a prerequisite for being able to distinguish between behavior change interventions where the delivered intervention was proficient 'state of the art' MI, and those where the delivered intervention was not competent MI. This is necessary in order to be able to know what conclusions may be drawn from the results of research trials. The present overview of practical recommendations on different aspects of treatment fidelity assessment in RCTs -collecting, selecting, coding, and reporting of MI fidelity- will assist researchers in assessing treatment fidelity in future studies.

However, determining whether an intervention "is MI" might not be as straightforward as it may sound. There are several reasons for this. First, the recommended thresholds for Beginning Proficiency and Competency (in MITI 3.1.1; in MITI 4.1, these are referred to as Fair and Good MI practice) are based on expert opinion, and in need of further research to establish their empirical support [14]. So far, they serve as guidance only. Second, it is frequently the case that some of these thresholds are reached, while others are not. Since certain aspects of MI practice (such as a high Empathy rating, and a low degree of MI Non-Adherent utterances, a high degree of client change talk, and a low degree of client sustain talk [13]) are perhaps more important than others, we may perhaps be justified in attaching greater weight to whether practitioners reach the recommended thresholds in respect of the MITI variables related to these aspect.

We may also have to think about how we should deal with situations in which some practitioners reach the recommended thresholds while others do not (even though all practitioners received the same amount of training). It is known that MI skill can vary substantially across practitioners (e.g. [35]). Such inter-practitioner variation in MI fidelity may also hamper discrimination between the Ml-based intervention and the control condition in research trials. In the studies conducted so far, effects have been analyzed according to the randomized groups independent 
of individual practitioners' level of treatment fidelity. However, perhaps an adjustment for 'low fidelity practitioners' in the analysis is needed, followed by a sensitivity analysis with exclusion of low fidelity practitioners.

Treatment fidelity assessment can be used to monitor the fidelity within clinical trials, but also to evaluate and supervise skill development in clinical practice at the same time. Using the MITI for supervision purposes during an RCT will help to improve practitioners' MI skill, but this may also influence the level of overall fidelity. This is something to take into consideration while evaluating an RCT. Furthermore, it may be the case that one or more practitioners fail to reach an acceptable level of MI skill while the trial is ongoing. Should such practitioners be prevented from counseling participants and receive additional training until they reach a sufficient level of MI skill? This would of course be inconsistent with a research aim what was to deliver the intervention in a manner that as closely as possible resembled actual clinical practice, and would perhaps not even be possible in implementation studies.

If future (process evaluation) studies consider measuring the fidelity of MI sessions, this could elucidate the actual fidelity level needed for MI to work and its specific effect on the outcome variable. Mediation analyses could indirectly assess the effect of $\mathrm{MI}$ on some outcome variable through a proposed mediator, thereby helping to entangle the working mechanisms of MI. As a result, implementation studies using MI in specific settings and contexts could benefit optimally from the findings of RCTs by implementing and focusing only on the 'essential components'.

In this manuscript, we advocate the use of the MITI instrument to assess treatment fidelity in order to achieve uniform reporting across RCTs, which would facilitate comparison across studies. However, several other instruments have been developed for the purposes of measuring $\mathrm{Ml}$ treatment fidelity [18]. If researchers are, for example, more interested in identifying the active mechanisms in $\mathrm{MI}$, they might consider selecting (a combination of) the following measurement tools [51]: Global Rating of MI Therapist (GROMIT) [52], the Sequential Code for Observing Process Exchanges (SCOPE) instrument [53] or the MI Skill Code (MISC) [54-56]. Even if researchers choose to use an instrument other than the MITI for assessing treatment fidelity, many of the aforementioned recommendations will still apply.

\section{Conclusion and Recommendations}

We have aimed to provide an overview of practical recommendations, available best practices, and pragmatic solutions to common problems that researchers might come across in the collection, selection, coding, and reporting of $\mathrm{Ml}$ fidelity data. We recommend certain practices in order to facilitate comparisons across studies where MI is used. For a comprehensive overview of our recommendations and considerations see box 1 . 
Box 1: Recommendations and considerations for assessing and reporting MI fidelity

\begin{tabular}{|c|c|c|c|}
\hline \multicolumn{2}{|c|}{ Aspect } & Recommendation & Potential considerations \\
\hline \multirow[b]{2}{*}{$\begin{array}{l}\text { Collecting and } \\
\text { selecting }\end{array}$} & Collection & $\begin{array}{l}\text { Audio record all sessions (anony- } \\
\text { mous) }\end{array}$ & $\begin{array}{l}\text { Is permission of both the client and } \\
\text { practitioner requested? }\end{array}$ \\
\hline & Selection & $\begin{array}{l}\text { Select a random representative } \\
\text { sample throughout the whole study } \\
\text { period. Preferably } 20 \% \text { of the study } \\
\text { sample, at least } 4 \text { conversations for } \\
\text { each practitioner involved or } 20 \\
\text { conversations per intervention group } \\
\text { when only overall results will be } \\
\text { reported }\end{array}$ & $\begin{array}{l}\text { Which MI fidelity instrument will be } \\
\text { used to assess the quality of MI? } \\
\text { Will the coding take place during or } \\
\text { at the end of the RCT? } \\
\text { Will the practitioner receive feedback } \\
\text { on his/her performance? } \\
\text { Will sufficient MI-fidelity be an entry } \\
\text { requirement for the practitioner? }\end{array}$ \\
\hline \multirow[t]{3}{*}{ Coding } & Coders & $\begin{array}{l}\text { Arrange trained coders beforehand or } \\
\text { facilitate training for (inexperienced) } \\
\text { coders and weekly meetings and } \\
\text { assess reliability prior to coding the } \\
\text { study sample }\end{array}$ & $\begin{array}{l}\text { Is there enough budget for emplo- } \\
\text { ying (trained) coders? } \\
\text { Is there enough time for inexperien- } \\
\text { ced coders to acquire competency? } \\
\text { Are there resources available for the } \\
\text { training of inexperienced coders? } \\
\text { Is comparison with a gold standard } \\
\text { from an experienced coding lab } \\
\text { considered? }\end{array}$ \\
\hline & $\begin{array}{l}\text { Inter-rater } \\
\text { reliability }\end{array}$ & $\begin{array}{l}\text { Double-code } 20 \% \text { of the sessions by } \\
\text { a second coder }\end{array}$ & $\begin{array}{l}\text { Is there enough budget for employ- } \\
\text { ing a second coder? }\end{array}$ \\
\hline & $\begin{array}{l}\text { Study specific } \\
\text { situations }\end{array}$ & $\begin{array}{l}\text { Report how specific trial aspects } \\
\text { were dealt with }\end{array}$ & $\begin{array}{l}\text { Does the intervention comprise } \\
\text { specific requirements that might } \\
\text { influence MI quality or are part of } \\
\text { the trial's fidelity? }\end{array}$ \\
\hline \multirow{3}{*}{ Reporting } & $\begin{array}{l}\text { Reporting MITI } \\
\text { results }\end{array}$ & Report raw and summary MITI results & $\begin{array}{l}\text { Does the research question focus on } \\
\text { fidelity of (1) (intervention) group } \\
\text { results, (2) all practitioners separately, } \\
\text { or (3) (a) practitioner(s) over time? } \\
\text { What level of MI is believed com- } \\
\text { petent? }\end{array}$ \\
\hline & $\begin{array}{l}\text { Reporting } \\
\text { inter-rater } \\
\text { reliability }\end{array}$ & $\begin{array}{l}\text { Report for the raw and summary } \\
\text { variables inter-rater reliability scores }\end{array}$ & $\begin{array}{l}\text { How will the results be handled of } \\
\text { both coders in case of a low reliabi- } \\
\text { lity score? }\end{array}$ \\
\hline & $\begin{array}{l}\text { Calculation } \\
\text { inter-rater } \\
\text { reliability }\end{array}$ & $\begin{array}{l}\text { Calculate KALPHA for ordinal scores } \\
\text { and ICC for behavioral counts }\end{array}$ & \\
\hline
\end{tabular}

\section{Abbreviations}

ICC: Intra Class Correlation; MI: Motivational Interviewing; MITI: Motivational Interviewing Treatment Integrity; RCT: randomized controlled trial 


\section{References}

[1] Miller WR and Rollnick S., Motivational interviewing, preparing people to change addictive behavior. New York: The Guildford Press, 1991.

[2] Rubak S, Sandbaek A, Lauritzen T and Christensen B., Motivational interviewing: a systematic review and meta-analysis. Br J Gen Pract 2005;55:305-12.

[3] Lundahl BW, Kunz C, Brownell C, Tollefson D and Burke BL., A meta-analysis of motivational interviewing: Twenty-five years of empirical studies. Res Soc Work Pract 2010;20:137-160.

[4] Lundahl B, Moleni T, Burke BL, Butters R, Tollefson D, Butler C and Rollnick S., Motivational interviewing in medical care settings: a systematic review and meta-analysis of randomized controlled trials. Patient Educ Couns 2013;93:157-68.

[5] Hettema J, Steele J and Miller WR., Motivational interviewing. Annu Rev Clin Psychol 2005;1:91-111.

[6] Burke $\mathrm{B}$, Arkowitz $\mathrm{H}$ and Menchola M., The efficacy of motivational interviewing: a meta-analysis of controlled clinical trials. J Consult Clin Psychol 2003;71:843-861.

[7] Godwin M, Ruhland L, Casson I, MacDonald S, Delva D, Birtwhistle R, Lam M and Seguin R., Pragmatic controlled clinical trials in primary care: the struggle between external and internal validity. BMC Med Res Methodol 2003;3:28.

[8] Thorpe KE, Zwarenstein M, Oxman AD, Treweek S, Furberg CD, Altman DG, Tunis S, Bergel E, Harvey I, Magid DJ and Chalkidou K., A pragmatic-explanatory continuum indicator summary (PRECIS): a tool to help trial designers. J Clin Epidemiol 2009;62:464-75.

[9] Bellg AJ, Borrelli B, Resnick B, Hecht J, Minicucci DS, Ory M, Ogedegbe G, Orwig D, Ernst $D$ and Czajkowski $\mathrm{S}$., Enhancing treatment fidelity in health behavior change studies: best practices and recommendations from the NIH Behavior Change Consortium. Health Psychol 2004;23:443-51.

[10] Moyers TB and Martin T., Therapist influence on client language during motivational inter viewing sessions. J Subst Abuse Treat 2006;30:245-51. 
[11] Lovejoy TI, Heckman TG, Suhr JA, Anderson T, Heckman BD and France CR., Telephone-administered motivational interviewing reduces risky sexual behavior in HIV-positive late middle-age and older adults: a pilot randomized controlled trial. AIDS Behav 2011;15:1623-34.

[12] Apodaca TR and Longabaugh R. Mechanisms of change in motivational interviewing: a review and preliminary evaluation of the evidence. Addiction 2009;104:705-15.

[13] Miller WR and Rollnick S., The effectiveness and ineffectiveness of complex behavioral interventions: Impact of treatment fidelity. Contemp Clin Trials 2014;37:234-241.

[14] Moyers TB, Martin T, Manuel JK, Miller WR and Ernst D., Revised Global Scales: Motivational Interviewing Treatment Integrity 3.1.1 (MITI 3.1.1) [Web Page]. Available at http://casaa.unm.edu/download/MITI3_1.pdf.

[15] Moyers TB, Martin T, Manuel JK, Hendrickson SM and Miller WR., Assessing competence in the use of motivational interviewing. J Subst Abuse Treat 2005;28:19-26.

[16] Forsberg L, Berman AH, Kallmen $H_{1}$ Hermansson $U$ and Helgason AR., A test of the validity of the Motivational Interviewing Treatment Integrity Code. Cogn Behav Ther 2008;37:1-9.

[17] Miller W and Mount K., A small study of training in motivational interviewing: does one workshop change clinician and client behavior? Behav Cogn Psychother 2001;29:457-471.

[18] Madson MB and Campbell TC., Measures of fidelity in motivational enhancement: a systematic review. J Subst Abuse Treat 2006;31:67-73.

[19] Forsberg L, Kallmen H, Hermansson U, Berman AH and Helgason AR., Coding counsellor behaviour in motivational interviewing sessions: inter-rater reliability for the Swedish Motivational Interviewing Treatment Integrity Code (MITI). Cogn Behav Ther 2007;36:162-9.

[20] Pierson HM, Hayes SC, Gifford EV, Roget N, Padilla M, Bissett R, Berry K, Kohlenberg B, Rhode $R$ and Fisher G., An examination of the Motivational Interviewing Treatment Integrity code. J Subst Abuse Treat 2007;32:11-7.

[21] Moyers TB, Manuel JK and Ernst D., Motivational Interviewing Treatment Integrity Coding Manual 4.1. Unpublished manual., 2014.

[22] Lindhardt CL, Rubak S, Mogensen O, Hansen HP, Lamont RF and Jorgensen JS. , Training in motivational interviewing in obstetrics: a quantitative analytical tool.

Acta Obstet Gynecol Scand 2014;93:698-704. 
[23] Imel ZE, Baldwin SA, Baer JS, Hartzler B, Dunn C, Rosengren DB and Atkins DC., Evaluating therapist adherence in motivational interviewing by comparing performance with standardized and real patients. J Consult Clin Psychol 2014;82:472-81.

[24] Ang DC, Kaleth AS, Bigatti S, Mazzuca SA, Jensen MP, Hilligoss J, Slaven J and Saha C., Research to encourage exercise for fibromyalgia (REEF): use of motivational interviewing, outcomes from a randomized-controlled trial. Clin J Pain 2013;29:296-304.

[25] Daeppen JB, Fortini C, Bertholet N, Bonvin R, Berney A, Michaud PA, Layat C and Gaume J., Training medical students to conduct motivational interviewing: a randomized controlled trial. Patient Educ Couns 2012;87:313-8.

[26] Broekhuizen K, Jelsma JG, van Poppel MN, Koppes LL, Brug J and van Mechelen W. Is the process of delivery of an individually tailored lifestyle intervention associated with improvements in LDL cholesterol and multiple lifestyle behaviours in people with familial hypercholesterolemia? BMC Public Health 2012;12:348.

[27] van Keulen HM, Mesters I, van Breukelen G, de Vries H and Brug J. Motivational interview ing specific fidelity scores seemed related to behavior change in an RCT with older adults. Maastricht, the Netherlands: Maastricht University, 2010.

[28] El-Mallakh P, Chlebowy DO, Wall MP, Myers JA and Cloud RN., Promoting nurse interventionist fidelity to motivational interviewing in a diabetes self-care intervention. Res Nurs Health 2012;35:289-300.

[29] Naar-King S, Outlaw A, Green-Jones M, Wright K and Parsons JT., Motivational interviewing by peer outreach workers: a pilot randomized clinical trial to retain adolescents and young adults in HIV care. AIDS Care 2009;21:868-73.

[30] Robbins LB, Pfeiffer KA, Maier KS, Ladrig SM and Berg Smith SM., Treatment fidelity of motivational interviewing delivered by a school nurse to increase girls' physical activity. J Sch Nurs 2012;28:70-8.

[31] Smith DC, Lanesskog D, Cleeland L, Motl R, Weikert M and Dlugonski D., Motivational interviewing may improve exercise experience for people with multiple sclerosis: A small randomized trial. Health Soc Work 2012;37:99-109.

[32] Seng EK and Lovejoy TI., Reliability and validity of a treatment fidelity assessment for motivational interviewing targeting sexual risk behaviors in people living with HIV/AIDS. J Clin Psychol Med Settings 2013;20:440-8. 
[33] McCarthy MM, Dickson W, Katz SD, Sciacca K and Chyun DA., Process evaluation of an exercise counseling intervention using motivational interviewing.

Appl Nurs Res 2015;28:156-62.

[34] Amrhein PC, Miller WR, Yahne CE, Palmer M and Fulcher L., Client commitment language during motivational interviewing predicts drug use outcomes.

J Consult Clin Psychol 2003;71:862-78.

[35] Forsberg L, Forsberg LG, Lindqvist $H$ and Helgason AR. Clinician acquisition and retention of Motivational Interviewing skills: a two-and-a-half-year exploratory study.

Subst Abuse Treat Prev Policy 2010;5:8.

[36] Maissi E, Ridge K, Treasure J, Chalder T, Roche S, Bartlett J, Schmidt U, Thomas S and Ismail K., Nurse-led psychological interventions to improve diabetes control: assessing competencies. Patient Educ Couns 2011;84:37-43.

[37] Noordman J, de Vet E, van der Weijden T and van Dulmen S., Motivational interviewing within the different stages of change: An analysis of practice nurse-patient consultations aimed at promoting a healthier lifestyle. Soc Sci Med 2013;87:60-67.

[38] Hettema JE, Ernst D, Williams JR and Miller KJ., Parallel processes: using motivational interviewing as an implementation coaching strategy. J Behav Health Serv Res. 2014;41:324-36.

[39] McCambridge J, Day M, Thomas BA and Strang J. Fidelity to Motivational Interviewing and subsequent cannabis cessation among adolescents. Addict Behav 2011;36:749-54.

[40] Carpenter KM, Cheng WY, Smith JL, Brooks AC, Amrhein PC, Wain RM and Nunes EV. "Old dogs" and new skills: how clinician characteristics relate to motivational interviewing skills before, during, and after training. J Consult Clin Psychol 2012;80:560-73.

[41] Bohman B, Forsberg L, Ghaderi A and Rasmussen F., An Evaluation of Training in Motivational Interviewing for Nurses in Child Health Services.

Behav Cogn Psychother 2013;41:329-343.

[42] Dewing S, Mathews C, Cloete A, Schaay N, Shah M, Simbayi L and Louw J., From research to practice: lay adherence counsellors' fidelity to an evidence-based intervention for promoting adherence to antiretroviral treatment in the Western cape, South Africa. AIDS Behav 2013;17:2935-45. 
[43] van Eijk-Hustings YJL, Daemen L, Schaper NC and Vrijhoef HJM., Implementation of Motivational Interviewing in a diabetes care management initiative in the Netherlands. Patient Educ Couns 2011;84:10-15.

[44] Hallgren KA., Computing Inter-Rater Reliability for Observational Data: An Overview and Tutorial. Tutor Quant Methods Psychol 2012;8:23-34.

[45] Moyers TB and Miller WR., Is low therapist empathy toxic?

Psychol Addict Behav 2013;27:878-84.

[46] Shrout PE and Fleiss JL., Intraclass correlations: uses in assessing rater reliability. Psychol Bull 1979;86:420.

[47] Cicchetti DV and Sparrow SA., Developing criteria for establishing interrater reliability of specific items: applications to assessment of adaptive behavior.

Am J Ment Defic 1981;86:127-37.

[48] Hayes AF and Krippendorff K., Answering the Call for a Standard Reliability Measure for Coding Data. Comm Methods Meas 2007;1:77-89.

[49] Krippendorff K., Agreement and Information in the Reliability of Coding. Comm Methods Meas 2011;5:93-112.

[50] De Swert K., Calculating inter-coder reliability in media content analysis using Krippendorff's Alpha.

Working paper: http://www.polcomm.org/wp-content/uploads/ICR01022012.pdf, 2012.

[51] Dobber J, van Meijel B, Barkhof E, Scholte Op Reimer W, Latour C, Peters R and Linszen D., Selecting an optimal instrument to identify active ingredients of the motivational interviewing-process. J Psychosom Res 2014;78:268-76.

[52] Moyers TB., Global Rating of Motivational Interviewing Therapist (GROMIT) [Web Page]. Available at http://casaa.unm.edu/download/GROMIT.pdf.

[53] Martin T, Moyers TB, Houck J, Christopher P and Miller WR., Motivational Interviewing Sequential Code for Observing Process Exchanges (MI-SCOPE). Coder's Manual [Web Page]. Available at http://casaa.unm.edu/download/scope.pdf. Accessed 18-10-2010.

[54] Glynn LH and Moyers TB., Manual for the Motivational Interviewing Skill Code (MISC), Version 1.1: Addendum to MISC 1.0 [Web Page]. Available at http://casaa.unm.edu/download/MISC_1.1_Manual.pdf. Accessed 19-10-2010. 
[55] Miller WR, Moyers TB, Ernst D and Amrhein PC Manual for the Motivational Interviewing Skill Code (MISC). Version 2.1. University of New Mexico, Center on Alcoholism, Substance Abuse, and Addictions, 2008.

[56] Moyers TB, Miller WR and Hendrickson SM., How does motivational interviewing work? Therapist interpersonal skill predicts client involvement within motivational interviewing sessions. J Consult Clin Psychol 2005;73:590-8. 


\section{Chapter 5}

Prepared for treatment? Process evaluation of a nurse-led motivational interviewing-based pre-treatment in pain rehabilitation

Mertens, V.-C. van Haastregt, J.

Verbunt, J.A.

Smeets, R.J.

Goossens, M.E.

Submitted for publication. 


\section{Abstract}

Objective: This study aims to examine the feasibility of a new Motivational Interviewing (MI) based pre-treatment (MIP) program to support patients to set realistic goals and increase their motivation for rehabilitation treatment.

Design and subjects: In this mixed methods study, data were collected among patients $(n=81)$ with chronic non-specific musculoskeletal pain who were assigned to the MIP-program in the two-armed Prepare randomized controlled trial, and nurses $(n=2)$ who conducted the program. The MIP-program consists of two individual one hour sessions conducted by a nurse according to the principles of Motivational Interviewing including empathic counselling, asking open questions, using reflection, and exploring discrepancies.

Measures: Data on patients' attendance, performance of the program according to protocol, patients' adherence, and satisfaction of patients and nurses with the program, were collected during and after the program by means of self-administered questionnaires, registration forms, interviews, and structured observation.

Results: Ninety-five percent of the target population participated in the MIP-program. Both patients and nurses reported that adherence of patients with the MIP-program was sufficient. Overall, the nurses conducted all pre-specified elements of the program; however the quality of their MI performance was below standard. Although the nurses and majority of patients were satisfied with the program, they also identified some limitations.

Conclusions: Based on this study we conclude that the attendance and adherence of participants are satisfactory, but the program is not optimally performed according to protocol. In order to further improve the feasibility of the MIP-program several recommendations for improvement are made.

\section{Clinical messages:}

- The participation of eligible patients in the nurse-led MI-based pre-treatment was high.

- Although the adherence of the participants was sufficient and nurses and patients were satisfied with the program, they indicated the need for improvement of some aspects of the intervention.

- The nurses conducted all pre-specified elements of the program, however the quality of their MI performance was below standard, therefore additional training and supervision of nurses is recommended. 


\section{Introduction}

Chronic non-specific musculoskeletal pain causes a major health burden. It occurs in approximately $10 \%$ of the general population, causing disability and detrimental mental health consequences leading to a higher level of depression and lower quality of life [1]. Furthermore, the costs for society are substantial, and even higher as the costs for heart diseases, cancer, and diabetes [2]. Multidisciplinary biopsychosocial rehabilitation programs are widely used for people with chronic low back pain [3]. A recent systematic review revealed that multidisciplinary pain rehabilitation is more effective in reducing pain and disability in patients with chronic low back pain than usual care or physical treatment [3]. In addition, there is some evidence that multidisciplinary rehabilitation improves work outcomes, compared to physical treatment. However, the effects are only small and more insight is needed in factors influencing the effectiveness of pain rehabilitation.

One possible reason for the only modest effectiveness of pain rehabilitation in patients with chronic low back pain seems to be the high levels of non-adherence and drop-out [4-6]. It is observed among patients with chronic pain, that when they start their rehabilitation treatment most patients expect their pain to be significantly reduced or even completely resolved during the rehabilitation treatment $[7,8]$. However, this expectation is not in accordance with the primary aim of the pain rehabilitation treatment, which is to decrease pain-related disability by increasing coping skills. Unresolved discrepancies between the expectations of the patient and the aims of the practitioner may result in demotivation, lack of trust in therapy, non-adherence and drop-out of the patient $[9,10]$. To prevent non-adherence and drop-out, it is important that patients have realistic expectations, set realistic goals and are motivated to adhere to their rehabilitation treatment.

A potential method to help patients to set realistic goals and to enhance their motivation for treatment is Motivational Interviewing (MI). MI is a person-centred form of counselling used to elicit and strengthen motivation for change [11]. MI aims to explore and strengthen a person's intrinsic motivation in order to enhance behavioural change [12]. MI has shown to be especially effective as pre-treatment, prior to the main treatment [13-15].

However, currently no evidence is available about the feasibility and effectiveness of such pre-treatment programs for musculoskeletal pain rehabilitation, so there is a strong need for additional research in this domain [16]. Therefore we developed a MI-based pre-treatment (MIP) program. The main goals of this MIP program are to support the patients to set realistic goals for pain rehabilitation treatment and to increase their motivation for treatment. Furthermore, the program aims to improve adherence to the subsequent pain rehabilitation treatment, prevent treatment drop-out, and improve social participation.

The effects of this trial [17] are currently being assessed in a randomized controlled trial. Alongside this trial a process evaluation is performed to assess the feasibility of the MIP program. A process evaluation can help to correctly interpret the results of a trial because it provides an understanding of how and why an intervention was effective or ineffective [18]. Furthermore, it can provide suggestions for further optimising the intervention $[19,20]$. The current paper describes the results of the process evaluation of the MIP-program. As recommended by Oakley 
and colleagues the process evaluation has been performed prior to analysing the effects of the MIP-program in order to prevent possible bias related to knowledge of the outcome of the intervention [18].

The objectives of this study are to assess

(1) patients' attendance to the MIP-program;

(2) the performance of the program according to protocol;

(3) patients' adherence to the program; and

(4) patients' and nurses' satisfaction with the program.

\section{Methods}

\section{The MIP program}

The MIP program is conducted by a registered nurse and consists of two individual interactive sessions lasting 45 to 60 minutes. The two nurses who conducted the program are certified $\mathrm{Ml}$-coaches who received additional training to update their knowledge of and experience with $\mathrm{MI}$ in the context of chronic pain rehabilitation [21-28]. Furthermore, the nurses participated in three supervision sessions during the 1.5 year intervention period, to discuss their performance during the MIP sessions.

The period between the first and second session had to vary between one and four weeks, and depended on the availability and preferences of nurses and patients. Both sessions were conducted according to the principles of Motivational Interviewing including empathic counselling style, asking open questions, using reflection, and exploring discrepancies [29]. The MIP program is described in detail in a protocol (manual) for the nurses, which is available on request.

\section{First session}

The first session focused on daily activities in which the patient feels impaired due to chronic pain. Activities were based on the 'Activities and participation' taxonomy of the International Classification of Functioning, Disability, and Health (ICF model) and consisted of topics such as self-care and mobility as well as self-chosen undefined activities [30].

The sessions consisted of six elements. First, the patient's life situation was explored. Second, the patient was invited to select relevant daily activities printed on ten paper cards. Third, the patient was invited to sort the selected activities according to the level of disability he or she perceived when performing each activity. Fourth, the patient was asked to sort the selected activities based on the perceived importance of each activity in their daily life. Fifth, the patient was invited to select activities which he/she considered both important and difficult to perform owing to his/ her disability. The patient was asked to sort the selected activities from high to low to reflect his/ 
her confidence in reducing the level of disability in those activities. Sixth, the nurse reflected on the previous five steps and provided feedback following the principles of MI. The nurse discussed the main aims and characteristics of pain rehabilitation treatment and the importance of setting realistic goals. Furthermore, strategies to improve confidence of the patient in favourable rehabilitation outcomes were explored. For example, the patient was invited to think about different scenarios that could increase his/her confidence in performing the selected activities. As step six could not be completed for all selected activities within one session, the patient received a homework assignment at the end of the first session. It was estimated that making the homework assignment would take between 20 and 40 minutes. The assignment consisted of choosing two undiscussed activities from the selected activities. In addition, the patient received a small booklet in which to answer nine questions about the potential for change and his/ her confidence in change during the pain rehabilitation treatment. If a patient had a partner, the patient was encouraged to discuss the answers to the questions with his/her partner.

\section{Second session}

The second session started with discussing the homework assignment. Next, step six was completed by discussing the remaining selected activities (if applicable). Finally, the nurse discussed potential realistic goals for pain rehabilitation treatment with the patient based on the results of the first session, the homework assignment and the discussion of the remaining selected activities.

\section{Design}

This is a mixed method study with a triangulation design, in which quantitative and qualitative data are combined in order to gain optimal insight in the feasibility of the MIP-program. Data were gathered during and after the implementation of the MIP-program in two hospitals in the South of the Netherlands between January 2012 and September 2014.

\section{Participants}

Patients

Data were collected among 81 patients with chronic non-specific musculoskeletal pain who were assigned to the MIP-program in the two-armed randomised controlled trial [17] in which the effects of the program are being assessed. Patients were included in the study between January 2012 and June 2013. Patients were recruited at the outpatient rehabilitation medicine departments of an university hospital and a regional hospital in the south of the Netherlands. Inclusion criteria were: age between 18 and 65, non-specific chronic musculoskeletal pain for longer than 3 months, chronic pain syndrome not attributable to medical pathology (e.g. infection, tumour, osteoporosis, fracture, structural deformity or inflammatory disorder), and adequate literacy to complete assessment measures. In addition (according to the usual intake procedure for pain rehabilitation treatment) the patients should have an initial medium to high level of motivation for pain rehabilitation treatment as judged by a consultant in rehabilitation medicine during intake. Exclusion criteria were pregnancy, surgery planned for the near future, involvement in a litigation 
procedure, and a psychiatric disease that is expected to interfere with the rehabilitation treatment (e.g. psychosis or personality disorder) as assessed by a rehabilitation medicine consultant.

\section{Nurses}

The MIP program was conducted by two registered nurses, who have worked in the field of pain rehabilitation for over fifteen years. Both nurses were certified MI coaches with four years of experience.

\section{Data collection}

Data on the feasibility of the MIP program were collected by means of:

1) a questionnaire for patients consisting of seven open and thirty-one closed questions which the patients had to administer after the second session of the program;

2) a registration form for nurses consisting of eight open and seventeen closed questions to be completed after each session of the MIP program. Therein, satisfaction with the session had to be completed after each session by the nurse. The registration form consisted furthermore of three additional questions regarding the homework assignment to be completed after the second session;

3) a short telephone interview with patients who decided not to complete the MIP program; and 4) structured observation of audio tapes of sessions of the MIP program. In addition, background characteristics of the patients (age, gender, pain etiology, pain duration) were collected by means of a self-administered questionnaire which was part of the previously mentioned trial in which the effectiveness of the MIP program is evaluated.

The process outcomes measured in this study are based on the key elements of process evaluation presented by Saunders and colleagues [19], and are summarized in Table 1.

\section{Attendance}

Attendance (or reach) is assessed by measuring the proportion of the intended target population that participated in the MIP program, the number of patients who did not complete the intervention, and their reasons for non-attendance.

\section{Performance according to protocol}

Performance according to protocol is assessed by measuring dose delivered and fidelity of the program. Dose delivered consists of assessing whether the planned sessions were actually performed within the planned timeframe and included all six pre-specified program elements. In addition, reasons for deviation from protocol were assessed. Fidelity (or integrity) of MIP program was assessed by means of the reliable and validated "Motivational Interviewing Treatment Integrity" (MITI) scale [31]. The MITI scale is a structured observation scale which evaluates the MI performance of the nurses who conduct the program. In our study the observations are based on audiotapes of the sessions of the MIP program. All sessions were audiotaped and 32 sessions $(20 \%)$ were randomly selected to be analysed with the MITI scale. Five summary scores are calculated based on the MITI scale: MI-spirit, percentage of open questions, percentage of complex 
reflections, percentage of $\mathrm{Ml}$-adherent responses, and reflection to question ratio. For each score a threshold is set for beginning proficiency. A score below this threshold indicates that the MI element is not applied according to the professional standards as set by the developers of the scale [31]. Additional details on the assessment of fidelity of the MIP program are reported elsewhere [32].

\section{Adherence to the program}

Adherence (or exposure) refers to the patient's active engagement in the intervention. Both nurses and patients were asked to rate the patient's engagement in both sessions on a 5-point Likert scale, ranging from 'low' (1) to 'excellent' (5). In addition participants had to indicate whether they worked on the homework assignment (answer options: 'yes', 'partly', 'no') and an open question regarding reasons of partial or non-completion of the homework assignment. Furthermore, the patients were asked to indicate how much time (in minutes) they had spent on it. Furthermore, nurses were asked to judge the patient's adherence to the homework assignment. This was measured on a three-point Likert-scale ranging from 'good', to 'moderate' or 'not' with the option to elaborate upon the answer in an open text field.

\section{Satisfaction with the program}

Satisfaction with the program was assessed by measuring the patients' and nurses' overall satisfaction with the program (rated on a scale from 1 to 10). Patients rated this after the second session, and nurses after each session. In addition the nurses and patients were asked to report strengths and limitations of the program. Nurses' could give answers on open questions. Patients were asked to report upon yes/no items: Eight yes/no items describing targeted benefits of the program such as knowledge regarding the rehabilitation treatment, plans to exercise more, and plans to start the rehabilitation treatment, their opinion on the homework assignment (measured by three yes/no items regarding the amount, pleasure, and insights due to the homework assignment), and suggestions for improvement of the program by a closed question whether improvements of the pre-treatment are necessary, and the option to elaborate upon this by answering an open question regarding which improvements they recommend.

Table 1 presents an overview of the process outcomes and the way the data are collected.

\section{Ethical considerations}

The study was approved by the Medical Ethical Committee of University Hospital Maastricht and Maastricht University. The trial is registered in a public trial registry (Nederlands Trial Register NTR3065).

\section{Data analysis}

The quantitative data from the questionnaires and registration forms were analysed by means of descriptive statistics using Statistical Software Package for Social Sciences (SPSS), version 21 (SPSS Inc., Chicago, IL). Qualitative data, resulting from answers to open questions were categorised in order to identify relevant themes by two independent raters. 
Table 1: Process outcomes and measurement instruments

\begin{tabular}{|c|c|c|c|c|c|}
\hline \multirow{3}{*}{$\begin{array}{l}\text { Process } \\
\text { outcome }\end{array}$} & \multirow{3}{*}{$\begin{array}{l}\text { Operatio- } \\
\text { nalization }\end{array}$} & \multicolumn{4}{|c|}{ Measurement Instruments } \\
\hline & & \multicolumn{2}{|c|}{ Patients } & \multicolumn{2}{|c|}{ Nurses } \\
\hline & & $\begin{array}{l}\text { Self-adminis- } \\
\text { tered questi- } \\
\text { onnaire }\end{array}$ & Short interviews & $\begin{array}{l}\text { Registration } \\
\text { form }\end{array}$ & $\begin{array}{l}\text { Structured } \\
\text { observation }\end{array}$ \\
\hline \multirow[t]{2}{*}{$\begin{array}{l}\text { Attendance } \\
\text { (reach) }\end{array}$} & $\begin{array}{l}\text { Attendance of } \\
\text { sessions }\end{array}$ & & & $x$ & \\
\hline & $\begin{array}{l}\text { Reasons for } \\
\text { non-attendance }\end{array}$ & & $x$ & & \\
\hline \multirow{4}{*}{$\begin{array}{l}\text { Performance } \\
\text { according to } \\
\text { protocol (dose } \\
\text { delivered and } \\
\text { fidelity) }\end{array}$} & $\begin{array}{l}\text { Duration of the } \\
\text { sessions }\end{array}$ & & & $x$ & \\
\hline & $\begin{array}{l}\text { Performance of } \\
\text { the six pre-speci- } \\
\text { fied program } \\
\text { elements }\end{array}$ & & & $\mathrm{x}$ & \\
\hline & $\begin{array}{l}\text { Reasons for } \\
\text { deviations from } \\
\text { protocol }\end{array}$ & & & $\mathrm{x}$ & \\
\hline & $\begin{array}{l}\text { Reasons for } \\
\text { deviations from } \\
\text { protocol }\end{array}$ & & & $\mathrm{x}$ & $x$ \\
\hline \multirow[t]{2}{*}{$\begin{array}{l}\text { Adherence } \\
\text { (exposure) }\end{array}$} & $\begin{array}{l}\text { Patients' en- } \\
\text { gagement in the } \\
\text { sessions }\end{array}$ & $x$ & & $\mathrm{x}$ & \\
\hline & $\begin{array}{l}\text { Adherence to } \\
\text { homework } \\
\text { assignment }\end{array}$ & $\mathrm{x}$ & & $\mathrm{x}$ & \\
\hline \multirow[t]{5}{*}{ Satisfaction } & $\begin{array}{l}\text { Overall satisfac- } \\
\text { tion with the } \\
\text { program }\end{array}$ & $\mathrm{x}$ & & $\mathrm{x}$ & \\
\hline & $\begin{array}{l}\text { Strengths and } \\
\text { weaknesses of } \\
\text { the program }\end{array}$ & $x$ & & $\mathrm{x}$ & \\
\hline & $\begin{array}{l}\text { Perceived } \\
\text { benefits of the } \\
\text { program }\end{array}$ & $x$ & & & \\
\hline & $\begin{array}{l}\text { Opinion on } \\
\text { homework } \\
\text { assignment }\end{array}$ & $x$ & & & \\
\hline & $\begin{array}{l}\text { Suggestions for } \\
\text { improvement of } \\
\text { the program }\end{array}$ & $x$ & & & \\
\hline
\end{tabular}




\section{Results}

\section{Background characteristics and response}

Table 2 shows the demographic characteristics of the 81 patients allocated to MIP. Mean age of the participants is 47 years (ranging from 22 to 73). Seventy percent of the patients are female and in about one third of the patients' pain aetiology is related to fibromyalgia. Mean pain duration is 11 years and ranges from 5 months to 30 years.

Table 2: Background characteristics participants in MIP program $(n=81)$

\begin{tabular}{|l|l|l|}
\hline Characteristic & \multicolumn{2}{l|}{} \\
\hline Mean age (SD) & 46.8 & $(11)$ \\
\hline N (\%) female & 57 & $70,4 \%$ \\
\hline Pain aetiology: & & \\
\hline - Fibromyalgia & 28 & 34,6 \\
\hline $\begin{array}{l}\text { O Other non-specific musculoskeletal chronic } \\
\text { pain syndromes }\end{array}$ & 53 & 65,4 \\
\hline Mean pain duration in years (SD) & 11.0 & $(9.6)$ \\
\hline
\end{tabular}

Fifty-eight (75\%) of the 77 patients who participated in at least one MIP session completed the questionnaire after the MIP program. All patients who withdrew from the program participated in the short interviews. Nurses' registration forms were available in 66 of the 77 first sessions and 64 of the 74 second MIP sessions (see also Figure 1).

\section{Attendance (reach)}

Seventy-seven of the 81 patients (95\%) allocated to the MIP participated in the first MIP session, and 74 patients (91.3\%) participated in both sessions (Figure 1). Reasons for non-attendance are described in Figure 1.

\section{Performance according to protocol (dose delivered and fidelity)}

The first session took an average of 48 minutes (ranging from 35 to 70 minutes) and the second also lasted on average 48 minutes (ranging from 35 to 65 minutes). Twenty-six of the in total 130 sessions lasted less than 45 minutes and four sessions lasted more than 60 minutes. The nurses reported that they addressed all six pre-specified elements described in the protocol in almost all sessions: in $96 \%$ of the first and 100\% of the second sessions. Reasons for not addressing all elements were: content had to be changed because two sessions had to be combined into one due to restricted availability of the patient $(n=1)$, the session took place via remote counselling by webcam $(n=1)$, and the session ended prematurely because the patient expressed serious emotions unrelated to the intervention preventing further continuation of the session $(n=1)$ (this 
Figure 1: Flow chart participants MIP-program

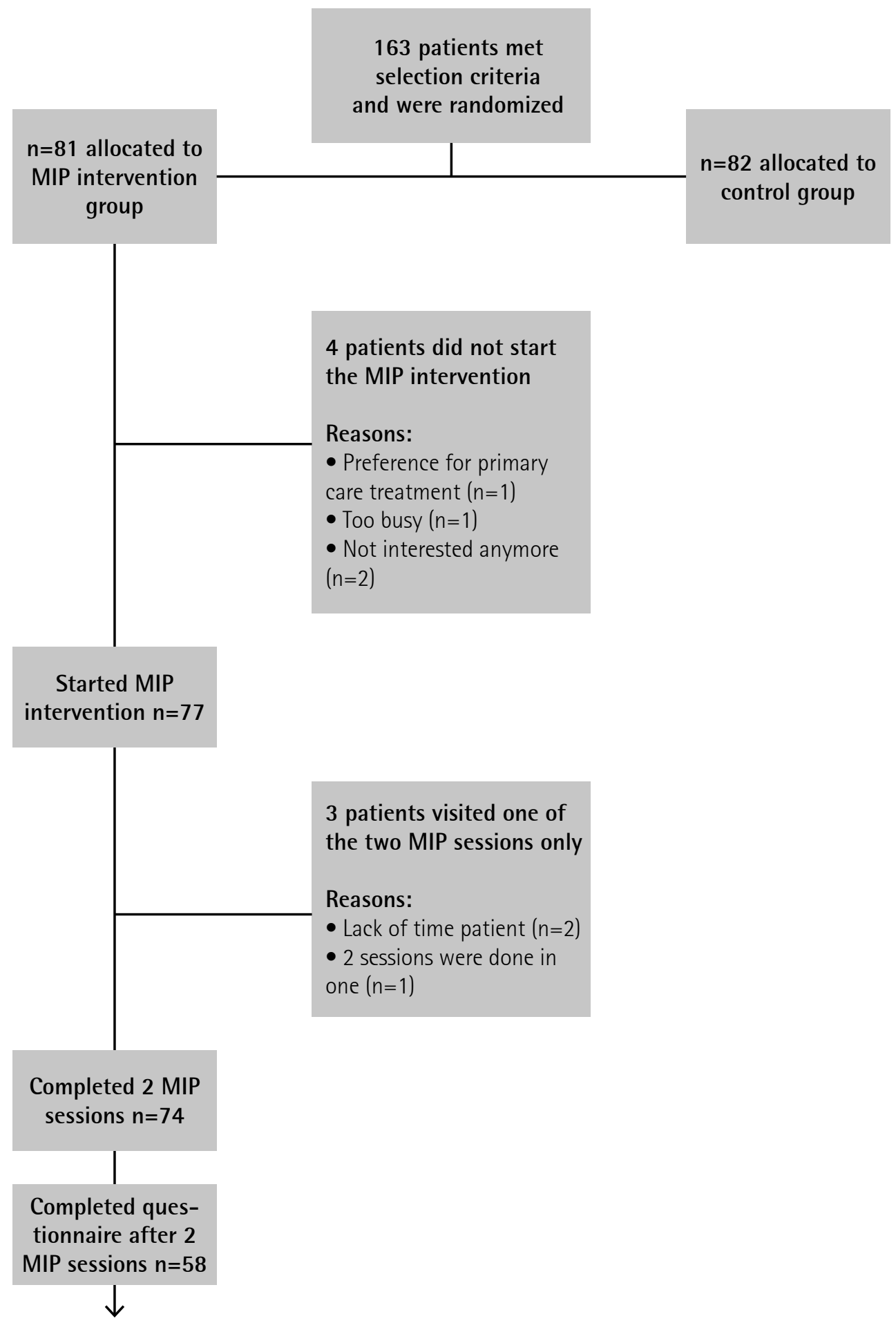


session took eight minutes and was therefore considered an outlier and was excluded from the analysis).

The fidelity observations of the sessions with the MITI scale, revealed that the MI-spirit was 3.41 (threshold 3.50), reflection to question ratio was 1.01 (threshold 1.00), percentage of open questions was $40 \%$ (threshold 50\%), percentage of MI-adherent response was $37.7 \%$ (threshold 90\%), and complex reflections was $23 \%$ (threshold $40 \%$ ). Thus, the threshold for beginning proficiency was met only for the reflection to question ratio [32].

\section{Adherence to the MIP program (exposure)}

Nurses rated the engagement of the patients as good to excellent in $87 \%$ of the first sessions and in $81 \%$ of the second sessions, whereas patients rated their own engagement as good to excellent in $46 \%$ of the available responses for both sessions.

Eighty-eight percent of the patients reported that they completed their homework assignment and that it took them on average 32 minutes (range 5 to 130; SD = 20 minutes) to complete it. Sixty-eight percent of the 63 patients with a partner reported to have completed the homework assignment together with their partner. According to the nurses, 73\% of the patients had worked intensively on their homework.

\section{Satisfaction}

The nurses rated their satisfaction with the program with an average of 7.3 out of 10 (range 5 to $9 ;$ SD .78). The nurses reported as strengths of the first session that the patients were active and engaged, gained new insights, and discussed their problems with disabilities in daily life. The nurses reported as strengths of the second session that the patients gained new or deeper insights and became more aware of their disabilities and possibilities. The nurses reported as most prominent weakness of the first and the second session that they could have deeper explored the patients' views. Additionally, as most prominent weakness of the second session, the nurses reported that the homework was not completed by everyone.

Overall, patients graded the intervention with an average of 8.2 (SD 0.9; range 6-10). About two thirds $(n=38)$ of the patients reported as strengths of the program that they had a good conversation with the nurse, that the nurse listened to what they had to say and/or that they felt acknowledged by the nurse.

Most patients $(n=25)$ did not report any weaknesses of the program. Eleven percent $(n=5)$ mentioned as limitation of the program, that the subsequent pain rehabilitation trajectory was not clear to them. Other responses contained comments regarding the amount of questionnaires related to the participation in the trial and the homework assignment. The patient's opinions regarding the homework assignment are presented in Table 3 . The majority of patients said that the amount of time needed to complete the homework was fine and that they enjoyed doing it $(81 \%)(n=45)$. Forty-five percent reported that they did not learn a lot while completing the homework assignment $(n=25)$. 
Table 3: Patients' opinions regarding the homework assignment $(n=56)$

\begin{tabular}{|c|c|c|}
\hline $\begin{array}{l}\text { My opinions regarding the homework assignment of the MIP } \\
\text { program }\end{array}$ & $\mathrm{N}$ & $(\%)$ \\
\hline The amount of time needed to complete the homework was fine & 45 & 81 \\
\hline I enjoyed doing the homework assignment & 45 & 81 \\
\hline I did learn a lot while completing the homework assignment & 25 & 45 \\
\hline
\end{tabular}

The perceived benefits of the MIP program as reported by the patients are presented in Table 4. Seven of the eight targeted benefits were confirmed by $64 \%$ to $91 \%$ of the participants. Only one targeted benefit (the actual uptake of exercise) was confirmed by less than half of the participants.

Table 4: Benefits of pre-treatment according to patients $(n=58)$

\begin{tabular}{|l|c|c|}
\hline After attending the sessions of the MIP program & N & (\%) \\
\hline I know what the aim of pain rehabilitation is & 54 & 91 \\
\hline I started thinking about activities I want to reassume & 54 & 91 \\
\hline I made a deliberate choice for a treatment & 51 & 86 \\
\hline I developed plans on what to change during rehabilitation treatment & 49 & 83 \\
\hline I started thinking about my pain-related disabilities & 47 & 80 \\
\hline I made plans to exercise more & 45 & 76 \\
\hline I have formed a clear picture about what to expect from & 38 & 64 \\
\hline rehabilitation treatment & & \\
\hline I started exercising & 27 & 46 \\
\hline
\end{tabular}

Almost one third of the patients $(29 \%, n=17)$ made recommendations for improvement of the MIP program. The following recommendations were made: improve the clarity of the questions in the homework assignment $(n=7)$, provide more information on rehabilitation treatment and/ or contact with fellow sufferers $(n=4)$, increase the number and/or duration of sessions $(n=2)$, present the MIP program later in the treatment trajectory $(n=2)$, provide a better explanation of the aim of the MIP program $(n=1)$, and provide a better explanation of the importance of the homework assignment $(n=1)$. 


\section{Discussion}

This study examined the feasibility of a Ml-based pre-treatment program for patients with chronic non-specific musculoskeletal pain. The results of this study show that the attendance of the two program sessions was high: $91 \%(n=74)$ of the target population participated in both sessions. Regarding the performance of the program according to protocol, the nurses reported that they addressed al six pre-specified program elements in almost all sessions. However, observation of five fidelity aspects revealed that for four of the five $\mathrm{MI}$ aspects the threshold for beginning MI proficiency was not reached, suggesting that the MI skills of the nurses were insufficient. In addition, there was a clear discrepancy between the patient engagement as rated by nurses and patients. The nurses rated the engagement of the patients with the MIP program much higher than the patients themselves (87\% for the first session and $81 \%$ for the second session respectively $95 \%$ versus $46 \%$ ). It seems that the nurses overrated the engagement of the patients or had other expectations than the patients about the targeted level of engagement. Both patients and nurses rated the MIP program favourably (8.2 and 7.3 respectively). Nevertheless, they reported some limitations of the program including a lack of clarity of the homework assignment (as reported by the patients) and the fact that the nurses were not always able to elaborate enough on the views of the patients (as reported by the nurses).

Since, to our knowledge, this is the first process evaluation in the field of musculoskeletal pain that has evaluated an Ml-based pre-treatment program, we cannot compare our results to other studies in the same domain. However, the high levels of satisfaction of patients with the MIP intervention identified in our study are in line with studies assessing MI in intervention programs for other patient groups, such as patients with inflammatory bowel disease [33], highly distressed patients with rheumatoid arthritis [34], stroke patients in an acute care setting [35] and patients with overweight and obesity [36]. With regard to the application of Ml elements the results of our study are also in line with the findings of Pollak and colleagues who reported that the application of Ml elements was low in their MI program for patients with overweight and obesity [36]. Although the nurses were trained for the MIP program and were trained and experienced MI coaches, most of their Ml skills appeared to be below the threshold of beginning MI proficiency [31]. Previous studies on MI programs have also indicated that some MI skills may be difficult to acquire (e.g.[37]).

\section{Limitations}

The current process evaluation has some limitations. First, the majority of data was gathered by means of self-report by nurses and patients. Self-reporting of data can cause bias due to social desirability. In order to reduce bias, we informed participants that the information would not 
be used to inform health care providers (relevant for the patients involved) or the management (relevant for the nurses involved). Second, one third of the patients recommended improvements for the MIP intervention. It would have been of additional value to elaborate on this by performing focus groups with both patients and nurses. Thirdly, the majority of measurement instruments was developed by the researchers of this study in order to optimally tailor the questions to the specific setting and program under study, which is customary in process evaluations. As a consequence it is not clear whether these instruments are reliable and valid. In order to increase the reliability and validity, all measurement instruments were pilot tested among a small group of patients and improved if necessary.

Despite these limitations, the strengths of the current study include an extensive and mixed data collection from the patients' and nurses' perspective as well as a systematic approach of the process evaluation based on a widely-used framework and key elements of Saunders and colleagues [19].

\section{Conclusion}

Based on this study we conclude that the attendance of patients with chronic pain to the new MIP program is high, and the adherence is sufficient. However, the MIP program is not optimally performed according to protocol. In order to further improve the feasibility of the MIP-program several recommendations for improvement are made. First, we propose to intensify the training of nurses (or other professionals) who perform the MIP program in order to increase their MI skills and learn them to elaborate more on the views of the patients. Second, we recommend that the nurses are supervised by an experienced Ml trainer on a regular basis to discuss their experiences and the problems they encounter when conducting the program. Third, we recommend to improve the clarity of the questions in the homework assignment.

\section{List of abbreviations}

\section{Abbreviation}

\begin{tabular}{|l|l|}
\hline MI & Motivational Interviewing \\
\hline MIP & Motivational Interviewing-based pre-treatment \\
\hline
\end{tabular}




\section{References}

[1] Andersen LN, Kohberg M, Juul-Kristensen B, Herborg LG, Søgaard K, Roessler KK., Psychosocial aspects of everyday life with chronic musculoskeletal pain: A systematic review. Scand J Pain. 2014;5(2):131-48.

[2] Gaskin DJ, Richard P., The economic costs of pain in the United States.

J Pain. 2012;13(8):715-724.

[3] Kamper SJ, Apeldoorn AT, Chiarotto A, Smeets RJ, Ostelo RW, Guzman J, et al., Multidisciplinary biopsychosocial rehabilitation for chronic low back pain. Cochrane Database Syst Rev. 2014;9:CD000963.

[4] Rainville J, Ahern DK, Phalen L., Altering beliefs about pain and impairment in a functionally oriented treatment program for chronic low back pain. Clin J Pain. 1993;9(3):196-201.

[5] Peters J, Large RG, Elkind G., Follow-up results from a randomised controlled trial evaluating in- and outpatient pain management programmes. Pain. 1992;50(1):41-50.

[6] Bendix AE, Bendix T, Haestrup C, Busch E., A prospective, randomized 5-year follow-up study of functional restoration in chronic low back pain patients. Eur Spine J. 1998;7(2):111-119.

[7] Petrie KJ, Frampton T, Large RG, Moss-Morris R, Johnson M, Meechan G., What do patients expect from their first visit to a pain clinic? Clin J Pain. 2005;21(4):297-301.

[8] Verbeek J, Sengers MJ, Riemens L, Haafkens J., Patient expectations of treatment for back pain: a systematic review of qualitative and quantitative studies.

Spine (Phila Pa 1976). 2004;29(20):2309-2318.

[9] Sloots M, Scheppers EF, Van de Weg FB, Dekker JH, Bartels EA, Geertzen JH, et al., Higher drop-out rate in non-native patients than in native patients in rehabilitation in The Netherlands. Int J Rehabil Res. 2009;32(3):232-237.

[10] Sloots M, Dekker JH, Pont M, Bartels EA, Geertzen JH, Dekker J., Reasons of drop-out from rehabilitation in patients of Turkish and Moroccan origin with chronic low back pain in The Netherlands: a qualitative study. J Rehabil Med. 2010;42(6):566-573. 
[11] Miller WR, Rollnick S., Ten things that motivational interviewing is not. Behav and Cogn Psychoth. 2009;37(2):129-40.

[12] Rollnick S, Miller WR, Butler C., Motivational Interviewing for Health Care Professionals. New York, NY, USA: Guilford Press; 2007.

[13] Burke BL, Arkowitz H, Menchola M., The efficacy of motivational interviewing: a meta-analysis of controlled clinical trials. J Consult Clin Psychol. 2003;71(5):843-861.

[14] Hettema J, Steele J, Miller WR., Motivational interviewing. Annu Rev Clin Psychol. 2005;1:91-111.

[15] Lundahl B, Burke BL., The effectiveness and applicability of motivational interviewing: A practice-friendly review of four meta-analyses. J Clin Psychol. 2009;65(11):1232-45.

[16] Chilton R, Pires Yfantouda R, Wylie M., A systematic review of motivational interviewing within musculoskeletal health. Psychol Health Med. 2012;17(4):392-407

[17] Mertens VC, Goossens ME, Verbunt JA, Koke AJ, Smeets RJ., Effects of nurse-led motivational interviewing of patients with chronic musculoskeletal pain in preparation of rehabilitation treatment (PREPARE) on societal participation, attendance level, and cost-effectiveness: study protocol for a randomized controlled trial. Trials. 2013;14(1):90.

[18] Oakley A, Strange V, Bonell C, Allen E, Stephenson J., Process evaluation in randomised controlled trials of complex interventions. Br Med J. 2006;332(7538):413-416.

[19] Saunders RP, Evans MH, Joshi P., Developing a process-evaluation plan for assessing health promotion program implementation: a how-to guide.

Health Promot Pract. 2005;6(2):134-47.

[20] Platt S, Gnich W, Rankin D, Ritchie D, Truman J, Backett-Milburn K., Applying process evaluation: Learning from two research projects. In: Thorogood M, Coombes $Y$, editors. Evaluating health promotion: Practice and methods. New York: Oxford University Press; 2004. p. 73-89.

[21] Söderlund LL, Madson MB, Rubak S, Nilsen P., A systematic review of motivational interviewing training for general health care practitioners. Patient Educ Couns. 2011;84(1):16-26.

[22] Myers SS, Phillips RS, Davis RB, Cherkin DC, Legedza A, Kaptchuk TJ, et al., Patient expectations as predictors of outcome in patients with acute low back pain.

J Gen Intern Med. 2008;23(2):148-53. 
[23] Miller WR, Moyers TB., Eight stages in learning motivational interviewing. J Teach Addict. 2007;5(1):3-17.

[24] Miller W, Yahne C, Moyers T, Martinez J, Pirritano M., A randomized trial of methods to help clinicians learn motivational interviewing. J Consult Clin Psychol. 2004;72(6):1050-62.

[25] Madson MB, Loignon AC, Lane $C$., Training in motivational interviewing: a systematic review. J Subst Abuse Treat. 2009;36(1):101-109.

[26] Lane $C$, Hood K, Rollnick S., Teaching motivational interviewing: Using role play is as effective as using simulated patients. Med Educ. 2008;42(6):637-644.

[27] Söderlund LL, Nilsen P, Kristensson M., Learning motivational interviewing: Exploring primary health care nurses' training and counselling experiences. Health Educ J. 2008;67(2):102-109.

[28] Moyers TB, Manuel JK, Wilson PG, Hendrickson SML, Talcott W, Durand P., A Randomized Trial Investigating Training in Motivational Interviewing for Behavioral Health Providers. Behav Cogn Psychother. 2008;36(02):149-162.

[29] Miller WR, Rollnick S., Motivational Interviewing: Preparing People for Change. (Second Ed.) ed. New York: The Guilford Press; 2002.

[30] Kostanjsek N., Use of The International Classification of Functioning, Disability and Health (ICF) as a conceptual framework and common language for disability statistics and health information systems. BMC Public Health. 2011;11(Suppl 4):S3.

[31] Moyers TB, Martin T, Manuel JK, Miller WR, Ernst D., Revised Global Scales: Motivational Interviewing Treatment Integrity 3.1.1 (MITI 3.1.1) 2010.

Available from: http://casaa.unm.edu/download/MITI3_1.pdf.

[32] Mertens VC, Forsberg L, Verbunt JA, Smeets R, Goossens ME., Treatment fidelity of a nurse-led Motivational interviewing-based pre-treatment in pain rehabilitation. J Behav Health Serv Res. 2015.

[33] Mocciaro F, Di Mitri R, Russo G, Leone S, Quercia V., Motivational interviewing in inflammatory bowel disease patients: a useful tool for outpatient counselling. Dig Liver Dis. 2014;46(10):893-897. 
[34] Vriezekolk JE, Geenen R, van den Ende CHM, Slot H, van Lankveld WGJM, van Helmond T. , Behavior change, acceptance, and coping flexibility in highly distressed patients with rheumatic diseases: feasibility of a cognitive-behavioral therapy in multimodal rehabilita tion. Patient Educ Couns. 2012 May;87(2):171-7.

[35] Byers AM, Lamanna L, Rosenberg A., The Effect of Motivational Interviewing After Ischemic Stroke on Patient Knowledge and Patient Satisfaction With Care: A Pilot Study. J Neurosci Nurs. 2010;42(6):312-22.

[36] Pollak KI, Alexander SC, Tulsky JA, Lyna P, Coffman CJ, Dolor RJ, et al., Physician empathy and listening: associations with patient satisfaction and autonomy. J Am Board Fam Med. 2011;24(6):665-672.

[37] Forsberg L, Forsberg LG, Lindqvist $H$, Helgason AR., Clinician acquisition and retention of Motivational Interviewing skills: a two-and-a-half-year exploratory study. Subst Abuse Treat Prev Policy. 2010;5:8. 


\section{Chapter 6}

Prepared for treatment? Effectiveness of a nurse-led Motivational Interviewing based pre-rehabilitation-treatment for patients with non-specific chronic pain: a randomized controlled trial.

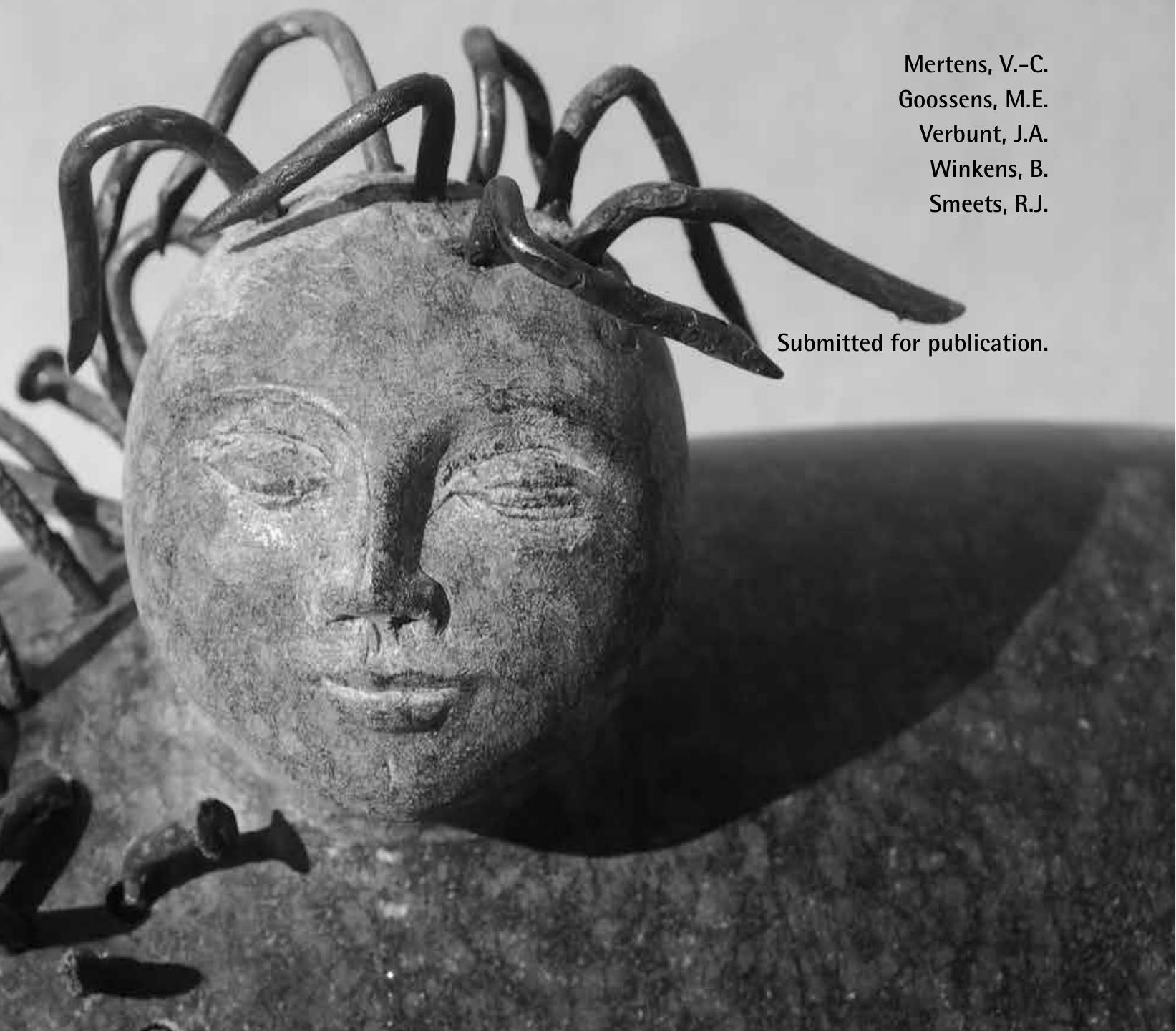




\section{Abstract}

The aim of this study was to evaluate whether a nurse-led Motivational Interviewing-based pre-treatment (MIP) is more effective than an education-based pre-treatment (UC) for patients with chronic non-specific pain undergoing pain rehabilitation treatment. This parallel-group randomized controlled trial recruited 163 patients ( $n=82$ MIP; $n=81$ UC) who remained blinded for group assignment. Pre-treatment took place at two rehabilitation medicine departments in the Netherlands. MIP was delivered by nurses in two sessions to enhance patients' motivation for rehabilitation treatment as well as to identify treatment goals. The UC condition received nurse-led pain education in two sessions. The primary outcome of social participation and the secondary outcomes of pain-related disability and treatment dropout were measured at baseline, after the pre-treatment, at the start, at the end, and six months after pain rehabilitation treatment. Linear mixed models were applied according to the intention-to-treat principle, and a per-protocol analysis was done for those who completed the pre-treatment and the subsequent pain rehabilitation treatment. The results showed no significant differences between groups in the intention-to-treat analyses. Per-protocol analyses showed similar results except for a significant difference in terms of pain-related disability favoring MIP. Moderation analysis showed that MIP is effective for improving social participation and decreasing pain-related disability in patients with a localized pain problem but not in patients with fibromyalgia. More research is needed into the effectiveness for patients with chronic pain and into Ml skills training, especially in nurse-led care.

Trial registration: Netherlands Trial register NTR3065.

Summary: Overall, Ml-based pre-treatment is not more effective as compared with pain education for improving social participation. Moderation analyses showed that MIP is especially effective for localized pain problems. 


\section{Introduction}

Chronic non-specific musculoskeletal pain is a highly prevalent health condition affecting the daily life functioning of individuals and also has a considerable impact on patients' social environment and on society as a whole $[8,17,25]$. Pain rehabilitation treatments used to help patients regain normal daily life functioning are predominantly bio-psychosocial [24]. These approaches are multidisciplinary in nature and aim to restore functioning and enhance pain management $[44,57]$. Moderate effectiveness of multidisciplinary pain rehabilitation has been shown for patients with chronic low back pain [23]. Effectiveness could be negatively influenced by adherence problems, which are reported in varying numbers $[5,37,41]$. Adherence problems can often be explained by unmet expectations of patients $[38,47,48]$. Most patients with chronic pain hope for pain relief. Although pain rehabilitation treatment can help improve patients' functioning and social participation, it does not specifically address and achieve reduction of pain [52].

An approach that may be able to increase both adherence to and motivation for pain rehabilitation is Motivational Interviewing (MI). MI is a person-centered form of counseling designed to elicit and strengthen motivation for change [35]. MI has been shown to be effective in a broad range of health behaviors (e.g. physical exercise) and settings (e.g. somatic care and psychiatric care) $[9,22,29,30,42]$. Evidence from systematic reviews shows that $\mathrm{MI}$ is especially effective as a pre-treatment $[9,22,28]$. Although results from several studies using $\mathrm{MI}$ in the domain of musculoskeletal care are already available $[1,21,27,56]$, a recent systematic review of $\mathrm{MI}$ as used in musculoskeletal health indicated the need for well-designed, adequately powered RCTs [14]. This led us to develop an Ml-based pre-treatment for patients starting pain rehabilitation.

Previous research on pain rehabilitation treatments has demonstrated the predictive value [49] and moderating role [20] of expectancy on treatment outcome, but further verification of this moderating role in particular is needed. In this study, the moderating role of treatment expectancy as well as the moderating role of the type of pain problem and motivation on treatment outcome were explored.

The objective of this study was to evaluate the effect of an MI-based pre-treatment compared to usual care pain education on the level of social participation, pain-related disability, and non-completion rates in patients with chronic non-specific musculoskeletal pain undergoing pain rehabilitation treatment. In addition, the influence of potentially moderating factors (expectancy, type of pain problem, and internal motivation orientation) on treatment effect were tested. 


\section{Methods}

\section{Study design}

The Prepare study was a nurse-led parallel-group two-armed single-blinded randomized controlled trial. A detailed description of the design and methods of this study are published elsewhere [32]. Randomization was conducted using computer-generated block randomization, using block sizes of four and an allocation ratio of 1:1. The study assistants and nurses involved were not blinded for treatment allocation. The trial was approved by the Medical Ethical Committee of the Maastricht University Medical Center and Maastricht University. The trial is registered in a public trials registry (Netherlands Trial Register, NTR3065). Study results have been reported according to the Consolidated Standards of Reporting Trials (CONSORT) [45]).

\section{Participants and intervention setting}

Between January 2012 and June 2013, study participants ( $n=163$ ) with non-specific chronic musculoskeletal pain indicated for pain rehabilitation treatment by a rehabilitation medicine consultant were recruited from two study sites: the outpatient rehabilitation medicine department of a university hospital and a regional hospital in the southern region of the Netherlands. Participants were randomly assigned to two nurse-led sessions of either MI-based pre-treatment (MIP) or pain education pre-treatment (UC). The participants received reimbursement of travel expenses for the pre-treatment they attended. After the pre-treatment, participants underwent a multidisciplinary screening to map the most suitable behaviorally-oriented pain rehabilitation approach (e.g. graded exposure, graded activity, Acceptance-Commitment Therapy (ACT)). The best treatment approach for each patient was then determined by a multidisciplinary team and patients entered a pain rehabilitation treatment lasting approximately 12 weeks.

\section{Inclusion/exclusion criteria}

Inclusion criteria were: non-specific chronic (duration $>3$ months) musculoskeletal pain (e.g. widespread pain (fibromyalgia), localized pain such as low back pain); age between 18 and 65 years; the chronic pain syndrome was not attributable to a recognizable, known specific pathology (for example infection, tumor, osteoporosis, fracture, structural deformity, inflammatory disorder such as ankylosing spondylitis); medium to high level of motivation for pain rehabilitation in the opinion of the rehabilitation medicine consultant; adequate literacy to complete assessment measures. Exclusion criteria were: pregnancy; surgery planned in the foreseeable future; involvement in litigation procedures; a psychiatric disease that might interfere with rehabilitation treatment (in the expert opinion of the rehabilitation medicine consultant).

\section{Nurses}

Four registered nurses were involved; two delivered MIP and two UC. In the MIP arm, the nurses received evidence-based training tailored to their specific needs and skills $[26,31,33,34,50]$. Furthermore, three subsequent training sessions took place during the intervention period. All training was provided by a certified $\mathrm{MI}$ trainer. The two nurses providing UC received training 
consisting of two parts: a refresher on general communication skills and the basic principles of health education, and discussion of relevant chapters from the book Mastering pain [58]. This training was provided by two of the authors (VCM and JV). During the intervention period, two follow-up sessions took place to discuss actual problems encountered during the delivery phase.

\section{Intervention description}

A detailed description of MIP and UC can be found in a related publication [32]. We opted to compare an education-based attention control group (UC) with an MI-based intervention group (MIP) to control for attention, as this is an important factor in provider-patient interactions $[7,11,12]$.

\section{Sample size}

The sample size calculation, which was carried out upon the difference in participation level (USER-P subscale satisfaction) at six-month follow-up compared to baseline as primary outcome, showed that a total of $n=128$ patients ( $n=64$ in each group) was needed to detect a mean difference of five points ( $S D=10)$ with $80 \%$ power, assuming an independent-samples t-test and a type I error rate $\alpha$ of 0.05 (two-sided). To allow for possible dropouts (20\%), the sample size of $n=160$ ( $n=80$ per arm) was chosen (for more information see [32]).

\section{Demographic and medical variables}

Baseline assessment included: gender, pain duration, type of pain problem (e.g. localized pain problem such as low back pain or widespread pain (fibromyalgia)), and pain intensity on a Visual Analogue Scale (VAS) [10].

\section{Outcome measures}

All patients filled out questionnaires sent by email or mail (upon request). The two research assistants responsible for collecting the data were not blinded to treatment allocation, but all questionnaires were administered electronically through a computer program.

\section{Primary outcome}

The primary outcome was the change in social participants' levels of participation six months after completing rehabilitation treatment compared to baseline [32]. Participation was measured using the Utrecht Scale for Evaluation of Rehabilitation-Participation (USER-P) [40]. This 32-item self-report measure covers three aspects of participation; namely: Frequency (11 items), Restriction (11 items), and Satisfaction (10 items). The totalled score of each four or five-point Likert subscale is calculated and then converted into a score ranging between 0 and 100 . Higher scores on the Likert scale reflect more social participation (higher frequency, less restrictions, higher satisfaction). No overall score could be calculated as these three subscales measure independent aspects of participation [40]. The psychometric qualities were satisfactory [40,53,55], though the level of responsiveness appeared to be lower in patients with chronic pain compared to patients with other diagnoses [53]. 


\section{Secondary outcomes}

Pain-related disability in performing daily activities resulting from chronic pain was measured using the Pain Disability Index (PDI) [39], which consists of seven items scored on an 11-point Likert scale ranging from 0 (no disability) to 10 (total disability). A total score is derived by adding up the item responses to give a sum ranging from 0 up to 70, with a higher score indicating more disability [39]. Good psychometric properties of the PDI have been demonstrated and normative data are available $[13,51]$. Non-completion of the rehabilitation treatment was registered in the patient data file at the institution. Non-completion could occur at three stages during the rehabilitation treatment, where patients were classified as either (1) not having started the screening, which was the initial part of the pain rehabilitation treatment when the exact treatment module was defined, (2) not having started the rehabilitation due to a negative recommendation from the treatment team after the screening, or (3) having dropped out of treatment prematurely.

\section{Other measures}

General self-efficacy was assessed using the Dutch General Self-efficacy questionnaire (GSE) [46]. Ten items ranging from "completely untrue" up to "completely true" assess ability to cope with general life demands. Higher scores indicate a more favorable outcome of more self-efficacy. A confirmatory factor analysis among elderly respondents [6] has previously shown a good fit. The level of depression was assessed using the Becks Depression Inventory (BDI)[4]. The BDI is a well-known instrument and is suitable for pain research. It is reliable, valid, and widely used $[2,3]$. Higher scores indicate a higher level of depressive symptoms. Both measures were employed to describe baseline characteristics of the trial population.

\section{Potential moderating factors}

1. Treatment credibility and expectancy was measured using an adapted and translated version of the Credibility and Expectancy Questionnaire (CEO) [15].

Participants were asked to rate a total of five items related to the credibility of and their expectancy regarding pain rehabilitation treatment. Two items were used to rate credibility and three items to rate expected success in terms of improvement in participation, decrease in pain-related disability, and decrease in pain intensity. The CEO uses a scale ranging from 1 (not at all) to 9 (very much) (total score range of 12-40 per subscale for credibility and expectancy). Higher scores indicate a more favorable higher credibility and expectancy. Good psychometric properties have previously been demonstrated and a two-factor structure confirmed [15].

2. Motivation was assessed using the Treatment Motivation Questionnaire (TMO). The TMO assesses intrinsic and extrinsic motivation for entering and remaining in treatment [43]. It consists of 26 items that are rated on a seven-point Likert scale ranging from 1 (not at all true) up to 7 (very true). The factors of internal and external motivation, interpersonal help-seeking, and confidence in treatment were taken into account. Higher scores indicate higher internal motivation, higher external motivation, higher interpersonal help-seeking, and higher confidence in 
treatment. Items were slightly adapted to the rehabilitation context. The TM0 correlates well with professionals' ratings of the above-mentioned factors, suggesting good construct validity [43].

3. Type of pain problem (i.e. localized pain problem (e.g. complex regional pain syndrome (CRPS), pain in arm or leg, chronic low back pain) vs. widespread pain (fibromyalgia)) was assessed during the recruitment phase by means of the patient data file.

\section{Statistical analysis}

To test for differences in numerical and categorical variables between the groups at baseline, independent-samples t-tests or Mann-Whitney U tests, as appropriate, and Chi square tests were used, respectively. The effects of the intervention were investigated using the intention-to-treat (ITT) approach, which included all participants as randomised. The primary analysis of intervention effect on the numeric outcome variables used linear mixed model analyses, with group, time (categorical), and group*time as fixed factors, to test differences in outcome at each time-point. Different options were considered for the random part of the model (random intercept and/or slope, or no random effects with unstructured covariance structure for repeated measures), with that ultimately chosen based on Akaike's Information Criterion (AIC). To check whether missing outcome data depended on baseline variables, logistic regression analyses were executed with missing (yes/no) as outcome and one of the baseline variables as explanatory variable. No imputation of missing data took place, since the likelihood-based approach was used for missing outcomes, assuming missing at random (MAR). As a sensitivity analysis, the same model was applied to the data, with imputation of the groups' mean per outcome, per measurement moment in the case of a missing value. In addition, a per-protocol ("on treatment") approach was used in which only participants who entered and completed the pre-treatment and subsequent pain rehabilitation treatment were included. The same sensitivity analysis for missing data was carried out here as well. A Chi-square test (i.e. treatment completed yes/no), was used for the primary analysis of intervention effect on the categorical outcome variable. Finally, moderation analyses were carried out for each outcome variable, which took separate account of expectancy, nature of pain (localized pain vs. widespread pain (fibromyalgia)), and motivation orientation.

The data analysts (VCM and BW) were blinded during the baseline comparisons, intention-to-treat analyses, and per-protocol analyses. For the moderation analyses, blinding (thus patient allocation) was broken. Data were analysed using IBM SPSS Statistics for Windows version 22 (IBM Corp., Armonk, NY, USA). A two-sided P value $<0.05$ was considered to be statistically significant in all analyses. 


\section{Results}

\section{Flow of participants}

Figure 1 shows a CONSORT flow diagram of participants' progression through the study period including withdrawals and reasons at each stage of measurement.

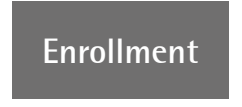

Assessed for eligibility $(n=213)$

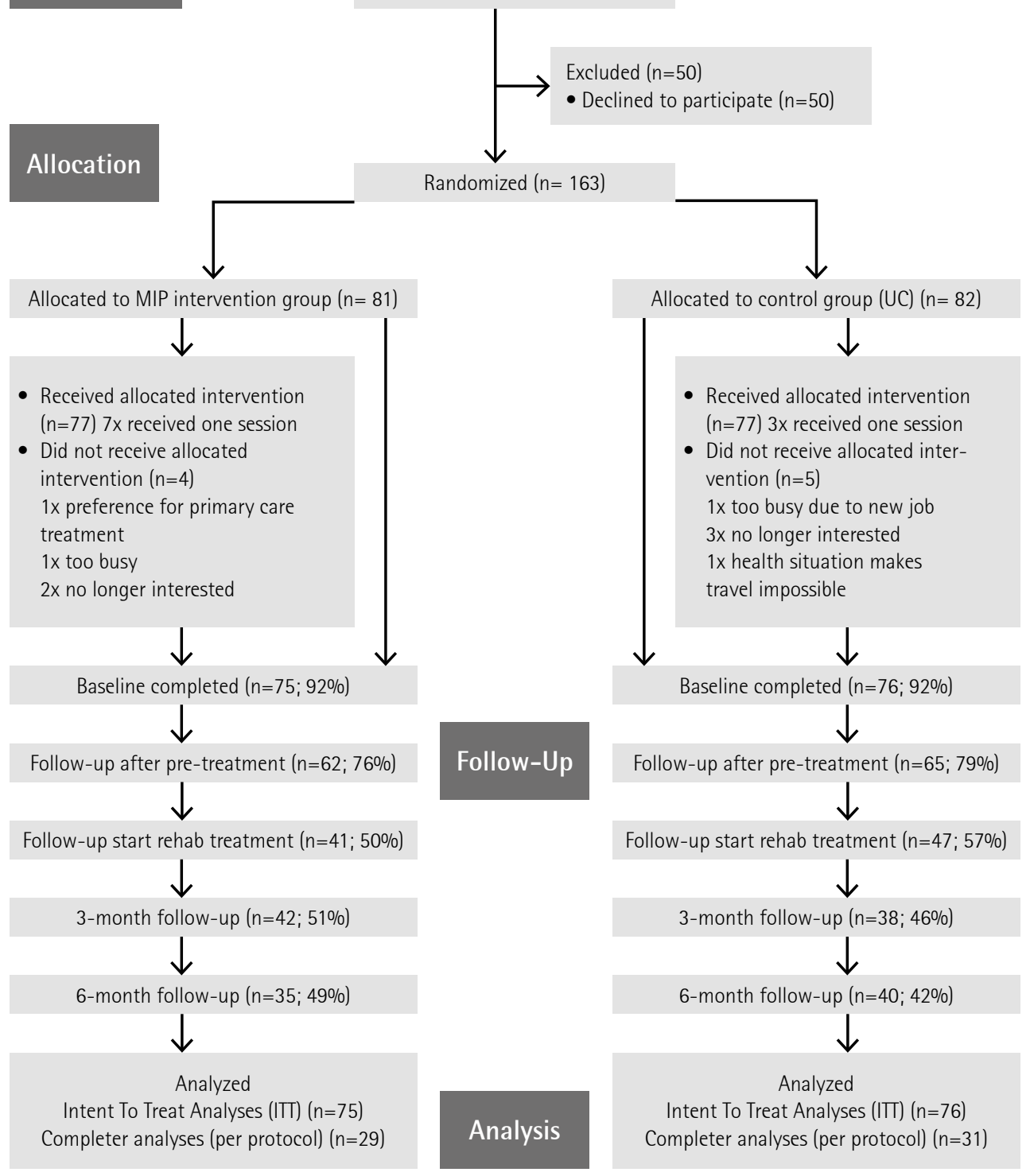

Figure 1 Flow of participants through Prepare trial 
The RCT took place between January 2012, when the first participant was included in the study, and September 2014, when the final follow-up measurement was completed. In total, 163 participants were included in the study. Randomization allocated 81 participants to the intervention condition (MIP) and 82 to the education-based control condition (UC). Twelve patients ( $n=6$ MIP; $n=6$ UC) did not complete the baseline measurement after randomization. The reasons for non-completion are presented in figure 1. Chi-square tests revealed no significant differences between patients who completed the two pre-treatment sessions of either MIP (90.7\%) or UC $(92.1 \%)(p=.753)$.

As can be deduced from table 1 (see next page), the MIP and UC conditions at baseline did differ significantly in terms of ages $(p<.001)$. The groups did not differ in other socio-demographic characteristics, level of participation, pain-related disability, or other secondary outcomes at the time of inclusion (all $p>$.20).

\section{Treatment outcome: intention-to-treat analyses}

No significant difference was found between MIP and UC as regards participation frequency ( $p=.804)$, restrictions $(p=.298)$, or satisfaction $(p=.736)$, or in the secondary outcome of painrelated disability $(p=.087)$ six months after treatment. Standardized effect sizes (absolute Cohen's $d,|d|)$ were generally small $(|d|<.20)$. Moderate standardized effect sizes $(|d|<0.32)$ were found for both participation frequency and participation. Sensitivity analyses, using mean imputation, gave similar results for restrictions after treatment, and for pain-related disability at the six-month follow-up. See table 2 for an overview.

Non-completion of the pain rehabilitation treatment was 30\% $(n=23)$ in MIP and 35\% $(n=27)$ in UC, which is not significantly different $(p=.526)$ (also see table 1 ). As already mentioned, non-completion could occur at three different stages during the rehabilitation treatment; namely: (1) 7 participants ( $n=3$ MIP; $n=4$ UC) did not start the screening, (2) 14 participants ( $n=6$ MIP; $n=8$ UC) were found to be unsuitable for pain rehabilitation treatment after screening by the treatment team and 18 participants decided on their own not to start the pain rehabilitation treatment after the screening ( $n=9$ MIP; $n=9$ UC), and (3) 11 ( $n=5$ MIP; $n=6$ UC) stopped the pain rehabilitation treatment prematurely. No differences between the two treatment conditions were found. Logistic regression taking into account gender, age, pain duration, and nurse showed that participants who did not complete the pain rehabilitation treatment did not differ significantly in terms of baseline variables from those who did finish (all $p$-values $>.25$ ).

\section{Treatment outcome: per-protocol analyses}

Analyses including only the patients who completed pre-treatment and rehabilitation treatment corroborated the results of the intention-to-treat analyses for all primary outcomes of the USER-P. At the six-month follow-up measurement, participants in the MIP condition showed a larger decrease in pain-related disability (PDI) (indicating an increase in the UC condition), pointing to a significant difference between the groups (treatment effect $(95 \% \mathrm{Cl})=7.52(1.07-13.98)$, $p=.023)$. 
Table 1: Baseline characteristics and baseline outcome measures of the total sample and for each treatment group

\begin{tabular}{|c|c|c|c|c|c|c|c|c|}
\hline & Abbr. & \multicolumn{2}{|c|}{$\begin{array}{c}\text { Total } \\
(\mathrm{N}=151)^{*}\end{array}$} & \multicolumn{2}{|c|}{$\begin{array}{l}\text { MIP intervention } \\
\qquad(\mathrm{N}=75)^{*}\end{array}$} & \multicolumn{2}{|c|}{$\begin{array}{l}\text { UC control } \\
\qquad(N=76)^{*}\end{array}$} & $\mathrm{p}$ \\
\hline \multicolumn{9}{|l|}{ Demographic variables } \\
\hline Age (y) & & 46.59 & $( \pm 11.59)$ & 47.83 & $( \pm 11.17)$ & 45.41 & $( \pm 11.94)$ & .001 \\
\hline Gender ( $\%$ female $)^{a}$ & & $71.5 \%$ & & $69.3 \%$ & & $69.7 \%$ & & .897 \\
\hline $\begin{array}{l}\text { Duration of chronic pain } \\
\text { complaints }(y)^{\wedge}\end{array}$ & & 6.00 & $\begin{array}{l}(\mathrm{IQR}) \\
2-14\end{array}$ & 5.00 & $\begin{array}{l}(\mathrm{IQR}) \\
2-12\end{array}$ & 8.50 & $\begin{array}{c}(\mathrm{IOR}) \\
3-15.25\end{array}$ & .269 \\
\hline $\begin{array}{l}\text { Diagnosis: } \\
\text { Fibromyalgia }^{a}\end{array}$ & & $34.5 \%$ & & $31.6 \%$ & & $37.5 \%$ & & .567 \\
\hline $\begin{array}{l}\text { Pain intensity: } \\
\text { At this moment }\end{array}$ & VAS $1-10$ & 6.32 & $( \pm 1.98)$ & 6.08 & $( \pm 2.04)$ & 6.56 & $( \pm 1.91)$ & .226 \\
\hline \multicolumn{9}{|c|}{ Primary outcome effect evaluation } \\
\hline $\begin{array}{l}\text { Social participation: } \\
\text { Restrictions }\end{array}$ & USER-P & 68.70 & $( \pm 15.20)$ & 67.21 & $( \pm 15.45)$ & 70.13 & $( \pm 14.91)$ & .245 \\
\hline $\begin{array}{l}\text { Social participation: } \\
\text { Satisfaction }\end{array}$ & USER-P & 51.44 & $( \pm 17.82)$ & 51.14 & $( \pm 17.12)$ & 51.73 & $( \pm 18.56)$ & .846 \\
\hline Social participation: Frequency & USER-P & 30.65 & $( \pm 10.70)$ & 29.69 & $( \pm 11.33)$ & 31.58 & $( \pm 10.04)$ & .285 \\
\hline \multicolumn{9}{|c|}{ Secondary outcomes effect evaluation } \\
\hline Pain-related disability & PDI & 39.76 & $( \pm 11.49)$ & 41.17 & $( \pm 10.90)$ & 38.40 & $( \pm 11.95)$ & .202 \\
\hline Pre-treatment completed ${ }^{a}$ & & $91.4 \%$ & & $90.7 \%$ & & $92.1 \%$ & & .753 \\
\hline $\begin{array}{l}\text { Non-completion } \\
\text { rehabilitation treatment }{ }^{a}\end{array}$ & & $33.1 \%$ & & $30.7 \%$ & & $35.5 \%$ & & .526 \\
\hline \multicolumn{9}{|l|}{ Other measures } \\
\hline Self-efficacy $(10-40)^{\wedge}$ & GSE & 29.00 & $\begin{array}{c}(\mathrm{IQR}) \\
25-34\end{array}$ & 28.50 & $\begin{array}{c}(\mathrm{IQR}) \\
25-33.25\end{array}$ & 30.00 & $\begin{array}{c}(\mathrm{IOR}) \\
24-34\end{array}$ & .609 \\
\hline Depressive symptoms $(0-63)^{\wedge}$ & $\mathrm{BDI}$ & 13.00 & $\begin{array}{c}\text { (IQR) } \\
10.5-20.5\end{array}$ & 13.00 & $\begin{array}{c}(\text { IOR) } \\
9.45-20\end{array}$ & 13.00 & $\begin{array}{c}\text { (IQR) } \\
10.91-14\end{array}$ & .605 \\
\hline \multicolumn{9}{|l|}{ Potential moderating factors } \\
\hline Treatment credibility ${ }^{\wedge}$ & CEQ & 19.33 & $\begin{array}{c}\text { (IQR) } \\
16.66-22.33\end{array}$ & 19.00 & $\begin{array}{c}(\text { IQR }) \\
15.66-21.33\end{array}$ & 19.83 & $\begin{array}{c}\text { (IQR) } \\
17-22.66\end{array}$ & .311 \\
\hline Treatment expectancy & CEQ & 13.16 & $\begin{array}{c}(\mathrm{IQR}) \\
11-14.33\end{array}$ & 13.33 & $\begin{array}{c}(\mathrm{IQR}) \\
11.41-14.66\end{array}$ & 13.00 & $\begin{array}{c}(\mathrm{IQR}) \\
10.91-14\end{array}$ & .212 \\
\hline External motivation & TMQ & 3.40 & $( \pm 1.19)$ & 3.51 & $( \pm 1.22)$ & 3.29 & $( \pm 1.16)$ & .261 \\
\hline Internal motivation & TMQ & 5.27 & $\begin{array}{l}(I Q R) \\
4.63-6\end{array}$ & 5.27 & $\begin{array}{c}(\mathrm{IQR}) \\
4.52-5.9\end{array}$ & 5.40 & $\begin{array}{c}(I Q R) \\
4.70-6.02)\end{array}$ & .763 \\
\hline Help-seeking motivation & TM0 & 4.73 & $( \pm 1.48)$ & 4.64 & $( \pm 1.52)$ & 4.82 & $( \pm 1.44)$ & .448 \\
\hline Confidence in treatment & TMO & 3.71 & $( \pm 0.93)$ & 3.76 & $( \pm 0.94)$ & 3.66 & $( \pm 0.92)$ & .495 \\
\hline
\end{tabular}

* Information based upon $n=151$ who filled in the baseline questionnaire ( $n=163$ total group, $n=82$ randomized to MIP intervention group and $n=81$ randomized to UC control group)

$a_{x} 2$ tests, independent t-tests or Mann-Whitney $U$ tests for comparisons between treatment groups with $\mathrm{a}=.005$.

Values presented are means and standard deviations $( \pm S D)$, or percentages, except when marked with ${ }^{\prime}$, when medians and interquartile ranges (IQR) are presented since skewness exceeded -1 or 1 . 
Table 2: Observed means, ( \pm standard deviations (SD), standardized effect sizes (Cohen's $d$ ), and estimated mean differences (95\% Cl, p-value)) based on linear mixed models (corrected for baseline) for the primary and secondary outcome measures
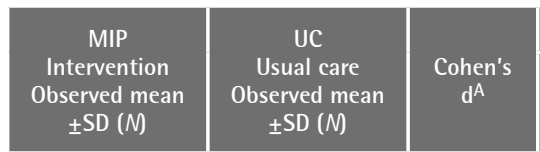

\begin{tabular}{|c|c|c|c|}
\hline \multicolumn{4}{|c|}{ Linear mixed models } \\
\hline $\begin{array}{c}\text { Estimated } \\
\text { mean } \\
\text { difference }\end{array}$ & $\begin{array}{c}\text { Lower } \\
95 \% \mathrm{Cl}\end{array}$ & $\begin{array}{c}\text { Upper } \\
95 \% \mathrm{Cl}\end{array}$ & p-value \\
\hline
\end{tabular}

Primary outcomes

\section{Social participation (USER-P), Frequency (0-100)}

\begin{tabular}{|c|c|c|c|c|c|c|c|}
\hline Baseline to & $\begin{array}{c}29.69 \pm 11.33 \\
(N=72)\end{array}$ & $\begin{array}{c}31.58 \pm 10.04 \\
(N=75)\end{array}$ & & & & & \\
\hline $\begin{array}{l}\text { After pre-treat- } \\
\text { ment t1 }\end{array}$ & $\begin{array}{c}31.12 \pm 10.91 \\
(N=62)\end{array}$ & $\begin{array}{c}31.06 \pm 10.87 \\
(N=65)\end{array}$ & 0.05 & -.98 & -3.65 & 1.69 & .468 \\
\hline Post-treatment $\mathrm{t} 3$ & $\begin{array}{c}31.25 \pm 13.09 \\
(N=42)\end{array}$ & $\begin{array}{c}34.98 \pm 11.98 \\
(N=37)\end{array}$ & -0.29 & .95 & -2.96 & 4.87 & .629 \\
\hline $\begin{array}{l}\text { Six months fol- } \\
\text { low-up t4 }\end{array}$ & $\begin{array}{c}32.15 \pm 11.22 \\
(\mathrm{~N}=35)\end{array}$ & $\begin{array}{c}32.11 \pm 13.09 \\
(N=40)\end{array}$ & 0.03 & .50 & -3.54 & 4.55 & .804 \\
\hline \multicolumn{8}{|c|}{ Restrictions $(0-100)$} \\
\hline Baseline t0 & $\begin{array}{c}67.21 \pm 15.45 \\
(N=72)\end{array}$ & $\begin{array}{c}70.13 \pm 14.91 \\
(N=75)\end{array}$ & & & & & \\
\hline $\begin{array}{l}\text { After pre-treat- } \\
\text { ment } t 1\end{array}$ & $\begin{array}{c}66.15 \pm 14.36 \\
(N=62)\end{array}$ & $\begin{array}{c}71.19 \pm 14.87 \\
(N=65)\end{array}$ & -0.19 & 1.94 & -2.62 & 6.49 & .403 \\
\hline Post-treatment t 3 & $\begin{array}{c}75.51 \pm 16.76 \\
(N=42)\end{array}$ & $\begin{array}{c}80.62 \pm 14.84 \\
(N=37)\end{array}$ & -0.32 & -.96 & -7.24 & 5.32 & .764 \\
\hline $\begin{array}{l}\text { Six months fol- } \\
\text { low-up t4 }\end{array}$ & $\begin{array}{c}79.84 \pm 18.49 \\
(N=35)\end{array}$ & $\begin{array}{c}77.05 \pm 16.58 \\
(N=40)\end{array}$ & 0.15 & -3.80 & -11.00 & 3.39 & .298 \\
\hline \multicolumn{8}{|c|}{ Satisfaction (0-100) } \\
\hline Baseline t0 & $\begin{array}{c}51.14 \pm 17.12 \\
(N=67)\end{array}$ & $\begin{array}{c}51.73 \pm 18.56 \\
(N=75)\end{array}$ & & & & & \\
\hline $\begin{array}{l}\text { After pre-treat- } \\
\text { ment } t 1\end{array}$ & $\begin{array}{c}49.46 \pm 15.93 \\
(N=57)\end{array}$ & $\begin{array}{c}50.03 \pm 18.47 \\
(N=63)\end{array}$ & -0.03 & -.12 & -5.20 & 4.95 & .962 \\
\hline Post-treatment $\mathrm{t} 3$ & $\begin{array}{c}58.03 \pm 20.16 \\
(N=40)\end{array}$ & $\begin{array}{c}65.80 \pm 20.47 \\
(N=36)\end{array}$ & 0.04 & 7.15 & -.13 & 14.43 & .054 \\
\hline $\begin{array}{l}\text { Six months } \\
\text { follow-up t4 }\end{array}$ & $\begin{array}{c}62.87 \pm 19.93 \\
(N=35)\end{array}$ & $\begin{array}{c}59.33 \pm 22.47 \\
(N=39)\end{array}$ & 0.16 & -1.40 & -9.64 & 6.83 & .736 \\
\hline
\end{tabular}

Secondary outcomes

Pain-related disability, PDI (0-70)B

\begin{tabular}{|c|c|c|c|c|c|c|c|}
\hline Baseline t0 & $\begin{array}{c}41.17 \pm 10.90 \\
(N=55)\end{array}$ & $\begin{array}{c}38.40 \pm 11.96 \\
(N=57)\end{array}$ & & & & & \\
\hline Start treatment t2 & $\begin{array}{c}36.67 \pm 12.87 \\
(N=41)\end{array}$ & $\begin{array}{c}34.65 \pm 11.05 \\
(\mathrm{~N}=47)\end{array}$ & 0.16 & 1.43 & -3.78 & 6.63 & .587 \\
\hline Post-treatment $\mathrm{t} 3$ & $\begin{array}{c}31.62 \pm 15.01 \\
(\mathrm{~N}=42)\end{array}$ & $\begin{array}{c}30.39 \pm 16.20 \\
(N=38)\end{array}$ & 0.07 & 3.86 & -1.56 & 9.28 & .161 \\
\hline $\begin{array}{l}\text { Six months fol- } \\
\text { low-up t4 }\end{array}$ & $\begin{array}{c}28.36 \pm 16.67 \\
(\mathrm{~N}=35)\end{array}$ & $\begin{array}{c}32.95 \pm 13.57 \\
(\mathrm{~N}=40)\end{array}$ & -0.30 & 4.97 & -.73 & 10.66 & .087 \\
\hline
\end{tabular}

${ }^{A}$ Cohen's $d$ is calculated as follows: The means of the particular follow-up moment for the two conditions divided by the pooled standard deviation, ${ }^{\mathrm{B}} \mathrm{Higher}$ values on the PDI are referring to a higher level of pain-related disability 


\section{Moderation analyses}

Type of pain problem (localized pain vs. fibromyalgia), expectancy, and motivation orientation were taken into account in the moderation analyses. Results of the moderation analyses showed that type of pain problem had a significant moderating effect (see table 3).

Table 3: Estimated mean differences between groups at six-month follow-up, corrected for baseline and for type of pain problem (localized pain vs fibromyalgia) with corresponding $95 \% \mathrm{Cl}$ and $p$-values as well as overall $p$-values of interaction terms

\begin{tabular}{|l|l|c|c|c|c|}
\multicolumn{2}{|c|}{$\begin{array}{c}\text { Moderation } \\
\text { analyses diagnosis }\end{array}$} & $\begin{array}{c}\text { Estimated } \\
\text { mean dif- } \\
\text { ferences } \\
\text { UC-MIP }\end{array}$ & $\begin{array}{c}\text { Lower } \\
95 \% \mathrm{Cl}\end{array}$ & $\begin{array}{c}\text { Upper } \\
95 \% \mathrm{Cl}\end{array}$ & p-value \\
\hline USER-P Restrictions & & & & 0.023 \\
\hline $\begin{array}{l}\text { Six months } \\
\text { follow-up }\end{array}$ & fibromyalgia & 7.27 & -4.70 & 19.25 & 0.231 \\
\hline Pain-related disability (PDI) & -9.69 & -17.82 & -1.57 & 0.020 \\
\hline $\begin{array}{l}\text { Six months } \\
\text { follow-up }\end{array}$ & fibromyalgia & -2.07 & -11.75 & 7.60 & 0.074 \\
\hline
\end{tabular}

The estimated mean difference (UC-MIP) for participation restrictions at six-month follow-up, corrected for baseline, depended significantly on the type of pain problem ( $p=.023)$. Within the group with a localized pain problem, there was a significant difference (-9.69 points, $p=.020$ ), favoring MIP, whereas for the group with fibromyalgia, there was a non-significant difference of 7.27 points ( $p=.231)$, favoring UC. For pain-related disability, no significant differences between pre-treatment conditions within the group of patients with fibromyalgia could be found $(-2.07$ points, $p=671$ ), but pain-related disability in patients with a localized pain problem showed a significant and clinically relevant decrease (8.68 points, $p=.012$ ). For all other variables, clearly non-significant and non-clinically relevant differences were found. 


\section{Discussion}

To our knowledge, this is the first RCT to assess the effect of MI-based pre-treatment (MIP) prior to chronic pain rehabilitation treatment. The results indicate that the MIP condition was not more effective compared to nurse-led pain education pre-treatment (UC) in terms of social participation or pain-related disability, though the group difference for pain-related disability almost reached significance favoring MIP. Non-completion of the rehabilitation treatment did not differ significantly between types of pre-treatment. Taking only those patients into account who after the pre-treatment actually started and finished the rehabilitation treatment, the MIP condition showed a significantly higher improvement in pain-related disability, but not in social participation. Moderation analyses showed that MIP was more effective in one specific subgroup: patients with a localized pain problem showed a significant improvement in participation and a clinically relevant reduction of pain-related disability.

In line with findings of modest to moderate effectiveness of behaviorally-oriented rehabilitation treatments (overall chronic pain in low back pain and fibromyalgia) in other systematic reviews $[19,23,57]$, our rehabilitation treatment was effective overall, but no added effect attributable to $\mathrm{Ml}$ could be demonstrated. This is contrary to comparable research on the use of $\mathrm{Ml}$ in the care of chronic pain patients, which concluded that MI-based approaches were effective. Vong et al. (2011) evaluated a slightly adapted version of MI, namely Motivational Enhancement Therapy (MET), versus conventional physical therapy in patients with chronic low back pain in an ambulant setting [56]. MET produced significant improvements in physical functioning and exercise compliance, as well as trends in reduction of pain intensity and functional disability. A contrast with our study intervention is that MET was not provided as pre-treatment and only one discipline (physical therapist) was involved. Embedment of MET within the ongoing treatment may have be attributable for this difference. However, systematic reviews do attest that MI can effectively serve as a stand-alone pre-treatment $[9,22,28]$. A drawback of the Vong et al. study is that the content of MET and of MET training was not clearly described and no validated measure of fidelity was used.

Habib et al. (2005) evaluated the effectiveness of two Ml-based pre-treatment sessions for patients with chronic pain planning to undergo a rehabilitation treatment [21]. The amount and the timing of pre-treatment were similar to our MIP condition, but those providing it were psychologists instead of nurses. This could mean that psychologists, who have more training in general counseling skills, are more able than nurses to deliver proper MI. Another difference with our trial was that attendance at subsequent pain management workshops was considered as endpoint; 
patients in the MI treatment group were significantly more likely to attend the workshops.

There are at least four factors that may explain our findings: (1) the adequate indication of patients for chronic pain rehabilitation treatment, (2) fidelity and quality issues, (3) the choice of participation as primary outcome measure, and (4) the effectiveness of MI in different patient groups. Each of these aspects is discussed separately below.

(1) In this trial, the number of patients who were indicated for rehabilitation treatment by the rehabilitation medicine consultant but who did not pass the multidisciplinary screening afterwards or decided on their own not to start or continue the rehabilitation treatment was high. The rate of non-completion of the behaviorally-oriented rehabilitation treatment (30\%) was comparable with dropout rates in CBT treatments for patients with chronic low back pain (between 13-30\%) [18]. Ultimately, it will have to be determined which approach is better: whether to set strict criteria for the selection of patients for pain rehabilitation at an initial stage or to take a more laissez-faire approach until the multidisciplinary screening has provided a final indication.

(2) In behavioral interventions such as MI, verification of quality and fidelity is important but often lacking [36]. The process evaluation of this trial, which was carried out before the effectiveness evaluation, demonstrated that the fidelity and quality of this MI-based intervention (MIP) condition was not satisfactory for all aspects of MI (Mertens et al., 2015), even though the nurses received extensive and targeted training. The findings regarding effectiveness should therefore be taken with caution. In line with other studies, we can conclude that MI training requires a huge investment of time and resources. Moreover, it is very likely that not everyone can reach a sufficient level of MI proficiency (e.g. [16] independent of the type of provider, as a previous systematic review has shown) [29]. It has been demonstrated that although different types of providers can learn MI, MI training requires considerable effort.

(3) In the field of rehabilitation, social participation is the most relevant outcome of all efforts during treatment. We therefore chose participation as primary outcome and measured it using the USER-P. The advantage of the USER-P is that it provides a generic measurement of the frequency and restrictions of, as well as satisfaction with participation. At the time that we selected our primary outcome measure, none of the available instruments had been sufficiently tested for their clinimetric properties. It was only during the trial that more information on the psychometric properties of the USER-P instrument became available. The most important concern is the instrument's responsiveness. Studies show that the effect size in patients with chronic pain is rather low in comparison with other diagnostic groups such as brain injury or heart conditions (e.g. USER-P frequency for chronic pain is 0.07 , compared to 0.70 for brain injury and 0.58 for heart conditions) [53], which may be explained by the fact that large score differences are required for change [55]. This could mean that the USER-P is less suitable as outcome measure for the effectiveness of pain rehabilitation treatments as its content is too generic and therefore its sensitivity to change (and ability to detect it) too low. Another concern in measuring participa- 
tion in patients with chronic pain is the fluctuating nature of chronic pain; this could influence participation but is not accounted for in the USER-P. Notwithstanding these concerns, however, when compared with other participation assessment instruments (such as IPA, WHODAS, and IMPACT), the psychometric quality of the USER-P is still better [54]. Therefore, the USER-P is the best available measure of the three different aspects of participation. By contrast, the Pain Disability Index (PDI), used to measure disability, was developed specifically for patients with chronic pain and information on what constitutes meaningful clinical change is available [51]. Taking the aforementioned concerns into account, we have chosen to present and discuss all findings of the PDI in this paper.

(4) The moderation analyses showed that patient-specific characteristics indeed influenced the effectiveness of MI. Patients with a more localized pain syndrome improved in perceived participation restrictions and pain-related disability over time, but patients diagnosed with fibromyalgia did not. All patients received a behaviorally-oriented treatment. The emphasis of the treatment for patients with fibromyalgia was oriented towards an Acceptance Commitment Therapy (ACT) approach, a so-called third-wave cognitive-behavioral therapy, compared to more second-wave cognitive-behavioral type rehabilitation treatments (graded activity, graded exposure) for patients with more localized pain syndromes. It is possible that the treatments offered to the different pain populations are less comparable than we had assumed. Another explanation could be that MI may not be suitable at all for patients with fibromyalgia, but only for patients with localized pain syndromes. Our results indicate that the question of what works for whom still needs to be further explored.

A potential limitation of this evaluation is that per-protocol analysis may have introduced bias. Due to patients dropping out during pre-treatment and treatment, the randomly allocated intervention and control group may no longer be comparable. Even though we did check for selective dropout and concluded that there were no variables significantly related to dropout, in the end complete comparability cannot be guaranteed. Furthermore, high rates of non-completion of rehabilitation treatment do challenge the validity of our trial. And despite the fact that our study was not powered for moderation analyses, a significant moderation effect was found. 


\section{Conclusion}

In conclusion, this study revealed that a nurse-led MI-based pre-treatment (MIP) was not more effective compared to pain education pre-treatment (UC) in improving long-term social participation after rehabilitation treatment. Per-protocol analysis taking into account only those patients who completed their pre-treatment and rehabilitation treatment showed a larger decrease in related disability, which resulted in a statistically significant difference between both groups. Moderation analysis showed that MIP is effective for improving participation and decreasing pain-related disability in patients with a localized pain problem but not in patients with fibromyalgia. We have presented a broad outline of potential reasons for the current neutral findings and conclude that further research is needed to entangle specific moderating factors and the influence of the type of pain problem on the outcome of the treatment approach. More research into MI skills training in nurse-led care is also needed.

\section{Acknowledgements}

The authors wish to thank the patients and nurses who took part in the Prepare study for their participation, and Wilma Noteborn (of the Mondriaan clinic in Heerlen) for the education component of the MI-based pre-treatment. This PhD project was funded by Adelante zorggroep, Hoensbroek, the Netherlands.

\section{List of abbreviations}

\section{Abbreviation}

\begin{tabular}{|l|l|}
\hline MI & Motivational Interviewing \\
\hline MIP & Motivational Interviewing-based pre-treatment \\
\hline Prepare & Pre-Pain rehabilitation \\
\hline UC & Usual Care \\
\hline
\end{tabular}




\section{References}

[1] Ang DC, Kaleth AS, Bigatti S, Mazzuca SA, Jensen MP, Hilligoss J, Slaven J, Saha C. Research to encourage exercise for fibromyalgia (REEF): use of motivational interviewing, outcomes from a randomized-controlled trial. Clin J Pain 2013;29(4):296-304.

[2] Beck AT, Steer RA. Beck Depression Inventory. San Antonio: Psychological Corporation, 1993.

[3] Beck AT, Steer RA, Carbin MG., Psychometric properties of the Beck Depression Inventory: Twenty-five years of evaluation. Clin Psychol Rev 1988;8(1):77-100.

[4] Beck AT, Ward CH, Mendelsohn M, Mock J, Erbaugh J., An inventory for measuring depression. Arch Gen Psychiatry 1961;4:561-571.

[5] Bendix AE, Bendix T, Haestrup C, Busch E., A prospective, randomized 5-year follow-up study of functional restoration in chronic low back pain patients. Eur Spine J 1998;7(2):111-119.

[6] Bosscher RJ, Smit JH., Confirmatory factor analysis of the General Self-Efficacy Scale. Behav Res Ther 1998;36(3):339-343.

[7] Braunholtz DA, Edwards SJL, Lilford RJ., Are randomized clinical trials good for us (in the short term)? Evidence for a "trial effect". J Clin Epidemiol 2001;54(3):217-224.

[8] Breivik H, Collett B, Ventafridda V, Cohen R, Gallacher D., Survey of chronic pain in Europe: prevalence, impact on daily life, and treatment. Eur J Pain 2006;10(4):287-333.

[9] Burke BL, Arkowitz H, Menchola M., The efficacy of motivational interviewing: a metaanalysis of controlled clinical trials. J Consult Clin Psychol 2003;71(5):843-861.

[10] Carlsson AM. Assessment of chronic pain. I. Aspects of the reliability and validity of the visual analogue scale. Pain 1983;16(1):87-101.

[11] Cherkin DC, Deyo RA, Street JH, Hunt M, Barlow W., Pitfalls of patient education. Limited success of a program for back pain in primary care. Spine (Phila Pa 1976) 1996;21(3):345-355. 
[12] Cherkin DC, Eisenberg D, Sherman KJ, et al., Randomized trial comparing traditional chinese medical acupuncture, therapeutic massage, and self-care education for chronic low back pain. Arch Intern Med 2001;161(8):1081-1088.

[13] Chibnall JT, Tait RC., The Pain Disability Index: factor structure and normative data. Arch Phys Med Rehabil 1994;75(10):1082-1086.

[14] Chilton R, Pires Yfantouda R, Wylie M., A systematic review of motivational interviewing within musculoskeletal health. Psychol Health Med 2012.

[15] Devilly GJ, Borkovec TD., Psychometric properties of the credibility/expectancy question naire. J Behav Ther Exp Psychiatry 2000;31(2):73-86.

[16] Forsberg L, Forsberg LG, Lindqvist $H$, Helgason AR., Clinician acquisition and retention of Motivational Interviewing skills: a two-and-a-half-year exploratory study.

Subst Abuse Treat Prev Policy 2010;5:8.

[17] Gaskin DJ, Richard P., The economic costs of pain in the United States. J Pain 2012;13(8):715-724.

[18] Hartwich-Tersek J, Rief W., Attrition in Cognitive-behavioral Treatment of Chronic Back Pain. Clin J Pain 2010;26(7):593-601.

[19] Glombiewski JA, Sawyer AT, Gutermann J, Koenig K, Rief W, Hofmann SG., Psychological treatments for fibromyalgia: A meta-analysis. Pain 2010;151(2):280-295.

[20] Goossens ME, Vlaeyen JW, Hidding A, Kole-Snijders A, Evers SM., Treatment expectancy affects the outcome of cognitive-behavioral interventions in chronic pain.

Clin J Pain 2005;21(1):18-26; discussion 69-72.

[21] Habib S, Morrissey S, Helmes E., Preparing for pain management: a pilot study to enhance engagement. J Pain 2005;6(1):48-54.

[22] Hettema J, Steele J, Miller WR., Motivational interviewing. Annu Rev Clin Psychol 2005;1:91-111.

[23] Kamper SJ, Apeldoorn AT, Chiarotto A, Smeets RJ, Ostelo RW, Guzman J, Van Tulder MW., Multidisciplinary biopsychosocial rehabilitation for chronic low back pain. The Cochrane Database of Systematic Reviews 2014;9:CD000963. 
[24] Köke A, Brouwers M, Heuts P, Schiphorst Preuper R, Smeets R, Swaan L, Vlaeyen J, Patijn J. Consensus Rapport Pijnrevalidatie Nederland. Een beschrijvend overzicht van pijnrevalidatieprogramma's in Nederland met betrekking tot doelen en inhoud. [Consensus Report on pain rehabilitation in the Netherlands. A descriptive overview of pain rehabilitaion programs in the Netherlands as regards goals and content]. Maastricht: PijnKennis Centrum Maastricht [Pain Knowledge Centre Maastricht], 2005.

[25] Lambeek LC, Van Tulder MW, Swinkels IC, Koppes LL, Anema JR, Van Mechelen W., The trend in total cost of back pain in The Netherlands in the period 2002 to 2007. Spine 2011;36(13):1050-1058.

[26] Lane $C$, Hood K, Rollnick S., Teaching motivational interviewing: Using role play is as effective as using simulated patients. Med Educ 2008;42(6):637-644.

[27] Leonhardt C, Keller S, Chenot JF, Luckmann J, Basler HD, Wegscheider K, Baum E, Donner-Banzhoff N, Pfingsten M, Hildebrandt J, Kochen MM, Becker A., TM-based motivational counselling does not increase physical activity of low back pain patients in a primary care setting - A cluster-randomized controlled trial. Patient Educ Couns 2008;70(1):50-60.

[28] Lundahl B, Burke BL., The effectiveness and applicability of motivational interviewing: A practice-friendly review of four meta-analyses. J Clin Psychol 2009;65(11):1232-1245.

[29] Lundahl B, Moleni T, Burke BL, Butters R, Tollefson D, Butler C, Rollnick S., Motivational interviewing in medical care settings: a systematic review and meta-analysis of randomized controlled trials. Patient Educ Couns 2013;93(2):157-168.

[30] Lundahl BW, Kunz C, Brownell C, Tollefson D, Burke BL., A meta-analysis of motivational interviewing: Twenty-five years of empirical studies. Res Soc Work Pract 2010;20(2):137-160.

[31] Madson MB, Loignon AC, Lane C., Training in motivational interviewing: a systematic review. J Subst Abuse Treat 2009;36(1):101-109.

[32] Mertens VC, Goossens ME, Verbunt JA, Koke AJ, Smeets RJ., Effects of nurse-led motivational interviewing of patients with chronic musculoskeletal pain in preparation of rehabilitation treatment (PREPARE) on societal participation, attendance level, and cost-effectiveness: study protocol for a randomized controlled trial. Trials 2013;14(1):90.

[33] Miller W, Yahne C, Moyers T, Martinez J, Pirritano M., A randomized trial of methods to help clinicians learn motivational interviewing. J Consult Clin Psychol 2004;72(6):1050-1062. 
[34] Miller WR, Moyers TB., Eight stages in learning motivational interviewing. J Teach Addict 2007;5(1):3-17.

[35] Miller WR, Rollnick S. Ten things that motivational interviewing is not. Behav Cogn Psychother 2009;37(2):129-140.

[36] Miller WR, Rollnick S., The effectiveness and ineffectiveness of complex behavioral interventions: Impact of treatment fidelity. Contemp Clin Trials 2014;37(2):234-241.

[37] Peters J, Large RG, Elkind G., Follow-up results from a randomised controlled trial evaluating in- and outpatient pain management programmes. Pain 1992;50(1):41-50.

[38] Petrie KJ, Frampton T, Large RG, Moss-Morris R, Johnson M, Meechan G., What do patients expect from their first visit to a pain clinic? Clin J Pain 2005;21(4):297-301.

[39] Pollard CA., Preliminary validity study of the Pain Disability Index. Percept Mot Skills 1984;59:974.

[40] Post MW, Van der Zee CH, Hennink J, Schafrat CG, Visser Meily JM, Van Berlekom SB. Validity of the Utrecht Scale for Evaluation of Rehabilitation-Participation. Disabil Rehabil 2012;34(6):478-85

[41] Rainville J, Ahern DK, Phalen L., Altering beliefs about pain and impairment in a functionally oriented treatment program for chronic low back pain. Clin J Pain 1993;9(3):196-201.

[42] Rubak S, Sandbaek A, Lauritzen T, Christensen B., Motivational interviewing: a systematic review and meta-analysis. Br J Gen Pract 2005;55(513):305-312.

[43] Ryan RM, Plant RW, O'Malley S. Initial motivations for alcohol treatment: Relations with patient characteristics, treatment involvement, and dropout. Addict Behav 1995;20(3):279-297.

[44] Scascighini L, Toma V, Dober-Spielmann S, Sprott H., Multidisciplinary treatment for chronic pain: a systematic review of interventions and outcomes. Rheumatology (Oxford) 2008;47(5):670-678.

[45] Schulz KF, Altman DG, Moher D., CONSORT 2010 Statement: updated guidelines for reporting parallel group randomised trials. Trials 2010;11:32. 
[46] Schwarzer R., Jerusalem M., Generalized Self-Efficacy scale. In: J. Weinman, S. Wright, M. Johnston, editors. Measures in health psychology: A user's portfolio. Causal and control beliefs. Windsor, UK: Nfer-Nelson, 1995. pp. 35-37.

[47] Sloots M, Dekker JH, Pont M, Bartels EA, Geertzen JH, Dekker J., Reasons of drop-out from rehabilitation in patients of Turkish and Moroccan origin with chronic low back pain in The Netherlands: a qualitative study. J Rehabil Med 2010;42(6):566-573.

[48] Sloots M, Scheppers EF, Van de Weg FB, Dekker JH, Bartels EA, Geertzen JH, Dekker J., Higher dropout rate in non-native patients than in native patients in rehabilitation in The Netherlands. Int J Rehabil Res 2009;32(3):232-237.

[49] Smeets RJ, Beelen S, Goossens ME, Schouten EG, Knottnerus JA, Vlaeyen JW., Treatment expectancy and credibility are associated with the outcome of both physical and cognitive-behavioral treatment in chronic low back pain.

Clin J Pain 2008;24(4):305-315.

[50] Söderlund LL, Nilsen P, Kristensson M., Learning motivational interviewing: Exploring primary health care nurses' training and counselling experiences. Health Educ J 2008;67(2):102-109.

[51] Soer R, Reneman MF, Vroomen PC, Stegeman P, Coppes MH., Responsiveness and minimal clinically important change of the Pain Disability Index in patients with chronic back pain. Spine (Phila Pa 1976) 2012;37(8):711-715.

[52] Turk DC, Wilson HD, Cahana A., Treatment of chronic non-cancer pain. Lancet 2011;377(9784):2226-2235.

[53] Van der Zee CH, Kap A, Rambaran Mishre RR, Schouten E, Post MW., Responsiveness of four participation measures for outcomes of outpatient rehabilitation.

J Rehabil Med 2011;43:1003-1009.

[54] Van der Zee CH, Post MW, Brinkhof MW, Wagenaar RC., Comparison of the Utrecht Scale for Evaluation of Rehabilitation-Participation with the ICF Measure of Participation and Activities Screener and the WHO Disability Assessment Schedule II in persons with spinal cord injury. Arch Phys Med Rehabil 2014;95(1):87-93.

[55] Van der Zee CH, Priesterbach AR, Van der Dussen L, Kap A, Schepers VP, Visser Meily JM, Post MW., Reproducibility of three self-report participation measures: The ICF Measure of Participation and Activities Screener, the Participation Scale, and the Utrecht Scale for Evaluation of Rehabilitation-Participation. J Rehabil Med 2010;42(8):752-757. 
[56] Vong SK, Cheing GL, Chan F, So EM, Chan CC., Motivational enhancement therapy in addition to physical therapy improves motivational factors and treatment outcomes in people with low back pain: a randomized controlled trial. Arch Phys Med Rehabil 2011;92(2):176-183.

[57] Williams A, Eccleston C, Morley S., Psychological therapies for the management of chronic pain (excluding headache) in adults. Cochrane Database Syst Rev 2013(11):CD007407.

[58] Winter F., De pijn de baas [Mastering pain]. Soest: Ruitenberg Boek, 2008. 


\section{Abstract}

Treatment expectancy is important for the ultimate treatment outcome. The content validity of the Credibility and Expectancy Questionnaire (CEO) is unknown.

\section{Objectives}

To assess the content validity of the CEO and to test interpretations and responses to evaluate the need for adaptations of the CEO from a sample of chronic pain patients in different phases of their treatment.

\section{Methods}

Study design: Using the Three-Step Test-Interview (TSTI) method, a qualitative observational method. An iterative approach was applied in rounds of interviews, analyses and adaptations.

Study population: Chronic pain patients who had participated in a randomised controlled trial (RCT) to assess the additional effect of two types of pre-treatment on multidisciplinary pain treatment and who had given informed consent to be contacted for future research were asked to participate in the present study.

Main study parameters: Interpretations and responses of the participants to the CEO and the identification of response problems.

\section{Results}

Based on cognitive interviews of 17 participants and through subsequent adaptations of the CEO in 5 rounds, the instruction text of the CEO was changed. The CEO became an easy to understand and content-valid questionnaire for patients waiting for treatment. Participants who had already undergone treatment continued to have interpretation and response problems, but after adapting several references and time frames, it proved to be a content-valid questionnaire.

\section{Discussion}

Chronic pain patients waiting for rehabilitation treatment interpreted and responded to the CEO as intended. For patients who had already undergone rehabilitation treatment, the CEO needed to be improved considerably. 


\section{Introduction}

Chronic non-specific musculoskeletal pain is a major health burden. It occurs in approximately $10 \%$ of the general population [1] and contributes to disability [2], medical expenses [3], and a high amount of work absenteeism [4].

In the Netherlands, cognitive behavioral therapy (CBT)-based approaches are part of the regular rehabilitation care for patients with non-specific chronic pain [5] and are also recommended in Dutch national guidelines [6]. Rather than relieving pain, CBT aims to teach the patient to cope with pain, with the ultimate goal of increasing the patient's level of participation in society and quality of life [7].

The influential role of expectancy on therapy outcome has been confirmed in several CBT-based pain treatments [8-10] and in a meta-analysis of psychotherapy [11].

The concepts of credibility and expectancy are used interchangeably (e.g. [12]), though different levels of association with outcome have been shown [13]. Therefore, it may be functionally important to take expectancy as well as credibility into account, although expectancy seems to be a better predictor than credibility. Credibility has been defined as "how believable, convincing, and logical the treatment is", whereas expectancy refers to "improvements that clients believe will be achieved on the basis of a particular treatment" (Kazdin, 1979, p. 82) [14]. Devilly and Borkovec (2000) mention that expectancy stems from an affective base, whereas credibility is more cognitively based [13]. In questionnaires such as the CEO, these are often referred to as "feel" ("similar to those involved in hope or faith" ([13], p. 75)) and "think", respectively [13].

In the present study, we refer to "expectancy" while also incorporating "credibility", as Devilly and Borkovec mention that those concepts are usually used interchangeably [13].

Evidence from previous studies shows that treatment expectancy is a strong predictor of treatment outcome [12], and has it has been postulated that expectancy is a modifiable variable of treatments [12]. Even in chronic pain patients, expectancy can change over time [9] and seems to be modifiable $[10,15]$. Expectancy is also a predictor of long-term outcomes [9]. The whole process regarding changes in expectancy is not yet fully understood, and it is also uncertain whether a single measurement of expectancy can encapsulate the entire construct of expectancy for the whole treatment period [16]. Furthermore, it is not fully understood when expectancy needs to be measured [16]. Therefore, it is postulated that multiple measurements are needed throughout the duration of treatment to assess possible changes in expectancy [16]. Thus, considering the concept of expectancy as a process variable, it is until now unknown whether changes in expectancy are accountable for the ultimate treatment outcomes (e.g. [9, 16]).

As systematic reviews already have shown, there are several instruments or methods for measuring the concept of expectancy [16, 17], but the inconsistent use of valid measurement instruments is criticized [16]. Furthermore, information regarding development and testing, and reliability and validity is frequently omitted [17]. Due to this, there is an urgent need for a standardized and valid method or tool $[15,16]$ that can be used to measure the level of expectancy at multiple times. Currently, there is no questionnaire available that can do this.

An adapted and translated version of the Credibility and Expectancy Questionnaire (CEO) of 
Devilly and Borkovec (2000) [13] is often used in pain rehabilitation settings [10, 18]. The translated and modified Dutch version of the CEQ [18] consists of five items; three items are related to credibility and general expectancy regarding the treatment (part one), and two items are related to measuring the specific expectancy regarding the patient's own pain rehabilitation treatment (part two). The CEQ's wording of the items is adapted to the actual treatment objective. For example, in pain rehabilitation, the patient's expectations regarding treatment in general are rated, as well as, for example, the expected success in terms of decrease in disability. The CEQ has good psychometric properties (internal consistency and test-retest reliability), and the two-factor structure has been confirmed [10].

However, the content validity of this questionnaire is currently unknown. It is of critical importance to be able to properly interpret the actual meaning of the outcomes derived from a questionnaire [19], and to examine inconsistent interpretations, incomplete concept coverage, and misunderstandings [20] in relevant target groups at different stages of their treatment. Several best practices exist, and an iterative approach is recommended [21-24] to establish content validity in new or existing questionnaires.

This study aims to assess the content validity of the CEO and tests potentially necessary adaptations of the questionnaire by obtaining perspectives from a diverse sample of chronic pain patients who are at different stages of treatment.

\section{Research Questions}

In this study, the following research questions will be answered:

1a. How do chronic pain patients before and during rehabilitation treatment interpret and respond to the items of the Credibility and Expectancy Questionnaire (CEO)?

1b. If response problems are encountered during the completion of the CEQ, which adaptations in the items and/or instructions should be made in order to improve the validity of the CEQ? 


\section{Participants and Methods}

\section{Design}

A qualitative observational study design [25] was used. A specific form of cognitive interviewing (described in detail in the interview procedure section), the Three-Step Test-Interview (TSTI) method, was used. An advantage therein is that two subtypes of cognitive interview methods, think-aloud interviewing and probing techniques, were combined [26]. Think-aloud interviewing means that as a questionnaire is filled in, the cognitive processes are simultaneously verbalized by the participant. Probing strives to get more insight into the understanding and the retrieval or judgment process by having an interviewer asking for more specific information [26].

This interview technique combines three steps [27]. The first step is observing response behavior [28] (e.g. how a participant chooses a certain response option) and concurrent verbalization while the participant answers self-report questionnaires. The second step involves follow-up probing into specific aspects of this response process (e.g. the verbalization of formulations in the questionnaires which are difficult to understand). In the third step, the experiences and opinions of the participant are elicited [27].

\section{Participant selection/sampling}

This study involved seventeen participants with non-specific chronic musculoskeletal pain or fibromyalgia who participated in an earlier RCT, the Pre-Pain Rehabilitation Study (PREPARE; total $n=163$ ) [29], and who agreed to be contacted for related research. The inclusion and exclusion criteria for the current study were identical to those of the clinical trial [29]. A convenient sample frame [30] was used in the first instance, meaning that all persons who were available were approached (without balancing specific entry requirements). This choice was made to strive towards a broad dispersion of the participants' demographic characteristics, functioning levels and treatment phases.

We saw during the first interview round that participants who had finished the rehabilitation treatment encountered specific problems with regard to time-frame and recall, despite the fact that the patients who had finished treatment had also participated in the abovementioned RCT and had filled out the CEO before treatment started (for details see the Results section). Therefore, we first approached participants who had finished their treatment. We asked them to im-

agine their situation before or while just beginning the treatment so that we could attempt to resolve the response problems in the questionnaire for this group.

At the time the interviews were conducted, 12 participants had already finished their pain rehabilitation treatment, two were on the waiting list, one was undergoing treatment, one discontinued treatment prematurely, and one had never undergone the treatment (see also table 1).

\section{Ethics}

The Medical Ethics Committee of Maastricht Academic Hospital and Maastricht University approved the study protocol (NL38037.068.11). All participants provided written informed consent. 


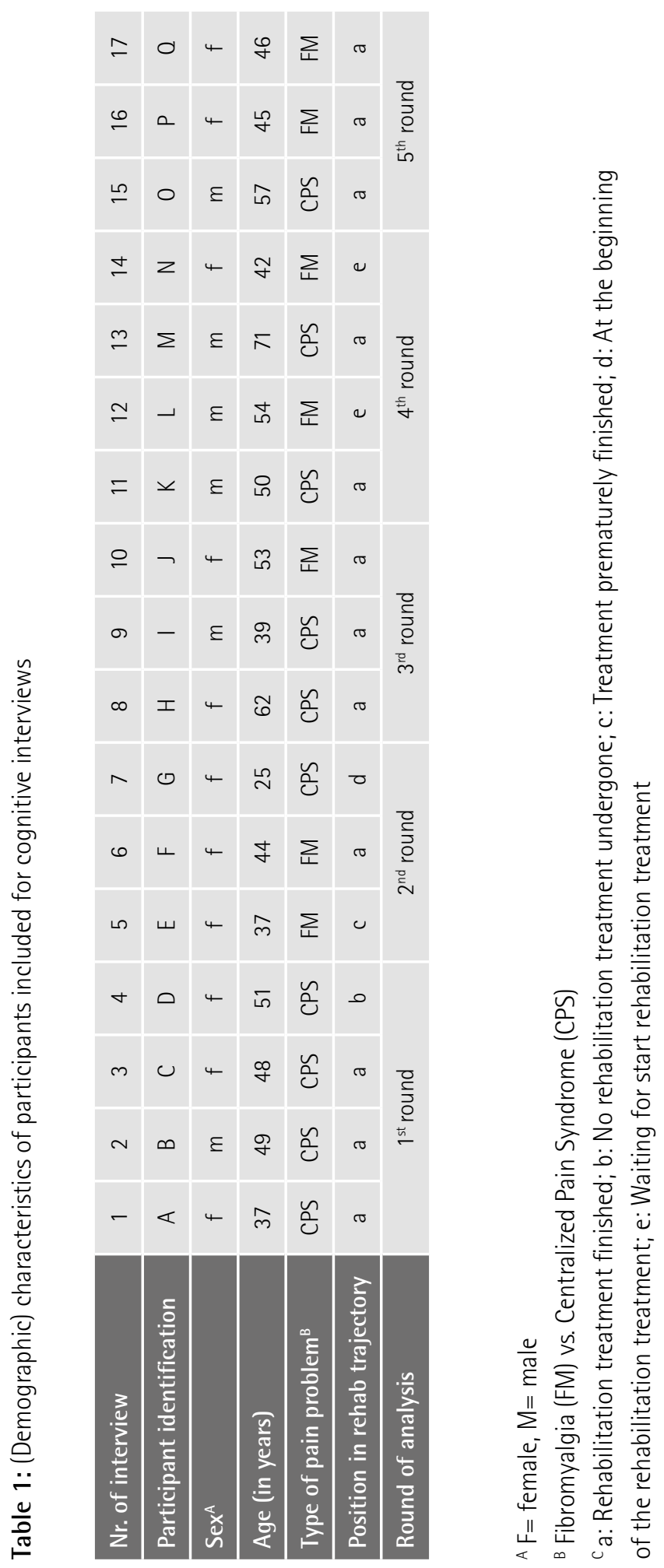




\section{Interview procedure and observations}

Think-aloud interviews using the TSTI method were conducted at the participants' homes to come as close as possible to the real-life situation in which the patient-related measurement instrument is completed $[27,31]$. One participant preferred to be interviewed at Maastricht University. A pilot interview with one participant was conducted to test the interview procedure, the interview guide and interview probes. The data from this interview were used in the analysis. The interview guide contained questions related to the interpretations and responses of the participants regarding the CEO and the identification of response problems in concordance with TSTI. Probing questions were used to gain in-depth information (see table 2).

Each participant was interviewed once, and all interviews were audio taped.

As thinking aloud is not a common activity in daily life, a grounded explanation and preliminary training was necessary to elicit a sufficient amount of think-aloud behavior [26]. Therefore, the participants were asked to first practice this by thinking aloud and filling in a questionnaire unrelated to this study. Then the actual think-aloud interview with the CEO followed.

The participants were given the following instructions: "Try to speak aloud everything that comes to your mind while you complete the questionnaire."

Directly after the completion of the CEO (version appendix 1) and while the patient was asked to think aloud, probing questions were asked to reveal more information regarding his or her thought processes. Examples of the probing questions can be found in table 2 .

Field notes [33] were made during and after every interview to document the interviewer's impressions of the interview and the interview process.

As an imperative [21], data collection and analysis happened in an iterative way, and themes that emerged during the sequential analysis were added in the interview guidelines and were verified in the subsequent interviews by asking probing questions specific to these themes. For instance, we added questions concerning the meaning of the word "credibility" and opinions regarding the scoring options (see the results section for details).

The interviews were conducted by a trained researcher and a trained research assistant (V.-C.M. and A.D.). The interview lasted approximately one hour. All interviews were audio taped.

\section{Main parameters}

Content validity is about how the respondent interprets the items of the questionnaire and decides on an answer [32]. If an assessment of content validity is dismissed, it could be because the concepts that are intended to be measured are not embodied in the actual questionnaire or are understood by the respondents differently than intended [33]. Therefore, based on the TSTI, the interviews focused on two main parameters [28]: the interpretations and responses of the participants and the identification of response problems.

\section{Interpretations and responses of the participants}

Cognitive interviewing pays particular attention to the underlying cognitive processes respondents use to answer items in a questionnaire [20]. The 4-stage model [34] is a common representation used for understanding this preprocess. According to this model, four actions have to be 
Table 2: Cognitive components and specific interview probes based on Tourangeau (1984), Bloem et al. (2008), Patrick et al. (2011), and Liu et al. (2011)

\begin{tabular}{|c|c|c|c|c|}
\hline $\begin{array}{l}\text { Cognitive } \\
\text { component }\end{array}$ & Definition & & Goal & $\begin{array}{l}\text { Interview probes incorporated } \\
\text { into interview guidelines }\end{array}$ \\
\hline \multirow[t]{4}{*}{$\begin{array}{l}\text { Com- } \\
\text { prehension }\end{array}$} & \multirow[t]{4}{*}{$\begin{array}{l}\text { Whether the respon- } \\
\text { dent understands } \\
\text { the question in the } \\
\text { way the researcher } \\
\text { intended them to. }\end{array}$} & Instruction & $\begin{array}{l}\text { To understand the } \\
\text { participant's inter- } \\
\text { pretation of the task } \\
\text { to be performed. }\end{array}$ & $\begin{array}{l}\text { Can you tell me in your own } \\
\text { words what the instructions are } \\
\text { asking you to do? } \\
\text { Can you describe any confusion/ } \\
\text { difficulty you had in understan- } \\
\text { ding the instructions? }\end{array}$ \\
\hline & & \multirow[t]{2}{*}{$\begin{array}{l}\text { Interpreta- } \\
\text { bility }\end{array}$} & $\begin{array}{l}\text { Understanding the } \\
\text { (phrasing of the) } \\
\text { survey questions. }\end{array}$ & $\begin{array}{l}\text { What does the target construct } \\
\text { in item (e.g. participation) mean } \\
\text { to you? } \\
\text { Is the phrasing of the specific } \\
\text { items in the questionnaire clear } \\
\text { to you? }\end{array}$ \\
\hline & & & $\begin{array}{l}\text { Understanding the } \\
\text { response options. }\end{array}$ & $\begin{array}{l}\text { What do the response scores } \\
\text { and accompanying text mean } \\
\text { to you? }\end{array}$ \\
\hline & & Paraphrasing & $\begin{array}{l}\text { To identify compre- } \\
\text { hension problems. }\end{array}$ & $\begin{array}{l}\text { Can you explain this term/ques- } \\
\text { tion in your own words? } \\
\text { Can you suggest any changes } \\
\text { that would improve the questi- } \\
\text { onnaire's wording? }\end{array}$ \\
\hline \multirow[t]{3}{*}{ Retrieval } & \multirow[t]{3}{*}{$\begin{array}{l}\text { Recalling relevant } \\
\text { information. }\end{array}$} & Recall & $\begin{array}{l}\text { To identify how } \\
\text { patients retrieve } \\
\text { information, or } \\
\text { remember situations } \\
\text { or events. }\end{array}$ & $\begin{array}{l}\text { How did you remember that? } \\
\text { What period of time did you } \\
\text { think about when you were } \\
\text { completing the questionnaire? }\end{array}$ \\
\hline & & Timeframe & & $\begin{array}{l}\text { Did you have a particular time- } \\
\text { frame in mind? } \\
\text { Which times did you compare in } \\
\text { order to choose an answer? } \\
\text { What does the timeframe mean } \\
\text { to you? }\end{array}$ \\
\hline & & $\begin{array}{l}\text { Standards of } \\
\text { comparison }\end{array}$ & $\begin{array}{l}\text { To explore the man- } \\
\text { ner of comparisons } \\
\text { made. }\end{array}$ & $\begin{array}{l}\text { Did you compare yourself to } \\
\text { someone or something else? }\end{array}$ \\
\hline Judgment & $\begin{array}{l}\text { Making decisions } \\
\text { regarding the } \\
\text { response. }\end{array}$ & & & $\begin{array}{l}\text { Can you explain how you } \\
\text { decided to choose this response } \\
\text { option? }\end{array}$ \\
\hline \multirow[t]{2}{*}{$\begin{array}{l}\text { Reporting and } \\
\text { response }\end{array}$} & \multirow[t]{2}{*}{$\begin{array}{l}\text { Matching answers to } \\
\text { an available response } \\
\text { option. }\end{array}$} & $\begin{array}{l}\text { Formatting } \\
\text { response }\end{array}$ & & $\begin{array}{l}\text { Were you able to find a response } \\
\text { option for the first answer that } \\
\text { came to your mind? }\end{array}$ \\
\hline & & $\begin{array}{l}\text { Response } \\
\text { options }\end{array}$ & $\begin{array}{l}\text { To understand how } \\
\text { participants interpret } \\
\text { the response options. }\end{array}$ & $\begin{array}{l}\text { What caused you to choose this } \\
\text { response option? And why did } \\
\text { you not choose a higher/lower } \\
\text { score? }\end{array}$ \\
\hline
\end{tabular}




\begin{tabular}{|c|c|c|c|c|}
\hline $\begin{array}{l}\text { Cognitive } \\
\text { component }\end{array}$ & Definition & & Goal & $\begin{array}{l}\text { Interview probes incorporated } \\
\text { into interview guidelines }\end{array}$ \\
\hline \multirow[t]{3}{*}{$\begin{array}{l}\text { Reporting and } \\
\text { response }\end{array}$} & \multirow[t]{3}{*}{$\begin{array}{l}\text { Matching answers to } \\
\text { an available response } \\
\text { option. }\end{array}$} & $\begin{array}{l}\text { Response } \\
\text { selection }\end{array}$ & $\begin{array}{l}\text { To understand how } \\
\text { participants make } \\
\text { decisions around } \\
\text { response choice. }\end{array}$ & $\begin{array}{l}\text { Could you tell me how you } \\
\text { decided on this response option } \\
\text { and not another one, e.g. XY } \\
\text { (mention alternative response } \\
\text { option)? }\end{array}$ \\
\hline & & $\begin{array}{l}\text { Considerati- } \\
\text { on of other } \\
\text { response } \\
\text { categories }\end{array}$ & $\begin{array}{l}\text { To identify respon- } \\
\text { dent difficulties with } \\
\text { the presentation of } \\
\text { the questionnaire. }\end{array}$ & $\begin{array}{l}\text { Did you also consider another } \\
\text { response option? If so, why did } \\
\text { you consider this? } \\
\text { Did the specified response } \\
\text { options fit your answer? }\end{array}$ \\
\hline & & $\begin{array}{l}\text { Content } \\
\text { coverage }\end{array}$ & $\begin{array}{l}\text { To ensure that } \\
\text { there are no missing } \\
\text { concepts. }\end{array}$ & $\begin{array}{l}\text { What other experiences do } \\
\text { you have with the concepts of } \\
\text { credibility and expectancy that } \\
\text { are not covered in this questi- } \\
\text { onnaire? }\end{array}$ \\
\hline \multirow[t]{3}{*}{ Preference } & & & & $\begin{array}{l}\text { Which part of the questionnaire } \\
\text { were you more comfortable with } \\
\text { answering? }\end{array}$ \\
\hline & & Format & $\begin{array}{l}\text { To identify partici- } \\
\text { pant's difficulty with } \\
\text { the presentation of } \\
\text { the questionnaire. }\end{array}$ & $\begin{array}{l}\text { What is your opinion about the } \\
\text { format? } \\
\text { Dou you have any suggestions } \\
\text { for improvement? }\end{array}$ \\
\hline & & Length & $\begin{array}{l}\text { To determine if the } \\
\text { length of time it } \\
\text { takes to complete } \\
\text { the questionnaire is } \\
\text { reasonable. }\end{array}$ & $\begin{array}{l}\text { What do you think about the } \\
\text { amount of time it takes to com- } \\
\text { plete the questionnaire? }\end{array}$ \\
\hline $\begin{array}{l}\text { Editing the } \\
\text { response }\end{array}$ & $\begin{array}{l}\text { Matching answer to } \\
\text { an available response } \\
\text { option. }\end{array}$ & & & $\begin{array}{l}\text { How did you make sure that your } \\
\text { answer fits with the available } \\
\text { response options? } \\
\text { In the case of response problems: } \\
\text { How would you change the } \\
\text { questionnaire so that your } \\
\text { answer fits with the available } \\
\text { response options? }\end{array}$ \\
\hline
\end{tabular}


fulfilled in order to answer a question: comprehending the question, retrieving necessary information, making a judgment and responding to the question. Comprehension is about "whether the respondent understands the question in the same way as the researcher intended" ([20], p. 232).

Related cognitive components, such as "standard of comparison", are used in comparable studies to assess the content validity of a questionnaire $[19,35,36]$. An overview of the cognitive components and specific probes that are used in the interview guideline to explore those processes in depth are presented in table 2.

\section{Identification of response problems}

While answering the questions in the $\mathrm{CEO}$, potential problems were explored (e.g. problematic formulations or the lack of a time frame). Those explorations led to insights into a potential mismatch between the meaning behind the items of the questionnaire and the participant's behaviour in answering those items.

\section{The evaluated questionnaire: The Credibility and Expectancy Questionnaire (CEO)}

The CEO that was used in the PREPARE study was adapted from the CEO from de Leeuw et al. (2008) [18], who had adapted the original version from Devilly et al. (2000) to be used in chronic pain rehabilitation. The CEO (to be found in appendix 1) consists of an explanatory preface, the instructions for part 1, part 1, the instructions for part 2, and part 2. Part 1 is related to current expectancy and credibility regarding the rehabilitation treatment in general. Part 2 is related to the specific personal situation. The CEO uses a Likert scale, ranging from 1 (not at all) to 9 (very much).

The CEQ's wording of the items is adapted to the actual treatment objective. Therefore, in pain rehabilitation, expectations about pain rehabilitation in general and the expected success in terms of the patient's individual increase in participation, decrease in disability, and decrease in pain intensity are rated. To clarify the meaning of the term participation for the respondents of the PREPARE study, an explanation was added to the CEQ in a footnote (see also appendix 1).

\section{Data Analysis}

For the analysis, three types of data were collected and used: The verbatim interview transcriptions, the field notes and the completed CEO questionnaires. Data were analyzed using a directed content analysis [37] and constant comparison [38] by searching for supportive and disconfirming data.

Based on earlier content analysis studies $[20,28,36]$, our coding scheme comprised of a system of categories and definitions regarding the cognitive components (see table 2). The coding scheme 
was applied to the participants' responses to the questionnaire items.

Furthermore, analysis took place in a cyclical process: Interviews and analysis alternated with meetings of the project team (first, second, and last author; details omitted for double-blind reviewing) to discuss the findings together (for a detailed overview, see figure 1), resulting in several adaptations of the analysis scheme.

Data collection and analysis were continued until data saturation was reached [21]. Data saturation occurred after 15 interviews. To confirm the findings, another two interviews were held. Nvivo9 software was used. A saturation grid can be found in table 3 .

Data triangulation, referring to the usage of different sources to increase validity [39], was achieved by including information from different sources such as interview transcripts and field notes, as well as by combining think-aloud interviews with subsequent probing questions. During the analysis, the project team (consisting of the first, second, and te last author; details omitted for double-blind reviewing) discussed the findings to strive for investigator triangulation [39].

Table 3: Saturation grid Identification of response problems

\begin{tabular}{|c|c|c|c|c|c|c|}
\hline & & \multicolumn{5}{|c|}{ Round in which response problem first appeared } \\
\hline & & Round 1 & Round 2 & Round 3 & Round 4 & Round 5 \\
\hline $\begin{array}{l}\text { Compre- } \\
\text { hension }\end{array}$ & Instruction & $x$ & & & & \\
\hline \multirow[t]{3}{*}{ Retrieval } & Recall & & & $x X$ & & \\
\hline & Time-frame & $x X$ & & & & \\
\hline & Standards of comparison & & $X X$ & & & \\
\hline Judgement & $\begin{array}{l}\text { Avoidance of extreme } \\
\text { values }\end{array}$ & $x$ & & & & \\
\hline $\begin{array}{l}\text { Reporting } \\
\text { and Res- } \\
\text { ponse }\end{array}$ & Response options & $X, X X$ & & & & \\
\hline others & $\begin{array}{l}\text { Difference pain / disability } \\
\text { resulting from pain }\end{array}$ & $x X$ & & & & \\
\hline
\end{tabular}

$x$ Participants waiting for treatment or just started treatment

xx Participants who already finished treatment 
Figure 1:

Flow chart cyclic process analysis and adaptation interview guideline and questionnaire

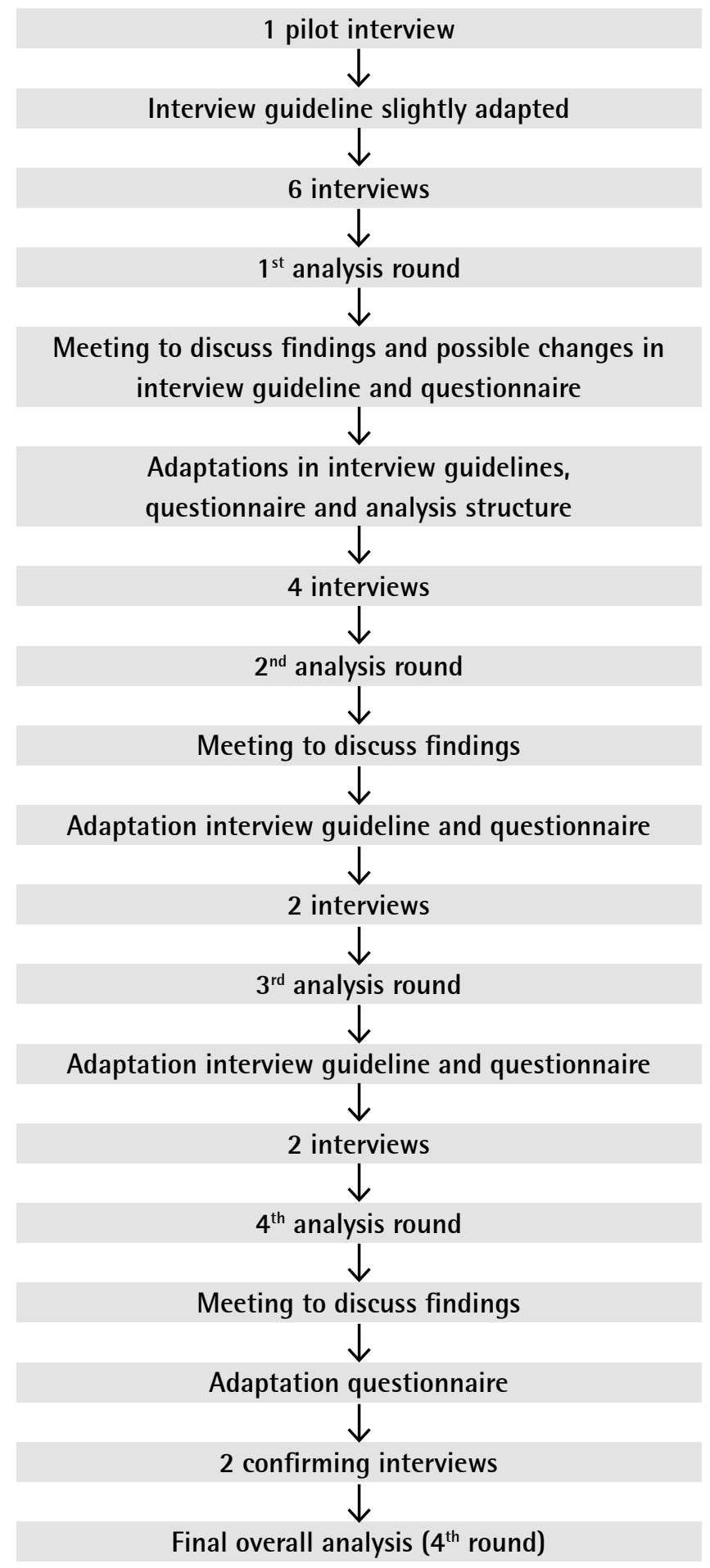




\section{Results}

The results of the 17 interviews are presented in order of the sub-items in the CEO and consist of five rounds of interviews and analysis (see also figure 1 and table 1).

The two main parameters, interpretations and responses of the participants (for its specific content refer to table 2) and identification of response problems, were used as structuring elements. This process was accompanied by making changes in the CEO (see also appendix 1) for each round.

\section{Round 1}

Interpretations and responses of the participants and identification of response problems Concerning the instruction text for part 1, all participants did not immediately know how the statement "in general" was referring to the request to describe problems in standard of comparison. Most participants solved this by filling in part 1 as if it was referring to their views "in general" regarding their own rehabilitation treatment rather than what was intended. Namely to describe their views regarding rehabilitation treatment of everyone "in general". All participants mentioned that they were not able to exclude their personal views while answering items regarding their expectancy "in general". Since the instruction text for part 2 referred to the specific personal situation, some participants perceived part 2 (and especially items 4a-c) as being redundant. Also, the participants did not know which reference of comparison to use.

All participants understood the word "participation" when asked about their expectations regarding increased participation. They could give a description, paraphrase it, and mention examples. However, none of the participants mentioned paid work as part of the concept of participation. Interestingly, all of their examples referred to free time, social contacts, and unpaid (voluntary) work. Also, the "quality of participation", which refers to a secondary aspect next to frequency, was part of the participants' definition of participation. By this, they meant that lowering the frequency of participation could improve the perceived quality of it.

Participants scored items regarding expectations of a "decrease in pain disability"' $(2 b, 4 b, 5 b)$ and a "decrease in pain intensity" (item 2c, 4c, 5c) differently, depending on whether they were scheduled on the waiting list or had already finished the pain rehabilitation treatment. Participants waiting for the treatment gave rather congruent scores (meaning the same numbers) for those items. Participants who had already finished treatment emphasized during the interview that the decrease in the disability-related items $(2 b, 4 b, 5 b)$ were scored in a non-congruent way. They differentiated between a decrease in disability resulting from pain and a decrease in pain as two entirely separate concepts. Participants mentioned that this process of separation was a result of the treatment they successfully underwent.

This difference became clearer to me during the therapy. In the past, I linked these more to each other; now I'm separating them. (participant I, has finished pain rehabilitation treatment). 
The rehabilitation treatment is very good. But again, the pain will remain. You have learned to deal with it even though the pain will always be there. (Quote participant $\mathrm{P}$, has finished FM treatment).

Since the general Dutch rating scale ranges from 1 to 10 and the CEQ's response options use a 9-point Likert scale, one participant experienced the questionnaire's response rating scale as rather unusual. In subsequent interviews, it became apparent that two participants had filled in the questionnaire as if a 10-point rating scale was used (participants $F, G$ ).

\section{Changes after round 1}

Due to the observed problems in the standard of comparison, the instruction texts were adapted. The instructions were reformulated to emphasize the participant's perspective on thoughts regarding rehabilitation treatment in general (part 1) and expectancies and expected success of the participant's own treatment specifically (part 2).

\section{Round 2}

Interpretations and responses of the participants

Participants who were at the beginning of the treatment period or were waiting for treatment interpreted and responded to the CEO as intended.

\section{Identification of response problems}

Another problem in the standard of comparison arose: in general, two different standards of comparison were used to answer the questionnaire's items. Those differed according to whether the participant was at the start of the treatment or had finished it. Participants prior to the start of the treatment were looking forward in terms of time in order to answer an item.

I do have certain expectations about how it will be afterwards compared to how it is now. This refers to how it usually is today and how I hope it will become [in the future]. Based on this, I have answered those questions. (Quote participant N, waiting for start treatment)

Participants who had finished the treatment encountered problems in choosing a suitable timeframe for answering the items in part 1 and part 2 because the reference timeframe had changed. The instructions for part 1 refer to the expected situation, and as a consequence the items in part 1 refer to the future. However, these participants had already finished the treatment: 
The phrasing of the question is confusing 'which is offered to you'. I have already finished it, but it mentions this moment right now.

(Quote participant I, has finished rehabilitation treatment)

\section{Changes after round 2}

As more problems were encountered by patients who had finished their treatment compared to patients at the beginning of the treatment, we developed a different version of the CEO for them. We named this version the CEQ-2. In this version, the wording of the items in terms of reference period (standard of comparison) was adapted by explicitly asking participants in the instruction to remember or imagine how they felt before or while just beginning the treatment.

\section{Round 3 and round 4}

\section{Identification of response problems}

Participants who had already undergone treatment still encountered problems with understanding what timeframe (recall period) was referred to in part 2 of the CEO-2. Participants were still uncertain regarding the reference period to compare with because they had already finished the rehabilitation treatment.

\section{[Frame of reference, part 2:] For this, I refer to after the treatment. [Frame of reference, part 2, item 4:] This is a question about what you think about the treatment and what you expect, even though my treatment is finished. It is about the time after treatment only. [Suggestion frame of reference:] I would mention somewhere in the questionnaire to look back in time when the treatment started (Quote participant 0, has finished rehabilitation treatment).}

\section{Changes after round 3 and round 4}

By defining the recall period more specifically for the subsequent participants, we found that changes in wording as well as in tense were necessary to increase the clarity regarding the specific manner of comparison. Specifically, the CEQ-2 differs from the original version of the CEO in the following points: the wording of the time frame was changed to present tense (items 1 , $2 a-c, 3)$; the words "attended treatment" were added (instructions for part 2, items $4 a-c$ ) to increase clarity; the recall period regarding expectations of improvement was adapted (items 5a-c). Therein, the CEO-2 asks patients to take themselves back in the time to before they started the treatment.

\section{Round 5}

Interpretations and responses of the participants

After implementing the changes in round 4, we found that the recall period was interpreted and responded to as intended by all of the participants, without confusion. 


\section{General observations throughout all rounds}

Two general observations we made throughout the entire interview process. The first is: when reasons for choosing a certain score were explored during probing questions, it became clear that several participants were inclined to avoid extreme values on the response option scale, indicating the probability of end-aversion bias. Participants wanted to avoid disappointments by having expectations that were too high, and participants who had not started the treatment yet argued that they were uncertain about what to expect as a result of the treatment. As a consequence, they choose scores in the middle of the possible range.

During the entire interview procedure, equipoise between participants regarding the preference for or against a 10-point Likert scale occurred (see round 2).

Furthermore, only one participant was able to express verbally that he could distinguish between the meanings of "to think" (in the participant's words "to expect") and "to hope":

I hope that my complaints lessen. But I do not expect anything. Hope is not what you expect. I have my expectations and I have my hope.

(Quote participant L, waiting for rehabilitation treatment) 


\section{Discussion and Conclusion}

The aim of this study was to assess the content validity of the CEO. The main result is that chronic pain patients waiting for or undergoing the rehabilitation treatment interpreted and responded to the CEO as intended.

Interviews with chronic pain patients waiting for or just beginning the rehabilitation treatment showed that only minimal changes in the wording of the instruction text for part 1 and part 2 of the CEQ were required. No other response problems could be identified. The only slightly necessary adaptations can be explained by the previous process of psychometric validation [13]. Furthermore, this indicates a direct link between some aspects of psychometric quality (test-rest reliability, factor structure, internal consistency, and the scale's ability to predict outcome) of the CEO resulting in the concept of credibility and expectancy and the actual meaning of the CEO as it is used in clinical or research settings.

For chronic pain patients who had already undergone rehabilitation treatment, we had to change the CEO considerably, resulting in the development of a second version of the CEO, the CEO- 2 . Already after round 2 we developed this version, which we tested in the three subsequent rounds. The CEQ-2 differs from the original version of the CEO in terms of the wording regarding the timeframes and adapted recall periods (see also appendix 2).

The application of the CEO in the context of participants at different stages of treatment highlights the urgent need for increasing our insight into the process of change in expectancy [16]. This is important, as the predicting role of expectancy is acknowledged [9] and can even be modified, so it needs to be assessed as a potential process measure of treatment success [10, 15]. The iterative TSTI approach worked like peeling an onion. We got through several layers before the underlying problems in interpretation, response, and understandability were encountered, unmasked, and ultimately solved. In each round, another problem was identified that had not been visible in the previous round. This provided depth to our study.

According to Nicklin et al. (2010), conducting cognitive interviews after the validation process of a questionnaire is completed would be too late to be able to make changes (e.g. in the wording) [23] that would increase its validity. Therefore, several authors recommend to use an iterative approach when developing a new questionnaire [21-24].

Saturation occurred after 15 interviews, and we feel that this indicates robust and complete findings and results in a content-valid measurement instrument.

We found that one participant was able to verbally express the difference in meaning between "think" (by mentioning "expect") and "hope", which was also differentiated in the concept approach of Devilly and Borkovec (2000) [13]. From our point of view, this indicates a successful translation and validation process and that there is no reason for concern that this specific aspect of the CEQ is not understood by patients.

Furthermore, we made an unexpected but confirmative determination of the discriminative ability of the CEO: The primary goal of cognitive behavioral therapy as it relates to pain rehabilitation is increasing activity and participation, rather than decreasing pain. This is achieved by having the 
patient reconceptualize pain and disability resulting from pain. We found that the questionnaire items regarding the therapeutic goals differentiated between participants who had already finished the treatment and those who were waiting for or had just started treatment.

We began recruiting participants by means of convenience sampling. This means that we interviewed the participants who came in first. This had the advantage that variability in the levels of functioning and the stages of treatment was present. However, by doing it this way, an oversampling of participants who had already finished the rehabilitation treatment occurred. A reason for oversampling was probably that we had no influence on the selection of the stage of treatment.

In the end, this resulted in the observation that participants who had finished treatment encountered specific problems interpreting and responding to the questionnaire. To explore this in more depth, we changed our sampling strategy to purposive sampling, which is commonly done to create variation in the confirming or disconfirming cases [21].

There is a need to untangle the concept of expectancy and to estimate when and how often expectancy needs to be measured. But first, a proper measurement tool is needed that can be used in longitudinal designs, for example [16]. The current study makes a first step in this direction by developing a content-valid questionnaire. We aimed to develop two versions: 1) that gives the opportunity to untangle the concept of expectancy at the start of and during treatment, and 2) a version that, for the participants who have already completed the treatment, taps into what their expectancy was before or at the beginning of the treatment (the CEO-2).

A next step should be the examination of the practical applicability in a research setting, for example by conducting studies on the correlation between the CEO-2 and the original version of the CEO, which was already indicated to be content valid. This is outside the focus of the current study.

Furthermore, in the future, particular attention should be paid to end-aversion bias (central tendency bias), which we observed during the cognitive interviews. If it is confirmed that patients filling in the CEO tend to avoid the upper or lower end of the rating scale, the adaptation of answer categories towards less absolute statements at the endpoints or the addition of 'throw away' categories, which would serve as anchors at the ends of the rating scale, should be reconsidered [40].

Along with Pool et al. (2010) [28], we recommend that qualitative approaches should be an integral part of the development of a questionnaire. A limitation is that this iterative approach is time consuming.

The TSTI approach is a useful tool for exploring the process of interpretation and response, and for identifying response problems in the CEO in a systematic way. More generally, qualitative methods have an added value in the improvement of questionnaires.

All in all, this cyclical process of cognitive interviews, analysis, and adaptation of the questionnaire was an advantageous approach in the current study. By doing so, we gained a deeper understanding of how the questionnaire was interpreted by the participants and thereby could improve its validity. 
As of now, the CEQ is a content-valid questionnaire with two versions: a minimally adapted version for patients waiting for or just beginning rehabilitation treatment, and an adapted version for patients who are undergoing or have finished the treatment.

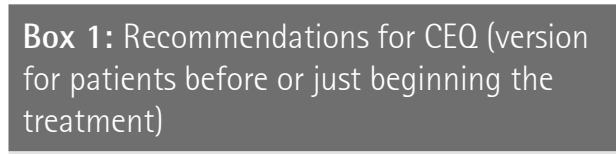

\section{Instructions}

Slight adaptations in the instructions text for part 1 and part 2
Box 2: Recommendations for CEQ-2 (for patients who are undergoing or have already finished the treatment)

\begin{tabular}{|l|l|}
$\begin{array}{l}\text { Change wording } \\
\text { regarding timeframe }\end{array}$ & Items 1, 2a-c, 3 \\
\hline $\begin{array}{l}\text { Add words "attended } \\
\text { treatment" }\end{array}$ & $\begin{array}{l}\text { Instructions for part } \\
\text { 2, items 4a-c }\end{array}$ \\
\hline Adapt recall period & $\begin{array}{l}\text { Check of compleet } \\
\text { Items 5a-c }\end{array}$ \\
\hline
\end{tabular}




\section{Questionnaire 1}

CEQ Prepare patient start of the treatment 


\section{Verwachtingsschaal}

\section{Instructie}

We willen $u$ vragen om op de volgende bladzijden aan te geven hoe sterk $u$ op dit moment gelooft dat de revalidatiebehandeling die $u$ mogelijk gaat volgen $u$ zal helpen in het leren verbeteren van uw deelname aan maatschappelijke activiteiten, het verminderen van beperkingen als gevolg van uw pijnklachten, en het verminderen van de pijn.

Onder deelname aan maatschappelijke activiteiten worden activiteiten verstaan zoals

(on)betaald werken, huishoudelijke taken of op bezoek gaan bij familie of vrienden.

Let op, het gaat hier alleen om het weer opnieuw deelnemen aan activiteiten die $u$ deed voordat $u$ pijnklachten had en vanwege uw pijnklachten niet meer doet of minder bent gaan doen.

De vragenlijst bestaat uit twee delen. In het eerste deel is het de bedoeling dat $u$ aangeeft hoe $u$ denkt over de revalidatiebehandeling. In het tweede deel is het de bedoeling dat u uw verwachtingen aangeeft over de behandeling en het mogelijke succes ervan. 


\section{Deel 1}

Voor dit eerste onderdeel is het de bedoeling dat $u$ aangeeft hoe $u$ denkt over de revalidatiebehandeling. Omcirkel bij elke uitspraak het cijfer dat het beste bij uw antwoord past.

1. Hoe geloofwaardig lijkt op dit moment de revalidatiebehandeling die $u$ aangeboden wordt?

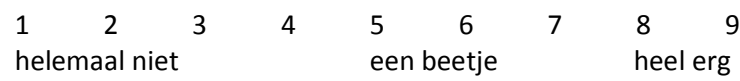

2. a. Hoe succesvol denkt u op dit moment dat de revalidatiebehandeling zal zijn in het verbeteren van uw deelname aan maatschappelijke activiteiten* ondanks de pijnklachten?

$\begin{array}{llllllllr}1 & 2 & 3 & 4 & 5 & 6 & 7 & 8 & 9 \\ \text { helemaal niet } & & & \text { een beetje } & & \text { heel erg }\end{array}$

b. Hoe succesvol denkt u op dit moment dat de revalidatiebehandeling zal zijn in het verminderen van uw beperkingen als gevolg van uw pijnklachten?

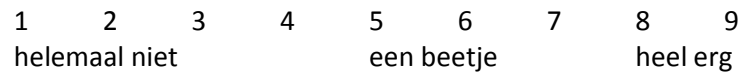

c. Hoe succesvol denkt u op dit moment dat de revalidatiebehandeling zal zijn in het verminderen van de pijnklachten?

$\begin{array}{lrllllllr}1 & 2 & 3 & 4 & 5 & 6 & 7 & 8 & 9 \\ \text { helemaal niet } & & & \text { een beetje } & & \text { heel erg }\end{array}$

3. Hoe zelfverzekerd zou u zijn in het aanraden van deze revalidatiebehandeling aan een vriend(in) met dezelfde klachten als $u$ ?

$\begin{array}{llllllllr}1 & 2 & 3 & 4 & 5 & 6 & 7 & 8 & 9 \\ \text { helemaal niet } & & & \text { een beetje } & & \text { heel erg }\end{array}$

* In de instructie van deze vragenlijst wordt uitgelegd wat verstaan wordt onder maatschappelijke activiteiten.

Versie 26-8-2013 
Deel 2

Voor dit tweede onderdeel is het de bedoeling dat u uw verwachtingen aangeeft ten aanzien van de revalidatiebehandeling en het mogelijke succes ervan.

Omcirkel bij elke uitspraak het cijfer dat het beste bij uw antwoord past.

4. a. Hoeveel verwacht $\mathbf{u}$ dat de revalidatiebehandeling $\mathbf{u}$ zal helpen in het verbeteren van uw deelname aan maatschappelijke activiteiten* ondanks de pijnklachten?

$\begin{array}{llllllllr}1 & 2 & 3 & 4 & 5 & 6 & 7 & 8 & 9 \\ \text { helemaal niet } & & & \begin{array}{l}\text { een beetje } \\ \text { heel erg }\end{array}\end{array}$

b. Hoeveel verwacht $\mathbf{u}$ dat de revalidatiebehandeling $\mathbf{u}$ zal helpen in het verminderen van uw beperkingen als gevolg van uw pijnklachten?

$\begin{array}{lrllllllr}1 & 2 & 3 & 4 & 5 & 6 & 7 & 8 & 9 \\ \text { helemaal niet } & & & \begin{array}{l}\text { een beetje } \\ \text { heel erg }\end{array}\end{array}$

c. Hoeveel verwacht $\mathbf{u}$ dat de revalidatiebehandeling $\mathbf{u}$ zal helpen in het verminderen van uw pijnklachten?

$\begin{array}{llllllllr}1 & 2 & 3 & 4 & 5 & 6 & 7 & 8 & 9 \\ \text { helemaal niet } & & & \text { een beetje } & & \text { heel erg }\end{array}$

5. a. Hoeveel verbetering in uw deelname aan maatschappelijke activiteiten* verwacht $\mathbf{u}$ aan het einde van de behandelperiode?

$\begin{array}{llllllllr}1 & 2 & 3 & 4 & 5 & 6 & 7 & 8 & 9 \\ \text { helemaal niet } & & & \text { een beetje } & & \begin{array}{l}\text { heel erg }\end{array}\end{array}$

b. Hoeveel verbetering in uw beperkingen verwacht $u$ aan het einde van de behandelperiode?

$\begin{array}{lllllcllr}1 & 2 & 3 & 4 & 5 & 6 & 7 & 8 & 9 \\ \text { helemaal niet } & & & \begin{array}{l}\text { een beetje } \\ \text { heel erg }\end{array}\end{array}$

Hoeveel vermindering in uw pijn verwacht $u$ aan het einde van de

c. behandelperiode?

$\begin{array}{llllllllr}1 & 2 & 3 & 4 & 5 & 6 & 7 & 8 & 9 \\ \text { helemaal geen } & & & & \text { een beetje }\end{array}$

* In de instructie van deze vragenlijst wordt uitgelegd wat verstaan wordt onder maatschappelijke activiteiten.

Versie 26-8-2013 


\section{Questionnaire 2}

CEQ Prepare patient end of the treatment 


\section{Verwachtingsschaal}

\section{Instructie}

We willen $u$ vragen om op de volgende bladzijden aan te geven hoe sterk $u$ op dit moment gelooft dat de revalidatiebehandeling die u gevolgd heeft $u$ zal helpen in het leren verbeteren van uw deelname aan maatschappelijke activiteiten, het verminderen van beperkingen als gevolg van uw pijnklachten, en het verminderen van de pijn.

Onder deelname aan maatschappelijke activiteiten worden activiteiten verstaan zoals

(on)betaald werken, huishoudelijke taken of op bezoek gaan bij familie of vrienden.

Let op, het gaat hier alleen om het weer opnieuw deelnemen aan activiteiten die $u$ deed voordat $u$ pijnklachten had en vanwege uw pijnklachten niet meer doet of minder bent gaan doen.

De vragenlijst bestaat uit twee delen. In het eerste deel is het de bedoeling dat $u$ aangeeft hoe $u$ denkt over de revalidatiebehandeling. In het tweede deel is het de bedoeling dat u uw verwachtingen aangeeft over de behandeling die u gevolgd heeft en het mogelijke succes ervan in de toekomst. 


\section{Deel 1}

Voor dit eerste onderdeel is het de bedoeling dat $u$ aangeeft hoe $u$ denkt over de revalidatiebehandeling. Omcirkel bij elke uitspraak het cijfer dat het beste bij uw antwoord past.

1. Hoe geloofwaardig lijkt op dit moment de revalidatiebehandeling?

$\begin{array}{lllllllll}1 & 2 & 3 & 4 & 5 & 6 & 7 & 8 & 9 \\ \text { helemaal niet } & & & & \text { een beetje } & & \text { heel erg }\end{array}$

2. a. Hoe succesvol denkt u op dit moment dat de revalidatiebehandeling is in het verbeteren van deelname aan maatschappelijke activiteiten* ondanks de pijnklachten?

$\begin{array}{llllllllr}1 & 2 & 3 & 4 & 5 & 6 & 7 & 8 & 9 \\ \text { helemaal niet } & & & \text { een beetje } & & \text { heel erg }\end{array}$

b. Hoe succesvol denkt u op dit moment dat de revalidatiebehandeling is in het verminderen van beperkingen als gevolg van uw pijnklachten?

$\begin{array}{llllllllr}1 & 2 & 3 & 4 & 5 & 6 & 7 & 8 & 9 \\ \text { helemaal niet } & & & \text { een beetje } & & \text { heel erg }\end{array}$

c. Hoe succesvol denkt u op dit moment dat de revalidatiebehandeling is in het verminderen van de pijnklachten?

$\begin{array}{lrllllllr}1 & 2 & 3 & 4 & 5 & 6 & 7 & 8 & 9 \\ \text { helemaal niet } & & & \text { een beetje } & & \text { heel erg }\end{array}$

3. Hoe zelfverzekerd bent $u$ in het aanraden van deze revalidatiebehandeling aan een vriend(in) met dezelfde klachten als $u$ ?

$\begin{array}{llllllllr}1 & 2 & 3 & 4 & 5 & 6 & 7 & 8 & 9 \\ \text { helemaal niet } & & & & \text { een beetje } & & \text { heel erg }\end{array}$

* In de instructie van deze vragenlijst wordt uitgelegd wat verstaan wordt onder maatschappelijke activiteiten.

Versie 8-10-2013 
Deel 2

Voor dit tweede onderdeel is het de bedoeling dat $u$ uw verwachtingen aangeeft ten aanzien van de revalidatiebehandeling die u gevolgd heeft en het mogelijke succes ervan in de toekomst.

Omcirkel bij elke uitspraak het cijfer dat het beste bij uw antwoord past.

4. a. Hoeveel verwacht $u$ dat de gevolgde revalidatiebehandeling $u$ zal helpen in het verbeteren van uw deelname aan maatschappelijke activiteiten* ondanks de pijnklachten?'

$\begin{array}{lrlllcllr}1 & 2 & 3 & 4 & 5 & 6 & 7 & 8 & 9 \\ \text { helemaal niet } & & & \text { een beetje } & & \text { heel erg }\end{array}$

b. Hoeveel verwacht $u$ dat de gevolgde revalidatiebehandeling $u$ zal helpen in het verminderen van uw beperkingen als gevolg van uw pijnklachten?'

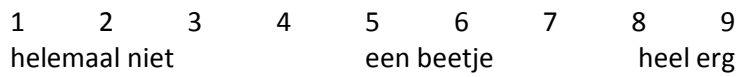

c. Hoeveel verwacht $u$ dat de gevolgde revalidatiebehandeling $u$ zal helpen in het verminderen van uw pijnklachten?'

$\begin{array}{lrllllllr}1 & 2 & 3 & 4 & 5 & 6 & 7 & 8 & 9 \\ \text { helemaal niet } & & & \text { een beetje } & & \text { heel erg }\end{array}$

5. Voordat u met de behandeling startte,

a. Hoeveel verbetering in uw deelname aan maatschappelijke activiteiten* verwachtte $\mathbf{u}$ ?

$\begin{array}{lrllllllr}1 & 2 & 3 & 4 & 5 & 6 & 7 & 8 & 9 \\ \text { helemaal niet } & & & \begin{array}{l}\text { een beetje } \\ \text { heel erg }\end{array}\end{array}$

b. Hoeveel verbetering in uw beperkingen verwachtte u?

$\begin{array}{lrllllllr}1 & 2 & 3 & 4 & 5 & 6 & 7 & 8 & 9 \\ \text { helemaal niet } & & & & \text { een beetje }\end{array}$

c. Hoeveel vermindering in uw pijn verwachtte $u$ ?

$\begin{array}{llllllllr}1 & 2 & 3 & 4 & 5 & 6 & 7 & 8 & 9 \\ \text { helemaal geen } & & & \text { een beetje } & & \text { heel erg }\end{array}$

* In de instructie van deze vragenlijst wordt uitgelegd wat verstaan wordt onder maatschappelijke activiteiten.

Versie 8-10-2013 


\section{References}

[1] Gran JT., The epidemiology of chronic generalized musculoskeletal pain.

Best Pract Res Clin Rheumatol 2003;17:547-561.

[2] Badley EM., Webster GK. and Rasooly I., The impact of musculoskeletal disorders in the population: are they just aches and pains? Findings from the 1990 Ontario Health Survey. J Rheumatol 1995;22:733-739.

[3] Meerding WJ., Bonneux L., Polder JJ., Koopmanschap MA. and van der Maas PJ., Demographic and epidemiological determinants of healthcare costs in Netherlands: cost of illness study. BMJ 1998;317:111-115.

[4] Koes BW., van Tulder MW. and Thomas S., Diagnosis and treatment of low back pain. BMJ 2006;332:1430-1434.

[5] Köke A., Consensus Rapport Pijnrevalidatie Nederland [Consensus Report Pain rehabilitation Netherlands]. Maastricht: Pijn Kennis Centrum Maastricht [Pain Knowledge Centre Maastricht], 2005.

[6] Bekkering GE., Hendriks HJM., Koes BW., Oostendorp RAB., Ostelo R., Thomassen JMC. and van Tulder MW., Dutch Physiotherapy Guidelines for Low Back Pain. Physiotherapy 2003;89:82-96.

[7] Turk DC., Wilson HD. and Cahana A., Treatment of chronic non-cancer pain. Lancet 2011;377:2226-2235.

[8] Broderick JE., Junghaenel DU., Schneider S., Bruckenthal P. and Keefe FJ., Treatment expectation for pain coping skills training: relationship to osteoarthritis patients' baseline psychosocial characteristics. Clin J Pain 2011;27:315-322.

[9] Goossens ME., Vlaeyen JW., Hidding A., Kole-Snijders A. and Evers SM., Treatment expectancy affects the outcome of cognitive-behavioral interventions in chronic pain. Clin J Pain 2005;21:18-26; discussion 69-72. 
[10] Smeets RJ., Beelen S., Goossens ME., Schouten EG., Knottnerus JA. and Vlaeyen JW., Treatment expectancy and credibility are associated with the outcome of both physical and cognitive-behavioral treatment in chronic low back pain. Clin J Pain 2008;24:305-315.

[11] Constantino MJ., Arnkoff DB., Glass CR., Ametrano RM. and Smith JZ., Expectations. J Clin Psychol 2011;67:184-192.

[12] Crow R., Gage H., Hampson S., Hart J., Kimber A. and Thomas H., The role of expectancies in the placebo effect and their use in the delivery of health care: a systematic review. Health Technol Assess 1999;3:1-96.

[13] Devilly GJ. and Borkovec TD., Psychometric properties of the credibility/expectancy questionnaire. J Behav Ther Exp Psychiatry 2000;31:73-86.

[14] Kazdin AE., Therapy outcome questions requiring control of credibility and treatmentgenerated expectancies. Behav Ther 1979;10:81-93.

[15] Bialosky JE., Bishop MD. and Cleland JA., Individual expectation: an overlooked, but pertinent, factor in the treatment of individuals experiencing musculoskeletal pain. Phys Ther 2010;90:1345-1355.

[16] Van Hartingsveld F., Ostelo RW., Cuijpers P., De Vos R., Riphagen, II and De Vet HC., Treatment-related and patient-related expectations of patients with musculoskeletal disorders: a systematic review of published measurement tools.

Clin J Pain 2010;26:470-488.

[17] Haanstra TM., van den Berg T., Ostelo RW., Poolman RW., Jansma EP., Cuijpers P. and de Vet HC., Systematic review: do patient expectations influence treatment outcomes in total knee and total hip arthroplasty? Health Qual Life Outcomes 2012;10:152.

[18] Leeuw M., Goossens ME., van Breukelen GJ., de Jong JR., Heuts PH., Smeets RJ., Koke AJ. and Vlaeyen JW., Exposure in vivo versus operant graded activity in chronic low back pain patients: results of a randomized controlled trial. Pain 2008;138:192-207.

[19] Patrick DL., Burke LB., Gwaltney CJ., Leidy NK., Martin ML., Molsen E. and Ring L., Content validity--establishing and reporting the evidence in newly developed patient-reported outcomes (PRO) instruments for medical product evaluation: ISPOR PRO Good Research Practices Task Force report: part 2--assessing respondent understanding. Value Health 2011;14:978-988. 
[20] Collins D., Pretesting survey instruments: an overview of cognitive methods. Qual Life Res 2003;12:229-238.

[21] Brod M., Tesler LE. and Christensen TL., Qualitative research and content validity: developing best practices based on science and experience. Qual Life Res 2009;18:1263-1278.

[22] Frost MH., Reeve BB., Liepa AM., Stauffer JW., Hays RD. and Mayo FDAP-ROCMG., What is sufficient evidence for the reliability and validity of patient-reported outcome measures? Value Health 2007;10 Suppl 2:S94-S105.

[23] Nicklin J., Cramp F., Kirwan J., Urban M. and Hewlett S., Collaboration with patients in the design of patient-reported outcome measures: capturing the experience of fatigue in rheumatoid arthritis. Arthritis Care Res 2010;62:1552-1558.

[24] Turner RR., Quittner AL., Parasuraman BM., Kallich JD., Cleeland CS. and Mayo FDAP-ROC MG., Patient-reported outcomes: instrument development and selection issues. Value Health 2007;10 Suppl 2:S86-93.

[25] Wollny A. and Marx G., Qualitative Sozialforschung-Ausgangspunkte und Ansätze für eine forschende Allgemeinmedizin. Teil 2: Qualitative Inhaltsanalyse vs. Grounded Theory. [Qualitative Social Research - Origins and Approaches for research in Family Medicine]. Z Allg Med 2009;85:467-476.

[26] Willis GB., Cognitive Interviewing. A Tool for Improving Questionnaire Design. Thousand Oaks: Sage Publications, 2005.

[27] Hak T., Van der Veer K. and Jansen H., The Three-Step Test-Interview (TSTI): An observational instrument for pretesting self-completion questionnaires. Surv Res Methods 2008;2:143-150.

[28] Pool JJ., Hiralal SR., Ostelo RW., van der Veer K. and de Vet HC., Added value of qualitative studies in the development of health related patient reported outcomes such as the Pain Coping and Cognition List in patients with sub-acute neck pain. Man Ther 2010;15:43-47.

[29] Mertens V-C., Goossens M., Verbunt J., Koke A. and Smeets R., Effects of nurse-led motivational interviewing of patients with chronic musculoskeletal pain in preparation of rehabilitation treatment (PREPARE) on societal participation, attendance level, and cost-effectiveness: study protocol for a randomized controlled trial. Trials 2013;14:90.

[30] Battaglia M., Convenience Sampling. In: Lavrakas PJ ed. Encyclopedia of Survey Research Methods Thousand Oaks, CA: SAGE Publications, Inc., 2008:Mike Battaglia. 
[31] Manual for the Three-Step test-Interview (TSTI).

[32] Patrick DL., Burke LB., Gwaltney CJ., Leidy NK., Martin ML., Molsen E. and Ring L. , Content validity--establishing and reporting the evidence in newly developed patientreported outcomes (PRO) instruments for medical product evaluation: ISPOR PRO good research practices task force report: part 1--eliciting concepts for a new PRO instrument. value Health 2011;14:967-977.

[33] Rothman M., Burke L., Erickson P., Leidy NK., Patrick DL. and Petrie CD., Use of Existing Patient-Reported Outcome (PRO) Instruments and Their Modification: The ISPOR Good Research Practices for Evaluating and Documenting Content Validity for the Use of Existing Instruments and Their Modification PRO Task Force Report. Value Health 2009;12:1075-1083.

[34] Tourangeau R., Cognitive sciences and survey methods. In: Jabine T., Straf M., Tanur J. and Tourangeau R. eds., Cognitive Aspects of Survey Methodology: Building a Bridge Between the Disciplines. Washington DC: National Academy Press, 1984:73-100.

[35] Liu R., Buffart L., Kersten MJ., Spiering M., Brug J., van Mechelen W. and Chinapaw M., Psychometric properties of two physical activity questionnaires, the AQuAA and the PASE, in cancer patients. BMC Med Res Methodol 2011;11:30.

[36] Bloem EF., van Zuuren FJ., Koeneman MA., Rapkin BD., Visser MRM., Koning CCE. and Sprangers MAG., Clarifying quality of life assessment: do theoretical models capture the underlying cognitive processes? Qual Life Res 2008;17:1093-1102.

[37] Hsieh H-F. and Shannon SE., Three Approaches to Qualitative Content Analysis. Qual Health Res 2005;15:1277-1288.

[38] Pope C. and Mays N., Reaching the parts other methods cannot reach: an introduction to qualitative methods in health and health services research. BMJ 1995;311:42-45.

[39] Merriam SB., Qualitative Research: A Guide to Design and Implementation. San Francisco, CA: Jossey-Bass, 2009.

[40] Streiner D. and Norman G., Health measurement scales: a practical guide to their development and use. New York: Oxford University Press, 1995. 


\title{
Chapter 8
}

\author{
GENERAL DISCUSSION
}

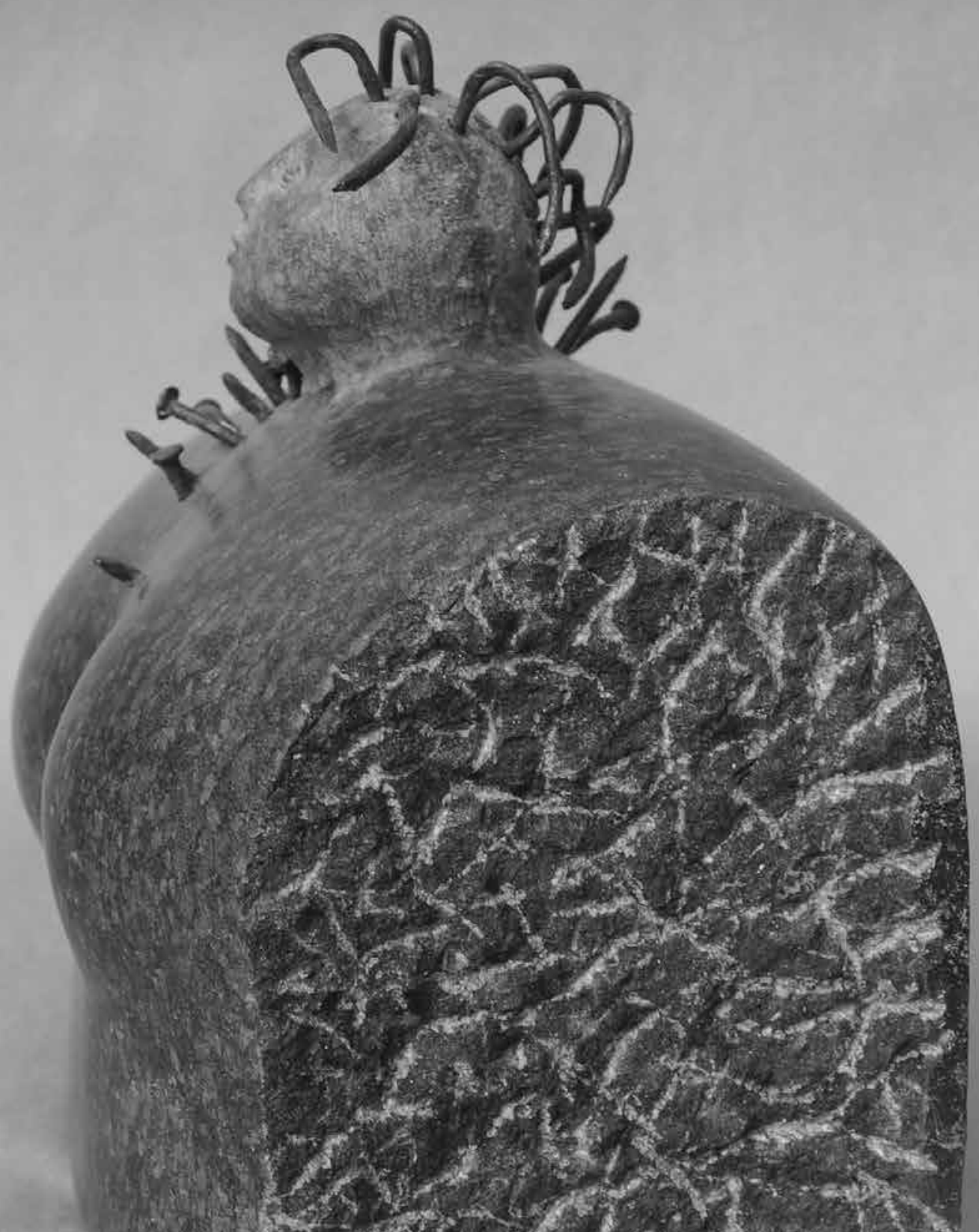




\section{General Discussion}

Multidisciplinary pain rehabilitation taking into account the biopsychosocial perspective of chronic pain shows moderate effectiveness. However, a low level of patient motivation and subsequent drop-out from treatment is present in a considerable number of patients. Probably, this occurs because the aim of the treatment is improvement of functioning and not pain reduction, which can be contrary to the patients' expectations.

The main objective of this current dissertation has been to evaluate whether Motivational Interviewing could be an effective mean to prepare patients with chronic pain for their subsequent pain rehabilitation treatment. The Pre-pain rehabilitation (Prepare) trial aimed therefore to compare the effectiveness of a nurse-led Motivational Interviewing-based pre-treatment (MIP) intervention and a nurse-led pain education (UC), in terms of social participation, pain-related disability, and drop-out from pain rehabilitation treatment. Furthermore, and related to this, additional aims were the examination of the feasibility of the pre-treatment and the determination of the quality of the intervention delivered. Another aim was to get insight in the experiences of the nurses and patients involved in order to reach a complementary picture of potential users and stakeholders of this pre-treatment intervention.

This general discussion aims to synthesize the empirical findings. These findings are discussed in the light of current scientific literature. Subsequently, general conclusions are drawn, strengths and limitations of the studies and methods used are discussed. Furthermore, methodological considerations as well as clinical implications are outlined, and future research directions will be sketched. 


\section{Effect evaluation: potential reasons and explanations for the findings}

The results of an effect evaluation (chapter 6) of the Ml-based pre-treatment in comparison to pain education showed that MI was not superior in terms of an increase in social participation, reduction of pain-related disability, and decrease of drop-out from treatment compared to pain education. However, a difference in Ml's effectiveness in subgroups and MI's effectiveness in terms of improvement of functioning was found.

As far, it is known from several meta-analyses that $\mathrm{Ml}$ is superior in divergent health behaviors and settings compared to no intervention, and as efficacious as other evidence-based interventions $[12,36,49,50,79]$. However, a recent meta-analysis reported upon MI's limited effectiveness regarding (medication) adherence [49].

In the field of musculoskeletal health care, some studies indicated, in comparison to our findings, Ml's effectiveness in decreasing drop-out [33], increasing motivation, and the reduction of pain-related disability $[44,97]$. As far as known no studies are available that evaluated MI's effectiveness on social participation.

In the following, upon several potential reasons and explanations for our findings are elaborated. Those could next to others be related to (1) the uncertainty whether the technique is suitable in patients with non-specific chronic musculoskeletal pain; (2) the low level of Ml-fidelity in this study; (3) the fact that the pre-treatment was not integrated in the subsequent rehabilitation treatment; and (4) the unexpected possibility that the attention control pain education could have caused similar effects as the MI-based pre-treatment. Additionally, upon reasons why dropout was not affected by the pre-treatment is elaborated.

\section{(1) MI does not affect motivation to change deeply rooted cognitions of chronic pain}

$\mathrm{MI}$ is used in different health behaviors and patient groups ranging from substance abuse (e.g. [15]) up to lifestyle modifications (e.g. [49]). The most recent meta-analysis upon MI showed that the reduction of problematic drinking resulted in the biggest effect sizes compared to other health behaviors in the medical setting, and is therefore the most prominent and most opportune target behavior [49].

However, given the complex nature of chronic pain, could it be that this relatively brief add-on pre-treatment with $\mathrm{Ml}$ is not effective in those patients? Underlying chronic pain, a complex behavior can be concealed, with deeply rooted cognitions and beliefs regarding the harmful nature of movements and exercise, which can have a disabling effect. This means also that working on motivation to change those cognitions (e.g. catastrophizing) is a major aim during the pain rehabilitation treatment as its mediating role on treatment outcome is known [82]. The common 
cognitions regarding movements and exercise need to be changed by e.g. reattribution interventions [27]. Furthermore, pain rehabilitation treatment is based on a biopsychosocial perspective. However, cognitions (beliefs) about causes and origin of chronic pain can be different for patients and health care providers [72]. Those divergences in the explanatory model of chronic pain are constantly present [2], and can influence the relationship between patient and health care provider in a negative way [72]. At the same time, a trustful relationship was mentioned as overarching topic of fruitful encounters by both parties, turned out from a systematic review of qualitative studies [72]. In addition, a shared understanding of chronic pain by patients and health care professional was related to a positive pain rehabilitation treatment outcome [69]. Combining those previous findings led to the hypothesis that $\mathrm{MI}$ can serve as promising technique as already was shown for other health behaviors and behavior change. However, in the current Prepare trial MI was not superior as pre-treatment. One explanation could be that the emphasis of motivation for behavior change within other complex health behaviors, such as harmful drinking, lays more on actual behavior change and less on motivation to change deeply rooted cognitions, as it is in chronic pain, which seem far more difficult and takes far more time to change.

Differences in the effectiveness of the pre-treatment were found furthermore depending on type of pain problem. In patients with a distinct pain problem, MI as pre-treatment was superior compared to patients with fibromyalgia in terms of a clinically and statistically significant reduction of pain-related disability (results moderation analysis, see chapter 6). This could explain why indications were found that MI was more effective as add-on to a treatment targeting cognitions regarding chronic pain in the subsequent Graded Exposure pain rehabilitation treatment for patients with a distinct pain problem compared to patients with fibromyalgia.

\section{(2) MI-fidelity was too low to be effective}

Findings of mixed fidelity and Ml-skillfulness (chapter 3 ) and of the process evaluation (chapter 5) could make us conclude that the quality of MI was too low to be effective. For these findings several reasons can be given which are discussed in the following.

The pre-treatment intervention was nurse-led. MI requires a striking change in professional attitude with an accent on abandoning the focus on solving problems. This may feel counterintuitive for some professionals such as nurses who are used to and trained to respond based on their professional expertise. This desire to help people, and to 'fix' their problems is in MI-terms described as the so-called 'righting reflex' [62]. Although performed with best intentions, at the same time, this directive style can work out counter-productive or at least ineffective to help patients to change. The righting reflex avoids people to talk themselves into change which could be a reason for MI's low effectiveness.

The evidence regarding the effectiveness of nurse-led Ml-interventions is indeed mixed. Some studies found MI-skillfulness in nurses providing MI interventions $[25,53,76]$ whereas other studies found mixed or insufficient fidelity in studies using $\mathrm{MI}$ as nurse-led intervention in other 
settings $[9,38,67,91]$. In contrast to this, systematic reviews and meta-analyses on $\mathrm{Ml}$ in medical care settings showed robustness and no different outcome depending on the type of provider $[49,50,68]$. Contrary, two other systematic reviews found indications for increasing effect size depending of an increase in level of professional training [79,94], and this result was confirmed by meta-regression [94]. Unfortunately, in the field of musculoskeletal health and physical health, comparisons are not possible. A systematic review in musculoskeletal health and physical health respectively could not achieve comparability due to heterogeneity between the studies and low trial quality $[18,42]$. To our knowledge, there is no study of nurse-led Ml-interventions in chronic pain rehabilitation treatment available to compare with. Habib et al. (2005) evaluated the effectiveness of two MI-based pre-treatment sessions for patients with chronic pain planning to undergo a rehabilitation treatment [33]. But those who provided the pre-treatment were psychologists instead of nurses. As the protocol of the Prepare intervention was based on the protocol of Habib, duration of the pre-treatment was similar to the Prepare intervention arm. Therefore, this could indicate that psychologists are, due to longer training in general counseling skills, more able than nurses to deliver proper $\mathrm{MI}$ in patients with chronic pain.

To conclude, in the current Prepare trial, nurses were only partially able to provide skillful MI whereas in other areas MI could be learnt by different professions. At least for the current trial in the field of chronic pain, there are indications that nurses had difficulties in learning MI. Explanations regarding the inability of nurses to learn $\mathrm{Ml}$ cannot be found in the existing literature. One potential explanation for the findings could be the combination of the complexity of developing motivation for cognitive change which seems necessary in these patients and is elaborated more upon before, and nurses' difficulty to learn MI. For all areas and professions it seems, that Ml-training needs considerable attention.

Profound time and resources were invested within the Prepare trial in ongoing training and regular inter- and supervision. However, it could be that the Ml-training provided was not sufficient to reach a favorable level of Ml-skills. At the time of the preparation of the intervention, the Ml-training provided was based upon available evidence [46,51,57-59]. Since then, advanced insights became available, e.g. that training and maintaining of Ml-skills in randomized clinical trials (RCTs) could be reached by group feedback [37]. Later on, practical recommendations regarding $\mathrm{Ml}$ training issues are given.

Another aspect which could have led to the low score for MI-fidelity, is the usage of a manual within the Prepare trial. On the one hand, the manual was meant to strive towards an uniformly provided and comparable pre-treatment out of research purposes. On the other hand, the usage of a manual could have had a negative impact on achieving sufficient effect sizes, because the $\mathrm{Ml-counselor} \mathrm{by} \mathrm{trying} \mathrm{to} \mathrm{focus} \mathrm{on} \mathrm{the} \mathrm{treatment} \mathrm{as} \mathrm{mentioned} \mathrm{in} \mathrm{the} \mathrm{manual,} \mathrm{might} \mathrm{not} \mathrm{always}$ have been able to sufficiently focus on the client's needs. This specific feature has been described previously for MI $[45,50]$. During a process evaluation of the Prepare trial, indications were found that the nurses strived to address all elements mentioned in the manual, but reported difficulties 
in performing them properly (see also chapter 5). For example the skill of using complex reflections, a specific technique within $\mathrm{MI}$, was reported as difficult to perform by the nurses. In addition, checks of Ml-integrity complemented this finding (see also chapter 3 ).Therefore, the usage of a treatment manual in the Prepare trial could have influenced Ml's effectiveness negatively.

\section{(3) the pre-treatment was not integrated in the subsequent rehabilitation treatment}

As we were interested in the effectiveness of an Ml-based pre-treatment within the Prepare trial, $\mathrm{MI}$ as counselling strategy was only used in this first phase, during pre-treatment. This implies that the pre-treatment and the actual pain rehabilitation treatment were in terms of counselling characteristics, and in terms of professionals involved, not connected to each other. The pretreatment was embedded in the chain of care for those patients, but the multi-professional team members of the rehabilitation treatment were not trained in the principles of $\mathrm{Ml}$ and the nurses providing the pre-treatment were not part of the rehabilitation treatment team. It could be that this approach undermined MI's effectiveness as an integrated approach hold by all professionals involved would have used Ml's full potential. In contrast to the previous hypothesis, some studies in other fields showed that MI was indeed effective as single short intervention, not included in a holistic treatment approach, and not provided by professionals belonging to the treatment team (e.g.[22]). Furthermore, MI's effectiveness as adjunctive pre-treatment with the aim to enhance motivation and treatment engagement for another treatment is specifically acknowledged in systematic reviews $[13,36,48,94]$. Therefore, the choice to use MI as pre-treatment only seems justifiable.

\section{(4) the pain education attention control pre-treatment could have caused similar effects}

\section{as the MI-based pre-treatment}

Nowadays, extensive research is done regarding the potential of pain education entailing neurophysiological and neurobiological aspects of chronic pain. Education related to these topics seems to have positive effects on pain intensity, pain-related disability, physical performance, attitudes, and cognitions (beliefs) such as catastrophizing (e.g. $[19,47])$. It needs to be mentioned that its level of evidence is yet limited due to low study quality [19]. But in line with the positive effects reported, it could be assumed, that the pain education attention control arm of the Prepare trial could also have been effective and has therefore resulted in non-superiority of the Ml-based intervention arm. The pain education control condition of the Prepare trial consisted of elements such as the discriminative characteristics of acute pain versus chronic pain, which were also addressed in the systematic review [47]. At the same time, the topics discussed in the two Prepare pain education control sessions were based upon older information regarding pain such as pacing and self-management, which seem not to favor pain physiology education (e.g. [55]). Therefore, taking these insights from comparable studies into account, this indicates that this concern is ungrounded, and could therefore not explain the non-superiority of the MI-based pre-treatment intervention compared to the control condition. 


\section{Potential explanations why drop-out from rehabilitation treatment was not affected through pre-treatment}

Another objective of the Prepare trial was to decrease drop-out from treatment and to improve treatment completion by a Ml-based pre-treatment. This was not succeeded with this in the current Prepare trial: The numbers are even comparably high in both pre-treatment arms. Twenty-three percent of the research population dropped out from treatment prematurely. This rate was comparable high with drop-out rates in CBT treatments for patients with chronic low back pain (between 13-30\%) [31]. These findings tell us two things: the numbers of drop-out remain high, and MI could not help to decrease this number. Although one comparable study was indeed able to show that MI could be effective in improving attendance [33], a second was not able to decrease drop-out from pain treatment significantly [41]. In our study, it could be hypothesized that $\mathrm{MI}$ could have fulfilled this role in case it was adequately provided (see chapters 3 and 5). However, based on the current results no conclusions can be drawn whether MI-based pre-treatment could indeed help to decrease drop-out from treatment.

\section{Proposed solutions to decrease drop-out}

The magic bullet to defeat drop-out for rehabilitation treatment is not found yet. It is therefore questionable which approach could help to increase adherence to treatment. From a systematic review it is known that self-efficacy is related to treatment adherence in patients with chronic pain [85]. It is therefore likely that taking into account self-efficacy enhancing components in the treatment could have beneficial effects on treatment outcome and treatment adherence in future studies. It has to be said that self-efficacy was most frequently investigated in the systematic review mentioned.

In addition to this, the role of indication and screening needs to be better investigated. It needs to be determined which approach is more suitable: either to figure out plain criteria for the selection of suitable patients for pain rehabilitation at an initial stage during the process of treatment indication by a consultant in rehabilitation medicine or handling a laissez faire approach till the ultimate indication by the multidisciplinary screening will be done. E.g. indications based upon a sufficient level of a patient's motivation for treatment are conceivable.

Probably, also other solutions are applicable to decrease drop-out. As will be later extensively mentioned, the influential role of expectancy upon outcome of the rehabilitation treatment is important. To explore ways to intervene and modify expectancy could help to decrease drop-out. Another point is the call for more rigorous patient-centered care. It seems that even not directly treatment-related circumstances such as car parking modalities (e.g. [30]) and treatment scheduling options influence the treatment outcome. 


\title{
Measurement instruments for participation and for expectancy
}

\author{
The ICF, the concept of participation, and the USER-P as primary outcome measure \\ The International Classification of Functioning, Disability and Health (ICF) [1] is the main model \\ rehabilitation treatment is founded upon [93]. Within the ICF, participation is defined as "the \\ involvement in life situations" (p. 10) [1]. Activities, another component of the ICF, are defined as \\ "the execution of a task or action by an individual" (p. 10) [1]. Nine chapters are postulated: (1) \\ learning and applying knowledge; (2) general tasks and demands; (3) communication; (4) mobili- \\ ty; (5) self-care; (6) domestic life; (7) interpersonal interactions and relations; (8) major life areas; \\ and (9) community, social, and civic life [1].
}

Due to the importance of participation as outcome measure, the content of the Ml-based pre-treatment was based on the ICF and aimed to enhance social participation after the pain rehabilitation treatment. The chosen primary outcome measure Utrecht Scale for Evaluation of Rehabilitation-Participation (USER-P Participation) is an ICF-based participation measurement instrument [75]. It takes into account chapters 6 through 9 of the ICF which are considered as most characteristic for participation [52,100]. The USER-P is a comprehensive self-report measure of participation that contains subjective as well as objective information regarding participation [90]. This pretty generic instrument was developed to assess outcomes of outpatient rehabilitation treatments [75]. Its psychometric qualities are satisfactory $[75,88,90]$. After our decision to use the USER-P as primary outcome, and after the actual start of the recruitment, it was shown that the USER-P has a low internal consistency [89]. Furthermore, the level of responsiveness appeared to be lower in patients with chronic pain compared to patients with other diagnoses [88]. Therefore, it seems that the USER-P is less suitable for patients undergoing pain rehabilitation. One explanation for the low responsiveness of the USER-P instrument could be that it does not address the fluctuating nature of chronic pain. Looking around, two other ICF-based participation measurement instruments are available. Those are the ICF Measure of Participation and Activities-Screener (IMPACT-S) [74] and the World Health Organization Disability Assessment Schedule II (WHODAS II) [70]. But compared to them, the USER-P is favorable in terms of validity and user-friendliness (in persons with spinal cord injury) [89]. Therefore, even in the light of the above mentioned drawbacks, the choice of the USER-P as primary outcome measure was and is the best available measure at this moment. Furthermore, the need to distinct between subjective and objective aspects of participation is mentioned in other literature $[11,40]$ and the USER-P takes those aspects separately into account.

From our point of view, measuring participation is adequate given the available instruments, but probably a different measure is needed for patients with chronic pain. At present, research taking participation into account is seldom in the Dutch rehabilitation research landscape [93], even although it is the important outcome in rehabilitation treatment. The IMMPACT recommen- 
dations (Initiative on Methods, Measurement and Pain Assessment in Clinical Trials) in chronic pain [24] do not recommend upon the outcome measure social participation. But there is a call to include even more social aspects of the biopsychosocial model in the core set of outcome measurement as those are not well represented there [29]. For the future, ongoing research is definitely required in the field of the measurement of participation in different diagnostic groups. In our group, a project regarding Patient Reported Outcomes Measurement Information System (PROMIS) $[8,16,87]$ of Dutch and Flemish versions of participation measures in different rehabilitation patient populations has started recently. By this, Computer Assisted Testing (CAT) (which is based upon Item Response Theory) could be applied at a later stage for scientific purposes and research purposes. Comparable research regarding pain behavior was already done in a Dutch-Flemish version [21].

Within Prepare, pain-related disability was also measured by the Pain Disability Index (PDI) [17]. The PDI was specifically developed for patients with chronic pain. As the measurement of disability is recommended in the IMPACT recommendations for clinical trials of pain treatment and in the Dutch core set, it was chosen as secondary outcome measure within the Prepare trial. Furthermore, more information regarding its meaningful clinical change is now available [84]. Relying on these insights, clinically meaningful differences could be found favoring the MIbased pre-treatment taking only those patients into account who completed their pre-treatment and their rehabilitation treatment. Furthermore, a moderation analysis showed a significant and clinically relevant decrease in pain-related disability in patients with a localized pain problem compared to patients with fibromyalgia (see also chapter 6).

\section{The measurement of Expectancy}

The important role of treatment expectancy upon treatment outcome is present. From previous research the predictive value of expectancy upon treatment outcome [81] as well as the moderating role of expectancy is known [32]. And it is known, that expectancy is modifiable and should therefore be measured more than once in order to see whether it has changed [92].

There are several measures available to measure treatment credibility and expectancy, but the Credibility and Expectancy (CEO) questionnaire is mostly used although its content validity was currently not known. In the current dissertation its content validity was investigated by an iterative approach (see also chapter 7). As result, a content valid measure of credibility and expectancy is available now. Considering expectancy as a process variable, it is at the moment unknown when exactly expectancy needs to be measured in order to account for the ultimate treatment outcome.

In order to have a standardized and valid method to measure the level of expectancy at multiple times, a second version, the CEQ-2, was developed within the validation process. By using both versions of the CEO, expectancy can be measured during all phases of the treatment in a content valid way. The difference between the CEO and the CEQ-2 is, that the latter one takes into account the changed recall period and time frame after treatment (see chapter 7). But some 
questions regarding expectancy are still open and need future investigation. Some concern the concept, other the construction and course, and the measurement of expectancy. Regarding the concept: Future research is needed for a better understanding of the concept of expectancy and its discrimination from the concept of self-efficacy. Regarding the construction and course, how to best influence, create, and build realistic expectations? And what is the course of expectancy? And how to prevent thereby diverging expectations regarding pain rehabilitation treatment where the main aim is the improvement of functioning rather than pain reduction? Regarding the measurement itself, it is not clear when the best moment at the beginning of the treatment is to measure expectancy.

As general recommendation in the development of suitable measures, qualitative approaches should be an integral part of questionnaire development [73]. Those give a deeper understanding of the respondent's interpretation of the questionnaire.

\section{Implications for clinical practice}

\section{MI-training: Lessons and implications for clinical practice}

While at first glance simple and straightforward principles of $\mathrm{MI}$, those require an empathic and curious attitude. Our research and several others have shown, that "MI is simple but not easy" [61]. And it does seem, that apart from the previously mentioned inconclusive evidence regarding the type of provider [49], it is not for everyone possible to learn MI up to the same level (e.g. $[60,64,66])$. Additionally, not only to learn, but also to sustain Ml-skills is a challenge [28].

Taking into account the aforementioned difficulties in learning and sustaining MI skills, and the findings of our trial (especially chapter 3 and 5), where much effort was put in tailor-made and extensive Ml-training in order to reach proficiency and fidelity, it has to be underlined, that the current practice of 3-day Ml-trainings, as it is provided in several heath care institutions is not an effective way of successful and skillful implementation of MI in daily practice.

Nowadays, four training principles are stated [9]:

(1) Screening and selection;

(2) "one size does not fit all";

(3) "Do more of the same"; and

(4) continuous intervision and supervision.

(1) Screening and pre-selection of potential trainees on beforehand is suggested. Suggestions reach from personality traits or cognitive abilities associated with learning or the motivation to learn (e.g. [58]) up to checks of empathy as this is a probably important predictor for Ml-skillful- 
ness after training completion [66]. In the future, an evaluation should take place regarding the most effective approach. At this moment, no validated way of screening potential candidates for Ml-training is available.

(2) Ml-training approaches should involve as part of the training actual problems encountered by the trainees in clinical practice and taking into account the actual needs of the trainees (e.g. [5]). Furthermore, certain superior training modes, such as actor involvement and peer feedback, seem to be more favorable (e.g. [37]).

(3) Handling a training format based upon continuous feedback by means of objective measures, and adjusted to commonly occurring deviations as recognized by the researcher. This in light of evidence that self-report of trainees in MI regarding skillfulness is not an indicator of the objectively measured Ml-skillfulness [98].

(4) Additionally, the continuous supervision and intervision with others also practicing $\mathrm{MI}$ is needed to sustain MI skills (e.g. $[4,53])$. This could improve perceived social support in the implementation of this approach, as a qualitative study regarding the uptake of MI in clinical practice turned out the feeling insecure and the obstacle of newness can hinder the uptake and usage of $\mathrm{MI}[71]$.

It should be taken into account before the decision to adopt $\mathrm{Ml}$ in clinical practice within an organization, that implementation of $\mathrm{Ml}$ is an enormous investment in terms of time and resources. Furthermore, it requires not only ongoing training in Ml-skills and regular inter-and supervision to prevent the erosion of Ml-skills $[80,83]$, but also organizational characteristics to promote uptake of MI within organizations $[5,101]$.

\section{Methodological considerations}

\section{Study design effect evaluation}

The current Prepare trial was able to report upon relative effectiveness of $\mathrm{MI}$ as pre-treatment. From a methodological point a three-armed RCT with a no-intervention arm would have been able to report upon its absolute effectiveness of $\mathrm{MI}$ as pre-treatment. The usage of an active control group could elucidate the MI-specific working mechanism [78]. By doing so, control for attention would even be possible, which is especially important in behavioral interventions research. As practical constraint, feasibility problems and reaching a sufficient number of study participants need to be mentioned. 
Other than proposed [56], cost-effectiveness and cost-utility data are available, but analyses have not taken place yet. Taking the results of the Prepare trial in mind, it can be questioned whether these analyses are worthwhile to be done. At first, a high level of Ml-fidelity should be assured before taking into account cost-effectiveness and cost-utility.

\section{The research population consisted of different types of chronic pain problems}

The research population of the Prepare trial consisted of a typical sample of different types of chronic pain problems which are characteristic for patients visiting an outpatient department of Rehabilitation Medicine. As we found in the moderation analysis differences in reduction of pain-related disability depending on the type of pain problem (distinct pain problem vs. generalized pain problem) (chapter 6), the comparability of the two types of pain problem could be questioned. The cause of the difference needs further investigation in the future. Therefore, in the future psychosocial and other factors need to be inventoried and compared between the different types of pain problems.

\section{The need for uniform and rigor measurement of MI-fidelity}

The current findings underline the need for a continuous supervision of MI integrity and rigor fidelity evaluations in trials using complex behavioral interventions [6] such as MI throughout a study period. This is also in line with a plea of Miller and Rollnick, the 'fathers' of MI [63]. It seems that within $\mathrm{Ml}$, coding instead of licensing is the chosen way of quality assurance [7]. In this light, it was surprising, that no exact guidance on Ml-fidelity checks was available. In order to do so, an update on current practices and practical recommendations is given in chapter 4 [39]. In line with this, a field guide and a checklist for fidelity of behavioral interventions in general became available now [43]. Additionally, Mars et al. (2013) developed a methodology to assess the level of fidelity within a complex behavior change intervention in chronic pain [54]. Their developmental approach underlines the need for data triangulation and context-specific measures in order to capture the complex construct of intervention fidelity within complex (behavioral) interventions.

\section{The measurement of treatment adherence}

Within Prepare, a rather simple measure of adherence to rehabilitation treatment was used; we assessed whether a patient dropped-out from rehabilitation treatment prematurely. This can be either the consequence of being absent from scheduled treatment sessions more than three times or because patients decided themselves not to continue treatments any longer. At this moment, valid and reliable measures of adherence are lacking in musculoskeletal care [34], and in general [54]. Furthermore, due to regulations to protect patient's rights in study participation, it is not allowed to explore reasons for attrition from the research. Therefore, attendance or frequency measures are often used, but it has to be said that at this moment, no consensus regarding the optimal method for measuring adherence to treatment is reached $[34,85]$. Additionally, seemingly low levels of adherence to treatment or preliminary completion of the treatment, however, may not necessarily be associated with poor outcomes. It can indeed be the result of fast improvement of functioning and decreased pain-related disability throughout the rehabilitation treatment. 


\section{Missing data}

Missing data due to lost-to-follow-up occurred frequently (approximately 55\% at the last follow-up measurement) (chapter 6). Several reasons can be given for this: Patients were pretty eager to receive a pre-treatment in the waiting time between the indication for and the actual start of the rehabilitation treatment, but the time investment to fill in questionnaires even after the decision not to follow a pain rehabilitation treatment was too demanding for some. Some others perceived the self-report questionnaires as intriguing and confronting and preferred not to think about the consequences of their chronic pain. The choice for extensive questionnaires at four follow-up moments could have resulted in "questionnaire fatigue" which could be an explanation of the high level of missing data and even loss to follow up among participants. One could argue that this high amount of missing data due to lost-to-follow-up and drop-out could decrease the external validity of the results. On the other hand, this is the reality of real-life (pragmatic) trials which is confronted by a constant exchange between internal validity and scientific rigor on the one hand, and external validity (the generalizability of the results) on the other hand, which is relevant from a practical and clinical point of view at the same time $[26,86]$.

\section{Strengths and limitations}

\section{This dissertation has strengths and limitations.}

In summary, the usage of different research methods and the incorporation of different perspectives (patients as well as nurses involved, self-report as well as more objective measures by means of audio recording; chapter 3,5, and 6), extensive checks of treatment fidelity in addition to the evaluation of the RCT (chapter 3 ) have enriched the comprehensive understanding of what actually happened within the pre-treatment sessions and how this was received by the patients and nurses involved. By doing so, the explanatory value of the trial was enriched and increased, and complemented our conclusions. This is also in line with key messages of a development of a standard approach for the evaluation of intervention integrity [54]. Strength is the array of perspectives and methodologies applied in this dissertation.

A second strength is the design of randomized controlled trial as used enhancing internal validity. Additionally, several strategies to reduce the risk of bias were applied (e.g. a pre-defined study protocol, treatment manuals, blinding of respondents, and more). However, not all potential and possible biases could be eliminated. At the same time the large heterogeneity of the types of non-specific chronic pain included in the Prepare trial and the embedment in the chain of pain rehabilitation care in the real world are strength and a weakness at the same time. This refers to the already earlier mentioned exchange of internal and external validity [86]. 


\section{Limitations and room for improvement}

Next to strengths there are also limitations and room for improvement. Hindsight, some choices made during the design of the RCT and the delivery of the intervention could have been done better.

During the development of the pre-treatment intervention, we did not strive towards the involvement of patients in the program development. Results of the process evaluation (chapter 5) showed however that a sufficient amount of patients indicated the need for improvements. This was not further explored by means of e.g. focus groups, and therefore cannot be concluded what needs to be changed within the pre-treatment intervention. This was a missed opportunity which should have been given more emphasis already in the development of future programs and (pre-) treatments. Iterative design and co-creation by involving patients in the development of new treatments are relatively new developments within health care and could help to overcome the patients' perceived need for improvement of the pre-treatment $[35,77]$. Examples of recent intervention development show that those approaches are contributing to efficient interventions. At the same time, those are constrained by the extensive need of resources (e.g. [14]). This limits its usage as it involves great investments in terms of financial and human resources. It needs to be mentioned furthermore, that patients as well as researchers need sufficient training to work together on research trials successfully [23].

Probably, the delivery of the intervention could have been improved, in case we would have checked the MI competence of the nurses before the start of the trial. This could have allowed us to act upon the low MI quality already at the beginning. This is also recommended in a recent guideline upon the fidelity of behavioral intervention trials [43].

Furthermore, both pre-treatment conditions were delivered by two different nurses each, which delivered either the Ml-based pre-treatment or pain education. This can have introduced dependence upon person-specific characteristics of those $2 \times 2$ nurses, and allowed less generalizability. Another neglected aspect within this dissertation is, that the focus of theories aiming to explain the working mechanism of MI lies on language content [10]. Linguistic analyses regarding this aspect were not undertaken, but this may be worth in order to understand the working mechanism of $\mathrm{MI}$ more in detail.

\section{Overall conclusion}

To conclude, this dissertation has not confirmed the hypothesis that MI was more effective in terms of social participation, pain-related disability, and the improvement of drop-out from treatment compared to pain education as pre-treatment in patients with chronic pain undergoing pain rehabilitation. In spite of what is often reported about the benefits of MI as pretreatment, in the current context of pain rehabilitation treatment, it has only offered some 
solution to prepare and motivate patients for treatment, to increase social participation, but could not prevent drop-out from treatment. Several reasons are proposed within the different empirical chapters of the current thesis and in this discussion section. However, clinical implications, suggestions for future research directions as well as methodological recommendations for studies in the field of $\mathrm{Ml}$ are given.

\section{Future research directions}

Given the complexity of implementing behavioural interventions, more methodologies need to be developed on how to incorporate and synthesise findings of mixed methods studies in the evaluation of a trial's effectiveness. For example, to date no standards exist how results of a process evaluation will be used in RCT analyses to generalize findings.

The core feature and one important working element within $\mathrm{MI}$ is the so called MI-spirit. This interpersonal interaction style refers to the relational component of $\mathrm{MI}$, containing elements of being supportive regarding a client's autonomy, characterized by a collaborative sphere between client and counselor, and an evocative attitude of the counselor [62]. Beside this, working elements of $\mathrm{MI}$ need to be entangled more in the future. Three reviews regarding of Ml's potential mechanisms of change within the field of addiction treatment [3], within other mental health problems [78], and within health behaviors [20] found consistent evidence for the following constructs: client change talk and the client's experience of discrepancy [3], and MI-spirit and motivation [20], and overall patient engagement [78] is related to better outcomes [20], and MI-inconsistent therapist behavior is related to worse outcomes [3]. In line with the last finding, on the important role of empathy has shed more light as low levels of empathy are regarded as toxic nowadays [66]. It has to be said that quality of the studies included in the review was in general poor. Therefore, we recommend other researchers to study the application of Motivational Interviewing in rigorously designed and comprehensively reported trials. Thereby, more high-quality evidence regarding working mechanisms will become available. At this moment, MI's proficiency level is based on expert opinion. There is surely more research needed to establish its empirical support [65]. More research on working elements would also shed more light upon when $\mathrm{Ml}$ is proficient to be effective. Taking therefore the practical recommendations regarding Ml-fidelity ratings (chapter 4 ) into account in future research projects, this could be helpful in getting an answer on this question in the future.

The aim of the current Prepare trial was to investigate the effectiveness of a pre-treatment. During the moderation analysis it turned out that differences in outcome were depending upon the type of pain problem. As patients with a distinct pain problem received second-wave 
cognitive-behaviorally oriented pain rehabilitation treatment whereas patients with fibromyalgia received third-wave Acceptance-Commitment Therapy (ACT), this let conjecture what the reason for this finding is: the different treatment or the patient group. Anticipating upon an available review this is surprising as ACT is known from a systematic review as an even effective therapy compared to cognitive behavioral therapy (CBT) for patients with chronic pain including fibromyalgia [95]. Future research is definitely needed to investigate this. Therefore, 'what works for whom?' [96] is still an important question to be asked in the field of pain rehabilitation treatment. Probably, this could be the key for more effective treatments in the future. Future research should aim to entangle those factors more in order to allow for the adaptation based upon person-specific factors and requirements. In addition to this, the sufficient duration (dose) of pain rehabilitation treatment and minimally required content is not entangled yet [99]. To entangle dose and content factors is an important direction for future research. 


\section{References}

[1] International Classification of Functioning, Disability, and Health (ICF). ICF full version. Geneva, Switzerland: World Health Organization, 2001.

[2] Allegretti A, Borkan J, Reis S, Griffiths F. Paired interviews of shared experiences around chronic low back pain: classic mismatch between patients and their doctors. Family practice 2010;27(6):676-683.

[3] Apodaca TR, Longabaugh R. Mechanisms of change in motivational interviewing: a review and preliminary evaluation of the evidence. Addiction 2009;104(5):705-715.

[4] Bach S, Holten K. Guideline update: what's the best approach to acute low back pain? Journal of Family Practice 2009;58:E1.

[5] Baer JS, Wells EA, Rosengren DB, Hartzler B, Beadnell B, Dunn C., Agency context and tailored training in technology transfer: a pilot evaluation of motivational interviewing training for community counselors.

Journal of substance abuse treatment 2009;37(2):191-202.

[6] Bellg AJ, Borrelli B, Resnick B, Hecht J, Minicucci DS, Ory M, Ogedegbe G, Orwig D, Ernst D, Czajkowski S., Enhancing treatment fidelity in health behavior change studies: best practices and recommendations from the NIH Behavior Change Consortium. Health Psychology 2004;23(5):443-451.

[7] Björk A., Stabilizing a fluid intervention: The development of Motivational Interviewing, 1983-2013. Addiction Research and Theory 2014;22(4):313-324.

[8] Boers M., Standing on the promises: first wave validation reports of the Patient-Reported Outcome Measurement Information System. Journal of Clinical Epidemiology 2010;63(11):1167-1168.

[9] Bohman B, Forsberg L, Ghaderi A, Rasmussen F., An Evaluation of Training in Motivational Interviewing for Nurses in Child Health Services. Behavioural and Cognitive Psychotherapy 2013;41(03):329-343. 
[10] Bricker J, Tollison S., Comparison of motivational interviewing with acceptance and commitment therapy: a conceptual and clinical review. Behavioural and Cognitive Psychotherapy 2011;39(5):541-559.

[11] Brown M, Dijkers MP, Gordon WA, Ashman T, Charatz H, Cheng Z., Participation objective, participation subjective: a measure of participation combining outsider and insider perspectives. The Journal of head trauma rehabilitation 2004;19(6):459-481.

[12] Burke B, Arkowitz H, Menchola M., The efficacy of motivational interviewing: a meta-analysis of controlled clinical trials. Journal of Consulting and Clinical Psychology 2003;71(5):843-861.

[13] Burke BL, Arkowitz H, Menchola M., The efficacy of motivational interviewing: a meta-analysis of controlled clinical trials. Journal of Consulting and Clinical Psychology 2003;71(5):843-861.

[14] Carnes D, Homer K, Underwood M, Pincus T, Rahman A, Taylor SJC., Pain management for chronic musculoskeletal conditions: the development of an evidence-based and theory-informed pain self-management course. BMJ Open 2013;3(11):e003534.

[15] Carroll KM, Ball SA, Nich C, Martino S, Frankforter TL, Farentinos C, Kunkel LE, Mikulich-Gilbertson SK, Morgenstern J, Obert JL, Polcin D, Snead N, Woody GE. Motivational interviewing to improve treatment engagement and outcome in individuals seeking treatment for substance abuse: a multisite effectiveness study. Drug and Alcohol Dependence 2006;81(3):301-312.

[16] Cella D, Riley W, Stone A, Rothrock N, Reeve B, Yount S, Amtmann D, Bode R, Buysse D, Choi S., The Patient-Reported Outcomes Measurement Information System (PROMIS) developed and tested its first wave of adult self-reported health outcome item banks: 2005-2008. Journal of Clinical Epidemiology 2010;63(11):1179-1194.

[17] Chibnall JT, Tait RC. The Pain Disability Index: factor structure and normative data. Archives of physical medicine and rehabilitation 1994;75(10):1082-1086.

[18] Chilton $R$, Pires Yfantouda R, Wylie M., A systematic review of motivational interviewing within musculoskeletal health. Psychology, Health \& Medicine 2012:17(4):392-407.

[19] Clarke CL, Ryan CG, Martin DJ., Pain neurophysiology education for the management of individuals with chronic low back pain: systematic review and meta-analysis. Manual Therapy 2011;16(6):544-549. 
[20] Copeland L, McNamara R, Kelson M, Simpson S., Mechanisms of change within motivational interviewing in relation to health behaviors outcomes: A systematic review. Patient Education and Counseling 2014;98(4):401-411.

[21] Crins MH, Roorda LD, Smits N, de Vet HC, Westhovens R, Cella D, Cook KF, Revicki D, van Leeuwen J, Boers M, Dekker J, Terwee CB. Calibration of the Dutch-Flemish PROMIS Pain Behavior item bank in patients with chronic pain. European Journal of Pain 2015; doi: 10.1002/ejp.727. [Epub ahead of print].

[22] Cunningham RM, Chermack ST, Zimmerman MA, Shope JT, Bingham CR, Blow FC, Walton MA., Brief motivational interviewing intervention for peer violence and alcohol use in teens: one-year follow-up. Pediatrics 2012;129(6):1083-1090.

[23] Dudley L, Gamble C, Allam A, Bell P, Buck D, Goodare H, Hanley B, Preston J, Walker A, Williamson P, Young B., A little more conversation please? Qualitative study of researchers' and patients' interview accounts of training for patient and public involvement in clinical trials. Trials 2015;16(1):190.

[24] Dworkin R, Turk D, Farrar J, Haythornthwaite J, Jensen M, Katz N, Kerns R, Stucki G, Allen R, Bellamy N, Carr D, Chandler J, Cowan P, Dionne R, Galer B, Hertz S, Jadad A, Kramer L, Manning D, Martin S, McCormick C, McDermott M, McGrath P, Quessy S, Rappaport B, Robbins W, Robinson J, Rothman M, Royal M, Simon L., IMMPACT: core outcome measures for chronic pain clinical trials: IMMPACT recommendations. Pain 2005;113:9-19.

[25] El-Mallakh P, Chlebowy DO, Wall MP, Myers JA, Cloud RN., Promoting nurse interventionist fidelity to motivational interviewing in a diabetes self-care intervention. Research in Nursing and Health 2012;35(3):289-300.

[26] Feilzer MY., Doing Mixed Methods Research Pragmatically: Implications for the Rediscovery of Pragmatism as a Research Paradigm. Journal of Mixed Methods Research 2010;4(1):6-16.

[27] Fink $P$, Rosendal $M$, Toft T., Assessment and treatment of functional disorders in general practice: the extended reattribution and management model--an advanced educational program for nonpsychiatric doctors. Psychosomatics 2002;43(2):93-131.

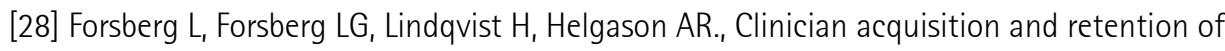
Motivational Interviewing skills: a two-and-a-half-year exploratory study. Substance Abuse Treatment, Prevention, and Policy 2010;5:8. 
[29] Froud R, Patterson S, Eldridge S, Seale C, Pincus T, Rajendran D, Fossum C, Underwood M., A systematic review and meta-synthesis of the impact of low back pain on people's lives. BMC Musculoskelet Disord 2014;15:50.

[30] Gilbert D., Car parking is a clinical quality issue. BMJ 2015;350:h1312.

[31] Glombiewski JA, Hartwich-Tersek J, Rief W. Attrition in Cognitive-behavioral Treatment of Chronic Back Pain. The Clinical Journal of Pain 2010;26(7):593-60.

[32] Goossens ME, Vlaeyen JW, Hidding A, Kole-Snijders A, Evers SM., Treatment expectancy affects the outcome of cognitive-behavioral interventions in chronic pain. The Clinical Journal of Pain 2005;21(1):18-26; discussion 69-72.

[33] Habib S, Morrissey S, Helmes E., Preparing for pain management: a pilot study to enhance engagement. Journal of Pain 2005;6(1):48-54.

[34] Hall AM, Kamper SJ, Hernon M, Hughes K, Kelly G, Lonsdale C, Hurley DA, Ostelo R., Measurement tools for adherence to non-pharmacological self-management treatment for chronic musculoskeletal conditions: a systematic review. Archives of physical medicine and rehabilitation 2014.

[35] Hardyman W, Daunt KL, Kitchener M., Value Co-Creation through Patient Engagement in Health Care: A micro-level approach and research agenda. Public Management Review 2014;17(1):90-107.

[36] Hettema J, Steele J, Miller WR., Motivational interviewing. Annual Review of Clinical Psychology 2005;1:91-111.

[37] Isenhart C, Dieperink E, Thuras P, Fuller B, Stull L, Koets N, Lenox R., Training and maintaining motivational interviewing skills in a clinical trial. Journal of Substance Use 2014;19(1-2):164-170.

[38] Jansink R, Braspenning J, Laurant M, Keizer E, Elwyn G, Weijden Tvd, Grol R., Minimal improvement of nurses' motivational interviewing skills in routine diabetes care one year after training: a cluster randomized trial. BMC Family Practice 2013;14(1):44.

[39] Jelsma JG, Mertens VC, Forsberg L, Forsberg L., How to Measure Motivational Interviewing Fidelity in Randomized Controlled Trials: Practical Recommendations.

Contemporary Clinical Trials 2015;43:93-99. 
[40] Johnston MV, Goverover Y, Dijkers M., Community activities and individuals' satisfaction with them: quality of life in the first year after traumatic brain injury. Archives of Physical Medicine and Rehabilitation 2005;86(4):735-745.

[41] Kerns RD, Burns JW, Shulman M, Jensen MP, Nielson WR, Czlapinski R, Dallas MI, Chatkoff $D$, Sellinger J, Heapy A, Rosenberger P., Can we improve cognitive-behavioral therapy for chronic back pain treatment engagement and adherence? A controlled trial of tailored versus standard therapy. Health Psychology 2014;33(9):938-947.

[42] Knight KM, McGowan L, Dickens C, Bundy C., A systematic review of motivational interviewing in physical health care settings. British Journal of Health Psychology 2006;11(2):319-332.

[43] Knittle K., Fidelity in intervention delivery: A rough field guide. European Health Psychologist 2014;16(5):190-195.

[44] Knittle K, De Gucht V, Hurkmans E, Peeters A, Ronday K, Maes S, Vlieland TV., Targeting motivation and self-regulation to increase physical activity among patients with rheumatoid arthritis: a randomised controlled trial. Clinical Rheumatology $2013 ; 34(2): 231-8$.

[45] Lacker JM, Carosella AM, Feuerstein M., Pain expectancies, pain, and functional self-efficacy expectancies as determinants of disability in patients with chronic low back disorders. Journal of Consulting and Clinical Psychology 1996;64(1):212-220.

[46] Lane $C_{1}$ Hood K, Rollnick S., Teaching motivational interviewing: Using role play is as effective as using simulated patients. Medical Education 2008;42(6):637-644.

[47] Louw A, Diener I, Butler DS, Puentedura EJ., The Effect of Neuroscience Education on Pain, Disability, Anxiety, and Stress in Chronic Musculoskeletal Pain. Archives of Physical Medicine and Rehabilitation 2011;92(12):2041-2056.

[48] Lundahl B, Burke BL., The effectiveness and applicability of motivational interviewing: A practice-friendly review of four meta-analyses. Journal of Clinical Psychology 2009;65(11):1232-1245.

[49] Lundahl B, Moleni T, Burke BL, Butters R, Tollefson D, Butler C, Rollnick S., Motivational interviewing in medical care settings: a systematic review and meta-analysis of randomized controlled trials. Patient Education and Counseling 2013;93(2):157-168. 
[50] Lundahl BW, Kunz C, Brownell C, Tollefson D, Burke BL., A meta-analysis of motivational interviewing: Twenty-five years of empirical studies. Research on Social Work Practice 2010;20(2):137-160.

[51] Madson MB, Loignon AC, Lane C., Training in motivational interviewing: a systematic review. Journal of substance abuse treatment 2009;36(1):101-109.

[52] Magasi S, Post MW., A comparative review of contemporary participation measures' psychometric properties and content coverage. Archives of physical medicine and rehabilitation 2010;91(9 Suppl):S17-28.

[53] Maissi E, Ridge K, Treasure J, Chalder T, Roche S, Bartlett J, Schmidt U, Thomas S, Ismail K., Nurse-led psychological interventions to improve diabetes control: assessing competencies. Patient Education and Counseling 2011;84(2):37-43.

[54] Mars T, Ellard D, Carnes D, Homer K, Underwood M, Taylor SJ., Fidelity in complex behaviour change interventions: a standardised approach to evaluate intervention integrity. BMJ Open 2013;3(11):e003555.

[55] Meeus M, Nijs J, Van Oosterwijck J, Van Alsenoy V, Truijen S., Pain physiology education improves pain beliefs in patients with chronic fatigue syndrome compared with pacing and self-management education: a double-blind randomized controlled trial. Archives of physical medicine and rehabilitation 2010;91(8):1153-1159.

[56] Mertens VC, Goossens ME, Verbunt JA, Koke AJ, Smeets RJ., Effects of nurse-led motivational interviewing of patients with chronic musculoskeletal pain in preparation of rehabilitation treatment (PREPARE) on societal participation, attendance level, and cost-effectiveness: study protocol for a randomized controlled trial. Trials 2013;14(1):90.

[57] Miller W, Mount K., A small study of training in motivational interviewing: does one workshop change clinician and client behavior? Behavioural and Cognitive Psychotherapy 2001;29:457-471.

[58] Miller W, Yahne C, Moyers T, Martinez J, Pirritano M., A randomized trial of methods to help clinicians learn motivational interviewing. Journal of Consulting and Clinical Psychology 2004;72(6):1050- 062.

[59] Miller WR, Moyers TB., Eight stages in learning motivational interviewing. Journal of Teaching in the Addictions 2007;5(1):3-17. 
[60] Miller WR, Moyers TB, Arciniega L, Ernst D, Forcehimes A., Training, supervision and quality monitoring of the COMBINE Study behavioral interventions. Journal of Studies on Alcohol. Supplement. 2005(15):188-195; discussion 168-189.

[61] Miller WR, Rollnick S., Ten things that motivational interviewing is not. Behavioural and Cognitive Psychotherapy 2009;37(2):129-140.

[62] Miller WR, Rollnick S., Motivational interviewing: Helping people change. New York: The Guilford Press, 2013.

[63] Miller WR, Rollnick S., The effectiveness and ineffectiveness of complex behavioral interventions: Impact of treatment fidelity. Contemporary Clinical Trials 2014;37(2):234-241.

[64] Moyers TB, Manuel JK, Wilson PG, Hendrickson SML, Talcott W, Durand P., A Randomized Trial Investigating Training in Motivational Interviewing for Behavioral Health Providers. Behavioural and Cognitive Psychotherapy 2008;36(02):149-162.

[65] Moyers TB, Martin T, Manuel JK, Miller WR, Ernst D., Revised Global Scales: Motivational Interviewing Treatment Integrity 3.1.1 (MITI 3.1.1). In: CoA University of New Mexico, Substance Abuse and Addictions (CASAA) editor, 2010.

[66] Moyers TB, Miller WR., Is low therapist empathy toxic? Psychology of Addictive Behaviors 2013;27(3):878-884.

[67] Noordman J, Van der Lee I, Nielen M, Vlek H, Van der Weijden T, Van Dulmen S., Do trained practice nurses apply motivational interviewing techniques in primary care consultations? Journal of Clinical Medicine Research 2012;4(6):393 - 401.

[68] O'Halloran PD, Blackstock F, Shields N, Holland A, lles R, Kingsley M, Bernhardt J, Lannin N, Morris ME, Taylor NF., Motivational interviewing to increase physical activity in people with chronic health conditions: a systematic review and meta-analysis. Clinical Rehabilitation 2014.

[69] Oosterhof B, Dekker JH, Sloots M, Bartels EA, Dekker J., Success or failure of chronic pain rehabilitation: the importance of good interaction - a qualitative study under patients and professionals. Diasbility and Rehabilitation 2014;15(2):111-118.

[70] World Health Organization. Whodas II disability assessment schedule: Geneva: WHO, 2001. 
[71] Ostlund AS, Wadensten B, Kristofferzon ML, Haggstrom E., Motivational interviewing: Experiences of primary care nurses trained in the method. Nurse Education in Practice 2014;15(2):111-118.

[72] Parsons S, Harding G, Breen A, Foster N, Pincus T, Vogel S, Underwood M., The influence of patients' and primary care practitioners' beliefs and expectations about chronic musculoskeletal pain on the process of care: a systematic review of qualitative studies. The Clinical Journal of Pain 2007;23(1):91-98.

[73] Pool JJ, Hiralal SR, Ostelo RW, van der Veer K, de Vet HC., Added value of qualitative studies in the development of health related patient reported outcomes such as the Pain Coping and Cognition List in patients with sub-acute neck pain. Manual Therapy 2010;15(1):43-47.

[74] Post MW, de Witte LP, Reichrath E, Verdonschot MM, Wijlhuizen GJ, Perenboom RJ., Development and validation of IMPACT-S, an ICF-based questionnaire to measure activities and participation. Journal of Rehabilitation Medicine 2008;40(8):620-627.

[75] Post MW, Van der Zee CH, Hennink J, Schafrat CG, Visser-Meily JM, Van Berlekom SB., Validity of the Utrecht Scale for Evaluation of Rehabilitation-Participation. Disability and Rehabilitation 2012;34(6):478-485.

[76] Robbins LB, Pfeiffer KA, Maier KS, Ladrig SM, Berg Smith SM., Treatment fidelity of motivational interviewing delivered by a school nurse to increase girls' physical activity. The Journal of School Nursing 2012;28(1):70-78.

[77] Robert G, Cornwell J, Locock L, Purushotham A, Sturmey G, Gager M., Patients and staff as codesigners of healthcare services. BMJ 2015;350.

[78] Romano M, Peters L., Evaluating the mechanisms of change in motivational interviewing in the treatment of mental health problems: A review and meta-analysis.

Clinical psychology review 2015;38:1-12.

[79] Rubak S, Sandbaek A, Lauritzen T, Christensen B., Motivational interviewing: a systematic review and meta-analysis. British Journal of General Practice 2005;55(513):305-312.

[80] Schwalbe CS, Oh HY, Zweben A., Sustaining motivational interviewing: a meta-analysis of training studies. Addiction 2014;109(8):1287-1294. 
[81] Smeets RJ, Beelen S, Goossens ME, Schouten EG, Knottnerus JA, Vlaeyen JW., Treatment expectancy and credibility are associated with the outcome of both physical and cognitive-behavioral treatment in chronic low back pain.

The Clinical Journal of Pain 2008;24(4):305-315.

[82] Smeets RJ, Vlaeyen JW, Kester AD, Knottnerus JA., Reduction of pain catastrophizing mediates the outcome of both physical and cognitive-behavioral treatment in chronic low back pain. The Journal of Pain 2006;7(4):261-271.

[83] Söderlund LL, Nilsen P, Kristensson M., Learning motivational interviewing: Exploring primary health care nurses' training and counselling experiences.

Health Education Journal 2008;67(2):102-109.

[84] Soer R, Reneman MF, Vroomen PC, Stegeman P, Coppes MH., Responsiveness and minimal clinically important change of the Pain Disability Index in patients with chronic back pain. Spine 2012;37(8):711-715.

[85] Thompson E, Broadbent J, Bertino MD, Staiger PK., Do Pain-related Beliefs Influence Treatment adherence? A Systematic Review. The Clinical Journal of Pain 2015 [Epub ahead of print].

[86] Thorpe KE, Zwarenstein M, Oxman AD, Treweek S, Furberg CD, Altman DG, Tunis S, Bergel E, Harvey I, Magid DJ, Chalkidou K., A pragmatic-explanatory continuum indicator summary (PRECIS): a tool to help trial designers. Journal of Clinical Epidemiology 2009;62(5):464-475.

[87] Tugwell P, Knottnerus JA, Idzerda L., Tailoring patient reported outcome measurement. Journal of Clinical Epidemiology 2010;63(11):1165-1166.

[88] Van der Zee CH, Kap A, Rambaran Mishre RR, Schouten E, Post MW., Responsiveness of four participation measures for outcomes of outpatient rehabilitation. Journal of Rehabilitation Medicine 2011;43:1003-1009.

[89] van der Zee CH, Post MW, Brinkhof MW, Wagenaar RC., Comparison of the Utrecht Scale for Evaluation of Rehabilitation-Participation with the ICF Measure of Participation and Activities Screener and the WHO Disability Assessment Schedule II in persons with spinal cord injury. Archives of Physical Medicine and Rehabilitation 2014;95(1):87-93. 
[90] van der Zee CH, Priesterbach AR, van der Dussen L, Kap A, Schepers VP, Visser Meily JM, Post MW., Reproducibility of three self-report participation measures: The ICF Measure of Participation and Activities Screener, the Participation Scale, and the Utrecht Scale for Evaluation of Rehabilitation-Participation. Journal of Rehabilitation Medicine 2010;42(8):752-757.

[91] van Eijk-Hustings YJL, Daemen L, Schaper NC, Vrijhoef HJM., Implementation of Motivational Interviewing in a diabetes care management initiative in the Netherlands. Patient Education and Counseling 2011;84(1):10-15.

[92] Van Hartingsveld F, Ostelo RW, Cuijpers P, De Vos R, Riphagen, II, De Vet HC., Treatment-related and patient-related expectations of patients with musculoskeletal disorders: a systematic review of published measurement tools. The Clinical Journal of Pain 2010;26(6):470-488.

[93] van Haster SIG, de Groot IJM, van Meeteren J, Roebroeck ME, Stolwijk-Swüste JM., Inventarisatie Revalidatie-geneeskundig Onderzoek. Nederlands Tijdschrift voor Revalidatiegeneeskunde 2014;4:183-187.

[94] VanBuskirk K, Wetherell J., Motivational interviewing with primary care populations: a systematic review and meta-analysis. Journal of Behavioral Medicine 2013:1-13.

[95] Veehof MM, Oskam M-J, Schreurs KMG, Bohlmeijer ET., Acceptance-based interventions for the treatment of chronic pain: A systematic review and meta-analysis. Pain $2011 ; 152(3): 533-542$.

[96] Vlaeyen JW, Morley S., Cognitive-behavioral treatments for chronic pain: what works for whom? The Clinical Journal of Pain 2005;21(1):1-8.

[97] Vong SK, Cheing GL, Chan F, So EM, Chan CC., Motivational enhancement therapy in addition to physical therapy improves motivational factors and treatment outcomes in people with low back pain: a randomized controlled trial. Archives of Physical Medicine and Rehabilitation 2011;92(2):176-183.

[98] Wain RM, Kutner BA, Smith JL, Carpenter KM, Hu MC, Amrhein PC, Nunes EV., Self-report after randomly assigned supervision does not predict ability to practice Motivational Interviewing. Journal of Substance Abuse Treatment 2015.

[99] Waterschoot FP, Dijkstra PU, Hollak N, de Vries HJ, Geertzen JH, Reneman MF., Dose or content? Effectiveness of pain rehabilitation programs for patients with chronic low back pain: a systematic review. Pain 2014;155(1):179-189. 
[100] Whiteneck G, Dijkers MP., Difficult to measure constructs: conceptual and methodological issues concerning participation and environmental factors. Archive of Physical Medicine and Rehabilitation 2009;90(11 Suppl):S22-35.

[101] Williams J, Blais M, Banks D, Dusablon T, Williams W, Hennessy K., Predictors of the Decision to Adopt Motivational Interviewing in Community Health Settings. The Journal of Behavioral Health Services \& Research 2014;41(3):294-307. 


\section{Summary}

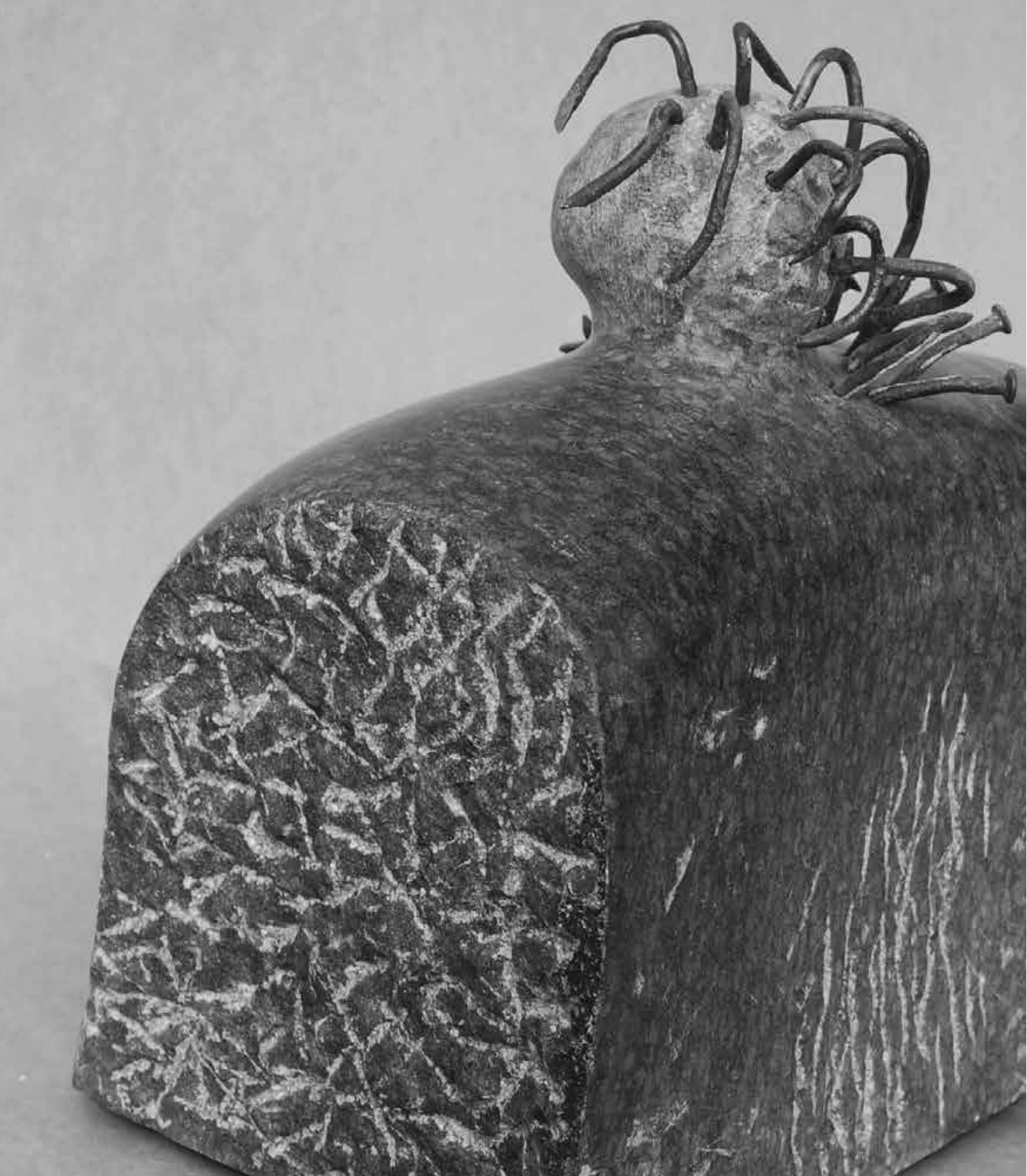




\section{Summary}

Chapter 1 presents a general introduction to the topic of the current dissertation. The background of the current pain rehabilitation treatment and its shortcomings are outlined.

This dissertation presents the results of the research project Pre-Pain Rehabilitation (Prepare). The main aims of this project as presented in this dissertation were:

1. To test the effectiveness of a nurse-led Motivational Interviewing (MI)-based pre-treatment before pain rehabilitation.

2. To test the feasibility, and experiences of patients and nurses involved.

Chapter 2 provides a detailed description of the study protocol of the Pre-pain rehabilitation (Prepare) trial, its rationale, objectives and belonging sub-studies.

Prepare was a single-blind randomized controlled trial. Baseline measurements were administered after randomization to one of the two pre-treatment conditions, with follow-up measurements after the two pre-treatment sessions, at the start of the pain rehabilitation treatment, after finishing the treatment, and at six months follow-up.

The study population comprised of patients with non-specific chronic musculoskeletal pain, who have been indicated for pain rehabilitation treatment by a consultant in rehabilitation medicine. Primary outcome measured was the level of social participation six months after the pain rehabilitation treatment. Secondary outcome measures were the level of pain-related disability and drop-out from pain rehabilitation treatment.

Chapter 3 describes a study aimed at discrimination between both pre-treatment conditions and an evaluation of the Ml-fidelity by means of a validated instrument. Therein, the Motivational Interviewing Treatment Integrity (MITI) instrument was used to score audio recordings of both sorts of pre-treatment, the MI-based pre-treatment sessions and the pain education pre-treatment sessions. This study found, that both conditions could be discriminated. It was also found, that the level of MI-skillfulness was not sufficient in all aspects mentioned in the MITI.

Chapter 4 gives practical recommendations regarding the selection, coding, and reporting of MI fidelity data, as measured by the Motivational Interviewing Treatment Integrity (MITI) code. This is in the light of the urgent need to assess treatment fidelity in order to distinguish between high- and low-quality Motivational interviewing (MI)-interventions. 
Chapter 5 describes the results of a process evaluation, in which the actual delivery and feasibility of the Ml-based pre-treatment intervention was investigated. This process evaluation was performed prior to the effectiveness analysis. The analysis consisted of 1) structured written evaluations of patients involved and 2) structured written registration from the nurses of content, duration and eventual deviations from the treatment manual. Results of this process evaluation indicated that ninety-five percent of the patients who had been allocated to the MI-based pre-treatment intervention group (MIP) participated in the intervention. The intervention was performed according to the pre-specified steps in the treatment manual, but nurses indicated that the quality of their MI performance was below standard. Although the nurses and majority of patients were satisfied with the program, they also identified some limitations. To improve the feasibility, recommendations for improvement are given in this chapter.

Chapter 6 presents the effectiveness of the Prepare study. Prepare was a randomized controlled trial (RCT) wherein 163 patients with non-specific chronic musculoskeletal pain were included. In this study two sessions of either a nurse-led Motivational Interviewing based pre-treatment (MIP) or a nurse-led pain education were provided to the randomly allocated patients. The study showed that results were not statistically significant different between both conditions in terms of participation, pain-related disability, and drop-out from treatment in the intention-to-treat analyses. Per-protocol analysis taking into account only those patients who completed their pre-treatment and rehabilitation treatment showed a larger decrease in related disability, which resulted in a statistically significant difference between both groups favoring MIP. Moderation analysis showed that MIP is effective for improving participation and decreasing pain-related disability in patients with a localized pain problem but not in patients with fibromyalgia. More research is needed into the effectiveness for patients with chronic pain and into Ml skills training, especially in nurse-led care.

Chapter 7 describes the content validation of the Credibility and Expectancy Questionnaire (CEO) as treatment expectancy is important for the ultimate treatment outcome. Therefore, cognitive interviews took place in a sample of 17 chronic pain patients in different phases of their treatment. Chronic pain patients waiting for rehabilitation treatment interpreted and responded to the CEO as intended. Through subsequent adaptations of the CEO in 5 rounds, the instruction text of the CEO was changed. For patients who had already undergone rehabilitation treatment, interpretation and response problems continued. After considerable improvement by adapting several references and time frames, the CEO proved to be a content-valid questionnaire.

Chapter $\mathbf{8}$ is the general discussion in which the main findings of the current dissertation are discussed and reflected upon. To conclude, this dissertation has not confirmed the hypothesis that MI was more effective in terms of social participation, pain-related disability, and the improvement of drop-out from treatment compared to pain education as pre-treatment in patients with chronic pain undergoing pain rehabilitation. In spite of what is often reported about the benefits of $\mathrm{MI}$ as pre-treatment, in the current context of pain rehabilitation treatment, it has only offered 
some solution to prepare and motivate patients for treatment, to increase social participation, but could not prevent drop-out from treatment. Several reasons are proposed within the different empirical chapters of the current thesis and in the discussion section. However, clinical implications, suggestions for future research directions as well as methodological recommendations for studies in the field of $\mathrm{Ml}$ are given.

\section{Samenvatting}

Hoofdstuk 1 is een algemene inleiding van dit proefschrift. Het beschrijft allereerst de achtergrond van pijnrevalidatie in Nederland en op welke gebieden er verbetering nodig is.

Vervolgens introduceert hoofdstuk 1 het onderzoeksproject 'Pre-pain rehabilitation' (afgekort als Prepare), gericht op het voortraject bij pijnrevalidatie van mensen met aspecifieke chronische musculoskeletale pijn. Verpleegkundigen zijn getraind om in het traject voorafgaand aan pijnrevalidatie twee van elkaar verschillende soorten van een voorbehandeling toe te passen. Of motiverende gespreksvoering (MGV) (Motivational Interviewing (MI) of pijneducatie.

De doelstellingen van de studie zijn:

1. het onderzoeken van de effectiviteit van de twee soorten voorbehandelingen tijdens het voortraject bij pijnrevalidatie (effectstudie)

2. het evalueren van de haalbaarheid van de interventie en de ervaringen van de patiënten en verpleegkundigen met het voortraject bij pijnrevalidatie (procesevaluatie).

Hoofdstuk 2 geeft een gedetailleerde beschrijving van het onderzoeksprotocol van de Prepare-studie. Het hoofdstuk beschrijft de achtergrond en doelstellingen van de trial en de bijbehorende deelstudies. De Prepare-studie betreft een enkelblind gerandomiseerde en gecontroleerde trial. De onderzoekspopulatie bestaat uit patiënten met aspecifieke chronische musculoskeletale pijn, die door een revalidatiearts een indicatie hadden gekregen voor een pijnrevalidatiebehandeling. Deze onderzoeksdeelnemers ontvingen voorafgaand aan de pijnrevalidatie twee sessies, aangeboden door verpleegkundigen. De verpleegkundigen uit de interventiegroep waren getraind in het toepassen van de technieken van motivational interviewing.

De nulmeting vond plaats na randomisatie van de onderzoeksdeelnemers. De eerste vervolgmeting werd gedaan na de twee sessies van het voortraject en aan het begin van de revalidatiebehandeling. Na het beëindigen van de revalidatiebehandeling vond opnieuw een vervolgmeting plaats. De laatste meting was zes maanden na het beëindigen van de revalidatiebehandeling. De primaire uitkomstmaat was sociale participatie zoals gemeten zes maanden na het beëindigen van de revalidatiebehandeling. De secundaire uitkomstmaten waren de mate van pijn-gerelateerde beperkingen en uitval uit de pijnrevalidatiebehandeling. 
Hoofdstuk 3 beschrijft een studie naar het onderscheid tussen het voortraject waarbij motivational interviewing werd toegepast door de verpleegkundigen, en het voortraject waar pijneducatie werd toegepast. Op basis van een gevalideerd instrument, het "Motivational Interviewing Treatment Integrity (MITI) instrument", zijn de kwaliteit en integriteit van het voortraject geëvalueerd. De MITI is gebruikt bij het beoordelen van audiotapes van de twee type voortrajecten. De resultaten in hoofdstuk 3 laten zien dat het instrument in staat is om de twee type trajecten van elkaar te onderscheiden. Verder blijkt uit de studie dat het niveau van vaardigheden in motiverende gespreksvoering van de verpleegkundigen niet voor alle gemeten aspecten van MI voldoende was.

Voor het beoordelen van de kwaliteit en integriteit van motiverende gespreksvoering, biedt hoofdstuk 4 een aantal praktische adviezen omtrent de selectie, het coderen en het rapporteren van de kwaliteit en integriteit van interventies waarin motiverende gespreksvoering wordt gebruikt. Deze adviezen zijn gebaseerd op literatuuronderzoek en een verzameling van best practices.

Hoofdstuk 5 beschrijft de resultaten van de procesevaluatie. Het hoofdstuk geeft inzicht in de mate waarin de interventie is toegepast, en evalueert de haalbaarheid van een op motiverende gespreksvoering gebaseerd voortraject. De procesevaluatie is uitgevoerd voorafgaand aan de effectevaluatie. De evaluatie bestond uit een analyse van 1) de evaluatieformulieren die door patiënten ingevuld zijn en 2) evaluatieformulieren die door de verpleegkundigen ingevuld zijn. De vragenlijsten bevatten vragen over de inhoud, de tijdsduur en in hoeverre er is afgeweken van het behandelingsprotocol. De resultaten laten zien dat 95\% van alle patiënten die toegewezen waren aan het op Ml-gebaseerde voortraject ook daadwerkelijk deelnamen. De interventie werd uitgevoerd volgens het protocol. De verpleegkundigen en de meeste patiënten waren tevreden over het voortraject. Er werden ook tekortkomingen geconstateerd. De verpleegkundigen vonden dat de kwaliteit van hun MI-technieken niet voldeden. Het hoofdstuk beschrijft ten slotte adviezen voor verbetering van de interventie.

Hoofdstuk 6 beschrijft de effecten van het Prepare-trial. In totaal 163 patiënten met aspecifieke chronische musculoskeletale pijn hebben deelgenomen aan het gerandomiseerde onderzoek. Het voortraject bestond uit twee sessies met een verpleegkundige. De patiënten die per toeval aan de ene groep waren toegewezen kregen een op motiverende gespreksvoering gebaseerde voorbehandeling, de andere groep patiënten ontving pijneducatie. Op basis van een intention-to-treat analyse zijn geen statistisch significante verschillen gevonden tussen de twee groepen ten aanzien van participatie, pijn-gerelateerde beperkingen, en uitval uit de behandeling. Een zogenoemde per-protocol analyse, waarin alleen patiënten worden meegenomen die zowel de voorbehandeling als de revalidatiebehandeling hebben gevolgd, lieten een sterkere verlaging zien van de mate van pijn-gerelateerde beperkingen bij de deelnemers die de MI-sessies kregen aangeboden. Verder blijkt uit de moderatie analyse dat het MI-voortraject bij patiënten met gelokaliseerde pijnklachten effectiever is dan pijneducatie voor het verbeteren van sociale participatie en het 
verlagen van pijn-gerelateerde beperkingen. Dit geldt echter niet voor patiënten met fibromyalgie. $0 p$ basis van de resultaten wordt geconcludeerd dat er meer onderzoek nodig is naar de effectiviteit van motiverende gespreksvoering bij patiënten met chronische pijn. Hetzelfde geldt voor onderzoek naar training in motiverende gespreksvoering, met name als dit wordt uitgevoerd door verpleegkundigen.

Hoofdstuk 7 beschrijft de inhoudsvalidatie van de zogenaamde 'Credibility and Expectancy Questionnaire (CEQ)" -vragenlijst. Uit onderzoek blijkt dat verwachtingen over de uitkomst van de behandeling belangrijke parameters zijn voor de uitkomst van de behandeling. Met 17 patiënten met chronische pijn zijn cognitieve interviews gehouden. Deze patiënten bevonden zich in verschillende fases van hun pijnrevalidatiebehandeling. Patiënten op de wachtlijst voor een pijnrevalidatiebehandeling interpreteerden en beantwoordden de vragenlijst zoals beoogd. In vijf opeenvolgende rondes is de instructietekst van de CEO aangepast. Patiënten die al aan de behandeling waren begonnen bleven problemen ervaren met de interpretatie en het beantwoorden van de vragenlijst. Op basis van het proces konden verbeteringen worden aangebracht, waardoor de aangepaste CEO kan worden beschouwd als een inhoudsvalide vragenlijst.

Hoofdstuk 8 vormt de overkoepelende discussie van dit proefschrift. Het reflecteert op de hoofdbevindingen uit de Prepare-studie. Ondanks dat de literatuur de potentiele opbrengsten van motiverende gespreksvoering schetst, kon dit niet bevestigd worden in de Prepare-studie. In vergelijking met pijneducatie als voorbehandeling voor pijnrevalidatie werkte motiverende gespreksvoering niet significant beter ten aanzien van sociale participatie, pijn-gerelateerde beperkingen, en uitval uit de behandeling van patiënten. Motiverende gespreksvoering in de context van pijnrevalidatie is een optie om patiënten op hun pijnrevalidatie voor te bereiden en te motiveren voor hun behandeling, en hun sociale participatie te vergroten. Het kon de uitval uit de behandeling echter niet verminderen. Hier kunnen verschillende redenen voor worden genoemd, die in hoofdstuk 8 worden beschreven. Het hoofdstuk sluit af met klinische implicaties, suggesties voor verder toekomstig onderzoek en methodologische adviezen voor onderzoek in het veld van motiverende gespreksvoering. 


\section{Zusammenfassung}

Die vorliegende Dissertation präsentiert die Resultate des Forschungsprojektes 'Pre-pain rehabilitation' (Prepare). In dieser Studie wurde eine Vorbehandlung vor dem eigentlichen Beginn der Schmerzrehabilitationsbehandlung untersucht.

Im Folgenden wird diese als Vorbehandlung oder Prepare Studie bezeichnet werden.

Kapitel 1 gibt eine (allgemeine) Einleitung in das Thema. Hintergründe und Besonderheiten der Schmerzrehabilitation in den Niederlanden werden beschrieben sowie zugehörige Probleme erörtert.

Dieses Projekt hatte folgende Ziele:

1. Den Effekt einer Vorbehandlung vor der eigentlichen Schmerzrehabilitation zu untersuchen, die auf Prinzipien der motivierenden Gesprächsführung basiert.

2. Die Anwendbarkeit der Methode zu testen sowie die Meinungen über und Erfahrungen des Pflegepersonals und der Behandelten in Bezug auf Prepare zu untersuchen.

Kapitel 2 beschreibt detalliert den Studienaufbau der Prepare Studie und weiterer Teilstudien. In der Prepare Studie wurden zwei unterschiedliche Arten einer Vorbehandlung untersucht. Die Teilnehmerlnnen wurden hierzu nach dem Zufallsprinzip (Randomisation) für eine der zwei Arten der Vorbehandlung eingeteilt. Entweder bestand die Vorbehandlungs aus

(1) zwei Terminen bei denen die Prinzipien der motivierenderen Gespächsführung angewandt wurden, oder

(2) zwei Terminen in denen mehr Informationen und Aufklärung über die Besonderheiten von chronischen Schmerzen, den Folgen und möglichen Behandlungsstrategien während der Schmerzrehabilitation gegeben wurden (Schmerzedukation).

Ziel dieser Vorbehandlung war es, die langfristige Teilhabe am sozialen Leben zu verbessern und durch Schmerzen verursachte Beeinträchtigungen zu lindern.

Desweiteren wurde untersucht, ob eine der beiden Vorbehandlungen besser geeignet ist, um einem vorzeitigen Abbruch der Schmerzrehabilitation durch die PatientInnen vorzubeugen.

Zu fünf Zeitpunkten innerhalb eines Jahres wurden die Teilnehmerlnnen gebeten Fragebögen auszufüllen: nach der Einteilung in eine der zwei Gruppen, nach der Vorbehandlung, zu Beginn der Schmerzrehabilitation, nach dem Ende der Schmerzrehabilitation (Dauer ungefähr 12 Wochen ambulant), und abschließend sechs Monate nach dem Ende der übergeordneten Behandlung.

TeilnehmerInnen an der Prepare Studie waren Menschen mit unspezifischen chronischen muskuloskelettalen Schmerzen (Schmerzen im Stütz- und Bewegungsapparat), die durch einen Rehabilitationsmediziner für eine Schmerzrehabilitation angemeldet wurden.

Kapitel 3 beschreibt eine Teil-Studie, die untersucht, inwieweit die beiden Vorbehandlungen voneinander unterschieden werden können, und was deren Qualität ist. 
Dafür wurde ein Instrument eingesetzt, dass speziell für die motivierende Gesprächsführung entwickelt wurde. Dieses Instrument wird als Motivational Interviewing Treatment Integrity (MITI) Instrument bezeichnet.

Dabei werden Audioaufnahmen der Gespräche der Prepare Vorbehandlung analysiert und nach speziellen Kriterien, die in der motivierenden Gesprächsführung als wichtig erachtet werden, beurteilt.

Bei dieser Beurteilung konnten die beiden Arten von Vorbehandlung voneinander unterschieden werden. Desweiteren zeigte sich, dass die Qualität der motivierenden Gesprächsführung nicht allen Qualitätskriterien des MITI-Instrumentes entsprach.

Kapitel 4 gibt praktische Empfehlungen, in welcher Form die Qualität von motivierender Gesprächsführung mit dem MITI Instrument am Besten beurteilt werden kann.

Dies erfolgt aus der dringenden Notwendigkeit heraus, die Qualität von Interventionen motivierender Gesprächsführung beurteilen zu können.

Kapitel 5 beschreibt die Resultate der Prozessevaluation. Darin wurde die Durchführung und die Anwendbarkeit einer auf den Prinzipien der motivierenden Gesprächsführung basierenden Intervention untersucht. Diese Prozessevaluation wurde vor der Effektevaluation durchgeführt. Die Evaluation bestand aus 1) strukturierten Fragebögen für die Teilnehmerlnnen, und 2) strukturierten Fragebögen für das beteiligte Pflegepersonal.

Fünfundneunzig Prozent der TeilnehmerInnen die für eine der beiden Arten der Vorbehandlungen in der Prepare Studie eingeteilt wurde, hat auch tatsächlich daran teilgenommen. Die Intervention wurde wie im Behandlungsplan vorgesehen durchgeführt. Gleichzeitig gab das Pflegepersonal aber auch an, dass sie die Qualität der motivierenden Gesprächsführung niedriger einschätzten als der durchschnittliche Standard. Obwohl die meisten Teilnehmerlnnen und das Pflegepersonal angaben, dass sie mit den Vorbehandlungen innerhalb der Prepare Studie zufrieden waren, gaben sie auch Verbesserungsmöglichkeiten an. In diesem Kapitel werden Empfehlungen zur Verbesserung der Praktikabilität gegeben.

Kapitel 6 beschreibt die Effektivität der Prepare Studie. Prepare war eine randomisierte und kontrollierte Studie (RCT).

Insgesamt wurden 163 PatientInnen mit unspezifischen chronischen muskuloskelettalen Schmerzen inkludiert.

Die Studie zeigte keine statistisch signifikanten Unterschiede zwischen den beiden Arten der Vorbehandlung in Bezug auf soziale Teilhabe, durch Schmerzen verursachte Beeinträchtigungen und dem frühzeitigen Abbrechen der Schmerzrehabilitation.

Bei einer Analyse, die nur die Teilnehmerlnnen einbezog die auch wirklich die Vorbehandlung und die Schmerzrehabilitation abgeschlossen hatten, konnte ein stärkerer Rückgang von durch Schmerzen verursachten Beeinträchtigungen gezeigt werden. Daraus resultiert ein statistisch signifikanter Unterschied mit einem günstigeren Effekt der auf motivierender Gesprächsführung basierenden Vorbehandlung. 
Desweiteren wurde eine Analyse möglicher Moderatoren durchgeführt. Moderatoren sind Faktoren, die die Kausalität beeinflussen. Die Analyse zeigte, dass eine auf motivierender Gesprächsführung basierende Vorbehandlung bei PatientInnen mit einem lokalisierten Schmerzproblem effektiver die Teilhabe vergrößert und durch Schmerzen verursachte Beeinträchtigungen vermindert. Im Gegenzug gilt dies nicht für PatientInnen mit der Diagnose Fibromyalgie (Weichteilrheumatismus).

Es sind weitere Untersuchungen notwendig um die Effektivität von motivierender Gesprächsführung bei PatientInnen mit chronischen Schmerzen zu untersuchen. Ebenso ergeben sich weitere Forschungsnotwendigkeiten im Bezug auf ideale Vermittlungs-Methoden der Prinzipien von motivierender Gesprächsführung.

Kapitel 7 beschreibt eine Validierungsstudie eines verwendeten Fragebogens der Glaubwürdigkeit und Erwartungen misst (Credibility and Expectancy Questionnaire (CEO)). Es ist bekannt, dass beides für das Behandlungsresultat wichtig ist.

Dafür wurden Interviews mit 17 PatientInnen mit chronischen Schmerzen in verschiedenen Behandlungsphasen durchgeführt.

PatientInnen, die auf den Beginn der Schmerzrehabilitation warteten, interpretieren und beantworteten den CEO wie von den EntwicklerInnen beabsichtigt.

Nach Anpassung des instruktiven Textes in fünf Schritten war der Fragebogen gut zu verstehen. PatientInnen die ihre Schmerzrehabilitation bereits abgeschlossen hatten, hatten Probleme den Fragebogen so zu interpretieren und zu beantworten wie von den Entwicklerlnnen intendiert. Nach erheblichen Anpassungen der Vergleichsmodi und des Zeitrahmens dieser Vergleiche erwies sich der CEO als inhaltsvalider Fragebogen.

Kapitel 8 ist die allgemeine Diskussion dieser Dissertation, darin werden die wichtigsten Ergebnisse diskutiert und reflektiert. Die motivierende Gesprächsführung zeigt vielversprechende Ergebnisse in verschiedenen Untersuchungsumgebungen. Im Rahmen der Schmerzrehabilitation zeigt sie sich nur bedingt als Lösung um PatientInnen auf ihre Behandlung vorzubereiten, zu motivieren und ihre langfristige soziale Teilhabe zu verbessern.

Zusammenfassend konnte diese Dissertation daher nicht die Hypothese verifizieren (beweisen), dass motivierende Gesprächsführung im Vergleich zu Schmerzedukation effektiver ist um die soziale Teilhabe zu vergrößern oder durch Schmerzen verursache Beeinträchtigungen und den vorzeitigen Abbruch der Schmerzrehabilitaton von Menschen mit chronischen Schmerzen zu verkleinern.

Abschließend werden klinische Implikationen, Anregungen und methodologische Empfehlungen für zukünftige Foschungsvorhaben im Bereich der motivierenden Gesprächsführung gegeben. 


\section{Acknowledgements Dankwoord Dankwort}

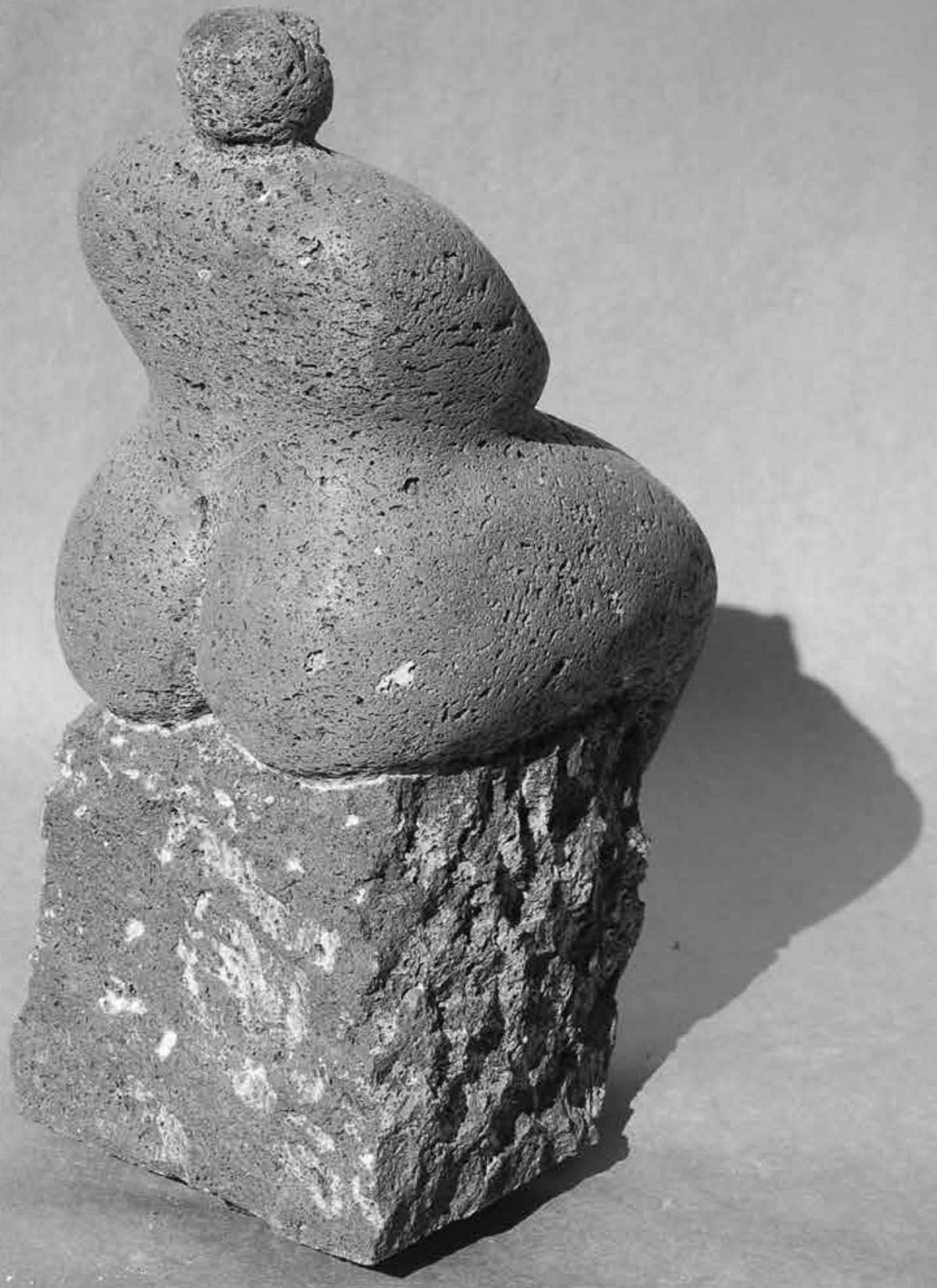




\section{Acknowledgements - Dankwoord - Dankwort}

Wat hebben wandeltochten in de bergen, goede films, en promoveren met elkaar gemeen? Je doet het niet alleen. Gelukkig maar.

Daarom wil ik graag iedereen bedanken die direct en indirect heeft bijgedragen aan de totstandkoming van dit proefschrift. Een aantal mensen wil ik graag uitdrukkelijk bedanken.

\section{Hoofdrol \\ 163 patiënten met chronisch pijn die bereid waren aan het Prepare voortraject \\ deel te nemen}

Prepare verpleegkundigen

Barbara Engelen

Harrie Boetsen ( $t)$

Marc Op den Camp

Simonetta van Ool

\section{Onderzoeksassistentes}

Kitty van der Meer

Astrid Dello

De complemementaire moeders (begeleidsters)

Marielle Goossens

Jeanine Verbunt

\section{Promotor}

Rob Smeets

\section{Werven van patiënten}

Alle revalidatieartsen van het azM en het Atrium

\section{Planning}

Hans Veders

Tammy Postema

Brenda Meijs 
Online vragenlijsten

Marga van der Aa

Layout Prepare Logo

Verena Kaluza

MI-training

Wilma Noteborn

An Geenen

Student assistentes/ WESP studentes

Sanne Pulles

Chantal Snels

Kris Philippi

Collega's

Vakgroep revalidatiegeneeskunde UM

Adelante kenniscentrum

Afdeling revalidatiegeneeskunde azM

Sociale ondersteuning, dagelijkse fika

Alle kamergenoten in Hoensbroek en Maastricht

Hospitality and science-related fika

All coders from Mic-lab

MITI coding zelfhulpgroep

Judith Jelsma

Weekly cinema club ${ }^{+}$

Jelena

Paranimfen cq. trouwgetuigen cq. Trauzeuginnen

Anneke van Dijk

Josephine Jackisch

Nicht-wissenschaftliches Interesse an meiner Person, Wochenenden, Telefonate, Karten Freundinnen und Freunde

Gezelligheid, wandelingen, bezoekjes, lieve kaartjes

Vrienden en vriendinnen 
Mo und Mi 19h: Therapeutisches Laufen

Sandra

Weekendjes, reizen naar vage landen en ander vermaak

Scouting zonder kerk annex Spring

\section{Meinen Eltern}

Katrin und Dieter

Meine Geschwister

Anja

Hanna

Claudius

Cornelius

Immerwährender weiser Rat

Pütti

Die weitere Verwandtschaft

Oma und Omi,

Tanten und Onkel,

Cousinen und Cousins

Mijn schoonfamilie

Annelies en Flip

Yvette en Martijn, Hazel en Tibbe

\section{Zweitfamilie}

Meike, Maren und Charlotte

Co-familie

Karolien en Helder

\section{Treue Freundschaft}

Karin

Geduld met mijn ongeduld, onvoorwaardelijke steun en vermaak Steven 


\section{About the Author}

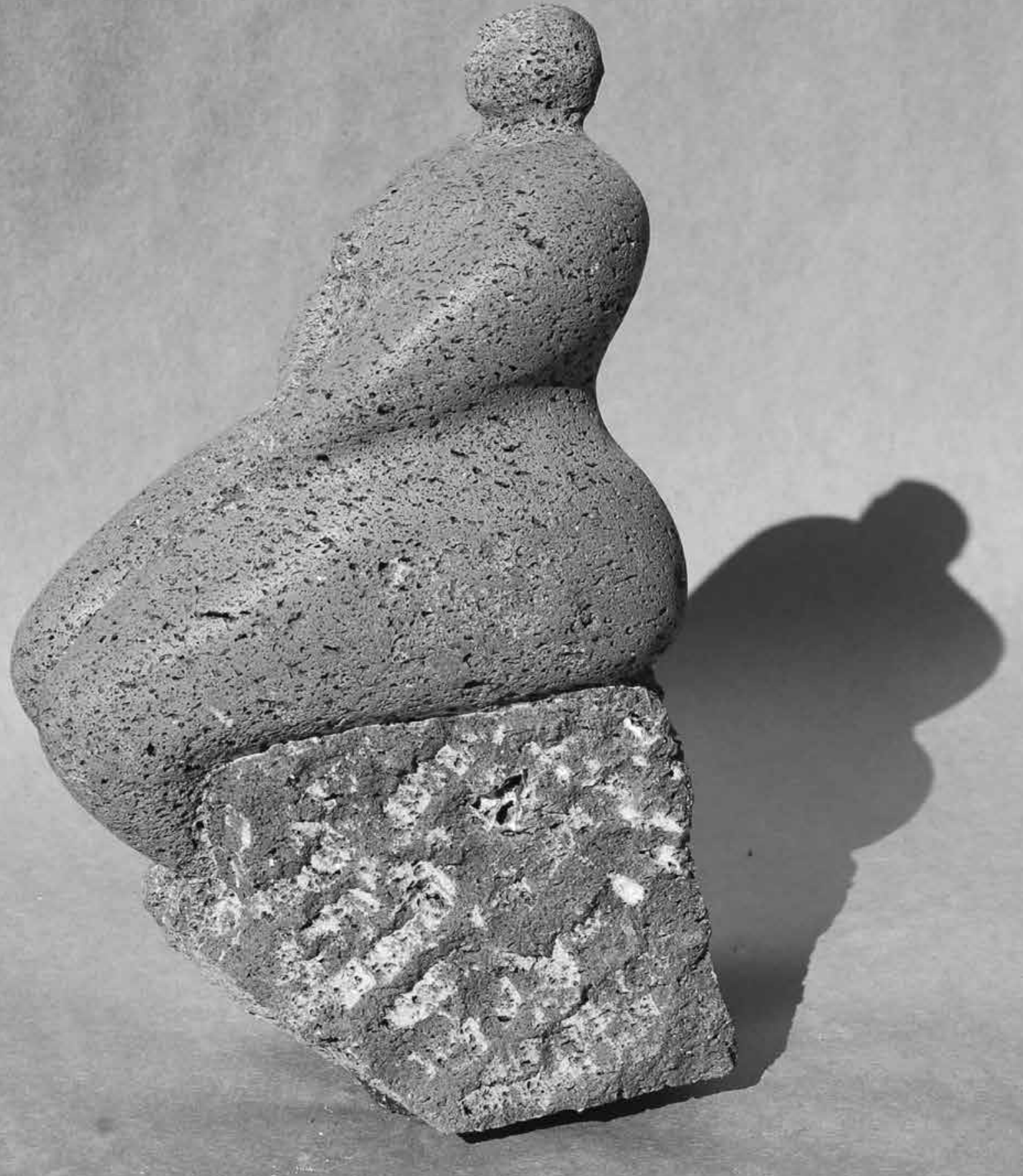




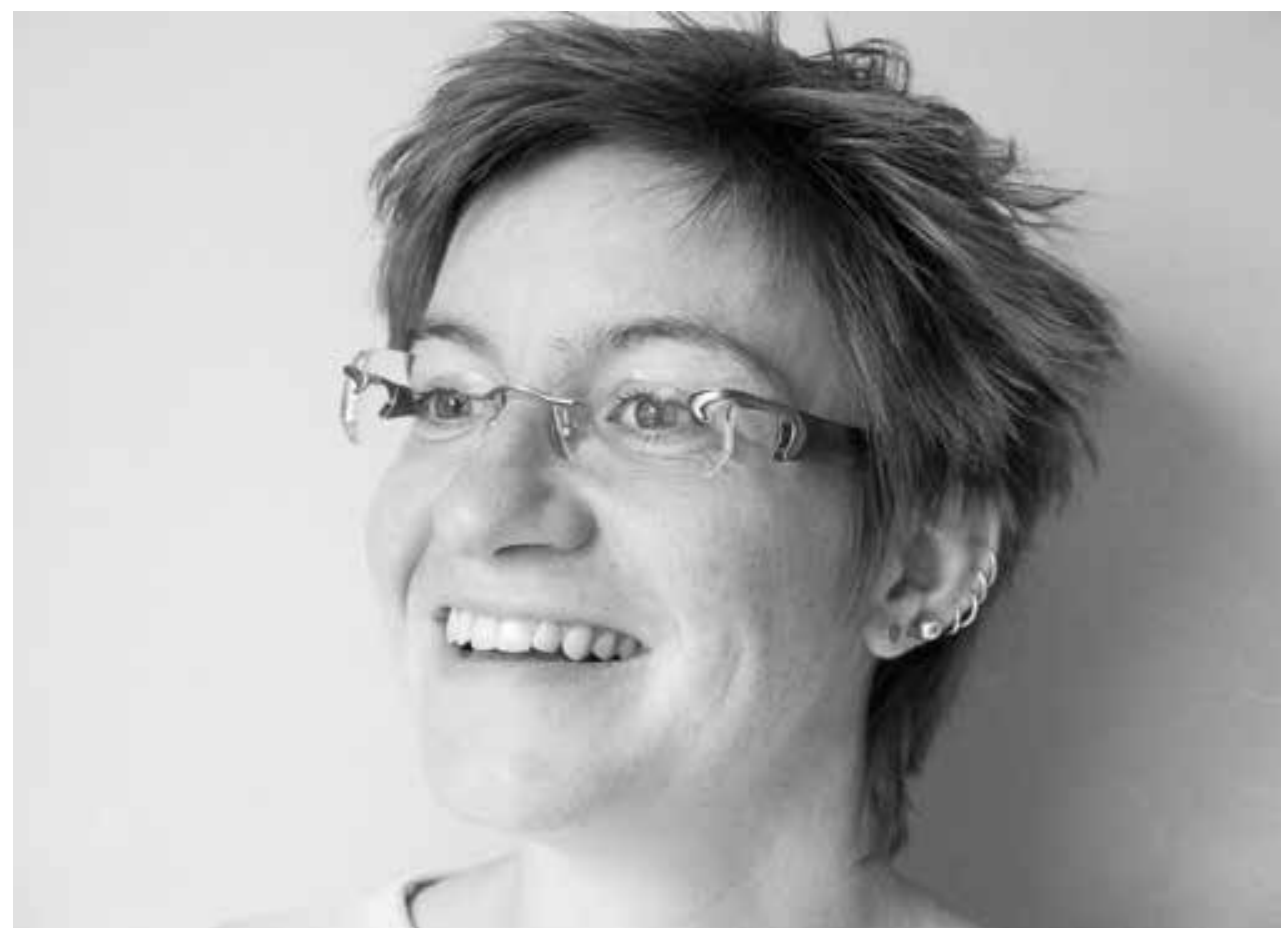

Vera-Christina Mertens 


\section{Curriculum Vitae}

\section{Vera-Christina Mertens}

Vera-Christina was born on 7 July 1982 in Bad Kreuznach (Germany) as the oldest of five children of Katrin and Dieter Mertens (geb. Dotzauer).

After secondary school (Abitur), she did a vocational training in acute ambulance care. After becoming interested in how people live with chronic illness, she studied Rehabilitation Psychology (Magdeburg-Stendal University of Applied Sciences, Germany) and General Health Sciences (Maastricht University, Netherlands and Mälardalen University College, Sweden).

She became intrigued by clinical research while working as a student assistant for the ALASCA trial, a research project on cardiac arrest survivors. During this time, she also gained insight into Dutch culture by visiting patients at their homes in villages in Limburg.

The Health Sciences Research master further enabled her to propose, plan and conduct research in health sciences and healthcare. Vera-Christina majored in Epidemiology and Health Technology Assessment. Her internship, at the department of General Practice/Social Medicine, was in a project to support the self-management of the chronically ill.

After graduation in 2010, Vera-Christina worked as a PhD candidate for the next five years at the Department of Rehabilitation Medicine at Maastricht University, the Netherlands. For her doctoral studies, she conducted an RCT about the effectiveness, fidelity, and feasibility of Motivational Interviewing in patients with chronic pain.

Between the academic years of 2010 and 2012, Vera-Christina was a member of the Faculty Council of the Faculty of Health, Medicine, and Life Sciences at Maastricht University.

Vera-Christina obtained a travel grant from the Dutch Arthritis Foundation (reumafonds) and was a visiting researcher at the Karolinska Institutet MIC lab (leader: Lars Forsberg, PhD) from September till December 2013.

Since September 2015 she has been a Research Associate at the University of Luxembourg where she is investigating interoception in patients with somatoform disorders.

In her private life, Vera-Christina is a volunteer at Nivon Spring (www.springreizen.nl), the Dutch Friends of Nature organization for people between 25 and 40. 


\section{List of publications and presentations}

\section{Peer-reviewed publications}

Vera-Christina Mertens; Lars Forsberg; Jeanine Verbunt; Rob Smeets; Mariëlle Goossens. Treatment fidelity of a nurse-led Motivational interviewing-based pre-treatment in pain rehabilitation. Behavioral Health Services \&t Research 2015.

Judith Jelsma*; Vera-Christina Mertens* (shared 1st authorship); Lisa Forsberg, Lars Forsberg.

How to measure motivational interviewing fidelity in randomized controlled trials: practical recommendations. Journal of Contemporary Trials 2015; doi: 10.1016/j.cct.2015.05.001.

Vera-Christina Mertens; Mariëlle Goossens; Jeanine Verbunt; Albere Köke; Rob Smeets. Effects of nurse-led motivational interviewing of patients with chronic musculoskeletal pain in preparation of rehabilitation treatment (PREPARE) on societal participation, attendance level, and cost-effectiveness: study protocol for a randomized controlled trial. Trials 2013; doi: 10.1186/1745-6215-14-90.

Anneke van Dijk-de Vries; Albine Moser, Vera-Christina Mertens, Jikke van der Linden, Trudy van der Weijden; Jacques T.M. van Eijk.

The ideal of biopsychosocial chronic care: How to make it real? A qualitative study among Dutch stakeholders. BMC Family Practice 2012; doi: 10.1186/1471-2296-13-14.

Vera-Christina Mertens; Hans Bosma; Danielle A.I. Groffen; Jacques Th.M. van Eijk. Good friends, high income or resilience? What matters most for elderly patients? The European Journal of Public Health 2011; doi: 10.1093/eurpub/ckr104.

\section{Submitted publications}

Vera-Christina Mertens, Mariëlle Goossens, Bjorn Winkens, Jeanine Verbunt, Rob Smeets. Effectiveness of a nurse-led Motivational-interviewing based pre-treatment in patients with non-specific chronic pain. (Submitted for publication).

Vera-Christina Mertens; Albine Moser; Jeanine Verbunt; Rob Smeets; Mariëlle Goossens. What does the CEO mean to you? Content validity of the Credibility and Expectancy Questionnaire (CEQ). (Submitted for publication). 
Vera-Christina Mertens, Jolanda van Haastregt, Mariëlle Goossens, Jeanine Verbunt, Rob Smeets.

Prepared for treatment? Process evaluation of a nurse-led pre-treatment in pain rehabilitation. (Submitted for publication).

\section{In preparation}

Vera-Christina Mertens, Ruud Pijls, Emile Curfs, Veronique Moulaert.

Impact of a Short-Message Service (SMS) responder system for out-out-hospital cardiac arrest: Survival, medical and operational costs, and survivors' quality of life.

\section{Book contribution}

Vera-Christina Mertens, Wilma Noteborn, Mariëlle Goossens (2014).

Book chapter 26: Motiverende gespreksvoering in de revalidatiesetting.

Handboek voor Revalidatiepsychologie. [Motivational interviewing in the rehabilitation setting. Handbook of rehabilitation psychology]. Uitgeverij Boom.

\section{Presentations}

Mertens, V.-C. and Jelsma, J.

How to measure motivational interviewing fidelity in randomized

controlled trials: practical recommendations. Poster presentation, 1st Dutch Congress of Motivational Interviewing, 25th September 2015, Zwolle, The Netherlands.

Mertens, V.-C.

Treatment Fidelity and Proficiency of a Nurse-led Motivational Interviewing (MI) based Pre-treatment in Patients with Non-specific Musculoskeletal Pain. Poster presentation, 9th Congress of the European Federation of IASP Chapters (EFIC) 2015, 2nd-5th September 2015, Vienna, Austria.

Mertens, V.-C.

Effectiveness and Moderators of the PREPARE RCT Using a Nurse-led Motivational Interviewing based Pre-Treatment in Patients with Non-specific Chronic Pain. Poster presentation, 9th Congress of the European Federation of IASP Chapters (EFIC) 2015, 2nd-5th September 2015, Vienna, Austria.

Mertens, V.-C.

What means the CEO to you? Content validity of the Credibility and Expectancy Questionnaire (CEQ) in a pain rehabilitation setting. Oral presentation, Dutch Congress of Rehabilitation Medicine (VRA), 6-7th November 2014, Rotterdam, The Netherlands. 
Mertens, V.-C.

Treatment fidelity a Motivational Interviewing (MI)-based pre-treatment in pain rehabilitation. Oral presentation, Pain Research Meeting, 4-5th September 2014, Vaeshartelt, The Netherlands.

Mertens, V.-C.

MI-fidelity of the Prepare trial. Oral presentation, 4th International Conference on Motivational Interviewing (ICMI), 16-18th June 2014, Amsterdam, The Netherlands.

Mertens, V.-C.

Effects of nurse-led Motivational Interviewing of patients with chronic musculoskeletal pain in preparation of rehabilitation treatment (PREPARE)- Concepts and mixed methods study design, 15-16th April 2013, Ecole des Hautes Etudes en Santé Publique (EHESP), Paris, France.

Mertens, V.-C., Goossens, M., Verbunt, J. Köke, A., Smeets, R.

(Cost-)effectiveness of PREPARE (PRe-Pain REhabilitation, design of a Motivational interviewing (MI)-based nurse-led intervention in patients with chronic musculoskeletal pain, a RCT. Oral presentation, 3rd International Conference on Motivational Interviewing (ICMI), 18-20th June 2012, Venice, Italy.

Mertens, V.-C.

(Cost-)effectiveness of PREPARE (PRE-Pain Rehabilitation), a Motivational interviewing (MI)-based nurse-led intervention in patients with chronic musculoskeletal pain, design of a RCT. Poster presentation, Dutch Congress of Rehabilitation Medicine (VRA) Annual Congress, 3-4th November 2011, Ermelo, The Netherlands.

Mertens, V.-C., Goossens, M., Verbunt, J. Köke, A., Smeets, R.

(Cost-) effectiveness of PREPARE (PRe-PAin REhabilition), a RCT of a motivational interviewing (MI)-based nurse-led intervention in patients with chronic musculoskeletal pain. Poster presentation, 7th Congress of the European Federation of IASP Chapters (EFIC) 2011, 21-24th September 2011, Hamburg, Germany.

Mertens V.-C., Bosma H., Groffen D.A.I., van Eijk J.Th.M.

Good friends, high income, or resilience: What matters for 'relatively successful functioning' in depressed elderly with a chronic disease? Oral presentation, Leiden International Medical Students' Conference (LIMSC), 16-20th March 2011, Leiden, The Netherlands. 


\section{Invited speaker}

Mertens, V.-C.

Motivational Interviewing in patients with chronic pain: Experiences, effectiveness, and practical recommendations. Dutch Congress of Motivational Interviewing, 25th September 2015, Zwolle, The Netherlands.

Mertens, V.-C.

Rehabilitation: a patient-physician dyad, Invited speaker mini-symposium, Dutch Congress of Rehabilitation Medicine (VRA), 3-4th November 2011, Ermelo, The Netherlands. 
Chronic musculoskeletal pain is a distressing health condition affecting a person's life and the social surrounding.

This thesis presents the results of the research project 'Pre-treatment Pain Rehabilitation' (Prepare). Therein, a pre-treatment was developed and evaluated by means of a randomized controlled trial and the role of expectancy was investigated.

Der Mensch sollte alle seine Werke zunächst einmal in seinem Herzen erwägen, bevor er sie ausführt.

Hildegard von Bingen (1098 - 1179) 